\title{
Search for Gluino and Squark Production in Multi-jets plus Missing Transverse Energy Final States at the Tevatron using the CDF Detector ${ }^{1}$
}

\author{
Xavier Portell i Bueso \\ Universitat Autònoma de Barcelona \\ Institut de Física d'Altes Energies \\ Edifici Cn Campus UAB \\ E-08193 Bellaterra (Barcelona) \\ e-mail: portell@ifae.es
}

January 2007

Supervised by:

Mario Martínez

IFAE

Edifici Cn Campus UAB

E-08193 Bellaterra (Barcelona)

${ }^{1}$ Ph.D. Dissertation 
A l'Ester, la meva dona, per la seva paciència i estima.

També per tots els qui m'heu recolzat $i$ animat en aquest temps a EEUU, en especial als meus pares $i$ germana.

Joy and amazement of the beauty and grandeur of this world of which man can just form a faint notion ...

\section{ALBERT EINSTEIN}

[Quotation on the Einstein Memorial in Washington] 


\section{Acknowledgments ${ }^{1}$}

This PhD Thesis constituted a considerable effort from a personal and academic point of view and it would not have been possible without the help and support from different people.

First of all, I want to thank my supervisor Mario Martínez for his energy and involvement. It has been a pleasure to work with a person with his great capability for overcoming the difficulties and for always aiming to perform a serious job. I also want to thank Matteo Cavalli-Sforza for his neverending passion for physics and for learning more about it. He was my master thesis supervisor but for different reasons he could not be my PhD supervisor. In any case, he always gave me courage and pieces of advice and I will always think of him as being part of it.

The IFAE postdocs at CDF have also helped me with the different difficulties that I needed to face during the development of the analysis. Regis was very important for the success of the DQM project and I want to thank his perseverance and his availability whenever I asked for help. I also want to thank Carlos for his inputs to the analysis and the support received from him in the bad moments. Finally, I want to mention Monica for being so kind and for transmitting, at any moment, the right amount of help, sympathy and energy to the students.

In addition, I want to thank all the CDF people that, in one way or another, contributed to the performance of this work or gave me support: the spokepersons, specially Jaco Konigsberg, the SUSY and Exotics conveners and all the people who gave me suggestions for the improvement of different parts of the analysis.

A very special thank deserve the IFAE students at CDF who have been my co-workers and have become very special friends. En primer lloc a l'Olga, per la seva amistat, la seva alegria i bon humor que han estat molt importants per a mi. També agrair-li les hores de xerrera per tal de desfogar-nos dels maldecaps, les hores de ball i les moltes bones estones compartides (gairebé totes!) en terres americanes. A l'Oriol agrair-li també la seva amistat, el seu tarannà sempre positiu, la seva capacitat encomiable de combinar treball i esbarjo, la seva inestimable ajuda en els problemes tècnics derivats de la nostra societat de la informació $\mathrm{i}$ tot el que he après dels seus "famosos scripts". D'altra banda, agrair a la Carolina la seva amistat, espontaneitat i simpatia. Hi ha poques persones que només amb un somriure et puguin alegrar el dia. Ella té aquesta virtut. El fet de poder compartir part d'aquesta experiència amb ella ha estat molt gratificant. Finally, I also want to thank Gianluca for his courage and determination.

Many other people at Fermilab have also been very important for me in these almost three years spent there. With them, we talked, laughed and did activities to recharghe the batteries for our everyday work. Aquí quiero mencionar a Oscar por su amistad, su sentido del humor, su ayuda siempre útil en el trabajo y por su comprensión y ánimos; a Kike por su característica forma de responder a las preguntas y por las aventuras vividas en Florida; a Jonatan por su "savoir-faire"; a Cristóbal por su interior; a Guillelmo por su trabajo; a Ernest por sus chistes y su bici; a Arán por ser tan afable y chistoso con todo el mundo; a Bárbara, Bruno, Miguel... y un largo etcétera. Con ellos las comidas, los “Talk, Mus \& Go”, el voleyball, el cine y muchos otros momentos han resultado inolvidables. At this point I should also mention people

\footnotetext{
${ }^{1}$ Aquest treball ha estat possible en part gràcies al suport del Departament d'Universitats, Recerca i Societat de la Informació, DURSI.
} 
like Evelyn, Melisa, Tuula, Kurt, Gian Piero, Eiko, Sunny... but the list would be too long and I would always forget somebody.

But although I spent most of the time at Fermilab, I want to thank all the support I received during my short periods at IFAE: seniors, postdocs, students... All of them have created a friendly atmosphere where I always felt as if I had never left. Here, I want to remark Enrique Fernández for the very warm welcome he gave me the first day I asked for the possibility of joining the IFAE group. I also want to mention the group of people with which we usually have good time taking lunch together. I am sure I will miss the nice discussions and jokes.

In addition, I want to thank all the friends who kept the contact with me during my stay abroad and had the patience to read my long e-mails. I want to stress that this contact has been very important for me in order to feel comfortable with living so far away from them.

Last (but not least) I want to thank my family for their great support during this time. Vull agrair la paciència dels avis, els correus i felicitacions dels tiets, les xerrades amb els meus cosins, l'alegria transmesa per sogres, cunyats i nebots i, en especial, el gran suport i empenta que m'han donat els meus pares i la meva germana. Part de la tesi és també gràcies a la seva manera de fer i la seva estima que sempre m'ha empès endavant. Entre altres coses, del meu pare agraeixo la seva sensibilitat envers les coses i la seva constància, de la meva mare la seva encomiable empenta i inacabable tendresa, de la meva germana el seu "carinyuuu" i les rialles encomanadisses. Tots ells han estat puntals on recolzar-me en el decurs d'aquests anys.

Finalment vull agrair a l'Ester, la meva dona, la immensa paciència que ha tingut amb mi $\mathrm{i}$ amb les circumstàncies que ens han portat a haver de conviure en la distància durant tant de temps. Tot plegat ha resultat una experiència enriquidora però també dura en la qual hem hagut de combinar la resignació del present amb la illlusió del futur. La seva estima, suport i constància han estat els elements bàsics, vitals, sense els quals no hagués arribat a realitzar el present treball. 


\section{Table of Contents}

$\begin{array}{llr}\text { List of Figures } & \text { v }\end{array}$

$\begin{array}{ll}\text { List of Tables } & \text { xv }\end{array}$

1 Introduction 1

2 Introduction to Supersymmetry 3

2.1 The Standard Model Framework . . . . . . . . . . . . . . . . . . . 3

2.1 .1 The $\mathrm{SM}$ content . . . . . . . . . . . . . . 3

2.1.2 Quantum Electrodynamics . . . . . . . . . . . . . . . . . 4

2.1 .3 Electroweak theory $\ldots \ldots \ldots \ldots \ldots \ldots$

2.1.4 The Higgs mechanism $\ldots \ldots \ldots \ldots$. . . . . . . . . . 7

2.1.5 Quantum Chromodynamics . . . . . . . . . . . . . . . 10

2.1.6 QCD description of the hadrons . . . . . . . . . . . . . . 11

2.1.7 PDF parameterisations . . . . . . . . . . . . . 13

2.1 .8 PDF uncertainties . . . . . . . . . . . . . . . . . . 13

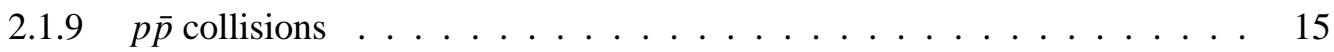

2.1.10 Initial- and Final-State Radiation (ISR/FSR) . . . . . . . . . . . . 15

2.1.11 Hadronisation . . . . . . . . . . . . . . . . . . . . . 16

2.2 The limitations of the Standard Model _ . . . . . . . . . . . . . . . . 17

2.2 .1 The hierarchy problem . . . . . . . . . . . . . . . . 19

2.3 The Supersymmetric extension of the Standard Model . . . . . . . . . . . . . 20

2.3 .1 Supersymmetry . . . . . . . . . . . . . . . . . 20 
2.3.2 Supersymmetry and the hierarchy problem . . . . . . . . . . . 20

2.3.3 Other benefits from the introduction of SUSY . . . . . . . . . . . . 21

2.3.4 The Minimal Supersymmetric Standard Model _ . . . . . . . . . . . . 22

2.3.4.1 MSSM lagrangian and R-parity . . . . . . . . . . . . . 26

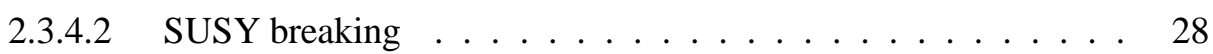

2.3.5 Constraining the MSSM: mSUGRA model . . . . . . . . . . . . . 29

2.4 Squarks and Gluinos $\ldots \ldots \ldots \ldots$. . . . . . . . . . . . . . 32

2.5 Squark and Gluino Production Processes . . . . . . . . . . . . . . 33

2.6 Squark and Gluino Decays _. . . . . . . . . . . . . . . . . . . . 34

2.7 Experimental constraints: Run I limits . . . . . . . . . . . . . . . . . . . 34

3 Experimental Setup $\quad 39$

3.1 The Tevatron in Run II . . . . . . . . . . . . . . . . . . . . . . . . . . . . . 39

3.2 The Collider Detector at Fermilab _. . . . . . . . . . . . . . . . 42

3.2.1 General Characteristics . . . . . . . . . . . . . . . 42

3.2.2 Standard Definitions at CDF . . . . . . . . . . . . . . 43

3.2 .3 The Silicon detector $\ldots \ldots$. . . . . . . . . . . . . . . . . 43

3.2.4 The Central Outer Tracker . . . . . . . . . . . . . . . . . . . . . . . 44

3.2 .5 The Time of Flight $\ldots \ldots \ldots$. . . . . . . . . . . . . . 46

3.2.6 The Calorimeters . . . . . . . . . . . . . . . . . . . . 46

3.2.7 The Muon System _ . . . . . . . . . . . . . . . . . . 50 50

3.2 .8 The Trigger System . . . . . . . . . . . . . . . . . . . . 50

3.2.9 CLC and luminosity measurement . . . . . . . . . . . . . 52

3.2.10 Data Quality Monitoring and Validation . . . . . . . . . . . . 54

4 Analysis Method

4.1 Data Pre-selection . . . . . . . . . . . . . . . . . 55

$4.1 .1 \quad$ Trigger Path . . . . . . . . . . . . . . . . 55

$4.1 .2 \quad$ Pre-selection Cuts $\ldots \ldots \ldots \ldots \ldots$

4.2 Signal Generation and Normalisation . . . . . . . . . . . . . . . . . 58 
4.3 Background Processes . . . . . . . . . . . . . . . . . . . 60

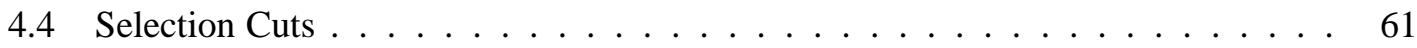

4.4.1 Multijet Background (QCD) Rejection . . . . . . . . . . . . . . . . . . 61

4.4 .2 Electron Rejection $\ldots \ldots \ldots$. . . . . . . . . . . 63

4.4 .3 Muon Rejection . . . . . . . . . . . . . . . . . . . . 63

4.4.4 Further Rejection: Signal vs Background Optimisation . . . . . . . . . . 65

4.5 Signal Studies . . . . . . . . . . . . . . . . . . . . . . . . . . . 69

4.6 Systematic Uncertainties . . . . . . . . . . . . . . . . . . . . . . . . . 84

4.6.1 Renormalisation Scale . . . . . . . . . . . . . . . . . . . 84

4.6 .2 PDF uncertainty $\ldots \ldots \ldots \ldots \ldots \ldots$

4.7 Results . . . . . . . . . . . . . . . . . . . . . 93

4.7.1 Highest energetic event . . . . . . . . . . . . . . . . 102

4.8 Limit Calculation . . . . . . . . . . . . . . . . . . . . 102

5 Conclusions and Future Prospects 109

$\begin{array}{ll}\text { Bibliography } & 111\end{array}$

$\begin{array}{lll}\text { A Data Quality Monitoring } & 117\end{array}$

A.1 Introduction . . . . . . . . . . . . . . . . . . . . 117

A.2 The offline DQM system . . . . . . . . . . . . . . . . . 117

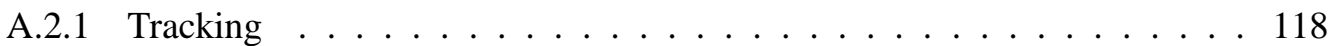

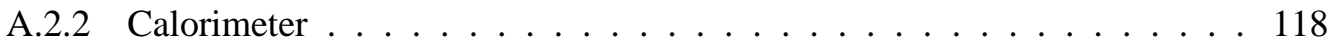

A.2.3 Muons . . . . . . . . . . . . . . . . . 120

$\begin{array}{lll}\text { B } & \text { Trigger Studies } & 121\end{array}$

C Beam Backgrounds and Cosmic Rays 125

C.1 Cuts to remove beam-related backgrounds . . . . . . . . . . . . . . . . . 127

C.1.1 ECHF and EEMF variables $\ldots \ldots \ldots \ldots$

C.2 Study of the beam losses in the plug . . . . . . . . . . . . . . . 130 
D Track Validation

133

E PYTHIA vs ISAJET

135

F QCD Studies

137

G Data vs MC Studies

149 


\section{List of Figures}

2.1 CTEQ6 parton distribution functions at $Q=2 \mathrm{GeV}$ and $Q=100 \mathrm{GeV} \ldots \ldots$. . . 14

2.2 Uncertainty bands for the $u$-quark (left) or gluon (right) distribution functions at $Q^{2}=10 \mathrm{GeV}^{2}$. The other lines are from different type of parameterisations not discussed here. . . . . . . . . . . . . . . . . . . 14

2.3 An example of the different processes occurring at a $p \bar{p}$ collision. . . . . . . . . 16

2.4 A representation of the string (left) and cluster (right) fragmentation models. . . . 18

2.5 One-loop quantum corrections to $m_{h_{S M}}^{2} \ldots \ldots \ldots \ldots \ldots \ldots \ldots$

2.6 $M_{W}$ as a function of $m_{t}$ as predicted by the SM in red (medium-shaded) and blue (dark-shaded) bands and with the MSSM prediction in green (light-shaded) and blue (dark-shaded) bands. The perspectives for the present and future generation colliders, are also stated. . . . . . . . . . . . . . . . .

2.7 The running of the sparticle masses from the GUT scale to the electroweak scale, for a sample set of input parameters. The bold lines represent the three soft gaugino masses $m_{\tilde{g}}, M_{2}$ (labeled $\tilde{W}$ ) and $M_{1}$ (labeled $\tilde{B}$ ). The light solid lines are the squark $\left(\tilde{q}_{L}, \tilde{q}_{R}, \tilde{t}_{L}, \tilde{t}_{R}\right)$ and the slepton $\left(\tilde{l}_{L}, \tilde{l}_{R}\right)$ soft masses. Finally the dashed lines represent the soft Higgs boson masses labeled by $H_{d}$ and $H_{u}$. Figure taken from [44]. 31

2.8 Feynman diagrams for the production of squarks and gluinos in lowest order. The diagrams in (c) and the last diagram in (d) are result of the Majorana nature of gluinos. Some of the above diagrams contribute only for specific flavours and chiralities of the squarks $[48] . \ldots \ldots \ldots \ldots \ldots$

2.9 The relative yields of squarks and gluinos in the final states at the Tevatron. The mass ratio $m_{\tilde{q}} / m_{\tilde{g}}$ is chosen to be (a) 0.8 and (b) 1.6. Also shown are the leading parton contributions for (c) $\tilde{q} \overline{\tilde{q}}$ and (d) $\tilde{g} \tilde{g}$ final states. . . . . . . . . . . . . 36

$2.10 m_{\tilde{q}}-m_{\tilde{g}}$ exclusion plot at the $95 \%$ C.L assuming R-parity conservation for $\tan \beta=$ 3. Results form the previous searches are also shown. . . . . . . . . . . 37 
3.1 Layout of the Fermilab accelerator complex. . . . . . . . . . . . . . . . . . 40

3.2 Isometric view of the CDF Run II detector. . . . . . . . . . . . . . . . . . . . . 42

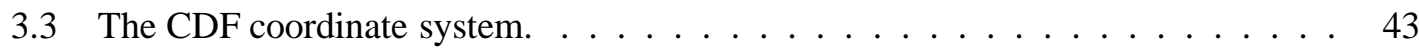

3.4 Longitudinal view of the CDF Run II tracking system. . . . . . . . . . . . . 44

3.5 Wire layout in a COT supercell of SL2 . . . . . . . . . . . . . . . 45

3.6 Schematic $r-z$ view of the $\eta$ coverage of the inner and outer tracker (SVX II, ISL and COT). . . . . . . . . . . . . . . . . . . . . . 46

3.7 Elevation view of one half of the CDF detector displaying the different components of the $\mathrm{CDF}$ calorimeter. . . . . . . . . . . . . . . . . . 47

3.8 Central hadronic calorimeter module. The light scheme is sketched. . . . . . . . 49

3.9 The CDF Run II Trigger and Data Acquisition System. . . . . . . . . . . . . . 51

3.10 Block diagram of the CDF Run II Trigger System. . . . . . . . . . . . . . . . 52

3.11 Event Builder and L3 filtering. Data from the front end crates pass through ATM switches to the converter nodes. Here, the events are assembled and passed to the processor nodes. The accepted events are passed to output nodes which send them to the Consumer Server and Data Logging systems (CS/DL) . . . . . . . . . 53

3.12 Schematic view of the luminosity monitor inside a quadrant of CDF. It is located

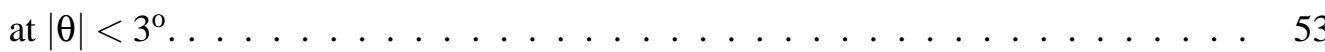

4.1 Cross section vs run number for the MET35 trigger. The plot includes a number of cuts to pre-select the data. . . . . . . . . . . . . . . . 56

$4.2 \quad E_{T}$ spectrum before and after applying the pre-selection (or basic) cuts . . . . . 58

4.3 mSUGRA points generated with PYTHIA. The y-axis is the average mass of the 8 squarks of the first two generations. The $\mathrm{x}$-axis corresponds to the mass of the gluino. 15,000 events were generated for each point. The plot is divided into the three zones obtained from the optimisation study. . . . . . . . . . . . . . . .

4.4 mSUGRA points generated with PYTHIA in the context of the limits found by previous analyses. The y-axis is the average mass of the 8 squarks of the first two generations and the $\mathrm{x}$-axis corresponds to the mass of the gluino. . . . . . . . 60

4.5 $\Delta \phi\left(\mathbb{E}_{T}\right.$, jets $)$ distributions for the three leading jets of the QCD multijet sample. The peak at zero comes from events in which one of the jets is mismeasured resulting in a $\mathbb{E}_{T}$ aligned with the jet. The arrow indicates the value of the cut. . . 
4.6 $\Delta \phi\left(\mathbb{E}_{T}\right.$, jets $)$ distributions for the three leading jets of the representative mSUGRA point: s35 (top), s56 (middle), s80 (bottom). The $\mathbb{E}_{T}$ in signal events points in a direction away from the jets. The arrow indicates the value of the cut. . . . . . .

4.7 EMF distributions for the three leading jets of the representative mSUGRA points: s35 (top), s56 (middle), s80 (bottom). The arrow indicates the value of the cut. . .

4.8 EMF distribution for the leading jets of the $\mathrm{W} \rightarrow \mathrm{ev}$ sample. A large fraction of the jets have an EMF close to one. These jets are most likely electrons. The arrow indicates the value of the cut. . . . . . . . . . . . . . . . . .

4.9 The left column shows the distribution of the invariant mass of the two highest isolated tracks. The right column shows the distribution of the azimuthal angle between the $\mathbb{E}_{T}$ and the highest isolated track. Plots are shown for s35 (top), s56 (middle), and s80 (bottom). The arrows show where the different cuts are placed.

4.10 Invariant mass distribution of the two highest isolated tracks in the event. The plot on the left corresponds to $Z / \gamma^{*} \rightarrow \mu \mu$, while the plot on the right corresponds to $W \rightarrow \mu \nu$. This cut does little to reduce the $W \rightarrow \mu \nu$ background since not many of the events have two or more isolated tracks. However, it rejects most of the $Z / \gamma^{*} \rightarrow \mu \mu$ background. The arrows show were the invariant mass window cut is

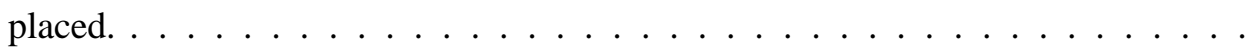

$4.11 \Delta \phi\left(\right.$ track, $\left.\mathbb{E}_{\mathrm{T}}\right)$ distribution for $Z / \gamma^{*} \rightarrow \mu \mu$ events (left) and $W \rightarrow \mu \nu$ events (right). This cut rejects events in which the direction of the $\mathbb{E}_{T}$ is aligned with that of an isolated track. A significant fraction of the backgrounds is rejected by the cut placed where the arrow indicates. . . . . . . . . . . . . 66

$4.12 \mathrm{~S} / \sqrt{\mathrm{B}}$ distribution for $\mathrm{H}_{\mathrm{T}}$ of the mSUGRA points along the diagonal. . . . . . . 68

$4.13 \mathrm{~S} / \sqrt{\mathrm{B}}$ distributions for $\mathrm{H}_{\mathrm{T}}$ of the mSUGRA points along the row. . . . . . . . . . 69

$4.14 \mathrm{H}_{\mathrm{T}}$ distributions for all the points in the diagonal $\left(\mathrm{M}_{\tilde{\mathrm{q}}} \approx \mathrm{M}_{\tilde{\mathrm{g}}}\right)$. The arrow shows where the cut is placed for each different zone. The first two plots correspond to zone A (points s23, and s35), the next four plots correspond to zone B (points s46, s56, and s65), and the last three plots correspond to zone C (points s73, s80, s86, and s91) . . . . . . . . . . . . . . . . . . .

4.15 $\mathrm{H}_{\mathrm{T}}$ distributions for all the points in the chosen row (gluino mass increases as the point number increases). The arrow shows where the cut is placed for each different zone. The first two plots correspond to zone A (points s31, and s42), the next four histograms correspond to zone B (points s52, s61, and s69), and the last three plots correspond to zone $\mathrm{C}$ (points $\mathrm{s} 76, \mathrm{~s} 82$, s87, and s91). . . . . . . 71

$4.16 \mathrm{~S} / \sqrt{\mathrm{B}}$ distribution for $\mathbb{E}_{T}$ of the mSUGRA points along the diagonal. . . . . . . 72 
$4.17 \mathrm{~S} / \sqrt{\mathrm{B}}$ distributions for $\mathbb{E}_{T}$ of the mSUGRA points along the row. . . . . . 73

4.18 $\mathbb{E}_{T}$ distributions for all the points in the diagonal $\left(\mathrm{M}_{\tilde{\mathrm{q}}} \approx \mathrm{M}_{\tilde{\mathrm{g}}}\right)$. The arrow shows where the cut is placed for each different zone. The first two histograms correspond to zone A (points s23, and s35), the next four plots correspond to zone B (points s46, s56, and s65), and the last three plots correspond to zone $\mathrm{C}$ (points s73, s80, s86, and s91). . . . . . . . . . . . . . . . . .

4.19 $\mathbb{E}_{T}$ distributions for all the points in the chosen row (gluino mass increases as the point number increases). The arrow shows where the cut is placed for each different zone. The first two plots correspond to zone A (points s31, and s42), the next four plots correspond to zone B (points s52, s61, and s69), and the last three plots correspond to zone $\mathrm{C}$ (points s76, s82, s87, and s91) . . . . . . . . . . .

4.20 S/ $\sqrt{\mathrm{B}}$ distribution for $\mathrm{E}_{\mathrm{T}}^{\text {jet } 1}$ of the $\mathrm{mSUGRA}$ points along the diagonal $\left(\mathrm{M}_{\tilde{\mathrm{q}}} \approx \mathrm{M}_{\tilde{\mathrm{g}}}\right)$. After applying the $\mathrm{H}_{\mathrm{T}}$ and $\mathbb{E}_{T}$, the signal and background distributions have the same shape. Therefore, the $S / \sqrt{B}$ becomes flat until it starts to drop. . . . . . . .

$4.21 \mathrm{E}_{\mathrm{T}}^{\mathrm{jet} 1}$ distributions for points $\mathrm{s} 35$, $\mathrm{s} 56$, and $\mathrm{s} 80$ on the left, and points $\mathrm{s} 42$, $\mathrm{s} 61$, and s82 on the right (each pair of histograms corresponds to points belonging to the same column (same gluino mass) in Fig. 4.3). The cut is chosen to remove the low-end tails of the signal distributions. . . . . . . . . . . . . . .

$4.22 \mathrm{E}_{\mathrm{T}}^{\mathrm{jet} 2}$ distributions for points $\mathrm{s} 35$, $\mathrm{s} 56$, and $\mathrm{s} 80$ on the left, and points $\mathrm{s} 42$, $\mathrm{s} 61$, and s 82 on the right (each pair of histograms corresponds to points belonging to the same column (same gluino mass) in Fig. 4.3). The cut is chosen to remove the low-end tails of the signal distributions.

$4.23 \mathrm{E}_{\mathrm{T}}^{\mathrm{jet} 3}$ distributions for points $\mathrm{s} 35, \mathrm{~s} 56$, and $\mathrm{s} 80$ on the left, and points $\mathrm{s} 42$, s61, and $\mathrm{s} 82$ on the right (each pair of histograms corresponds to points belonging to the same column (same gluino mass) in Fig. 4.3). We do not apply a cut to the energy of the third jet except for the pre-selection cut of $25 \mathrm{GeV}$. . . . . . . .

4.24 Different relative contributions of the signal production processes before the cuts (top-left), after the analysis cuts (top-right), the signal efficiencies (bottom-left) and significances (bottom-right) along a column $\left(M_{\tilde{g}} \approx 185 \mathrm{GeV} / \mathrm{c}^{2}\right) . \ldots \ldots$. . .

4.25 Different relative contributions of the signal production processes before the cuts (top-left), after the analysis cuts (top-right), the signal efficiencies (bottom-left) and significances (bottom-right) along the diagonal $\left(M_{\tilde{g}} \approx M_{\tilde{q}}\right) \ldots \ldots \ldots$. . . .

4.26 Different relative contributions of the signal production processes before the cuts (top-left), after the analysis cuts (top-right), the signal efficiencies (bottom-left) and significances (bottom-right) along a row $\left(M_{\tilde{q}} \approx 355 \mathrm{GeV} / \mathrm{c}^{2}\right) \ldots \ldots \ldots$. . . 
$4.27 \sigma_{g g}, \sigma_{s g}, \sigma_{s s}$, and $\sigma_{s b}$ distributions for the 40 different PDFs (points) and the nominal PDF (line) for mSUGRA point 35. Each eigenvalue has its plus and minus deviation represented in successive points. The largest deviation corresponds to eigenvalue 15 which is associated with the high-x gluon content. The $\sigma_{s b}$ plot does not show such deviation as it is dominated by $\mathrm{q} \overline{\mathrm{q}}$ annihilation. . . . . . . . . .

$4.28 \sigma_{g g}, \sigma_{s g}, \sigma_{s s}$, and $\sigma_{s b}$ distributions for the 40 different PDFs (points) and the nominal PDF (line) for mSUGRA point 56. Each eigenvalue has its plus and minus deviation represented in successive points. The largest deviation corresponds to eigenvalue 15 which is associated with the high-x gluon content. The $\sigma_{s b}$ plot does not show such deviation as it is dominated by $\mathrm{q} \overline{\mathrm{q}}$ annihilation. . . . . . . . .

$4.29 \sigma_{g g}, \sigma_{s g}, \sigma_{s s}$, and $\sigma_{s b}$ distributions for the 40 different PDFs (points) and the nominal PDF (line) for mSUGRA point 80. Each eigenvalue has its plus and minus deviation represented in successive points. The largest deviation corresponds to eigenvalue 15 which is associated with the high-x gluon content. The $\sigma_{s b}$ plot does not show such deviation as it is dominated by $\mathrm{q} \overline{\mathrm{q}}$ annihilation. . . . . . . . .

$4.30 k$-factors for the 40 different PDFs (points) and the nominal PDF (line) for $W+2$

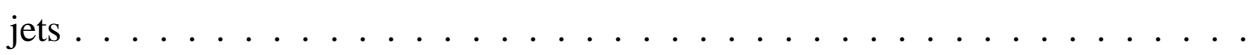

$4.31 k$-factors for the 40 different PDFs (points) and the nominal PDF (line) for $Z+2$

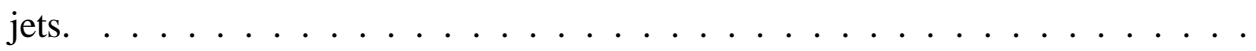

$4.32 k$-factors for the 40 different PDFs (points) and the nominal PDF (line) for $W W$

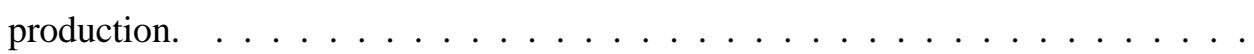

4.33 Systematic uncertainties relative to the signal efficiencies for the three representative $\mathrm{mSUGRA}$ points $\mathrm{s} 35, \mathrm{~s} 56$ and $\mathrm{s} 80 \ldots \ldots \ldots \ldots \ldots$

4.34 Individual backgrounds relative contributions to the total one after all the cuts have been applied for Zone A (top), Zone B (middle), Zone C (bottom). . . . . .

4.35 $\mathrm{H}_{\mathrm{T}}$ distribution for the expected number of mSUGRA signal events, and background events in region A. The points correspond to the data events which pass the cuts for the region. Distributions have passed all the cuts except the $\mathrm{H}_{\mathrm{T}}$ one. .

4.36 $\mathbb{E}_{T}$ distribution for the expected number of mSUGRA signal events, and background events in region A. The points correspond to the data events which pass the cuts for the region. Distributions have passed all the cuts except the $\mathbb{E}_{T}$ one.

4.37 $\mathrm{H}_{\mathrm{T}}$ distribution for the expected number of mSUGRA signal events, and background events in region B. The points correspond to the data events which pass the cuts for the region. Distributions have passed all the cuts except the $\mathrm{H}_{\mathrm{T}}$ one. 
$4.38 \mathscr{E}_{T}$ distribution for the expected number of mSUGRA signal events, and background events in region $\mathrm{B}$. The points correspond to the data events which pass the cuts for the region. Distributions have passed all the cuts except the $\mathbb{E}_{T}$ one. . 100

4.39 $\mathrm{H}_{\mathrm{T}}$ distribution for the expected number of mSUGRA signal events, and background events in region $\mathrm{C}$. The points correspond to the data events which pass the cuts for the region. Distributions have passed all the cuts except the $\mathrm{H}_{\mathrm{T}}$ one. . 101

4.40 $\mathbb{E}_{T}$ distribution for the expected number of mSUGRA signal events, and background events in region $\mathrm{C}$. The points correspond to the data events which pass the cuts for the region. Distributions have passed all the cuts except the $\mathbb{E}_{T}$ one. . 101

4.41 CDF central tracking chamber and calorimeter ( $r-\phi$ view) (top) and Calorimeter "Lego" plot (bottom) for the event with the highest $\mathbb{E}_{T}$ and $\mathrm{H}_{\mathrm{T}}$. This event has three jets above $25 \mathrm{GeV}\left(E_{T}^{1}=236 \mathrm{GeV}, E_{T}^{2}=150 \mathrm{GeV}, E_{T}^{3}=84 \mathrm{GeV}\right)$ with $\mathrm{H}_{\mathrm{T}}=470 \mathrm{GeV}$ and $\mathbb{E}_{T}=196 \mathrm{GeV} \ldots \ldots \ldots \ldots$

4.42 Cross section as a function of squark mass for two $\mathrm{M}_{\tilde{\mathrm{g}}}$ values (columns). The observed and expected limits at 95\% C.L. are also shown. The yellow band shows the total effect of the PDFs and the renormalisation scale uncertainties. . . . . . . 105

4.43 Cross section as a function of gluino/squark masses in the case of $\mathrm{M}_{\tilde{\mathrm{q}}} \approx \mathrm{M}_{\tilde{\mathrm{g}}}$ (diagonal). The observed and expected limits at 95\% C.L. are also shown. The yellow band shows the total effect of the PDFs and the renormalisation scale uncertainties. 106

$4.44 \mathrm{M}_{\tilde{\mathrm{q}}}-\mathrm{M}_{\tilde{\mathrm{g}}}$ exclusion plot at the $95 \%$ C.L in the framework of mSUGRA assuming R-parity conservation. The pink region was excluded by the UA1 experiment [52]. The yellow region was excluded by the UA2 experiment [53]. The blue region was excluded by the CDF and D $\varnothing$ experiments after Run I [51]. The brown region was excluded by the LEP experiment [81]. In the gray region, the squark mass is lower than the mass of the lightest neutralino. In the black hashed region there is no mSUGRA solution. The green region shows the area excluded by the present analysis with $371 \mathrm{pb}^{-1}$ of CDF Run II data. . . . . . . . . . . 107

A.1 Hit residual distributions for SL1 (left) and SL8 (right) for a particular run. . . . 118

A.2 Occupancy of electromagnetic (left) and hadronic (right) towers for low (up) and high (down) energy thresholds for a particular run. . . . . . . . . . . . 119

B.1 L1 trigger efficiency curve with respect to the offline $\mathbb{E}_{T}$ using high $p_{T}$ muon sample (left) and Jet20 (right). . . . . . . . . . . . . . . . . . . . 122 
B.2 L2 trigger efficiency curve with respect to the transverse energy of the second jet (left) or the third jet (right). Both distributions are implemented using the pre-scaled missing transverse energy trigger. . . . . . . . . . . . . . . . .

B.3 L3 trigger efficiency curve with respect to the offline $\mathbb{E}_{T}$ using high $p_{T}$ muon

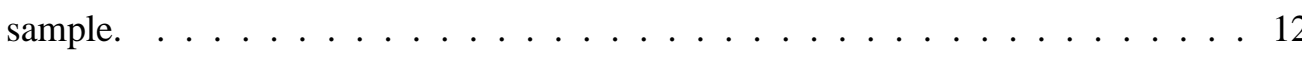

C.1 $\mathscr{E}_{T} \phi$ distribution without applying any cut. The peaks at different regions can be explained from the presence of other type of backgrounds not coming from the

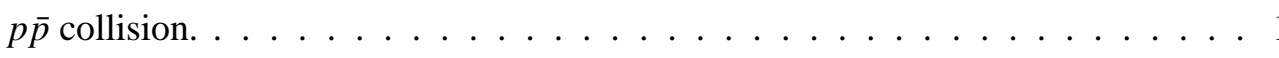

C.2 Beam halo particles trajectory through the CDF calorimetry. . . . . . . . . 126

C.3 Example of beam halo energy deposition. . . . . . . . . . . . . . . . . 126

C.4 $\mathrm{X}$ and $\mathrm{Y}$ components of the transverse energy deposited in the closest towers to the beam line for the east plug. . . . . . . . . . . . . . . . . . 127

C.5 $\mathbb{E}_{T}$ spectrum in $\phi$ of a subset of the data after applying the $\mathbb{E}_{T}>45 \mathrm{GeV}$ cut (left) or the vertex and two jets requirements (right). . . . . . . . . . . 128

C.6 $\mathscr{E}_{T}$ spectrum in $\phi$ of a subset of the data after applying the $\mathbb{E}_{T}>45 \mathrm{GeV}$ cut and the requirements of one vertex $\left(V_{Z}<60 \mathrm{~cm}\right)$ and at least two jets. . . . . . 128

C.7 EEMF for data and all the SM backgrounds (MC simulated). The plot includes the pre-selection cuts except the cuts on EEMF and ECHF.

C.8 ECHF for data and MC. The plot includes the pre-selection cuts except the cuts for EEMF and ECHF. . . . . . . . . . . . . . . . . . . . 130

C.9 $\mathbb{E}_{T}$ spectrum in $\phi$, with the characteristic peaks already presented, with the whole calorimeter (left), with only considering $|\eta|<1.93$ towers (middle) and without

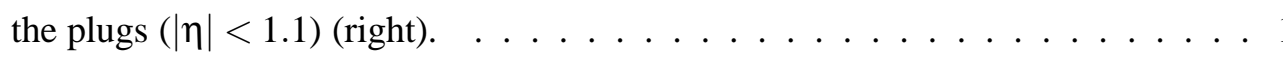

C.10 Mean transverse energy deposited in the east (left) or west (right) plugs in different $\phi$ regions. The $\phi$ regions cover $60^{\circ}$ and are numbered from 1 to 6 , starting

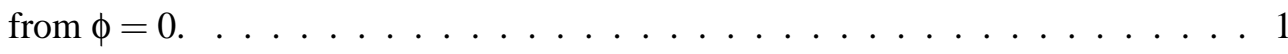

D.1 Invariant mass of the two highest isolated tracks for bhmu0d data and $Z \rightarrow \mu \mu$ Monte Carlo. . . . . . . . . . . . . . . . . . . . . . . . . . . . 134

D.2 Number of isolated tracks just after the pre-selection cuts using the emet0d data sample. . . . . . . . . . . . . . . . . . . . . . 13

E.1 Mass differences between PYTHIA and ISAJET for gluino (left) and squarks (right)135 
E.2 Difference on $\hat{s}$ between ISAJET and PYTHIA (left). Difference on the transverse energy of the leading jet at the hadron level (right). . . . . . . . . . . . 136

F.1 Distribution of number of jets in the QCD control region. . . . . . . . . . . 138

F.2 Distribution of the $E_{T}^{\text {jet1 }}$ inside the QCD control region. . . . . . . . . . . . . 139

F.3 Distribution of the $E_{T}^{\text {jet2 }}$ inside the QCD control region. . . . . . . . . . . . . 139

F.4 Distribution of the $E_{T}^{\text {jet3 }}$ inside the QCD control region. . . . . . . . . . . . . 140

F.5 Distribution of $\mathbb{E}_{T}$ inside the QCD control region. . . . . . . . . . . . . . 140

F.6 Ratio between data and MC for the $\mathbb{E}_{T}$ distribution inside the QCD control region. 141

F.7 Definition of zones in $\mathbb{E}_{T}$ vs $H_{T}$ plane inside the QCD control region. . . . . . 141

F.8 Distribution of $\mathbb{E}_{T}$ inside the QCD control region (zone 1). . . . . . . . . . 142

F.9 Distribution of $\mathbb{E}_{T}$ inside the QCD control region (zone 2). . . . . . . . . . . 142

F.10 Distribution of $\mathbb{E}_{T}$ inside the QCD control region (zone 3). . . . . . . . . . 143

F.11 Distribution of $\mathbb{E}_{T}$ inside the QCD control region (zone 4). . . . . . . . . . 143

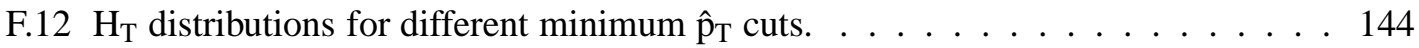

F.13 Ratio between the $\mathrm{H}_{\mathrm{T}}$ distributions of data and $\mathrm{MC}$ for three different $\hat{\mathrm{p}}_{\mathrm{T}}$ cuts: 60,95 , and $130 \mathrm{GeV}$ respectively. The black line corresponds to the fit of the distribution. . . . . . . . . . . . . . . . . . . . . 145

F.14 $\mathrm{E}_{\mathrm{T}}$ distribution of the second leading jet for different minimum $\hat{\mathrm{p}}_{\mathrm{T}}$ cuts. $\ldots \ldots$

F.15 Ratio between the second leading jet's $\mathrm{E}_{\mathrm{T}}$ distributions of data and $\mathrm{MC}$ for three different $\hat{p}_{\mathrm{T}}$ cuts: 60,95 , and $130 \mathrm{GeV}$, respectively. The black line corresponds to the fit of the distribution. . . . . . . . . . . . . . . . 146

F.16 $\chi^{2} /$ d.o.f results from fitting the $\mathrm{H}_{\mathrm{T}}$ distributions to the data. The results suggest a minimum $\hat{p}_{\mathrm{T}}$ cut around $95 \mathrm{GeV} \ldots \ldots \ldots \ldots 147$

F.17 $\chi^{2} /$ d.o.f results from fitting the $\mathrm{E}_{\mathrm{T}}^{2}$ distributions to the data. The results above suggest a minimum $\hat{p}_{\mathrm{T}}$ cut around $100 \mathrm{GeV} \ldots \ldots \ldots \ldots$

G.1 Azimuthal distance between $\mathbb{E}_{T}$ and $\mathrm{E}_{\mathrm{T}}$ of the three leading jets for data and MC after pre-selection, electron and muon removal cuts have been applied. . . . . . . 151

G.2 Detector $\eta$ of the three leading jets for data and MC after pre-selection, electron and muon removal cuts have been applied. . . . . . . . . . . . . . . . 152 
G.3 $\mathrm{E}_{\mathrm{T}}$ of the three leading jets for data and $\mathrm{MC}$ after pre-selection, electron and muon removal cuts have been applied. There are 10551 data events and $9.87_{-2.33}^{+1.35} \cdot 10^{3}$ Monte Carlo events in the plots. . . . . . . . . . . . . . . . . . 153

G.4 Azimuthal distance between $\mathbb{E}_{T}$ and $\mathrm{E}_{\mathrm{T}}$ of the three leading jets for data and $\mathrm{MC}$ after the $\Delta \phi\left(\mathbb{E}_{T}\right.$, jets $)>0.7$ cuts. Pre-selection, electron and muon removals cuts have also been applied. There are 1204 data events and $1.29_{-0.17}^{+0.14} \cdot 10^{3} \mathrm{MC}$ events in the plots.

G.5 Detector $\eta$ of the three leading jets for data and $\mathrm{MC}$ after the $\Delta \phi\left(\mathbb{E}_{T}\right.$, jets $)>0.7$ cuts. Pre-selection, electron and muon removals cuts have also been applied. . . . 155

G.6 $\mathrm{E}_{\mathrm{T}}$ of the three leading jets for data and $\mathrm{MC}$ after the $\Delta \phi\left(\mathbb{E}_{T}\right.$,jets $)>0.7$ cuts. Pre-selection, electron and muon removals cuts have also been applied. . . . . . . 156

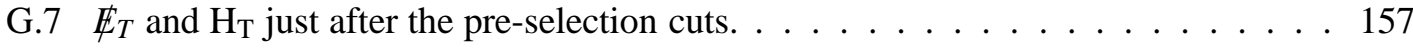

G.8 $\mathbb{E}_{T}$ and $\mathrm{H}_{\mathrm{T}}$ after all cuts in the analysis except the $\mathbb{E}_{T}$ and $\mathrm{H}_{\mathrm{T}}$ cuts respectively, but reversing the $\Delta \phi\left(\mathbb{E}_{T}\right.$,jets $)>0.7$ cut by asking at least one of the three jets to fail the condition. All three zones are shown. . . . . . . . . . . . . . 158

G.9 $\mathbb{E}_{T}$ and $\mathrm{H}_{\mathrm{T}}$ after all cuts in the analysis except the $\mathbb{E}_{T}$ and $\mathrm{H}_{\mathrm{T}}$ cuts respectively, but reversing the $\Delta \phi\left(\mathbb{E}_{T}\right.$, jets $)>0.7$ cut by asking at least one of the three jets to fail the condition. All three zones are shown. . . . . . . . . . . . . . . . . 159

G.10 $\mathscr{E}_{T}$ and $\mathrm{H}_{\mathrm{T}}$ after all cuts in the analysis except the $\mathbb{E}_{T}$ and $\mathrm{H}_{\mathrm{T}}$ cuts respectively, but reversing the EMF $>0.9$ cuts by asking at least one of the three jets to fail the condition. All three zones are shown. . . . . . . . . . . . . . . . . . . . . . 160

G.11 $\mathscr{E}_{T}$ and $\mathrm{H}_{\mathrm{T}}$ after all cuts in the analysis except the $\mathscr{E}_{T}$ and $\mathrm{H}_{\mathrm{T}}$ cuts respectively, but enhancing the muon contribution by requiring at least one isolated track. All 


\section{List of Tables}

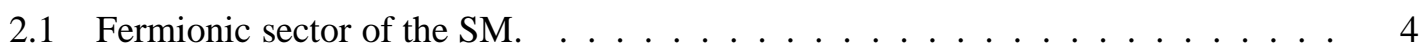

2.2 Standard Model gauge bosons and the corresponding interactions. . . . . . . . . 4

2.3 Superfields and particle content of the MSSM. Symbols for each of the chiral supermultiplets as a whole are indicated in the second column. . . . . . . . . . . 24

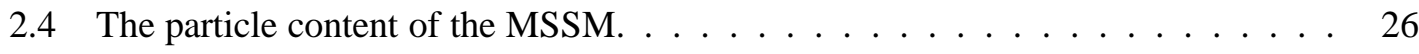

3.1 CDF II calorimetry summary. . . . . . . . . . . . . . . . . . . . . . . . . 49

4.1 Summary of the MET35 trigger logic used in collecting the data. . . . . . . . . . 55

4.2 List of the SM background processes considered for this analysis and the normalisation to $\mathrm{NLO}(k$-factor $) . \ldots \ldots \ldots 61$

4.3 Cut thresholds for the three different regions in which the signal plane is divided. 69

4.4 Efficiencies for mSUGRA events in regions A, B, and C. The points shown are representative for each region. . . . . . . . . . . . . . 80

4.5 Systematic uncertainties on the selection efficiency for some representative mSUGRA

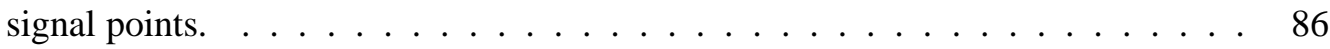

4.6 Systematic uncertainties on the background expected number of events (in number of events and with respect to the total background expectations) for the different regions considered in the analysis. . . . . . . . . . . . . 86

4.7 Systematic uncertainty on the background expected number of events due to the uncertainty on the renormalisation scale. . . . . . . . . . . . 87

4.8 Systematic uncertainty on the background expected number of events due to the uncertainty on the PDFs. . . . . . . . . . . . . . . . . . . . 87

4.9 Observed number of data events for regions A, B, and C. The optimisation procedure for the final three cuts is explained in the previous section. . . . . . . . . . 93 
4.10 Expected number of background events for regions A, B, and C. Recall that the last three cuts are optimised differently for each of the regions. . . . . . . . . . . 94

4.11 Expected number of background events from Standard Model W production processes. Only statistical uncertainties are shown. . . . . . . . . . . . . . . 95

4.12 Expected number of background events from Standard Model Z production processes. Only statistical uncertainties are shown. . . . . . . . . . . . . 96

4.13 Expected number of background events for $t \bar{t}$, WW and QCD multijet processes. Only statistical uncertainties are shown. . . . . . . . . . . . . . . . . 97

B.1 The center column is the number events passing the L1-MET25 trigger path along with any cut shown on the left column. The right column shows the number of events in the center column including the L2 TWO-JET10 trigger requirement. The numbers shown are not prescaled. . . . . . . . . . . . . . . . . 123 


\section{Chapter 1}

\section{Introduction}

Along the history, people has always been wondering what is the world made of. What are the constituents of all the things that we can see in nature and what are the laws that govern their behaviour. This is precisely the goal of elementary particle physics: identify the fundamental building blocks of our world and describe their interactions.

The Standard Model (SM) is currently the most comprehensive theoretical framework that describes the physics related to the elementary particles. The model describes three of the four fundamental forces (gravity is not included) between particles: electromagnetism, weak and strong. All these forces are mediated by carrier particles which obey Bose-Einstein statistics and are called gauge bosons. The model also encloses the matter constituents of the universe which are particles called fermions which follow Fermi-Dirac statistics. There are two fundamentally different types of fermions: quarks and leptons. They both interact via the electroweak force but only the quarks feel the strong force. There are six type of quarks and six type of leptons and they are all arranged in three groups or families with certain properties. In addition, all the fundamental particles which constitute matter have a partner with opposite charge that form the antimatter.

The model is built on the basis of different symmetries observed in nature. Until now, no deviation has been found between the experimental measurements and the SM predictions to an astonishing level of precision. Nevertheless, the SM cannot be the ultimate theory since it includes a rather large number of free parameters and suffers from severe theoretical difficulties at higher energies.

Supersymmetry (SUSY) is broadly considered as one of the most probable extensions of the SM. SUSY introduces a new symmetry which relates masses and couplings of bosons and fermions via spin- $1 / 2$ charges. In this way, for every existing boson in the SM it must exist a fermionic super-partner (named with a suffix "ino"), and likewise, for every fermion a bosonic super-partner (named with a prefix "s") must also exist. Moreover, another symmetry called R- 
parity is introduced to prevent baryon and lepton number violating interactions. If R-parity is conserved, super-particles can only be pair-produced and they cannot decay completely in SM particles. This implies the existence of a lightest SUSY particle (LSP) which would provide a candidate for cold dark matter in our universe, as it is strongly suggested by recent astrophysical data.

SUSY particles have not been found yet, hence supersymmetry must be a broken symmetry. Nevertheless, there are some hints which indicate that if there exist super-partners of the SM particles, they must be at the TeV energy frontier. In Run I at the Tevatron at Fermilab, protons and anti-protons collided at $1.8 \mathrm{TeV}$. This opened the possibility to find some of these new particles, confirming the theory. These particles were not found but some limits to its existence were established providing more constraints to future studies. In Run II, the Tevatron and the CDF detector were upgraded and, among other important things, the center-of-mass energy was increased to $1.96 \mathrm{TeV}$. The good performance of the accelerator and the detector translates into larger data samples that open the possibility of finding new physics.

Since these new particles are very massive, they decay into cascades of high transverse momentum jets. If R-parity is conserved, a large missing transverse energy signal is observed due to the presence of two LSPs that leave the detector undetected.

In this $\mathrm{PhD}$ thesis, the existence of the gluon and quarks super-partners (gluino and squarks, respectively) will be investigated using CDF Run II samples of events with large missing transverse energy and multi-jets in the final state. Chapter 2 is devoted to present the theoretical framework in which SUSY is introduced, together with the motivations and the particular characteristics of the symmetry breaking. Chapter 3 describes the accelerator and detector characteristics and chapter 4 is the one describing in detail the analysis performed and the results obtained. Finally, a discussion of the results and some ideas for future analyses are stated in chapter 5. 


\section{Chapter 2}

\section{Introduction to Supersymmetry}

\subsection{The Standard Model Framework}

The SM is the most compelling and precise model to understand particles and their interactions that we currently have. A complete discussion of the SM is broadly available in the literature [1]. Briefly, the SM is a quantum field theory which describes the unification of electromagnetic and weak interactions into an electroweak sector, and contains a Quantum Chromodynamics (QCD) sector for the description of the strong interactions. However, as it will become clear later, the SM cannot be the ultimate theory to describe particles and their interactions in nature because some aspects remain obscure.

\subsubsection{The SM content}

The fermionic sector of the SM consists of quarks and leptons and it is organised in three families (generations) with identical properties except for the mass, as it is shown in Tab. 2.1. In addition, Tab. 2.2 shows the different gauge bosons available within the SM framework, which are responsible of three of the four main forces present in nature. Gravity is the force not included in the SM and this is one of the main motivations for searches of theories beyond.

The $\mathrm{SM}$ is based on the symmetry group $\mathrm{SU}(3)_{C} \otimes \mathrm{SU}(2)_{L} \otimes \mathrm{U}(1)_{Y}$, where $C$ denotes colour, $L$ chirality and $Y$ hypercharge. Every group has a coupling constant associated with: $g_{S}$ (related with $\alpha_{s}$ ) for the strong interactions; $g$ (related with the Fermi constant $G_{F}$ ) for the weak interactions and $g^{\prime}$ (related with the electron charge $e$ ) for the electromagnetic interactions.

In the following sections, the different theories that conform the basis of the SM formulation are briefly presented. 


\begin{tabular}{|c|c|c|c|}
\hline SM Fermions & 1st Generation & 2nd Generation & 3rd Generation \\
\hline \hline \multirow{3}{*}{ QUARKS } & Up $(\mathbf{u})$ & Charm $(\mathbf{c})$ & Top $(\mathbf{t})$ \\
& $1.5-3.0 \mathrm{MeV} / \mathrm{c}^{2}$ & $1.25 \pm 0.09 \mathrm{GeV} / \mathrm{c}^{2}$ & $172.5 \pm 1.3 \pm 1.9 \mathrm{GeV} / \mathrm{c}^{2}$ \\
\cline { 2 - 4 } & Down $(\mathbf{d})$ & Strange $(\mathbf{s})$ & Bottom $(\mathbf{b})$ \\
& $3-7 \mathrm{MeV} / \mathrm{c}^{2}$ & $95 \pm 25 \mathrm{MeV} / \mathrm{c}^{2}$ & $4.20 \pm 0.07 \mathrm{GeV} / \mathrm{c}^{2}$ \\
\hline \hline \multirow{3}{*}{ LEPTONS } & Electron neutrino ${ }^{1}\left(v_{\mathbf{e}}\right)$ & Muon neutrino ${ }^{1}\left(v_{\mu}\right)$ & Tau neutrino $\left(v_{\tau}\right)$ \\
\cline { 2 - 4 } & $<2 \mathrm{eV} / \mathrm{c}^{2}$ & $<0.19 \mathrm{MeV} / \mathrm{c}^{2}$ & $<18.2 \mathrm{MeV} / \mathrm{c}^{2}$ \\
& Electron $(\mathbf{e})$ & Muon $(\mu)$ & Tau $(\tau)$ \\
& $0.511 \mathrm{MeV} / \mathrm{c}^{2}$ & $105.66 \mathrm{MeV} / \mathrm{c}^{2}$ & $1776.99_{-0.26}^{+0.29} \mathrm{GeV} / \mathrm{c}^{2}$ \\
\hline
\end{tabular}

Tab. 2.1: Fermionic sector of the SM. Masses are taken from the Particle Data Group (PDG) [2]. Top mass quoted is the current best estimate for the "pole" mass.

\begin{tabular}{|c|c|c|}
\hline Particle & Mass & Interaction \\
\hline \hline Gluon $(g)$ & 0 & strong/colour SU $(3)_{C}$ \\
\hline Photon $(\gamma)$ & 0 & electromagnetic $\mathrm{U}(1)_{e m}$ \\
\hline$Z$ & $91.188 \pm 0.002 \mathrm{GeV} / \mathrm{c}^{2}$ & weak neutral \\
$W^{ \pm}$ & $80.403 \pm 0.029 \mathrm{GeV} / \mathrm{c}^{2}$ & weak charged \\
\hline \hline
\end{tabular}

Tab. 2.2: Standard Model gauge bosons and the corresponding interactions. Masses are taken from the Particle Data Group (PDG) [2].

\subsubsection{Quantum Electrodynamics}

Quantum Electrodynamics (QED) was developed in the late 1940s and early 1950s chiefly by Feynman, Schwinger and Tomonaga, to describe the electromagnetic interactions of electrons and photons. This is a quantum relativistic renormalisable theory which is invariant under a change of phase or gauge, $\theta$ :

$$
\psi \rightarrow \psi^{\prime}=e^{i Q \theta} \psi
$$

where $Q$ represents the charge and $\psi$ is the Dirac field (spin 1/2).

In order to promote the global symmetry under U(1) transformations, responsible for the con-

\footnotetext{
${ }^{1}$ These limits are approximations since the fact that in the mixing matrix there exist two large mixing angles prevents from assuming a "dominant eigenstate" approximation as in the case of the CKM matrix. For details see [2].
} 
servation of the charge, to a local one $(\theta=\theta(x))$, the covariant derivative needs to be introduced:

$$
D_{\mu} \equiv \partial_{\mu}-i e Q A_{\mu},
$$

where $A_{\mu}$ is a field that satisfies:

$$
A_{\mu} \rightarrow A_{\mu}^{\prime} \equiv A_{\mu}+\frac{1}{e} \partial_{\mu} \theta .
$$

Therefore, the lagrangian describing the theory becomes:

$$
\left\llcorner=\bar{\psi}\left(i \gamma^{\mu} D_{\mu}-m\right) \psi=\bar{\psi}\left(i \gamma^{\mu} \partial_{\mu}-m\right) \psi+\iota_{I}\right.
$$

where the last term corresponds to the interaction with the new field, $A_{\mu}$ :

$$
\iota_{I}=e Q A_{\mu}\left(\bar{\psi} \gamma^{\mu} \psi\right)
$$

In addition, the kinetic energy of the new field needs to be introduced. From Maxwell's equations, the kinetic term must be of the form:

$$
\mathcal{L}_{K}=-\frac{1}{4} F_{\mu v} F^{\mu v}
$$

where $F_{\mu v} \equiv \partial_{\mu} A_{v}-\partial_{v} A_{\mu}$.

Thus, in this theory the electromagnetic interaction is described by two quantum fields: one for the charged particles and one for the photon. The strength of the interaction is usually described by the coupling constant $\alpha_{\mathrm{e} m}$ whose value depends on the momentum transfer $q^{2}$ in an interaction. At $q^{2} \rightarrow 0$ (or low energies) the coupling constant value is that of the fine structure constant, $\alpha_{\mathrm{e} m}=\frac{e^{2}}{4 \pi / h c}=\frac{1}{137}$. At the scale of the Z-boson (short distances), its value increases: $\alpha_{\mathrm{e} m}\left(m_{Z}\right) \approx \frac{1}{128}$.

\subsubsection{Electroweak theory}

The weak theory was proposed by Enrico Fermi in 1934 in order to explain the proton $\beta$-decay. In this theory four fermions directly interacted with one another in such a way that a neutron (or a down-quark) could be directly splitted into an electron, an antineutrino and a proton (an up-quark). The strength of the Fermi's interaction was given by the Fermi constant, $G_{F}$.

Feynman diagrams described the interaction remarkably well at tree level but loop diagrams could not be calculated reliably because Fermi's interaction was not renormalisable. The solution came in 1967 when the electromagnetic and weak interactions were successfully unified by Glashow, Salam and Weinberg [3]. This unification constituted the Standard Electroweak Model which is the core of the SM. The idea of the unification is to combine both interactions into one single theoretical framework in which they would appear as two manifestations of the same fundamental interaction. These interactions are unified under the group $\mathrm{SU}(2)_{L} \otimes \mathrm{U}(1)_{\mathrm{Y}}$. The first part 
of the group has dimension three and therefore, three generators are needed: $t_{i}=\frac{\sigma_{i}}{2}(\mathrm{i}=1,2,3)$ where $\sigma_{i}$ are the Pauli matrices. These generators, due to the global gauge invariance under $\mathrm{SU}(2)$, introduce a new quantum number called the weak isospin $(T)$. This number is associated to the different spin-like multiplets. Since weak force only interacts with left-handed particles (righthanded antiparticles), the left-handed fermions transform as doublets while the right handed ones transform as singlets:

$$
\begin{aligned}
& f_{L}^{i}=\left(\begin{array}{c}
v_{L}^{i} \\
l_{L}^{i}
\end{array}\right), \quad\left(\begin{array}{c}
u_{L}^{i} \\
d_{L}^{i}
\end{array}\right) \\
& f_{R}^{i}=l_{R}^{i}, u_{R}^{i}, d_{R}^{i}
\end{aligned}
$$

where $i=1,2,3$ corresponds to the family index. Hence, the weak interaction is divided into a "charged part" (that is, exchanging the components of the doublet) and a "neutral part" (that is, leaving the doublets as they are). Since SU(2) is a non-Abelian group, it allows self-interactions of these gauge fields.

Since the group $U(1)_{Y}$ has only one dimension, its structure is more simple having only one generator called the hypercharge $\hat{Y}$. Once the $\mathrm{SU}(2)_{L} \otimes \mathrm{U}(1)_{Y}$ group is defined, the SM electroweak lagrangian is obtained by requiring invariance under local gauge transformations to obtain an interacting field theory, following the analogy with QED. This is achieved by replacing the derivatives of the fields by the corresponding covariant derivative, which now has the form:

$$
D_{\mu} \equiv \partial_{\mu}-i g \vec{T} \vec{W}_{\mu}-i g^{\prime} \frac{Y}{2} B_{\mu}
$$

where g and g' are the coupling constants corresponding to $S U(2)_{L}$ and $U(1)_{Y}$, respectively.

Then, the electroweak lagrangian can be written as:

$$
\mathcal{L}_{S M}=\mathcal{L}_{f}+\mathcal{L}_{G}+\mathcal{L}_{S S B}+\mathcal{L}_{Y W} .
$$

The first term corresponds to the fermion lagrangian:

$$
{ }^{\iota_{f}}=\sum_{f=l, q} \bar{f} i \not D f
$$

The second term is the contribution from the gauge fields:

$$
{ }_{\iota_{G}}=-\frac{1}{4} W_{\mu v}^{i} W_{i}^{\mu v}-\frac{1}{4} B_{\mu v} B^{\mu v}+\iota_{G F}+\mathcal{L}_{F P},
$$


where $W_{\mu v}^{i}$ (with $i=1,2,3$ ) and $B_{\mu v}$ are, respectively, the field strength tensors for $S U(2)_{L}$ and $U(1)_{Y}$ defined as:

$$
\begin{aligned}
W_{\mu \nu}^{i} & \equiv \partial_{\mu} W_{v}^{i}-\partial_{v} W_{\mu}^{i}+g \varepsilon^{i j k} W_{\mu}^{j} W_{v}^{k} \\
B_{\mu \nu} & \equiv \partial_{\mu} B_{v}-\partial_{v} B_{\mu}
\end{aligned}
$$

and $\mathcal{L}_{G F}$ and $\mathcal{L}_{F P}$ are the gauge fixing and Faddeev Popov lagrangians that are needed in any theory [4].

The last two terms of the electroweak lagrangian Eq. (2.10) are the symmetry breaking sector and the Yukawa lagrangian, respectively, which will be described in next subsection.

The gauge fields presented at Eq. (2.13) can be rewritten as:

$$
\begin{aligned}
W_{\mu}^{ \pm} & =\frac{1}{\sqrt{2}}\left(W_{\mu}^{1} \mp i W_{\mu}^{2}\right) \\
Z_{\mu} & =\cos \theta_{W} W_{\mu}^{3}-\sin \theta_{W} B_{\mu} \\
A_{\mu} & =\sin \theta_{W} W_{\mu}^{3}+\cos \theta_{W} B_{\mu}
\end{aligned}
$$

where, again, $A_{\mu}$ represents the photon field and $\cos \theta_{W}=\frac{g}{\sqrt{g^{2}+g^{2}}}$ is the weak mixing angle, which relates both couplings by the simple relation $\tan \theta_{W}=g^{\prime} / g$. In addition, $W_{\mu}^{ \pm}$and $Z_{\mu}$ fields are associated to the physical $W^{ \pm}$and $Z^{0}$ boson particles. In this framework, the electron charge and the Fermi constant can be written in terms of the couplings through the following relations:

$$
\begin{aligned}
e & =g \sin \theta_{W} \\
G_{F} & =\frac{\sqrt{2}}{8} \frac{g^{2}}{m_{W}^{2}} .
\end{aligned}
$$

The electric charge $\hat{Q}$, the third component of the weak isospin $\hat{T}_{3}$, and the weak-hypercharge $\hat{Y}$ are linearly related by the Gell-Mann Nishijima formula:

$$
\hat{Q}=\hat{T}_{3}+\hat{Y} / 2 \text {. }
$$

Hence, the global and local conservation of weak-isospin and hypercharge naturally implies charge conservation, as required by QED, and the electromagnetic and weak interactions are unified under the same theoretical framework.

\subsubsection{The Higgs mechanism}

As shown, the Standard Model formalism allows the unification of electromagnetic and weak interactions through the exploitation of a local gauge symmetry. Nevertheless, this gauge symmetry 
requires massless $W^{ \pm}$and $Z$ bosons. This requirement is in contradiction with the observation and one needs to introduce a mechanism for generating non-zero masses while preserving the renormalisability of the theory. In the SM, the Higgs mechanism of Spontaneous Symmetry Breaking (SSB) is proposed.

In the SSB, one introduces a new field, the Higgs field, such as:

$$
\Phi \equiv\left(\begin{array}{c}
\phi^{+} \\
\phi^{0}
\end{array}\right)
$$

The correspondent kinetic and potential term in the lagrangian have the form:

$$
{ }_{L_{\Phi}}=\left(D_{\mu} \Phi\right)^{\dagger} D^{\mu} \Phi-V(\Phi),
$$

where

$$
V(\Phi)=\mu^{2} \Phi^{\dagger} \Phi+\lambda\left(\Phi^{\dagger} \Phi\right)^{2}
$$

If $\lambda>0$ and $\mu^{2}<0$ the potential $V(\Phi)$ has a minimum for:

$$
\Phi^{\dagger} \Phi=-\frac{\mu^{2}}{2 \lambda} \equiv \frac{v^{2}}{2}
$$

Thus, the field $\Phi$ has a non-zero vacuum expectation value (VEV): $\left(<0|\Phi| 0>=\frac{v}{\sqrt{2}} \neq 0\right)$. Choosing one of a set of degenerate states of minimum energy breaks the gauge symmetry.

As stated by the Goldstone theorem, fields that acquire a VEV will have an associated massless Goldstone boson which will disappear transformed into the longitudinal component of a massive gauge boson. Since the photon is known to be massless, the symmetry is chosen to be broken so that only the fields with zero electric charge (the ones that cannot couple to the electromagnetic interaction) acquire a VEV. In such a way, the symmetry of the photon-associated operator, $\hat{Q}$ is preserved:

$$
\begin{gathered}
\Phi_{0} \equiv<0|\Phi| 0>\equiv\left(\begin{array}{l}
0 \\
v
\end{array}\right) \\
\hat{Q} \Phi_{0}=0 .
\end{gathered}
$$

Expanding around the true minimum of the theory, the complex field $\phi$ becomes:

$$
\Phi(x)=e^{i \frac{\vec{i} \vec{\xi}(x)}{2}} \frac{1}{\sqrt{2}}\left(\begin{array}{c}
0 \\
v+H(x)
\end{array}\right) .
$$

where the three parameters $\vec{\xi}(x)$ correspond to the motion through the degenerated minima in the $\mathrm{SU}(2)$ space. Since the lagrangian is locally gauge invariant, one can choose $\vec{\xi}(x)=0$. Hence, 
introducing this expansion into the SM lagrangian Eq. (2.10), one obtains tree level predictions for massive fermions (coming from the $\iota_{Y W}$ part), massive gauge bosons (coming from the kinetic part of $\mathcal{L}_{S S B}$ and a new Higgs boson. These relations are:

$$
\begin{aligned}
M_{W} & =\frac{v g}{2} ; \quad M_{Z}=v \frac{\sqrt{g^{2}+g^{\prime 2}}}{2} \\
M_{H} & =\sqrt{-2 \mu^{2}}=\sqrt{2 \lambda} v \\
m_{f} & =\lambda_{f} \frac{v}{\sqrt{2}} \\
m_{\gamma}^{2} & =0
\end{aligned}
$$

where $f$ stands for the fermions in the theory. These relations can also be expressed in function of the weak mixing angle,

$$
M_{z}=\frac{\frac{1}{2} v g}{\cos \theta_{W}}
$$

which leads to the SM prediction

$$
\frac{M_{W}^{2}}{M_{Z}^{2}}=\cos ^{2} \theta_{W}
$$

This prediction was tested once the $W^{ \pm}$and $Z$ vector bosons where discovered in 1983 by UA1 and UA2 collaborations at the CERN SPS [5].

The ten independent fields before SSB (three massless gauge bosons $\left(W^{ \pm}, Z\right)$, with two polarisation states each, and one $\mathrm{SU}(2)$ doublet of complex scalars) are now represented by three massive bosons, which account for nine degrees of freedom, and a new physical scalar particle called the Higgs boson, which accounts for the last one.

This new particle, which is the missing piece to confirm the Higgs mechanism, has the couplings completely defined by the theory:

$$
\lambda_{H H H}=3 \frac{M_{H}^{2}}{M_{Z}^{2}} ; \quad \lambda_{H V V}=2 \sqrt{2 G_{F}} M_{V}^{2} ; \quad \lambda_{H f f}=2 \sqrt{2 G_{F}} m_{f},
$$

where $V=W, Z$ and $G_{F}$ is the Fermi constant. The vacuum expectation value $v$ is determined experimentally from the partial width $\Gamma\left(\mu \rightarrow \mathrm{v}_{\mu} \overline{\mathrm{v}}_{e} e\right)$ at low energies $\left(q^{2}<<M_{W}^{2}\right)$ :

$$
\frac{G_{F}}{\sqrt{2}}=\frac{g^{2}}{8 M_{W}^{2}}=\frac{1}{2 v^{2}},
$$

where, substituting experimental values:

$$
v=\left(\sqrt{2} G_{F}\right)^{-\frac{1}{2}}=246 \mathrm{GeV},
$$


which sets the electroweak symmetry breaking scale.

This new particle allows Yukawa-like terms in the lagrangian:

$$
g_{f}\left[\left(\bar{f}_{L} \phi\right) f_{R}+\text { h.c. }\right]
$$

which can be written in terms of the VEV:

$$
\sqrt{\frac{1}{2}} g_{f} v\left(\bar{f}_{L} f_{R}+\bar{f}_{R} f_{L}\right)
$$

Therefore, not only the bosons acquire mass through the Higgs mechanism but also the fermions with $m_{f}=g_{f} v / \sqrt{2}$. Noticeably, the strength of the coupling is proportional to the masses. However, masses are not predicted unless $g_{f}$ is determined.

\subsubsection{Quantum Chromodynamics}

Quantum Chromodynamics (QCD) [6] was developed in 1973 to describe the behaviour of quarks being held together by the strong force carried by gluons. Again, quantum field theory is the framework in which QCD is developed. In this case, the "colour" group $\mathrm{SU}(3)_{C}$ is the starting global symmetry. This new quantum number (colour) is introduced to refer the three possible states of the quarks and it constitutes an exact symmetry (the particular colour of the quarks is not affecting the dynamics of a process). In order to promote the global symmetry to a local one, the covariant derivatives of the fields take the form:

$$
D_{\mu} q \equiv\left(\partial_{\mu}-i g_{s}\left(\frac{\lambda_{\alpha}}{2}\right) A_{\mu}^{\alpha}\right) q
$$

where $g_{s}$ is the strong coupling constant, although this name is usually reserved for references to $\alpha_{s}$ since there is a direct relation between them: $g_{s} \equiv 4 \pi \alpha_{s}$. In addition, $\frac{\lambda_{\alpha}}{2}$ are the SU(3) generators (with $\alpha=1,2, \cdots, 8$ ), $A_{\mu}^{\alpha}$ are the gluon fields and $q$ is a vector of three components corresponding to the different colours.

The QCD lagrangian is written in terms of the quarks, their covariant derivatives and the kinetic term for the gluons fields:

$$
{ }^{\llcorner} \mathrm{QCD}=\sum_{q} \bar{q}(x)\left(i \not D-m_{q}\right) q(x)-\frac{1}{4} F_{\mu v}^{\alpha}(x) F_{\alpha}^{\mu v}(x)
$$

where $F_{\mu v}^{\alpha}(x)$ is the gluon field strength, which unlike the QED case, is given by,

$$
F_{\mu v}^{\alpha}(x)=\partial_{\mu} A_{v}^{\alpha}(x)-\partial_{v} A_{\mu}^{\alpha}(x)+g_{S} f^{\alpha \beta \gamma} A_{\mu \beta}^{\alpha} A_{v \gamma}^{\alpha},
$$


where $f^{\alpha \beta \gamma}$ are the structure constants of the $\mathrm{SU}(3)_{C}$ group.

Similarly to QED, the gauge interactions among the quarks and gluons are contained in the $\bar{q} i \not D q$ term,

$$
\bar{q} g_{s} \frac{\lambda^{\alpha}}{2} A_{\mu}^{\alpha} \gamma^{\mu} q
$$

However, there is an important difference with the QED case. The gluon kinetic term $F_{\mu \nu}^{\alpha} F_{\alpha}^{\mu v}$ contains a three and a four gluon term, which are precisely the self-interaction gluon vertices characteristic of a non-abelian theory. These cubic and quartic terms are the responsible for the true nature of the strong force. When a pair of quarks begin to separate from each other, the exchanged gluons interact with each other and the strong coupling constant, $\alpha_{s}$, increase. This increasing force either binds the quarks together at low-energy scale (large distances) or it breaks when the energy density of the colour field between the quarks is great enough to create a quarkantiquark pair, resulting in two separate hadrons ${ }^{2}$. This situation is called quark confinement. On the opposite side, at high energies (small distances), the strong interaction proceeds via colour fields of reduced strength and the quarks and gluons behave as essentially free. This situation is called asymptotic freedom. The amplitude of a strong interaction process at a given momentum scale, $q^{2}$, can be parameterised in terms of the running coupling constant $\alpha_{s}(q)$. A conventional definition of $\alpha_{s}$, at leading order (LO), is given by [7]:

$$
\alpha_{s}(q)=\frac{4 \pi}{\left(11 n_{c}-2 n_{f}\right) \ln \left(q^{2} / \Lambda^{2}\right)}
$$

where $n_{c}\left(n_{f}\right)$ is the number of colours (flavours) of the quarks with mass less than the energy scale $q$ and $\Lambda$ is the QCD scale, which is the only adjustable parameter of QCD and depends on the momentum scale of the interaction. It marks the energy scale at which $\alpha_{s}$ becomes large and the perturbative approach is no longer valid. For most processes, the measured value of $\Lambda_{Q C D}$ is consistent with $200 \mathrm{MeV} / \mathrm{c}$. The running strong coupling presented in Eq. (2.40) shows that for large $q^{2}$ (small distances), the coupling becomes small (asymptotic freedom) but at low $q^{2}$, the coupling approaches to unity. Hence, high- $q^{2}$ processes can be described by perturbative calculations but low- $q^{2}$ interactions need to rely on phenomenological model, as will be described in section 2.1.9. The value of $\alpha_{s}$ at the $\mathrm{Z}$ pole mass is: $\alpha_{s}\left(q=m_{Z}\right)=0.1176 \pm 20$ [2].

\subsubsection{QCD description of the hadrons}

Hadrons are not elementary particles but constituted by quarks and gluons, usually referred by the generic name "partons". In the description of the parton kinematics inside hadrons two variables are usually used, originally defined in the context of deep inelastic scattering (DIS) experiments:

\footnotetext{
${ }^{2}$ The top quark constitutes an exception in the sense that due to its huge mass, it decays before it can hadronise.
} 


$$
Q^{2} \equiv\left(k-k^{\prime}\right)^{2} \quad \text { and } \quad x \equiv \frac{Q^{2}}{2(p \cdot q)},
$$

where $k\left(k^{\prime}\right)$ is the 4-momentum of the ingoing (outgoing) electron and $p$ is the 4-momentum of the incoming proton. Therefore $Q^{2}$ is the energy scale of the interaction and $x$, named the Bjorken variable, can be interpreted as the fraction of the proton 4-momentum carried by the struck quark.

The probability of finding a certain parton within the hadron carrying a particular fraction $x$ of the hadron momentum is given by a parton distribution function (PDF), which presents a logarithmic dependence on $Q^{2}$.

The factorisation theorem allows to separate long-distance and short-distance processes. A cross section for a hard scattering process initiated by two hadrons with four-momenta $P_{1}$ and $P_{2}$ can be written as:

$$
\sigma\left(P_{1}, P_{2}\right)=\sum_{i, j} \int d x_{1} d x_{2} f_{i}\left(x_{1}, \mu_{F}^{2}\right) f_{j}\left(x_{2}, \mu_{F}^{2}\right) \hat{\sigma}_{i j}\left(p_{1}, p_{2}, \alpha_{s}\left(\mu_{F}^{2}\right), Q^{2} / \mu_{F}^{2}\right)
$$

where the momenta of the partons which participate in the hard interaction are $p_{1}=x_{1} P_{1}$ and $p_{2}=x_{2} P_{2}$. The $\hat{\sigma}_{i j}$ is the parton-parton cross section and $f_{i}\left(x_{1}, \mu_{F}^{2}\right)$ is the PDF defined at a factorisation scale $\mu_{F}$, which is used to separate the soft and the hard processes. Therefore, any parton emitted with small transverse momentum, less than $\mu_{F}$, is considered part of the hadron structure and is absorbed into the parton distribution. By doing that, the PDFs are independent of the hard process, which ensures their universality.

Although the perturbative QCD (pQCD) is not able to predict the values of the PDF at a given $x$, it is able to describe the evolution of the PDFs as a function of $Q^{2}$. The processes that generate the parton interactions to first order in $\alpha_{s}$ are gluon radiation $(q \rightarrow q g)$, gluon splitting $(g \rightarrow g g$ ) and quark pair production $(g \rightarrow q \bar{q})$. Each of these processes have associated a splitting function $P_{p^{\prime}}(x / z)$ which represents the probability that a parton of type $p$ converts into a parton of type $p^{\prime}$, carrying a fraction $x / z$ of the momentum of the original parton $p$.

The particular expressions of the splitting functions can be calculated in pQCD and the evolution of the parton densities in $Q^{2}$ can be written in terms of these splitting functions:

$$
\begin{aligned}
\frac{d q_{i}\left(x, Q^{2}\right)}{d \log \left(Q^{2}\right)} & =\frac{\alpha_{s}}{2 \pi} \int_{x}^{1}\left(q_{i}\left(z, Q^{2}\right) P_{q q}\left(\frac{x}{z}\right)+g\left(z, Q^{2}\right) P_{q g}\left(\frac{x}{z}\right)\right) \frac{d z}{z}, \\
\frac{d g\left(x, Q^{2}\right)}{d \log \left(Q^{2}\right)} & =\frac{\alpha_{s}}{2 \pi} \int_{x}^{1}\left(\sum_{i} q_{i}\left(z, Q^{2}\right) P_{g q}\left(\frac{x}{z}\right)+g\left(z, Q^{2}\right) P_{g g}\left(\frac{x}{z}\right)\right) \frac{d z}{z},
\end{aligned}
$$

where the first equation describes the change of the quark densities with $Q^{2}$ due to gluon radiation and gluon splitting and the second equation describes the change of the gluon density 
with $Q^{2}$ due to gluon radiation off quarks and gluons. These equations are called the DGLAP (Dokshitzer, Grobov, Livatov, Altarelli and Paris) equations [8]. The equations assume massless partons. Hence, they are only valid for gluons and the light quarks ( $u, d$ and $s$ ).

pQCD is not able to predict the $x$ dependence of the PDFs. Hence, data from different experiments is used to parameterise the PDFs at a starting scale $Q_{0}^{2}$. Then, the predictions for each parton density at a higher scale $Q^{2}$ are obtained using the DGLAP evolution equations.

\subsubsection{PDF parameterisations}

The understanding of the PDFs plays a fundamental role on interpreting the data at hadron colliders in terms of the SM predictions and possible deviations. The parameterisation of the PDFs at a certain scale $Q_{0}^{2}$ is carried out through a $\chi^{2}$ minimisation over data from different processes such as deep-inelastic $e, \mu$ or $v$ scattering, Drell-Yan production, $W$-asymmetry in $p \bar{p}$ collisions and prompt photon production $p N \rightarrow \gamma X$. Different groups perform such parameterisations. In the work presented here, the parameterisations done by the 'Coordinated Theoretical-Experimental Project on QCD' (CTEQ Collaboration) are the ones used. The CTEQ collaboration has modified the functional forms for quarks and gluon distributions to accommodate latest results from the Tevatron Run I. The most recent set from CTEQ collaboration is the CTEQ6 where the following form has been chosen [9]:

$$
x f\left(x, Q_{0}^{2}\right)=A_{0} \cdot x^{A_{1}}(1-x)^{A_{2}} e^{A_{3}} x\left(1+e^{A_{4}} x\right)^{A_{5}},
$$

where $A_{i}$ are the parameters to be fitted and $f$ are the quarks and gluon distribution functions. An example of these PDF's can be seen in Fig. 2.1 at two different scales $(Q=2 \mathrm{GeV}$ and $Q=100 \mathrm{GeV})$.

\subsubsection{PDF uncertainties}

To evaluate the uncertainties on the fit, a Hessian method based on the up and down variation of the parameters in the PDFs fits has been developed. Some details are given in Section 4.6 when this method is applied to determine the PDF uncertainties on the analysis. In any case, this method determines the behaviour of the $\chi^{2}$ in the neighbourhood of the minimum. Variations on the set of PDF parameters lead to new fits with certain $\chi^{2}$. A parameter called tolerance, $T$, is defined and the new fits are considered acceptable if $\chi^{2}-\chi_{0}^{2}<T^{2}$, where $\chi_{0}^{2}$ is the best fit to the global data set. CTEQ choses $T^{2} \sim 100$ which is interpreted to be a $90 \%$ C.L. uncertainty.

In Fig. 2.2 the uncertainties on gluon and $u$-quark distributions are shown at a scale $Q^{2}=10$ $\mathrm{GeV}^{2}$. The u-quark distribution is tightly constrained for $x \leq 0.8$. The gluon uncertainty is of order $\pm 15 \%$ for $x \leq 0.3$ and then it increases rapidly for large $x$. These uncertainties also increase 

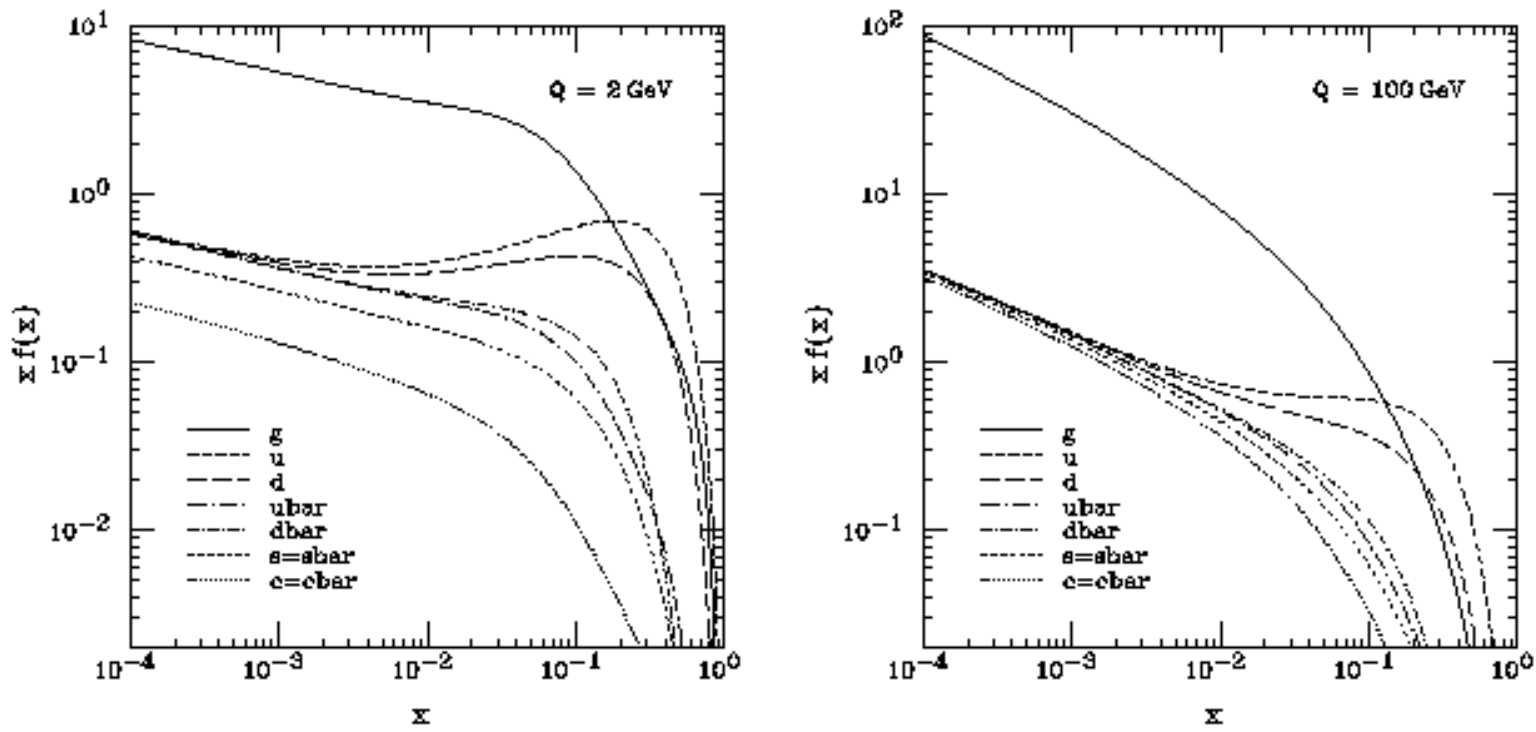

Fig. 2.1: CTEQ6 parton distribution functions at $Q=2 \mathrm{GeV}$ and $Q=100 \mathrm{GeV}$.

at higher energy scales when applying the DGLAP evolution equations. This will constitute one of the main systematic uncertainties in the analysis presented here.
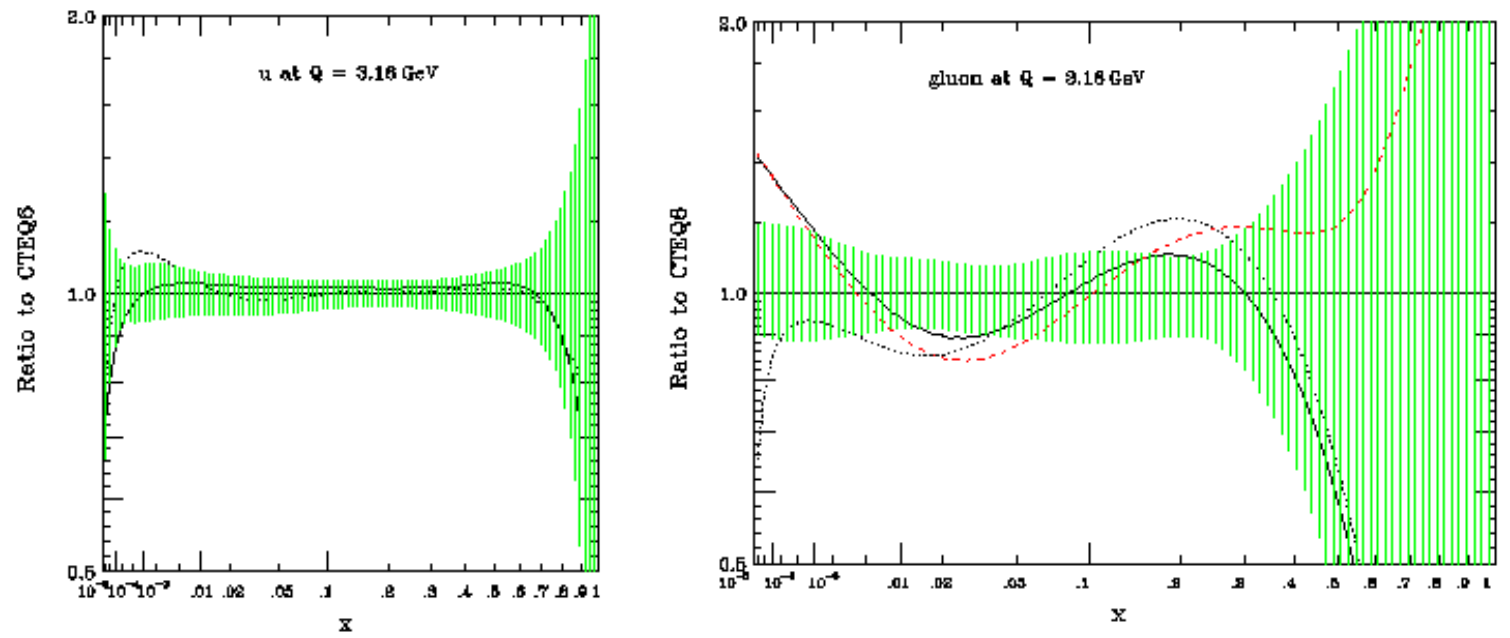

Fig. 2.2: Uncertainty bands for the $u$-quark (left) or gluon (right) distribution functions at $Q^{2}=10 \mathrm{GeV}^{2}$. The other lines are from different type of parameterisations not discussed here. 


\subsection{9 $p \bar{p}$ collisions}

In a typical high energy proton-antiproton collision, several physics processes play an important role. The knowledge of the different aspects of the collision is crucial for a proper understanding of the resulting event. Although QCD does not allow to strictly separate the different processes, the following steps implemented in Monte Carlo (MC) models, also shown in Fig. 2.3, provide a useful approach to understand what happens in such collisions:

- Initially two beam particles are coming in towards each other. Each particle is characterised by a set of parton distributions, which defines the partonic substructure in terms of flavour composition and energy sharing.

- One shower initiator parton from each beam starts off a sequence of gluon radiation, such as $q \rightarrow q g$, which build up an initial-state shower (ISR).

- One incoming parton from each of the two showers is involved in the hard scattering process, a 2-to- 2 process, that can be calculated by a perturbative approach to first-order.

- The outgoing partons radiate gluons, just like the incoming did, to build up final-state showers (FSR).

- Further semihard interactions may occur between the other partons of the two incoming hadrons.

- The remnants have internal structure and a net colour charge that relates them to the rest of the final state.

- The QCD confinement mechanism ensures that the outgoing quarks and gluons are not observable, but fragment to colour neutral hadrons.

- Many of the produced hadrons are unstable and decay further.

Different Monte Carlo tools have been developed to address some of the processes occuring during a $p \bar{p}$ collision which cannot be calculated completely through $\mathrm{pQCD}$. The parton shower approach and the hadronisation models are presented in the following sections.

\subsubsection{Initial- and Final-State Radiation (ISR/FSR)}

Higher-order QCD processes are approximately implemented in the MC via initial- and final-state parton showers [10]. To describe them, both processes are set to be independent. In a hard process with virtuality $Q^{2}$, initial-state radiation is modelled by a sequence of emissions that, starting from the hadrons, increase the virtuality in each emission until it matches the $Q^{2}$ of the hard process. 


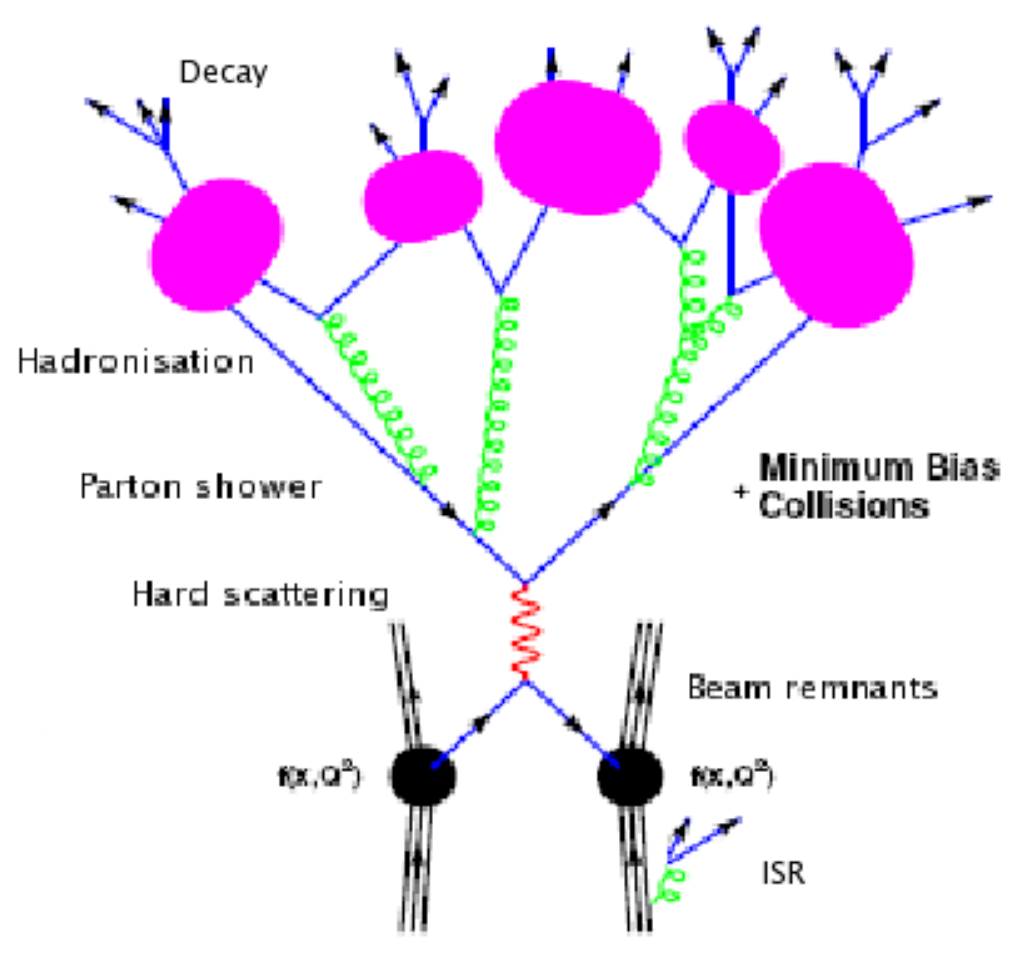

Fig. 2.3: An example of the different processes occurring at a $p \bar{p}$ collision.

Similarly, the final-state radiation is constituted by a sequence of emissions that decreases the virtuality of the partons until a $Q_{0}^{2} \sim \Lambda_{\mathrm{Q} C D}^{2}$ is reached.

The parton shower is characterised by a strong angular ordering of the different emissions, dominated by the colinear component. Although the first branch in the parton shower is approximately performed according to the matrix elements, the collinear approximation will fail in reproducing the hardness of subsequent emissions. For example, PYTHIA 2-to-2 processes will describe the production of a third jet but will produce a too soft fourth jet in the final state.

\subsubsection{Hadronisation}

After the parton shower has finished, the final state consists of a set of partons with virtualities of the order of the cutoff scale $Q_{0}^{2} \sim \Lambda_{\mathrm{Q} C D}^{2}$. QCD becomes strongly interacting at long distances (low momentum-transfer) and non-perturbative effects cannot be neglected. In this confinement regime, the coloured partons are transformed into colourless hadrons in a process called hadronisation or fragmentation. Since this process is still not understood from first principles, some phenomenological models have been constructed to describe it. 


\section{String Fragmentation Model}

The string fragmentation model [11] assumes a linear confinement, i.e. the energy stored in the colour dipole field between a $q$ and a $\bar{q}$ is assumed to increase linearly with the separation between charges. This is a characteristic of QCD interactions due to the presence of a triple-gluon vertex. The physical picture is that of a colour flux vortex line being stretched between a $q$ and a $\bar{q}$ which are moving apart from their common vertex. The transverse dimensions of the tube are of typical hadronic sizes $(\sim 1 \mathrm{fm})$ and the tube is assumed to be uniform along its length. This automatically leads to a confinement picture with a linearly rising potential.

As the $q$ and $\bar{q}$ move apart, the potential energy stored in the string increases and may break producing a new $q^{\prime} \bar{q}^{\prime}$ pair. Hence the system is splitted in two colour-singlet systems $q \bar{q}^{\prime}$ and $q^{\prime} \bar{q}$ from which, depending on their invariant mass, new breaking can occur. In the Lund string model, the string break-up proceeds until only on-mass-shell hadrons remain, each hadron corresponding to a small piece of string. Charm and heavier quarks are not expected to be produced in soft fragmentation, but only in perturbative parton-shower branchings $g \rightarrow q \bar{q}$. If more than two partons are moving apart the string structure becomes more complicated. For a $q \bar{q} g$ event, a string is stretched from the $q$ end via the gluon $(g)$ to the $\bar{q}$ end. To first approximation, there are two fragmenting string pieces holded by the gluon. But additional string regions may appear during the time evolution of the system and complicate the process. Fig. 2.4 shows a schematic diagram of string fragmentation.

\section{Cluster Fragmentation Model}

The cluster fragmentation model [12] is based on an important property of the branching processes which is the colour preconfinement [13]. This property relies on the fact that the separation of the colour charges forming a singlet are inhibited. After the perturbative parton branching process, remaining gluons are splitted into light $q \bar{q}$ pairs. Then, neighbouring quarks and antiquarks can be combined into colour singlets. These singlets have masses distribution and spatial size which peak at low values and are asymptotically independent of the hard subprocess scale. Most clusters have masses of up to few $\mathrm{GeV}$ and it is reasonable to consider them as superpositions of resonances. Clusters decay into hadrons according to two-body phase space. Fig. 2.4 shows a schematic diagram of cluster fragmentation.

\subsection{The limitations of the Standard Model}

The SM description of the different processes involving electroweak or strong interactions is extremely accurate. At the present time, no experiment has been able to find any clear deviation 

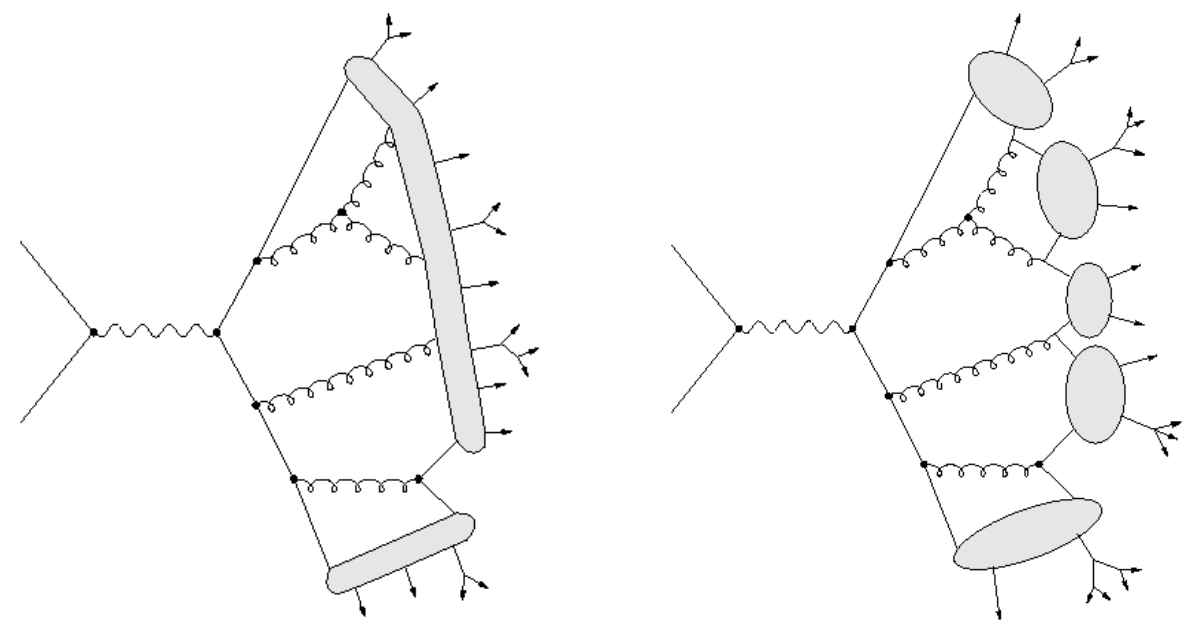

Fig. 2.4: A representation of the string (left) and cluster (right) fragmentation models.

from the SM predictions. Nevertheless, physicists are still pushing to find such tiny deviations. The main reason is that the SM has serious theoretically motivated problems, starting from the fact that gravity is not accommodated in the theory, that prevent it from being the ultimate theory, the Theory of Everything (TOE), that would describe nature in a comprehensive manner.

Even accepting the peculiar set of group representations and hypercharges required by the model, the SM contains at least 19 free parameters, such as couplings, masses and mixings, which cannot be predicted but must be measured by the experiment. In addition, more parameters would be needed if one wants to accomodate non-accelerator observations such as the cosmological baryon asymmetry, neutrino masses and mixings or the problematic cosmological constant.

The SM also leaves several questions unanswered such as why are there three generations, spatial dimensions or colours, how do we understand neutrino oscillations and massive neutrinos, why are the electric charge of the proton and the electron exactly opposite or whether the Higgs mechanism is really the process through which the electroweak symmetry breaking occurs and lay beneath the origin of masses. In addition, the model cannot explain which are the mechanisms to produce the matter-antimatter asymmetry observed in the universe or what is the relation between the strong and electroweak forces.

Perhaps the most surprising feature of the SM is the accurate description of the interactions between particles with masses 17 orders of magnitude smaller than the Planck mass and the difficulty to accommodate gravity within this framework [14]. This feature may be an indication that the SM is an effective theory, that is a "low energy" limit of a more fundamental one. But this assumption automatically leads to the question of up to which energy scale will the SM be valid. 


\subsubsection{The hierarchy problem}

As explained in section 2.1.4, the Higgs mechanism predicts the existence of a scalar particle in the SM physical spectrum, $h_{S M}$. Direct searches of the Higgs at LEP [15] led to the conclusion that it must be heavier than $114.4 \mathrm{GeV} / \mathrm{c}^{2}$ at $95 \%$ C.L. Furthermore, from precision electroweak measurements [16], the Higgs mass is expected to be lower than $M_{h_{S M}} \leq 246 \mathrm{GeV} / \mathrm{c}^{2}$ at $95 \%$ C.L. ${ }^{3}$. In any case, from unitary conditions [17] and the experimental value of the vacuum expectation value $v$, one can extract an strong upper limit to the Higgs mass of $M_{h_{S M}} \leq 860 \mathrm{GeV} / \mathrm{c}^{2}$.

However, spin zero fields are radically different from fermions and gauge bosons. The latter are protected from large radiative corrections to their masses thanks to chiral and gauge symmetries, respectively. In the SM there is no mechanism to prevent scalar particles from acquiring large masses through radiative corrections. Therefore, $m_{H}^{2}$ receives enormous quantum corrections from the virtual effects of every particle which couples to the Higgs field (see one-loop diagrams in Fig. 2.5).
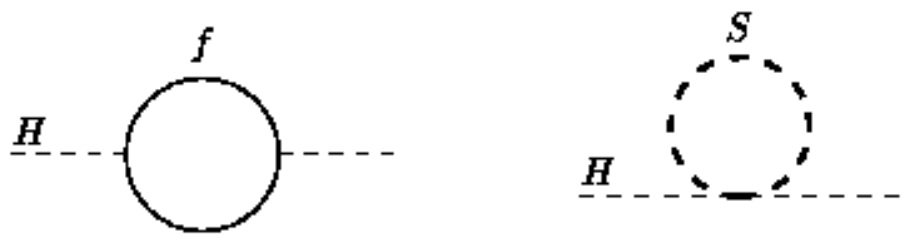

Fig. 2.5: One-loop quantum corrections to $m_{h_{S M}}^{2}$.

Due to these corrections, the Higgs mass would be

$$
m_{h_{S M}}^{2}=\left(m_{h}^{2}\right)_{0}+\Delta M_{H}^{2}
$$

where $\left(m_{h}^{2}\right)_{0}$ is the bare Higgs mass and $\Delta M_{H}^{2}$ is the correction given by

$$
\Delta M_{H}^{2}=-\frac{\lambda_{f}^{2}}{16 \pi^{2}}\left[2 \Lambda^{2}+o\left(m_{f}^{2} \ln \left(\frac{\Lambda}{m_{f}}\right)\right)\right]
$$

where $\lambda_{f}$ is the Yukawa coupling of the fermion $f$ and $\Lambda$ is an energy cutoff which is interpreted as the energy scale at which new physics enters and changes the high-energy behaviour of the theory. If the SM needs to describe nature until the Planck scale, then the quantum correction $\Delta M_{H}^{2}$ is about 30 orders of magnitude larger than the bare Higgs mass square. A cancellation of these corrections at all orders would call for an incredible "fine tunning" which seems very unlikely [18]. This problem is present even if there is no direct coupling between the Standard model Higgs boson and the unknown heavy particles [19].

\footnotetext{
${ }^{3}$ Although last measurements from the top mass from CDF II can slightly change this value.
} 
In a model with spontaneous electroweak symmetry breaking, the problem affects not only to the Higgs mass but also its expectation value and the masses of other particles that get their masses through this mechanism such as the $W, Z$, quarks and charged leptons. This situation has also an analogy with the self-energy corrections on the electron, which is solved by the presence of the positron [20]. Hence, it is unnatural to have all the SM particles masses at the electroweak scale unless the model is somehow cut off and embedded in a richer structure at energies no bigger than the TeV scale.

\subsection{The Supersymmetric extension of the Standard Model}

\subsubsection{Supersymmetry}

Supersymmetry (SUSY) [7][19] is a symmetry which relates masses and couplings of bosons and fermions via spin- $\frac{1}{2}$ charges. In SUSY, particles are combined into superfields and an operator $Q$ generates the transformation of converting fermions to bosons and vice versa:

$$
Q \mid \text { Boson }>=\mid \text { Fermion }>\quad Q \mid \text { Fermion }>=\mid \text { Boson }>
$$

Therefore $Q$ is a complex anticommuting spinor and its hermitian conjugate, $Q^{\dagger}$, is also a symmetry generator. Both of them are fermionic in nature $(S=1 / 2)$ and form a Lie algebra [21], together with the four-momentum and the Lorentz transformation generators. In fact, SUSY is a generalisation of the space-time symmetries of quantum field theory and seems to be the last possible extension of the Lorentz group [22].

In this situation, each chiral fermion $f_{L, R}$ has a scalar partner $\tilde{f}_{L, R}$ and for each massless gauge boson $A_{\mu}$, with helicity states \pm 1 , there is a massless spin $1 / 2$ gaugino partner, with helicity states $\pm \frac{1}{2}$.

\subsubsection{Supersymmetry and the hierarchy problem}

The SM hierarchy problem presented in section 2.2.1 is very elegantly solved when considering the supersymmetric theory [23]. The reason is that every fermion $f$ has a scalar SUSY partner $S$ that couples to the Higgs as well and contributes with a mass correction term of the form:

$$
\Delta M_{H}^{2}=\frac{\lambda_{S}^{2}}{16 \pi^{2}}\left[2 \Lambda^{2}+o\left(m_{S}^{2} \ln \left(\frac{\Lambda}{m_{S}}\right)\right)\right]
$$

Since now $\lambda_{f}=\lambda_{S}$ and Fermi statistics implies an opposite sign with respect to the contribution stated in Eq. (2.47), all the terms have a counter-term that naturally cancel the huge 
corrections. The terms that do not cancel are of the form:

$$
\Delta M_{H}^{2}=\frac{\lambda^{2}}{16 \pi^{2}}\left|m_{S}^{2}-m_{f}^{2}\right|
$$

where some smaller contributions have been omitted. This result leads us to the following "naturalness" argument [24]: since these corrections must not be greater than $m_{h_{S M}}$ in order to avoid too much fine tuning, then

$$
\left|m_{S}^{2}-m_{f}^{2}\right| \lesssim 1 \mathrm{TeV}^{2} .
$$

Hence, one associates $\Lambda \sim 1 \mathrm{TeV}$ as the scale where the SM is no longer valid and must be substituted by its supersymmetric extension. As a benefit, this new theory would be valid all the way up to the Planck scale. In any case, this is only a qualitative argument and does not help predicting exactly whether new particles should appear at $900 \mathrm{GeV}$ or $2 \mathrm{TeV}$.

\subsubsection{Other benefits from the introduction of SUSY}

Besides making a small Higgs mass natural, SUSY has other interesting consequences. One of them is that when SUSY is locally realised it contains among its gauge fields the gravitino. Thus SUSY seems to be a good candidate for a theory of all interactions, or at least to play an important role in any such theory. In addition, Great Unifications Theories (GUT) also provide good motivation for the existence of supersymmetry. One can use the running of the three couplings of the SM, measured at the electroweak scale, and find that, at a certain GUT scale of $10^{15} \mathrm{GeV}$, the couplings almost become the same value [25]. But if one considers SUSY then the couplings are modified in such a way that they become precisely the same value at the GUT scale. This is a strong theoretical motivation for the need of SUSY. However, some people claim that there is nothing special on that [26] provided that other models could do it if they introduce as many parameters as SUSY does.

In addition to gauge coupling unification, SUSY is also a key ingredient for GUT. These theories have interesting predictions such as a small neutrino mass of the order of $m_{v} \approx m_{W}^{2} / m_{G U T} \approx$ $10^{-2} \mathrm{eV} / \mathrm{c}^{2}$ and it can lead to the understanding of the different quark and lepton quantum numbers. But without SUSY the lifetime of the proton would be too small and the prediction for $\sin ^{2} \theta_{W}$ would differ from the experiment [27]. In addition, SUSY has been of greatest interest in string theories since it is the mechanism which provides a coherent and complete framework which avoids negative square masses in some vibrational modes (tachyons) [28].

Furthermore, some SUSY models predict the presence of a lightest supersymmetric particle, which is a candidate for dark matter in the universe, provided that it is neutral, weakly interacting and stable.

As a final remark, recent fits on the electroweak precision observables, such as the effective leptonic weak mixing angle, $\sin ^{2} \theta_{\text {eff }}$, seem to favour supersymmetric models in front of the SM 
alone [29]. This can be seen in Fig. 2.6, where the SM predictions for the $M_{W}$ as a function of $m_{t}$ is being compared with the predictions from the unconstrained Minimal Supersymmetric Standard Model (MSSM), which will be described in the next subsection. The predictions within the two models give rise to two bands with only a relatively small overlap region. The allowed parameter region in the SM arises from varying the only free parameter of the model, the mass of the SM Higgs boson from $M_{h_{S M}}=114 \mathrm{GeV} / \mathrm{c}^{2}$ (upper edge of the band) to $400 \mathrm{GeV} / \mathrm{c}^{2}$ (lower edge of the band). For the MSSM area, SUSY masses close to their experimental limit are assumed for the upper edge, while the MSSM with large masses yields the lower edge of the blue area (dark-shaded). The 68\% C.L. experimental results slightly favours the MSSM over the SM${ }^{4}$.

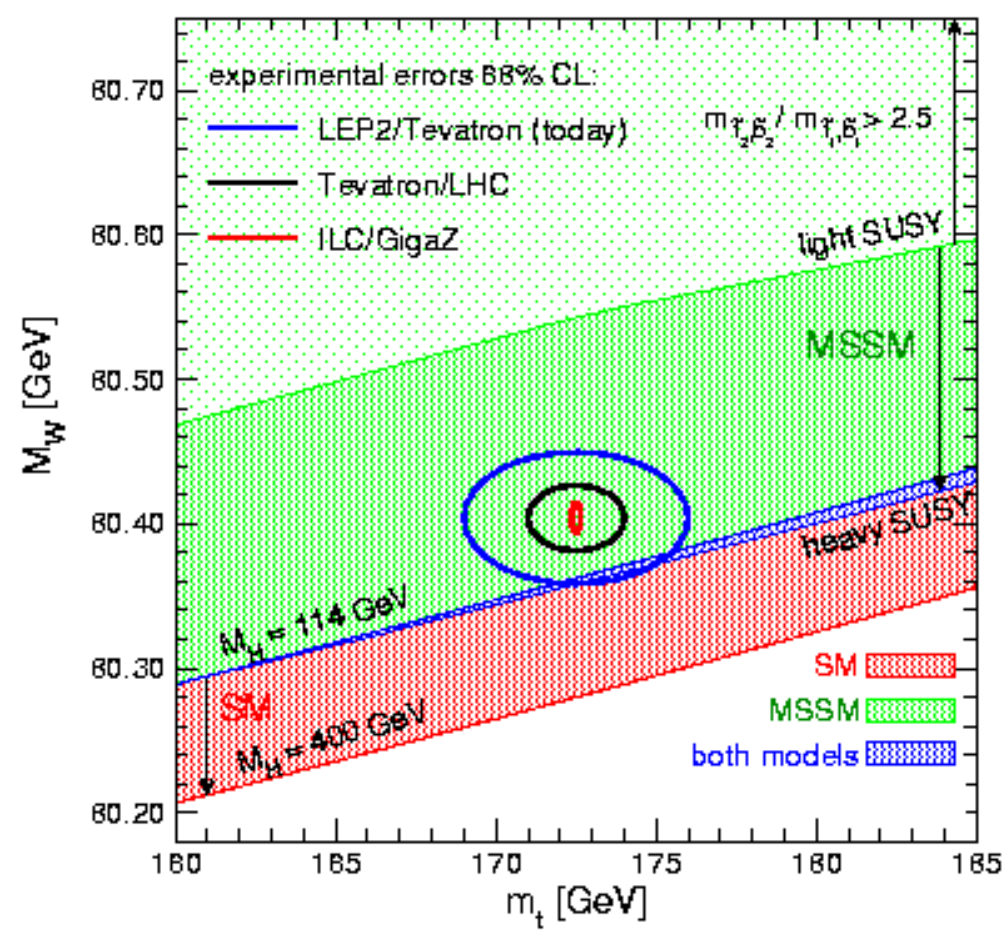

Fig. 2.6: $M_{W}$ as a function of $m_{t}$ as predicted by the SM in red (medium-shaded) and blue (dark-shaded) bands and with the MSSM prediction in green (light-shaded) and blue (dark-shaded) bands. The perspectives for the present and future generation colliders, are also stated.

\subsubsection{The Minimal Supersymmetric Standard Model}

Similarly to the SM construction, that was conceived to be the minimal group viable to explain the electroweak sector, the MSSM [31] is the minimal viable supersymmetric extension of the

\footnotetext{
${ }^{4}$ Last top mass measurements from the Tevatron [30] indicate even a lower mass for the top: $m_{t}=171.4 \pm$ $1.2($ stat $) \pm 1.8($ syst $) \mathrm{GeV} / \mathrm{c}^{2}$.
} 
SM. The MSSM obeys the same $\mathrm{SU}(3)_{C} \otimes \mathrm{SU}(2)_{L} \otimes \mathrm{U}(1)_{\mathrm{Y}}$ gauge symmetries of the Standard Model but doubles the spectrum of new particles since for every particle in the SM, a superpartner is postulated which differs by half a unit of spin. The superpartners are conveniently described by a notation with close correspondance to the SM notation for bosons and fermions. Hence, the superpartners are written with the same letter of their partner but with a tilde over it and the superfields are written with a "hat" superscript. In addition, the bosonic partners of the fermions are denoted starting with an extra "s" (e.g. selectron is the superpartner of the electron) and the fermionic partners of the bosons finish with the suffix "ino" (e.g. gluino is the superpartner of the gluon).

For simplicity and to avoid unnecessary repetitions, consider the case of one generation of quarks, leptons and their superpartners. One can define $\hat{Q}$ as the superfield containing an $\mathrm{SU}(2)_{L}$ doublet of quarks:

$$
Q=\left(\begin{array}{c}
u_{L} \\
d_{L}
\end{array}\right)
$$

and their scalar partners which are also in an $\mathrm{SU}(2)_{L}$ doublet,

$$
\tilde{Q}=\left(\begin{array}{c}
\tilde{u}_{L} \\
\tilde{d}_{L}
\end{array}\right)
$$

In an analogous form, the superfield $\hat{U}^{c}\left(\hat{D}^{c}\right)$ contains the right-handed up (down) anti-quark, $\bar{u}_{R}\left(\bar{d}_{R}\right)$, and its scalar partner, $\tilde{u}_{R}^{*}\left(\tilde{d}_{R}^{*}\right)$. Following the same pattern, leptons are contained in the $S U(2)_{L}$ doublet superfield $\hat{L}$ which contains the left-handed fermions,

$$
L=\left(\begin{array}{c}
\mathrm{v}_{L} \\
e_{L}
\end{array}\right)
$$

and their scalar partners,

$$
\tilde{L}=\left(\begin{array}{c}
\tilde{\mathrm{v}}_{L} \\
\tilde{e}_{L}
\end{array}\right)
$$

Finally, the superfield $\hat{E}^{c}$ contains the right-handed anti-electron, $\bar{e}_{R}$, and its scalar partner, $\tilde{e}_{R}^{*}$.

Similarly, for every gauge boson it exist a Majorana fermion (gaugino). $\hat{G}^{a}$ is defined as a superfield that contains all the gluons, $g^{a}$, and their fermion partners the gluinos, $\tilde{g}^{a} ; \hat{W}_{i}$ contains the $S U(2)_{L}$ gauge bosons, $W_{i}$, and their fermion partners, $\tilde{\omega}_{i}$ (winos); and $\tilde{B}$ contains the $U(1)$ gauge field, $B$, and its fermion partner, $\tilde{b}$ (bino).

In addition, in the MSSM the Higgs sector is enlarged to avoid triangle gauge anomalies [32]. Gauge theories cannot have anomalies and this is simply achieved by requiring that the sum of all 


\begin{tabular}{|c|c|cc|cc|c|}
\hline \multicolumn{2}{|c|}{ Names } & \multicolumn{2}{|c|}{ 2HDM particle } & SUSY partner & $S U(3)_{C}, S U(2)_{L}, U(1)_{Y}$ \\
\hline \hline \multirow{3}{*}{$\begin{array}{c}\text { squarks, quarks } \\
\text { (x 3 families) }\end{array}$} & $\hat{Q}$ & $\left(u_{L} d_{L}\right)$ & $\frac{1}{2}$ & $\left(\tilde{u}_{L} \tilde{d}_{L}\right)$ & 0 & $\left(3,2, \frac{1}{3}\right)$ \\
\cline { 2 - 7 } & $\hat{U}$ & $u_{R}^{\dagger}$ & $\frac{1}{2}$ & $\tilde{u}_{R}^{*}$ & 0 & $\left(\overline{3}, 1,-\frac{4}{3}\right)$ \\
\cline { 2 - 7 } & $\hat{D}$ & $d_{R}^{\dagger}$ & $\frac{1}{2}$ & $\tilde{d}_{R}^{*}$ & 0 & $\left(\overline{3}, 1, \frac{2}{3}\right)$ \\
\hline \multirow{2}{*}{$\begin{array}{c}\text { sleptons, leptons } \\
\text { (x 3 families) }\end{array}$} & $\hat{L}$ & $\left(\mathrm{v} e_{L}\right)$ & $\frac{1}{2}$ & $\left(\tilde{v} \tilde{e}_{L}\right)$ & 0 & $(1,2,-1)$ \\
\cline { 2 - 7 } & $\hat{E}$ & $e_{R}^{\dagger}$ & $\frac{1}{2}$ & $\tilde{e}_{R}^{*}$ & 0 & $(1,1,2)$ \\
\hline \hline \multirow{2}{*}{$\begin{array}{c}\text { Electroweak bosons } \\
\text { Strong bosons }\end{array}$} & $\hat{W}$ & $W^{1} W^{2} W^{3}$ & 1 & $\tilde{W}^{1} \tilde{W}^{2} \tilde{W}^{3}$ & $\frac{1}{2}$ & $(1,3,0)$ \\
\cline { 2 - 7 } & $\hat{B}$ & $B$ & 1 & $\tilde{B}_{a}$ & $\frac{1}{2}$ & $(1,1,0)$ \\
\hline \hline \multirow{2}{*}{ Higgs, higgsinos } & $\hat{G}_{a}$ & $g_{a}$ & 1 & $\tilde{g}_{a}$ & $\frac{1}{2}$ & $(8,1,0)$ \\
\cline { 2 - 7 } & $\hat{H}_{u}$ & $\left(H_{u}^{+} H_{u}^{0}\right)$ & 0 & $\left(\tilde{H}_{u}^{+} \tilde{H}_{u}^{0}\right)$ & $\frac{1}{2}$ & $(1,2,1)$ \\
\hline \hline
\end{tabular}

Tab. 2.3: Superfields and particle content of the MSSM. Symbols for each of the chiral supermultiplets as a whole are indicated in the second column.

fermion charges vanishes. The Higgs scalar doublet acquires a SUSY partner which is an $\mathrm{SU}(2)_{L}$ doublet of Majorana fermion fields, $\tilde{h}_{1}$ (Higgsinos), which will contribute to the triangle $\operatorname{SU}(2)_{L}$ and $\mathrm{U}(1)_{Y}$ gauge anomalies. Since fermions in SM have exactly the right quantum numbers to cancel these anomalies, it follows that the contribution from the fermionic partner of the Higgs doublet remains uncancelled. The easiest solution is to require a second Higgs doublet with precisely the opposite $\mathrm{U}(1)_{Y}$ quantum number than the first Higgs doublet. Furthermore, in the SM the Higgs doublet (the complex conjugate of the doublet) can couple to the $T_{3}=+\frac{1}{2}\left(T_{3}=-\frac{1}{2}\right)$ fermions and give mass to all the spectrum of fermions. But, in a supersymmetric theory, any doublet can give mass either to a $T_{3}=+\frac{1}{2}$ or a $T_{3}=-\frac{1}{2}$ fermion but not to both. Thus, two Higgs doublets are needed in order to generate both up-like and down-like quark masses. As result, one could think of the SM becoming a two Higgs doublet model (2HDM) [33] prior to introduce the supersymmetric sector. In Tab. 2.3 the spectrum of the MSSM fields is summarised.

With two SU(2) doublets, the theory has eight real scalar fields and three massless gauge bosons, which accounts for fourteen degrees of freedom. After SUSY breaking, the three gauge bosons acquire masses (nine degrees of freedom), which means that there should exist five spinzero Higgs fields in the spectrum: three neutral scalars $(h, H, A)$ and two charged pairs $\left(H^{+}\right.$, $\left.H^{-}\right)$.

The parameters of the supersymmetry-conserving sector consist of:

- Gauge couplings: $g_{s}, g$ and $g^{\prime}$, corresponding to the Standard Model gauge group $\mathrm{SU}(3)_{C} \otimes$ $\mathrm{SU}(2)_{L} \otimes \mathrm{U}(1)_{\mathrm{Y}}$, respectively.

- Higgs mass parameter, $\mu$. 
- Higgs-fermion Yukawa coupling constants: $\lambda_{u}, \lambda_{d}$, and $\lambda_{e}$, corresponding to the coupling of quarks or leptons and their superpartners to the Higgs bosons and higgsinos.

The supersymmetry-breaking sector contains the following set of parameters:

- Gaugino Majorana masses $M_{3}, M_{2}$ and $M_{1}$, associated with the $\mathrm{SU}(3)_{C}, \mathrm{SU}(2)_{L}$ and $\mathrm{U}(1)_{\mathrm{Y}}$ subgroups, respectively. These masses may be connected in some cases as will be seen later.

- Five scalar squared-mass parameters for the squarks and sleptons: $M_{\tilde{Q}}^{2}, M_{\tilde{U}}^{2}, M_{\tilde{D}}^{2}, M_{\tilde{L}}^{2}$ and $M_{\tilde{E}}^{2}$, corresponding to the five electroweak gauge multiplets.

- Trilinear interaction terms of the form Higgs-squark-squark and Higgs-slepton-slepton, with coefficients $A_{u}, A_{d}$ and $A_{e}$.

- Three scalar Higgs squared-mass parameters, two of which $\left(m_{1}^{2}\right.$ and $\left.m_{2}^{2}\right)$ contribute to the diagonal Higgs squared-masses and a third which corresponds to the off-diagonal terms $m_{12}^{2} \equiv \mu B$. These three parameters can be re-expressed in terms of the two Higgs vacuum expectation values $\left(v_{d}=<H_{d}^{0}>\text { and } v_{u}=\left\langle H_{u}^{0}\right\rangle\right)^{5}$, usually taken through the ratio

$$
\tan \beta \equiv \frac{v_{u}}{v_{d}},
$$

and one physical Higgs mass ${ }^{6}$.

The gluino is the color octet Majorana (there is no distinct antigluon) fermion partner of the gluon. It has 16 degrees of freedom since there are 8 massless gluons ( 2 spin degrees of freedom, each). The supersymmetric partners of the electroweak gauge and Higgs bosons (gauginos and higgsinos) can mix. As a result, the physical mass eigenstates are model-dependent linear combinations of these states, called charginos and neutralinos, which are obtained by diagonalising the corresponding mass matrices. There are two charginos $\left(\tilde{\chi}_{i}^{ \pm}\right)$and four neutralinos $\left(\tilde{\chi}_{i}^{0}\right)$, which are by convention ordered in masses ( $\tilde{\chi}_{1}^{ \pm}$is the lowest chargino and $\tilde{\chi}_{1}^{0}$ is the lowest neutralino). Depending whether the chargino or neutralino eigenstate approximates a particular gaugino or higgsino state, they can become more photino-like, bino-like... and result in strinkingly different phenomenology.

The supersymmetric partners of the quarks and leptons are spin-zero bosons and the resulting squarks and sleptons can also mix their left- and right-handed components yielding the mass eigenstates (denoted by the indices 1,2 instead of $L, R$ ). This mixing is proportional to the mass of the SM partner quark or lepton and to $\tan \beta$. Thus, the mixing can lead to an important splitting

\footnotetext{
${ }^{5}$ Notation $v_{u}\left(v_{d}\right)$ is used to distinguish vacuum expectation values of the Higgs field which couples exclusively to up-type (down-type) quarks.

${ }^{6}$ Note that $v_{d}^{2}+v_{u}^{2}=4 M_{W}^{2} / g^{2}=\left(246 \mathrm{GeV} / \mathrm{c}^{2}\right)^{2}$ is fixed by the $W$ mass and the gauge coupling, but $\tan \beta$ is a free parameter of the model.
} 


\begin{tabular}{|ll|c||ll|c|}
\hline \multicolumn{2}{|l|}{ 2HDM particle } & spin & SUSY particle & spin \\
\hline \hline quarks: & $q$ & $\frac{1}{2}$ & squarks: & $\tilde{q}_{1}, \tilde{q}_{2}$ & 0 \\
leptons: & $l$ & $\frac{1}{2}$ & sleptons: & $\tilde{l}_{1}, \tilde{l}_{2}$ & 0 \\
gluons: & $g_{a}$ & 1 & gluinos: & $\tilde{g}_{a}$ & $\frac{1}{2}$ \\
gauge bosons: & $W^{ \pm}, Z^{0}, \gamma$ & 1 & neutralinos: $\tilde{\chi_{1}^{0}}, \tilde{\chi_{2}^{0}}, \tilde{\chi_{3}^{0}}, \tilde{\chi_{4}^{0}}$ & $\frac{1}{2}$ \\
Higgs bosons: & $h^{0}, H^{0}, A^{0}, H^{ \pm}$ & 0 & charginos: $\tilde{\chi_{1}^{ \pm}}, \tilde{\chi_{2}^{ \pm}}$ & $\frac{1}{2}$ \\
\hline
\end{tabular}

Tab. 2.4: The particle content of the MSSM.

in the mass spectrum of heavy squarks, specially at large $\tan \beta$. In contrast, the first two families can be considered degenerate in mass. All physical particles of the MSSM are given in Tab. 2.4.

\subsubsection{MSSM lagrangian and R-parity}

The MSSM lagrangian is constructed using the already defined particle content and following an analogy with the ${ }_{L_{S M}}$. Following a similar notation as in the SM, the kinetic term of the lagrangian can be written as:

$$
\begin{aligned}
\mathcal{L}_{K E} & =\sum_{i}\left\{\left(D_{\mu} S_{i}\right)^{\dagger}\left(D^{\mu} S_{i}\right)+\frac{i}{2} \bar{\psi}_{i} \gamma^{\mu} D_{\mu} \psi_{i}\right\} \\
& +\sum_{A}\left\{-\frac{1}{4} F_{\mu v}^{A} F^{\mu v A}+\frac{i}{2} \bar{\lambda}_{A} D \lambda_{A}\right\} .
\end{aligned}
$$

Here, $S_{i}\left(\psi_{i}\right)$ is the scalar (fermion) component of the $i^{t h}$ chiral superfield, $D$ is the $S U(3) \times$ $S U(2)_{L} \times U(1)$ gauge invariant derivative, $F_{\mu v}^{A}$ is the Yang-Mills gauge field and $\lambda_{A}$ is the gaugino superpartner of the corresponding gauge boson. It is worth noticing that the $\sum_{i}$ is a sum over all fermion fields of the SM, the scalar partners and the 2 Higgs doublets with their fermion partners. On the other hand, $\sum_{A}$ is over the $S U(3)_{c}, S U(2)_{L}$ and $U(1)_{Y}$ gauge fields with their fermion partners, the gauginos.

The interactions between bosons and fermions are described by:

$$
\begin{aligned}
\mathcal{L}_{\text {int }}= & -\sqrt{2} \sum_{i, A} g_{A}\left[S_{i}^{*} T^{A} \bar{\psi}_{i L} \lambda_{A}+\text { h.c. }\right] \\
& -\frac{1}{2} \sum_{A}\left(\sum_{i} g_{A} S_{i}^{*} T^{A} S_{i}\right)^{2}
\end{aligned}
$$

where $\psi_{L} \equiv \frac{1}{2}\left(1-\gamma_{5}\right) \psi, T^{A}$ is the matrix of the group generators and $g_{A}$ the gauge coupling constants. It can be seen that there are no adjustable parameter, hence, all interaction strengths are completely fixed in terms of SM coupling constants. 
Once the superfields and the gauge symmetries are chosen, the only freedom in constructing $\mathcal{L}_{M S S M}$ is contained in a function called superpotential, $w$. This is an analytic form of the chiral superfields, $\hat{S}$, that has the form:

$$
w=\varepsilon_{i j} \mu \hat{H}_{u}^{i} \hat{H}_{d}^{j}+\varepsilon_{i j}\left[\lambda_{L} \hat{H}_{d}^{i} \overline{\hat{L}}^{j} \overline{\hat{E}}+\lambda_{D} \hat{H}_{d}^{i} \hat{Q} \hat{\bar{D}}+\lambda_{U} \hat{H}_{u}^{j} \hat{Q}^{i} \hat{\bar{U}}\right]+W_{R P}
$$

where $i$ and $j$ are $S U(2)_{L}$ doublet indices and $\varepsilon_{i j}=-\varepsilon j i$ (with $\varepsilon_{12}=1$ ) contracts the $S U(2)_{L}$ doublet fields. No derivative interactions are allowed in order that $w$ be an analytical function. The term $\mu \hat{H}_{u}^{i} \hat{H}_{d}^{j}$ gives mass terms for the Higgs bosons and so $\mu$ is often called the Higgs mass parameter. The terms in the square brackets proportional to $\lambda_{L}, \lambda_{D}$ and $\lambda_{U}$ give the usual Yukawa interactions of the fermions with the Higgs bosons. Hence, unlike the SM case, these coefficients are determined in terms of the fermion masses and the vacuum expectation values of the neutral members of the scalar components, and are not arbitrary couplings.

In the most general superpotential one can add more terms which are grouped under $W_{R P}$ in Eq. (2.59). These terms are of the form:

$$
W_{R P}=\lambda_{\alpha \beta \gamma} \hat{L}^{\alpha} \hat{L}^{\beta} \overline{\hat{E}}^{\gamma}+\lambda_{\alpha \beta \gamma}^{\prime} \hat{L}^{\alpha} \hat{Q}^{\beta} \overline{\hat{D}}^{\gamma}+\lambda_{\alpha \beta \gamma}^{\prime \prime} \overline{\hat{U}}^{\alpha} \overline{\hat{D}}^{\beta} \overline{\hat{D}}^{\gamma}+\mu^{\prime} \hat{L} \hat{H}
$$

where the indices $\alpha, \beta$ and $\gamma$ label the 3 generations of quarks and leptons. These terms constitute a problem in the sense that the first two contribute to lepton number violation interactions and the third one to baryon number violation interactions ${ }^{7}$. The combination of lepton and baryon violation terms can contribute to the proton decay at tree level through the exchange of the scalar partner of the down quark. Since this process is experimentally restricted [35][36] it put into question the validity of the model. One solution is to assume that the parameters are small enough to avoid experimental limits. Even this is certainly allowed experimentally, this would imply the introduction of an artificial tuning. The other solution is to introduce a new symmetry called R-parity [37]. R-parity $\left(R_{p}\right)$ is a multiplicative quantum number defined as:

$$
R=(-1)^{3(B-L)+2 s},
$$

where $B$ and $L$ are the baryon and lepton quantum numbers and $s$ is the spin of the particle. Thus, all SM particles have $R_{p}=+1$ while their SUSY partners have $R_{p}=-1$.

The assumption of such a symmetry prevents lepton and baryon number violating terms but has also dramatic phenomenological consequences: there can be no mixing between the sparticles and the $R_{P}=1$ particles, SUSY particles can only be pair-produced in the collisions of SM particles and a SUSY particle would undergo a chain of decays until the lightest SUSY particle (LSP) is produced. Then, this LSP cannot decay further and constitutes a cold dark matter candidate ${ }^{8}$.

\footnotetext{
${ }^{7}$ The fourth term can be ignored since one can implement a rotation in the lepton field $\hat{L}$ such that this term vanishes [34].

${ }^{8}$ Due to cosmological constraints, a cold dark matter candidate need to be stable and neutral [38][39].
} 


\subsubsection{SUSY breaking}

At this point, the MSSM lagrangian does not provide mass terms for all the particles (fermions, scalars, gauge fields). If supersymmetry was an exact symmetry, squarks and quarks would have equal masses and gluinos would be massless. Since this is not the case in nature, at low energies supersymmetry must be a broken symmetry and new SUSY-breaking terms need to be introduced in the lagrangian. To prevent dangerous quadratic divergences, only a certain subset of supersymmetry-breaking terms are present in the theory and their couplings are denoted as soft parameters. Then, the so-called soft lagrangian which breaks SUSY is (first generation only):

$$
\begin{aligned}
-\mathcal{L}_{\text {soft }} & =\frac{1}{2}\left[M_{3} \hat{g} \hat{g}+M_{2} \hat{W} \hat{W}+M_{1} \hat{B} \hat{B}\right] \\
& +\varepsilon_{\alpha \beta}\left[-b H_{d}^{\alpha} H_{u}^{\beta}-H_{u}^{\alpha} \hat{Q}_{i}^{\beta} \hat{A}_{u_{i j}} \overline{\hat{U}}_{j}+H_{d}^{\alpha} \hat{Q}_{i}^{\beta} \hat{A}_{d_{i j}} \overline{\hat{D}}_{j}+H_{d}^{\alpha} \hat{L}_{i}^{\beta} \hat{A}_{e_{i j}} \overline{\hat{E}}_{j}+\text { h.c. }\right] \\
& +m_{H_{d}}^{2}\left|H_{d}\right|^{2}+m_{H_{u}}^{2}\left|H_{u}\right|^{2}+\hat{Q}_{i}^{\alpha} m_{Q_{i j}}^{2} \hat{Q}_{j}^{\alpha *} \\
& +\hat{L}_{i}^{\alpha} m_{L_{i j}}^{2} \hat{L}_{j}^{\alpha *}+\overline{\hat{U}}_{i}^{*} m_{U_{i j}}^{2} \overline{\hat{U}}_{j}+\overline{\hat{D}}_{i}^{*} m_{D_{i j}}^{2} \overline{\hat{D}}_{j}+\overline{\hat{E}}_{i}^{*} m_{E_{i j}}^{2} \overline{\hat{E}}_{j},
\end{aligned}
$$

where $i$ and $j$ are the $S U(2)_{L}$ doublet indices. This Lagrangian has arbitrary masses for the scalars and gauginos and also arbitrary bi-linear and tri-linear mixing terms. The scalar and gaugino mass terms have the desired effect of breaking the mass degeneracy between the particles and their SUSY partners. The tri-linear A terms affect primarily the particles of the third generation. The $\mu B$ term mixes the scalar components of the two Higgs doublets. In the most general case, all of the mass and interaction terms of Eq. (2.62) are matrices involving all three generators. However, the origin of all these terms is left unspecified. How supersymmetry breaking is transmitted to the superpartners is encoded in the parameters of $L_{s o f t}$. All of the quantities in $L_{s o f t}$ receive radiative corrections and thus are scale-dependent, satisfying known Renormalisation Group Equations (RGEs).

For phenomenological purposes, the MSSM lagrangian is simply a low energy effective lagrangian with a number of input parameters. The fact that except for the assumption of the presence of supersymmetric particles, $R_{p}$, and gauge and Poincaré invariance, nothing else has been assumed, makes from the MSSM a very simple framework but one needs to introduce plenty of free input parameters. MSSM includes at least 105 new parameters that added to the 19 parameters of the SM, the model has 124 parameters to be determined ${ }^{9}$. While often only subsets of these parameters are relevant for particular experimental processes and there exist some phenomenological constraints in these parameters, the number is too large for practical purposes to carry out phenomenological analyses in full generality.

However, unlike in the SM case, now there is the possibility to stablish a top-down approach by which the MSSM parameters are predicted within the context of an underlying theory, often

\footnotetext{
${ }^{9}$ For this particular reason, sometimes it is referred to as MSSM-124.
} 
as functions of fewer basic parameters. The basic question to be addressed is how to understand the explicit soft supersymmetry breaking encoded in the $L_{\text {soft }}$ parameters as the result of spontaneous supersymmetry breaking in a more fundamental theory. Since this is not known, different models have been constructed as an attempt to find an answer for this question. Since $\mathrm{TeV}$ scale supersymmetry breaking models have reported negative results [40], other models which assume that the theory can be splitted into at least two sectors have been considered. These two sectors have no direct renormalizable couplings between them and they are divided into observable or visible sector, which contains the SM fields and their superpartners, and the hidden sector, in which supersymmetry is spontaneously broken by a dynamical mechanism.

Within this framework, SUSY breaking is communicated from the hidden sector where it originates to the observable sector via suppressed interactions involving a third set of fields: the mediator or messenger fields. This hidden sector implies that the fundamental scale of supersymmetry breaking $\mu_{s}$ is hierarchically larger than the TeV scale. Depending on the model this $\mu_{s}$ can be postulated to be at the GUT scale, Majorana neutrino mass scale or in extra-dimensional braneworlds. Therefore, different models account for specific mechanisms on how supersymmetry breaking is mediated between the hidden and observable sectors and involve specific energy scales at which the soft terms are generated. These values are then used to compute the corresponding values at observable energy scales, all predicted at the TeV scale by the models, using the scale dependence of the $\mathcal{L}_{\text {soft }}$ parameters as dictated by their RGEs.

\subsubsection{Constraining the MSSM: mSUGRA model}

The observation that the measured coupling constants tend to meet at a point when evolved to high energy scales inspired many SUSY GUT models. In these models, the value of the couplings at the GUT scale $M_{X} \sim 10^{16} \mathrm{GeV} / \mathrm{c}^{2}$ plays a central role [34]:

$$
\sqrt{\frac{5}{3}} g_{1}\left(M_{X}\right)=g_{2}\left(M_{X}\right)=g_{s}\left(M_{X}\right) \equiv g^{*} .
$$

Since gravitational interactions are shared by all the particles, it is quite natural to imagine gravity to be the only interaction shared by both the hidden and the observable sector. Furthermore, at some point gravity must be present in particle field theory if a comprehensive description of nature is desired. Here, supergravity would be the responsible of promoting global supersymmetry to local supersymmetry. This is what inspired the mSUGRA model [41].

In this model, along with the coupling constants, the following set of assumptions emerges:

1. Common gaugino mass $m_{1 / 2}$ : the gaugino mass terms, $M_{i}$, are assumed to unify:

$$
M_{i}\left(M_{X}\right) \equiv m_{1 / 2}
$$


2. Common scalar mass $m_{0}$. The soft SUSY-breaking scalar mass terms contributing to the squark, slepton and Higgs boson masses are equal to $m_{0}$ at $M_{X}$ :

$$
\begin{aligned}
m_{\hat{Q}^{2}}\left(M_{X}\right) & =m_{\hat{\hat{u}}}^{2}\left(M_{X}\right)=\cdots \\
& =m_{H_{d}}^{2}\left(M_{X}\right)=m_{H_{u}}^{2}\left(M_{X}\right) \equiv m_{0}^{2} .
\end{aligned}
$$

3. Common trilinear scalar coupling $A_{0}$. The soft trilinear SUSY-breaking terms are all equal to $A_{0}$ at $M_{X}$,

$$
A_{t}\left(M_{X}\right)=A_{b}\left(M_{X}\right)=A_{\tau}\left(M_{X}\right)=\cdots \equiv A_{0} .
$$

Through RGE's the gaugino masses in Eq. (2.64) scale in the same way as the corresponding coupling constants:

$$
M_{i}\left(M_{W}\right)=m_{1 / 2} \frac{g_{i}^{2}\left(M_{W}\right)}{g^{* 2}}
$$

yielding,

$$
\begin{aligned}
& M_{2}=\frac{g^{2}}{g_{s}^{2}} M_{3} \approx 0.3 m_{\tilde{g}} \\
& M_{1}=\frac{5}{3} \frac{g^{\prime 2}}{g^{2}} M_{2}=\frac{5}{3} \tan ^{2} \theta_{W} M_{2} \approx 0.5 M_{2},
\end{aligned}
$$

where every term is evaluated at $M_{W}$ scale and $m_{\tilde{g}}$ is the gluino mass.

The gluino mass is therefore always the heaviest of the gaugino masses. Assuming the relations Eq. (2.64) and Eq. (2.65) in conjunction with SUSY and the gauge structure, leads to the following expressions for the masses of the sfermions (except for the third generation) at the electroweak scale [42]:

$$
\begin{aligned}
m_{\tilde{f}_{L, R}}^{2} & =m_{f}^{2}+m_{0}^{2}+b_{\tilde{f}_{L, R}} m_{1 / 2}^{2} \\
& \pm m_{Z}^{2} \cos 2 \beta\left[T_{3}^{\tilde{f}_{L, R}}-Q_{\tilde{f}_{L, R}} \sin ^{2} \theta_{W}\right]
\end{aligned}
$$

where $\tilde{f}_{L, R}$ is the corresponding left (right) sfermion, $T_{3}^{\tilde{f}_{L, R}}$ and $Q_{\tilde{f}_{L, R}}$ are the third component of the weak isospin and the electric charge of the corresponding fermion $f$, and the coefficients $b$ are derived from the RGE's and can take different values. In particular, $b \approx 6$ for squarks, $\approx 0.5$ for left sleptons and $\approx 0.15$ for right sleptons. Thus, the squarks are heavier than the sleptons, which is not surprising provided that the squarks have strong interactions in addition to electroweak. More concretely, the mass parameters of the first two generations are roughly degenerate while for the third generation masses are typically reduced by a factor of $1-3$. However, the concrete spectrum can vary depending on the $\tan \beta$ value [43]. An approximate view of the mass spectra obtained via the RGEs can be seen in Fig. 2.7. 


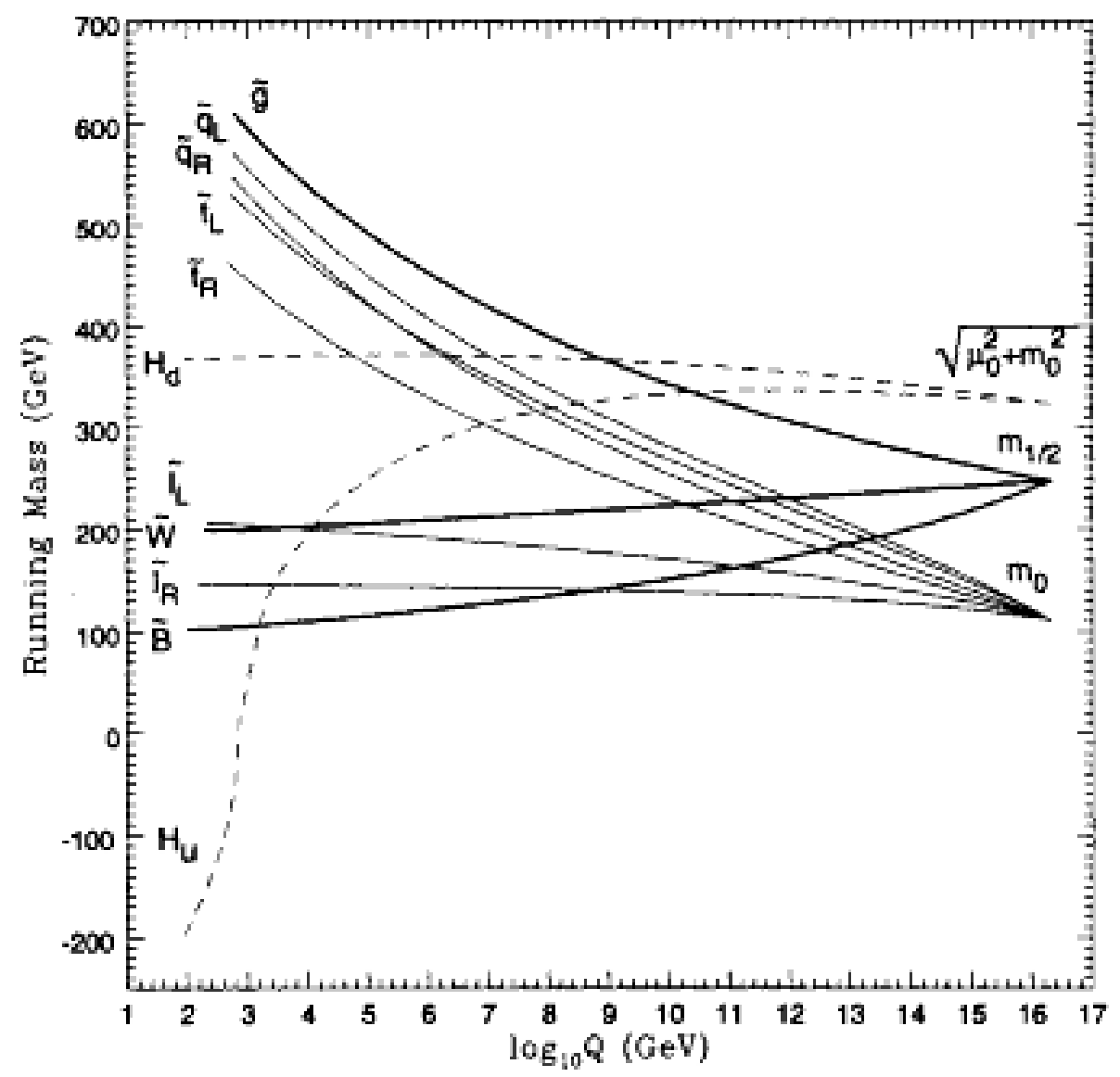

Fig. 2.7: The running of the sparticle masses from the GUT scale to the electroweak scale, for a sample set of input parameters. The bold lines represent the three soft gaugino masses $m_{\tilde{g}}, M_{2}$ (labeled $\tilde{W}$ ) and $M_{1}$ (labeled $\tilde{B}$ ). The light solid lines are the squark $\left(\tilde{q}_{L}, \tilde{q}_{R}, \tilde{t}_{L}, \tilde{t}_{R}\right)$ and the slepton $\left(\tilde{l}_{L}, \tilde{l}_{R}\right)$ soft masses. Finally the dashed lines represent the soft Higgs boson masses labeled by $H_{d}$ and $H_{u}$.

Since the supersymmetry is broken via gravitational interaction, a new massless Goldstone particle (the Goldstino) need to be present. This new particle will be eaten by the gravitino (the spin $3 / 2$ partner of the spin 2 graviton), such that it becomes massive, determining the SUSY breaking scale, $\mu_{S}$ :

$$
m_{\tilde{G}} \sim \frac{\mu_{S}^{2}}{M_{P l}}
$$

Therefore, in mSUGRA model the hidden sector is postulated at the Planck mass and, in order to obtain the new SUSY masses at the desired TeV scale to prevent Higgs mass divergence and to obtain coupling unification at the GUT scale, the SUSY breaking scale (following Eq. (2.70)) should be around $10^{11}-10^{12} \mathrm{GeV}$. The fact that the gravitino mass is of the order of the $\mathrm{TeV}$ is 
a prediction from the mSUGRA model ${ }^{10}$.

With the assumptions Eq. (2.64) - 2.66, the SUSY sector in mSUGRA is completely described by 5 input parameters at the GUT scale [46]: $m_{0}, m_{1 / 2}, A_{0}, \mu$ and $B$, where $\mu$ is the Higgs mass parameter and $B$ the Higgs mixing parameter. The requirement that the $\mathrm{Z}$ boson obtain its measured value when the parameters are evaluated at low energy can be used to restrict $|\mu B|$, leaving the sign of $\mu$ as a free parameter. In addition one can also change the $B$ parameter for $\tan \beta$, leaving the usual parameters of the model:

$$
m_{0}, m_{1 / 2}, A_{0}, \tan \beta, \operatorname{sign}(\mu) .
$$

This model is a simplistic scenario that serves as a good benchmark model since it is extremely predictive as the entire low energy spectrum is predicted in terms of few input parameters. Of course, one needs to bear in mind that changing the input parameters at $M_{X}$ (for example assuming non-universal scalar masses) changes the phenomenology at the weak scale. Therefore one should always perform experimental analyses in view of taking the model as a reference and focus as much as possible on model independent approaches.

\subsection{Squarks and Gluinos}

From all variety of particles that the new MSSM framework introduces, two types of them are of special relevance for this study: squarks and gluinos.

Squarks are the spin-0 boson superpartners of the left- and right-handed quarks. These particles are part of the Supersymmetric QCD (SUSY-QCD) framework, which is based on the coloured particles of the MSSM. The massive states are a mixture of the chiral states, with different contributions defined by the set of RGEs that run in the mSUGRA framework. This mixture is not particularly significant in the first two generations, which can be considered degenerate in mass. However, this is not the case for the stop (due to large top mass) and the sbottom at large $\tan \beta$.

In RGEs, squark mass parameters have a stronger dependence on the common gaugino mass $M_{1 / 2}$ because of colour. For the squarks first and second generation, the left- and right-handed soft SUSY-breaking parameters at electroweak scale are given approximately by:

$$
m_{\tilde{Q}_{1,2}}^{2} \approx m_{0}^{2}+6.3 m_{1 / 2}^{2} \quad ; \quad m_{\tilde{\tilde{u}}_{1,2}}^{2} \approx m_{\tilde{\tilde{d}}_{1,2}}^{2} \approx m_{0}^{2}+5.8 m_{1 / 2}^{2} .
$$

\footnotetext{
${ }^{10}$ The CMSSM (Constrained MSSM) [45] is a model very similar to mSUGRA but that allows for slightly more flexibility like allowing the Higgs sector to be independent of the sfermion sector (while still requiring unification for $m_{0}$ and $m_{1 / 2}$ for the rest of the spectrum). In fact, mSUGRA is the case where SUSY breaking is gravity-mediated in a minimal supergravity scenario. In particular, the relation between the gravitino mass and $m_{0}$ is not necessarily fulfilled in the CMSSM.
} 
In general, squarks are heavier than the sleptons and the lightest neutralino and chargino. A detailed analysis of the SUSY mass spectra at the weak scale can be found in Reference [47] and references therein.

The gluino is the colour octet fermion and it cannot mix with any other particle in the MSSM. In mSUGRA the gluino mass parameter $M_{3}$ is related to the bino and wino masses as shown in the previous chapter:

$$
M_{3}: M_{2}: M_{1} \approx 3.3: 1: 0.5 \text {. }
$$

Therefore, the gluino should be much heavier than the lightest neutralino or chargino. Radiative corrections to the gluino mass can be rather large due to the strong interaction with all squarkquark pairs and its colour octet nature.

A general prediction for mSUGRA is that

$$
m_{\tilde{Q}} \geq 0.85 m_{\tilde{g}},
$$

which holds for the five lightest squarks and small or moderate $\tan \beta$.

\subsection{Squark and Gluino Production Processes}

The hadroproduction of squarks and gluinos at the Tevatron in an $R_{p}$ conserving scenario proceeds through the following partonic reactions:

$$
\begin{array}{ll}
\tilde{q} \overline{\tilde{q}} \text { production: } & q_{i}+\bar{q}_{j} \longrightarrow \tilde{q}_{k}+\overline{\tilde{q}}_{l} \\
& g+g \longrightarrow \tilde{q}_{i}+\overline{\tilde{q}}_{i} \\
\tilde{q} \tilde{q} \text { production: } & q_{i}+q_{j} \longrightarrow \tilde{q}_{i}+\tilde{q}_{j} \text { and c.c. } \\
\tilde{g} \tilde{g} \text { production: } & q_{i}+\bar{q}_{i} \longrightarrow \tilde{g}+\tilde{g} \\
& g+g \longrightarrow \tilde{g}+\tilde{g} \\
\tilde{q} \tilde{g} \text { production: } & q_{i}+g \longrightarrow \tilde{q}_{i}+\tilde{g} \text { and } c . c .
\end{array}
$$

Here, the chiralities of the squarks are not noted explicitly, $\tilde{q}=\left(\tilde{q}_{L}, \tilde{q}_{R}\right)$ and the indices $i-l$ indicate the flavours of the quarks and squarks involved. Charge-conjugate processes (c.c.) are understood for $\tilde{q} \tilde{q}$ and $\tilde{q} \tilde{g}$ production. The corresponding Feynman diagrams are displayed in Fig. 2.8 .

The relative yields of $\overline{\tilde{q} q}, \tilde{q} \tilde{q}, \tilde{g} \tilde{g}$ and $\tilde{q} \tilde{g}$ final states at the Tevatron are shown for a set of mass parameters in Fig. 2.9. They depend strongly on the relative mass difference between the squarks and gluinos. If squarks are lighter than gluinos, the valence partons give the dominant yield of 
squark-antisquark or squark-squark pairs. If the gluinos are the lightest of the two species, their production is the most copious ${ }^{11}$.

The cross sections for the production of squarks and gluinos in hadron collisions were calculated at the Born level in 1992 [49]. In order to reduce the dependence of the cross section on spurious parameters, the predictions were improved to next-to-leading order (NLO) [50]. These predictions increase the production cross sections by a factor of two, approximately, and have a renormalisation scale dependence of $40 \%-50 \%$.

\subsection{Squark and Gluino Decays}

In a mSUGRA scenario with $R_{p}$ conservation, signatures produced by sparticles in the detector are typically related to $\mathbb{E}_{T}$ due to the presence of the LSP (the lightest neutralino, $\tilde{\chi}_{1}^{0}$ ) usually produced after a chain of successive decays of different complexity. The preferred decay modes for squarks are:

$$
\tilde{q} \rightarrow q \tilde{g} \quad \tilde{q} \rightarrow q \tilde{\chi}_{i}^{0} \quad \tilde{q} \rightarrow q^{\prime} \tilde{\chi}_{i}^{ \pm} .
$$

The preference for one decay or the other depend on the available phase space. In the case of the gluino, since it has only strong interactions, its decay proceeds through on-shell or virtual squarks:

$$
\tilde{g} \rightarrow \bar{q} \tilde{q}_{L, R}\left(\tilde{g} \rightarrow q \overline{\tilde{q}}_{L, R}\right) \quad \tilde{g} \rightarrow q q^{\prime} \tilde{\chi}_{i}^{0} \quad \tilde{g} \rightarrow q q^{\prime} \tilde{\chi}_{i}^{ \pm} .
$$

The decay of charginos and neutralinos is quite complex since there are several possibilities and the final-state branching fractions are small and quite sensitive to the model. But for an inclusive search, one can expect at least one (two) jets and missing transverse energy for every squark (gluino) produced. In addition, extra jets from initial and final state radiation can be present as well as some leptons coming from chargino/neutralino decays.

\subsection{Experimental constraints: Run I limits}

There are several direct and indirect experimental constraints to different SUSY processes explained elsewhere [7]. In particular, $\mathrm{CDF}$ and $\mathrm{D} \varnothing$ collaborations have searched inclusively for squark-gluino processes during Run I data taking period. The studies [51] searched with $84 \mathrm{pb}^{-1}$ of data for large missing transverse energy caused by escaping neutralinos and several jets with high transverse energies. The derived $95 \%$ C.L. excluded region is shown on the $m_{\tilde{q}}-m_{\tilde{g}}$ plane in Fig. 2.10. In this figure, previous results from UA1 [52] and UA2 [53] together with the exclusion limits obtained at LEP, are shown. The search excludes gluino mass below $195 \mathrm{GeV} / \mathrm{c}^{2}$, independent of the squark mass, and for the case $m_{\tilde{g}} \approx m_{\tilde{q}}$, masses below $300 \mathrm{GeV} / \mathrm{c}^{2}$.

\footnotetext{
${ }^{11}$ In the next chapter, the different contributions for the particular points generated will be shown
} 
(a)

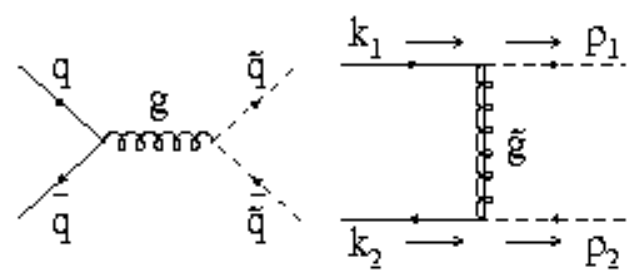

(b)
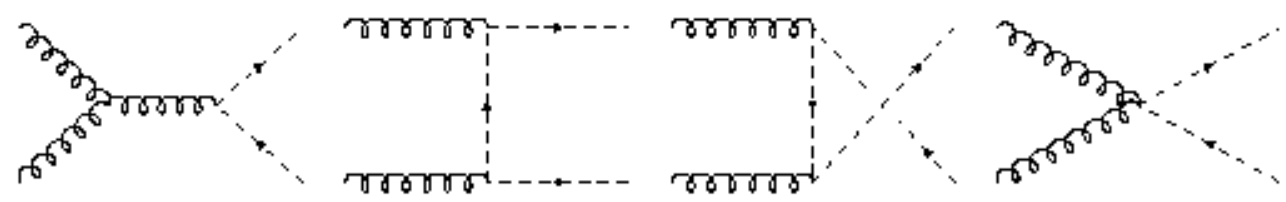

(c)
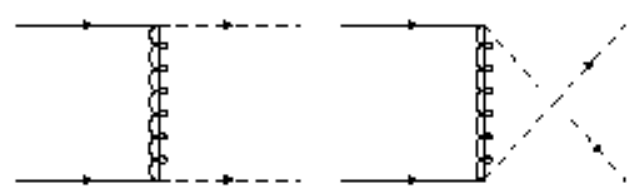

(d)
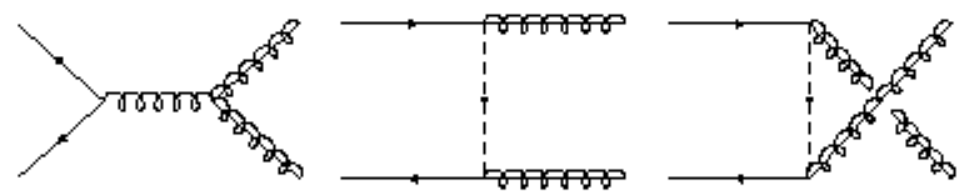

(e)
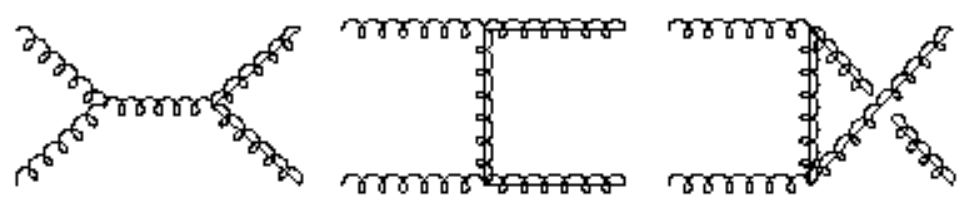

(f)
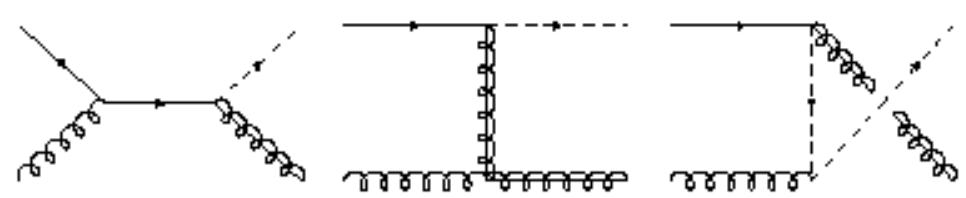

Fig. 2.8: Feynman diagrams for the production of squarks and gluinos in lowest order. The diagrams in (c) and the last diagram in (d) are result of the Majorana nature of gluinos. Some of the above diagrams contribute only for specific flavours and chiralities of the squarks. 

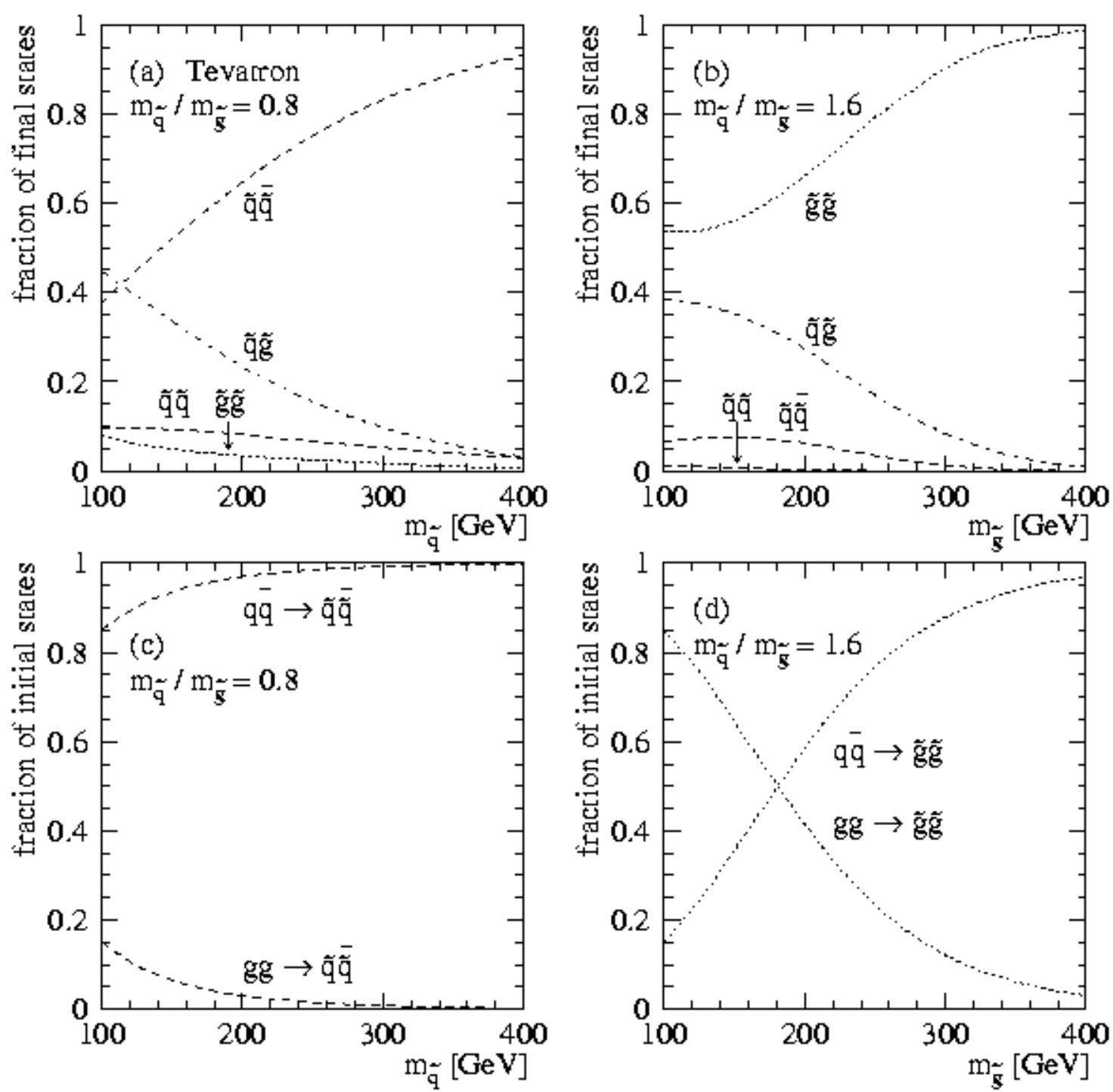

Fig. 2.9: The relative yields of squarks and gluinos in the final states at the Tevatron. The mass ratio $m_{\tilde{q}} / m_{\tilde{g}}$ is chosen to be (a) 0.8 and (b) 1.6. Also shown are the leading parton contributions for (c) $\tilde{q} \overline{\tilde{q}}$ and (d) $\tilde{g} \tilde{g}$ final states. 


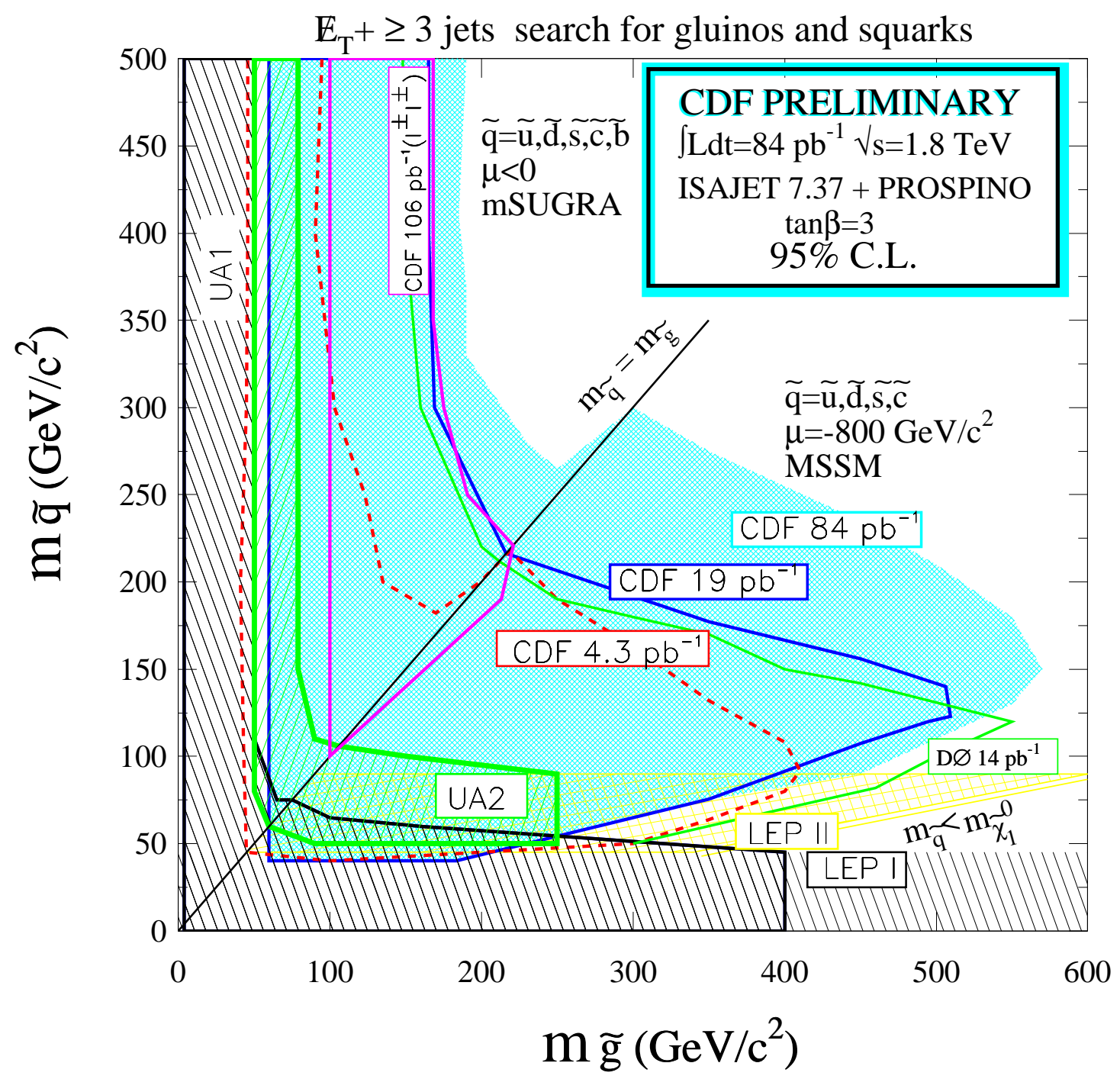

Fig. 2.10: $m_{\tilde{q}}-m_{\tilde{g}}$ exclusion plot at the $95 \%$ C.L assuming R-parity conservation for $\tan \beta=3$. Results form the previous searches are also shown. 



\section{Chapter 3}

\section{Experimental Setup}

Until the completion of the Large Hadron Collider (LHC) at CERN, the Tevatron provides the world's highest energy collisions. In this chapter, the Tevatron accelerator [54] and the Collider Detector at Fermilab (CDF) [55] in Run II, which provided the data used in this analysis, are described.

\subsection{The Tevatron in Run II}

The Tevatron is the proton antiproton superconducting collider at the Fermi National Accelerator Laboratory (FNAL) in Batavia, Illinois. It currently collides 36 proton on 36 antiproton bunches at a center-of-mass energy of $\sqrt{s}=1.96 \mathrm{TeV}$ with a bunch spacing of $396 \mathrm{~ns}$. With a circumference of about $6 \mathrm{~km}$, it is the world's first superconducting synchrotron and it hosts the experiments $\mathrm{CDF}$ and DØ in the two collision points. The Tevatron became operational in 1983 and during the Run I period (August 1991-February 1996) it delivered around $180 \mathrm{pb}^{-1}$ of data collected at $\sqrt{s}=1.8 \mathrm{TeV}$. The most important result from this period was the discovery of the top quark in 1995 [56]. Starting in 1996, both the accelerator and the experiments underwent significant upgrades in view of the Run II data-taking period, which began in 2001.

The acceleration of beams to $0.98 \mathrm{TeV}$ occurs in different stages at the Fermilab accelerator complex, as shown in Fig. 3.1. The different processes for protons and anti-protons are described below.

\section{Protons}

The acceleration cycle starts with negative hydrogen ions, $H^{-}$, which are accelerated to $750 \mathrm{keV}$ by a Cockroft-Walton electrostatic accelerator. The $H^{-}$ions enter a $150 \mathrm{~m}$ long Linear Acceler- 


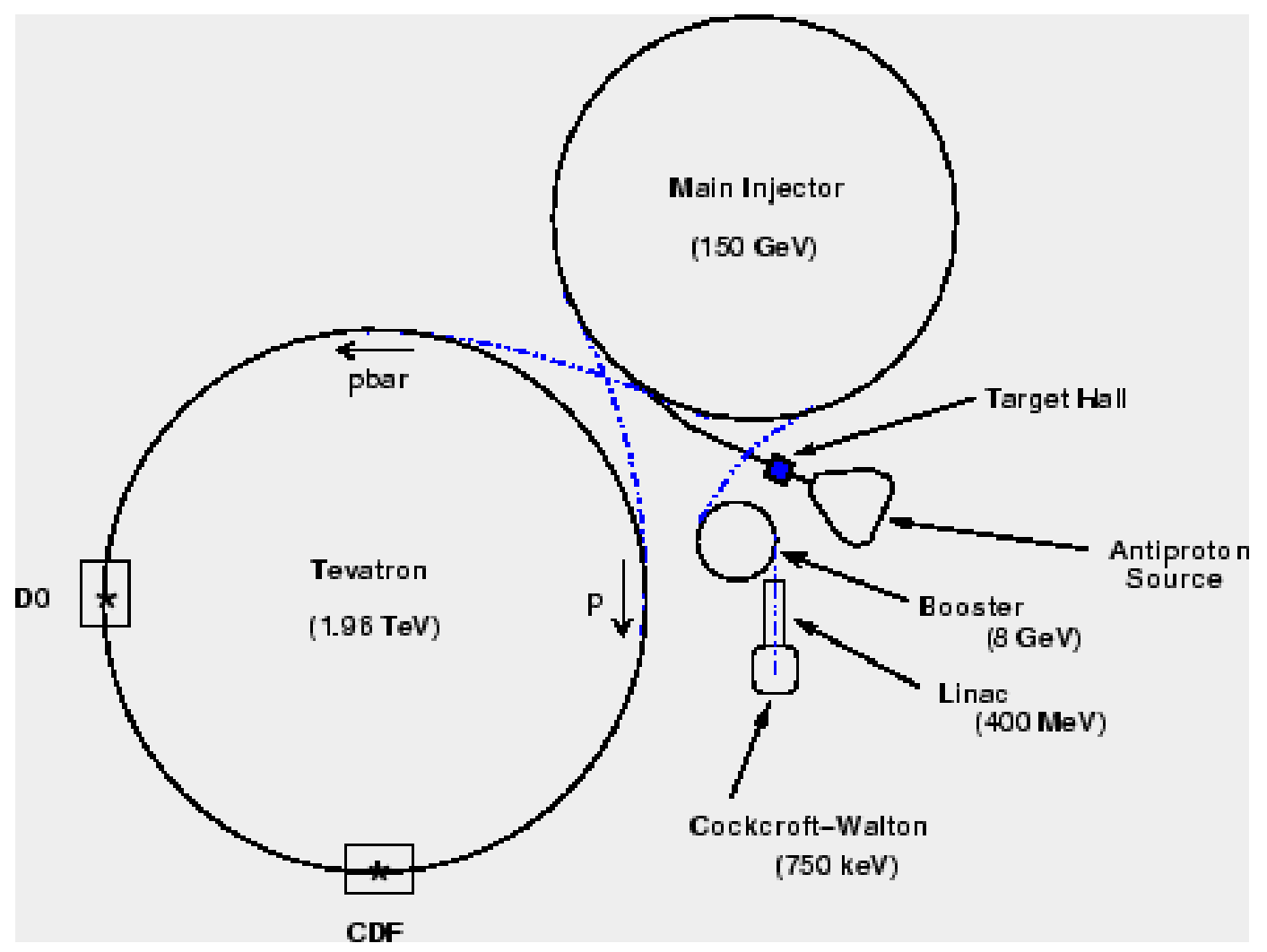

Fig. 3.1: Layout of the Fermilab accelerator complex.

ator (Linac) where they are accelerated by radio frequency (RF) accelerator cavities, running at $800 \mathrm{MHz}$ to an energy of $400 \mathrm{MeV}$. The accelerated $H^{-}$pass through a carbon foil which strips their electrons off and enter into the Booster. The Booster is the first synchrotron of the accelerator chain at Fermilab and it has a $75 \mathrm{~m}$ radius. The bare protons are merged into 84 bunches and accelerated to an energy of $8 \mathrm{GeV}$ prior to entering the Main Injector, which is another synchrotron with a circumference of $3 \mathrm{~km}$. In the Main Injector, the proton bunches are accelerated further to an energy of $150 \mathrm{GeV}$ and several bunches are merged into a single one (coalescing) to achieve high density prior to injection into the Tevatron. In the Tevatron there are 36 proton bunches which are accelerated to an energy of $980 \mathrm{GeV}$.

\section{Antiprotons}

From a technical point of view, a $p \bar{p}$ collider has the advantage that beams can circulate in opposite directions sharing the same magnet and vacuum system but the disadvantage that the production of an antiproton beam is significantly more complicated. The cycle starts with the extraction of a $120 \mathrm{GeV}$ proton beam from the main Injector onto a stainless steel target. This process produces antiprotons, among a variety of different particles, with an efficiency of $2 \cdot 10^{-5} /$ proton. 
The antiprotons are separated from the other by-products of the proton-nickel scattering using a bending magnet and they are collected and focused through a lithium lens. The system has a wide acceptance around $\bar{p}$ energies of $8 \mathrm{GeV}$. The resulting antiprotons are bunched preserving the structure of the initial protons. Since they have a large momentum spread, in both longitudinal and transverse directions, they need to go through an stochastic cooling process. This task is performed in two steps inside the Antiproton Source, which is a rounded triangular-shaped synchrotron facility consisting of a Debuncher and an Accumulator. The Debuncher is a synchrotron with a mean radius of $90 \mathrm{~m}$ which transforms the entering $\bar{p}$ into a continuous beam and cooled both transversely and longitudinally using RF manipulation. The resulting antiproton beam passes to the Accumulator, with a mean radius of $75 \mathrm{~m}$, where it is rebunched and accumulated. At this point, the antiprotons are still $8 \mathrm{GeV}$ and are sent to the Recycler. The Recycler is installed in the ceiling of the Main Injection ring and it is also used as an accumulator but with improved mechanisms to cool down the antiprotons and store them at a constant kinetic energy. Next step is to inject the antiprotons into the Main Injector for further acceleration to $150 \mathrm{GeV}$ from where they can enter to the Tevatron ring for the final acceleration to energies of $980 \mathrm{GeV}$.

\section{Final Stage}

Both protons and antiprotons circulate in opposite directions along the $6 \mathrm{~km}$ Tevatron ring. They circulate in three trains of twelve bunches. The trains are separated with $2.6 \mu$ s abort gaps and the bunches have a $396 \mathrm{~ns}$ separation. The low beta quads control the squeeze and the separators control the collisions at the CDF and DØ interaction points. The transverse profile of the interaction region can be approximately described by a circular Gaussian distribution with a typical RMS width of $30 \mu \mathrm{m}$. The longitudinal profile is also approximately gaussian with a typical RMS of $30 \mathrm{~cm}$.

The Tevatron performance has been improving along the years thanks to the different upgrades, specially in the antiproton chain. The improvements to the Debuncher and Accumulator stochastic cooling systems and the increase in the number of protons per pulse from the Main Injector contributed to decrease the time for antiproton accumulation from nearly a day at the beginning to the current two or three hours. In addition, the implementation of the electron cooling system in the Recycler, by which the antiproton beam is put in contact with a cooler electron beam, improved further the luminosity since it pushed the Accumulator to work at higher efficiency. According to the design, Tevatron is expected to have delivered more than $8 \mathrm{fb}^{-1}$ by 2009. 


\subsection{The Collider Detector at Fermilab}

\subsubsection{General Characteristics}

The Collider Detector at Fermilab (CDF II) [57] shown in Fig. 3.2 is a multipurpose experiment with azimuthal and forward-backward symmetry, designed to study high energy $p \bar{p}$ collisions. It combines precision charged particle tracking with fast projective calorimetry together with fine grained muon detection. The tracking system is contained inside a superconducting solenoid of $4.8 \mathrm{~m}$ length and $1.5 \mathrm{~m}$ radius which generates a $1.4 \mathrm{~T}$ magnetic field parallel to the beam axis. The muon and calorimetry systems are located outside the solenoid. The solenoid is made of an Al-stabilized NbTi superconductor and operated at liquid helium temperature which is able to carry currents of up to $5 \mathrm{kA}$. The magnetic field is uniform with an accuracy of $0.1 \%$ throughout the entire tracking volume.

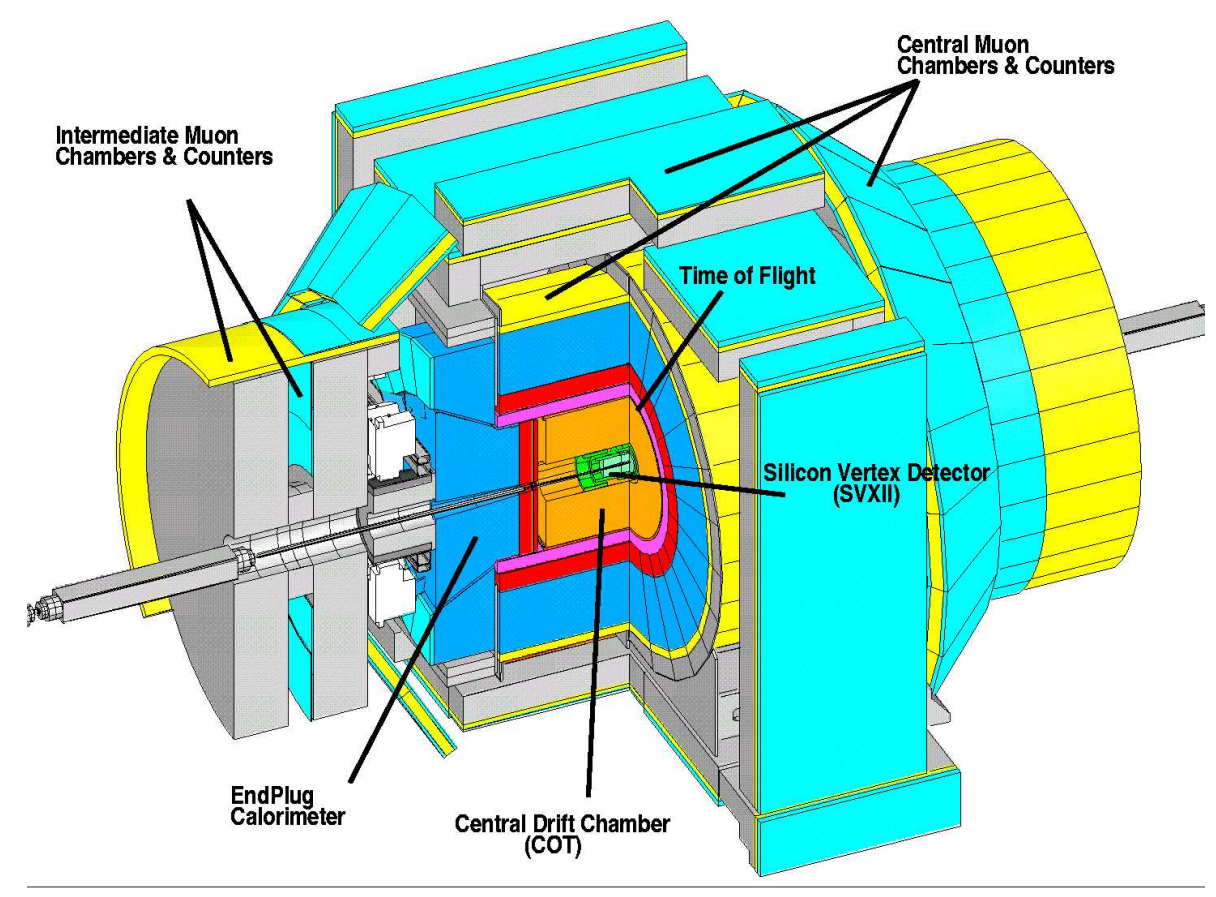

Fig. 3.2: Isometric view of the CDF Run II detector.

In the following sections, the different detector subsystems are briefly described in the sequence that an hypothetical particle coming from the interaction point would follow. At the end, the data acquisition system and the device to measure the luminosity are also presented. 


\subsubsection{Standard Definitions at CDF}

CDF II detector uses a cylindrical coordinate system $(r, \phi, z)$ with origin at the center of the detector. As shown in the diagram Fig. 3.3, the $z$-axis lays along the nominal direction of the proton beam and the $y$-axis points upwards. Since in hadron colliders, the distribution of the energy and longitudinal momentum of the partons inside the hadrons are unknown, only transverse quantities, such as the transverse energy $\left(E_{T}=E \sin \theta\right)$ or the transverse momentum $\left(p_{T}=p \sin \theta\right)$ are useful.

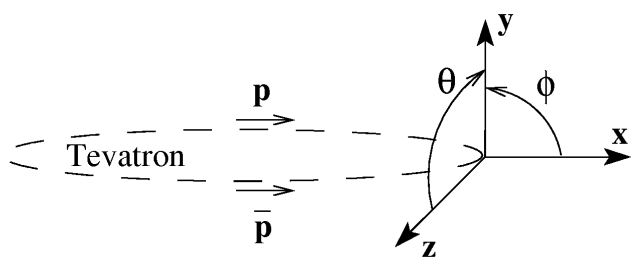

Fig. 3.3: The CDF coordinate system.

The rapidity, $y$, of a particle is given by $y \equiv \frac{1}{2} \ln \left[\frac{E+p_{z}}{E-p_{z}}\right]$ and it is invariant under a Lorentz boost transformation. The pseudo-rapidity, $\eta$, defined as $\eta \equiv-\ln \tan \left[\frac{\theta}{2}\right]$ equals the rapidity $y$ in the massless approximation (limit $E \gg m c^{2}$ ) and it is extensively used because it has a direct geometric interpretation. Fig. 3.4 shows the different $\eta$ coverage for some of the individual components of the CDF detector. If $\eta$ is measured from the detector center instead of from the interaction point, then this quantity is denoted as $\eta_{d}$.

\subsubsection{The Silicon detector}

The silicon detector [58] constitutes the innermost part of the CDF tracking systems (shown in Fig. 3.4) and provides an extension for tracking down to 2.8 in pseudorapidity. It consists of three subdetectors, each using different silicon sensor designs and layouts. All the CDF II silicon tracking detectors are implemented as microstrip detectors. The typical distance between two strips is about $60 \mu \mathrm{m}$ and the charge deposition from a single particle is read out by one or more strips. There are two types of microstrip detectors: single and double-sided. The latter, have both sides of the p-n junction segmented into strips and present the benefit that one (p) side has strips parallel to the $z$ direction, providing $r-\phi$ position measurements, while the other (n) side has strips at an angle (stereo angle) with respect to the $z$ direction and provides $z$ position information.

The silicon system is divided in three main subsystems: Layer 00 (L00), Silicon Vertex Detector (SVX) and Intermediate Silicon Layers (ISL). The L00 serves to improve the track impact parameter resolution and it is distributed around the beam vacuum pipe at a minimum radius of $1.35 \mathrm{~cm}$. The SVX is used to obtain precise position measurements of the path of a charged 


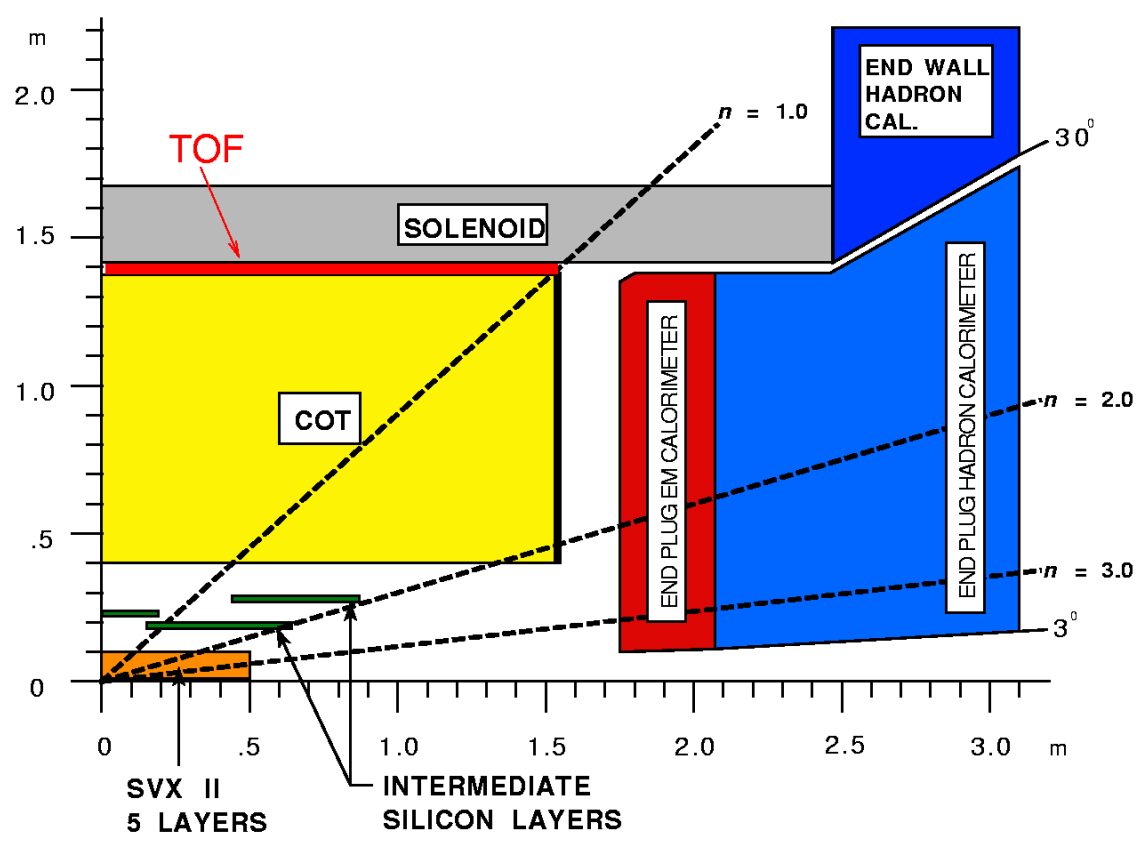

Fig. 3.4: Longitudinal view of the CDF Run II tracking system.

particle $\left(z_{0}\right.$ resolution of $\left.70 \mu \mathrm{m}\right)$. It is constituted by three long cylindrical barrels, subdivided in wedges and layers, which can combine $r-\phi$ measurements with information from stereo angles for three-dimensional track reconstruction. Finally, the ISL consists of three separate silicon layers and serves as a link between the inner silicon tracking region and the outer wire tracker.

\subsubsection{The Central Outer Tracker}

The Central Outer Tracker (COT) [59] is a multiwire, open cell cylindrical drift chamber using a read out that can record multiple hits from each sense wire. Due to the hadron collider characteristics, the COT is designed to provide more accurate measurements from the $r-\phi$ plane (transverse momentum) than from the $r-z$ plane. The COT is located just above the ISL, with an inner radius of $43.3 \mathrm{~cm}$ and an outer radius of $132.3 \mathrm{~cm}$. It is $310 \mathrm{~cm}$ long, covering a pseudorapidity range $\left|\eta_{d}\right|<1$. The COT is radially divided into 8 "superlayers" (SL). Each SL is azimuthally divided into a number of "supercells". These supercells have a maximum drift distance that is approximately the same for all superlayers. Therefore, the number of supercells in a given SL scales approximately with the radius.

As shown in Fig. 3.5, each of the supercells is limited by two $10 \mu \mathrm{m}$ gold-coated mylar grounded field sheets and contains a set of $40 \mu \mathrm{m}$ gold-plated tungsten wires, alternating 13 potential wires with twelve sense wires. The entire COT contains 30,240 sense wires, each of 
them connected to the readout electronics to collect the charge left by the ionisation of the incident charged particle. The field wires have lower voltages and shape the electric field to achieve maximum uniformity within the cell, allowing for a constant drift field. The nominal spacing between sense and potential wires is determined by simulation to be around $0.36 \mathrm{~cm}$. The eight superlayers of the COT alternate between stereo and axial. In an axial layer, the wires and field sheets are parallel to the $z$ axis, providing only $r-\phi$ information. In stereo layers, the wires and field sheets are arranged with a stereo angle of $\pm 2^{\circ}$ and provide additional $z$ information.

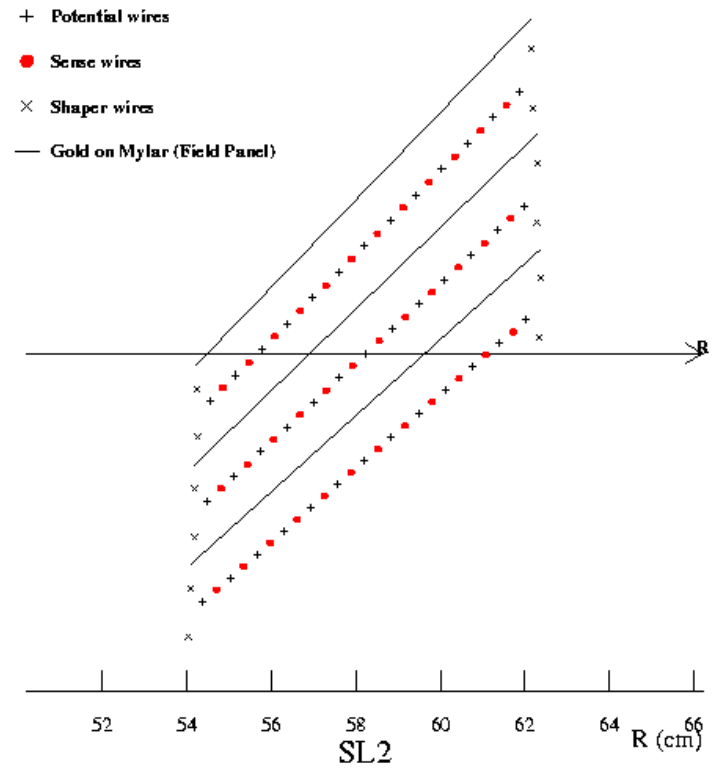

Fig. 3.5: Wire layout in a COT supercell of SL2.

Due to the magnetic field in which the COT is immersed, electrons drift at a Lorentz angle of $\sim 35^{\circ}$. Thus, each supercell is tilted by this angle with respect to the radial direction to compensate for this effect and minimise the time window in which the drifting electrons arrive to the sense wires. In addition, in the middle of each wire along the $z$ direction, there is a mechanical spacer made of polyester/fiber glass to limit the stepping of wires out of the plane due to electrostatic forces.

As shown in Fig. 3.6, particles originating from the interaction point which have $|\eta|<1.3$ pass through four or more superlayers. Particles with $|\eta|<1$ pass through all 8 superlayers. The COT has a maximum acceptance of $|\eta|<2$.

The proportional drift chamber is filled with Ar:C2H6 (50:50) mixture with a small admixture of isopropyl alcohol and oxygen to prevent aging. This mixture is chosen to achieve a uniform gain $\left(\approx 2 \cdot 10^{4}\right)$ and drift field with relatively high velocities $(\approx 100 \mu \mathrm{m} / \mathrm{ns})$ for all the sense wire drift cells. The maximum drift distance in a cell is $0.88 \mathrm{~cm}$ which allows for a maximum drift 


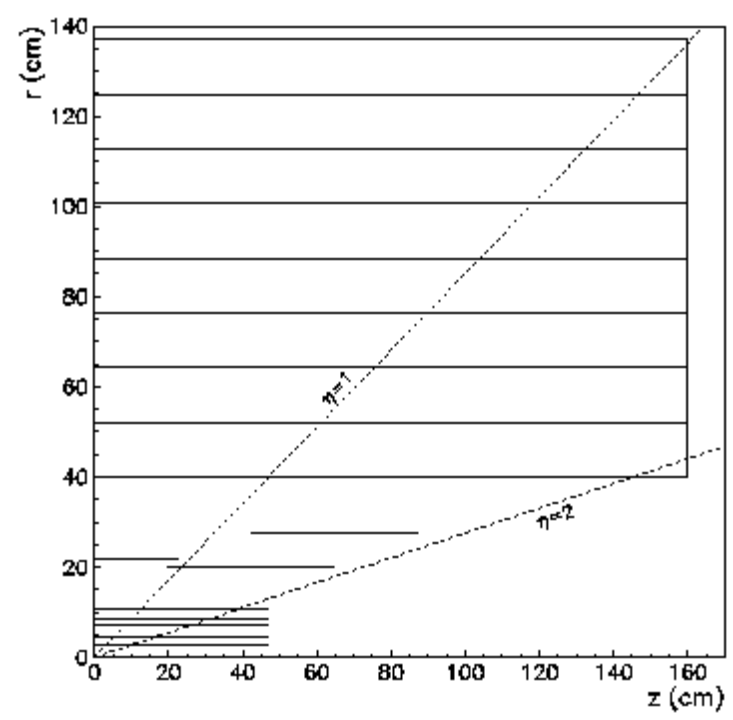

Fig. 3.6: Schematic $r-z$ view of the $\eta$ coverage of the inner and outer tracker (SVX II, ISL and COT).

time of $100 \mathrm{~ns}$, well enough inside the bunch crossing time window of $396 \mathrm{~ns}$. The COT single-hit resolution has been measured in situ using $Z$ boson decays into muon pairs, $Z \rightarrow \mu \mu$, to be about $140 \mu \mathrm{m}$. The momentum resolution has been measured to be $\sigma_{p_{T}} / p_{T}^{2} \approx 1.5 \times 10^{-3}[\mathrm{GeV} / \mathrm{c}]^{-1}$ using muon cosmic rays.

\subsubsection{The Time of Flight}

The Time Of Flight (TOF) detector [60] is used to distinguish low momentum pions, kaons and protons by measuring the time they take to travel from the primary vertex to the system. The TOF lays outside the tracking system, still inside the superconducting magnetic coil. This system consists of 216 scintillating bars arranged into a barrel around the COT cylinder. Each bar has a photomultiplier tube attached at both ends in order to detect the light coming from the energy deposition of the particles. The readout electronics perform both time and amplitude digitisation of the signal. The timing resolution of the TOF system is currently about $110 \mathrm{ps}$ for particles crossing the bar exactly in front of one of the phototmultiplier tubes. Since light attenuates while travelling through the scintillator material, particles passing near the photomultiplier tube have better timing resolution than those which are farther away.

\subsubsection{The Calorimeters}

The primary purpose of the CDF calorimeters is to measure the energy of charged and neutral particles. A schematic view is shown in Fig. 3.7. The CDF calorimeters instrument two regions: 
central $\left(\left|\eta_{\text {det }}\right|<1\right)$ and forward $\left(1.1<\left|\eta_{\text {det }}\right|<3.6\right)$.

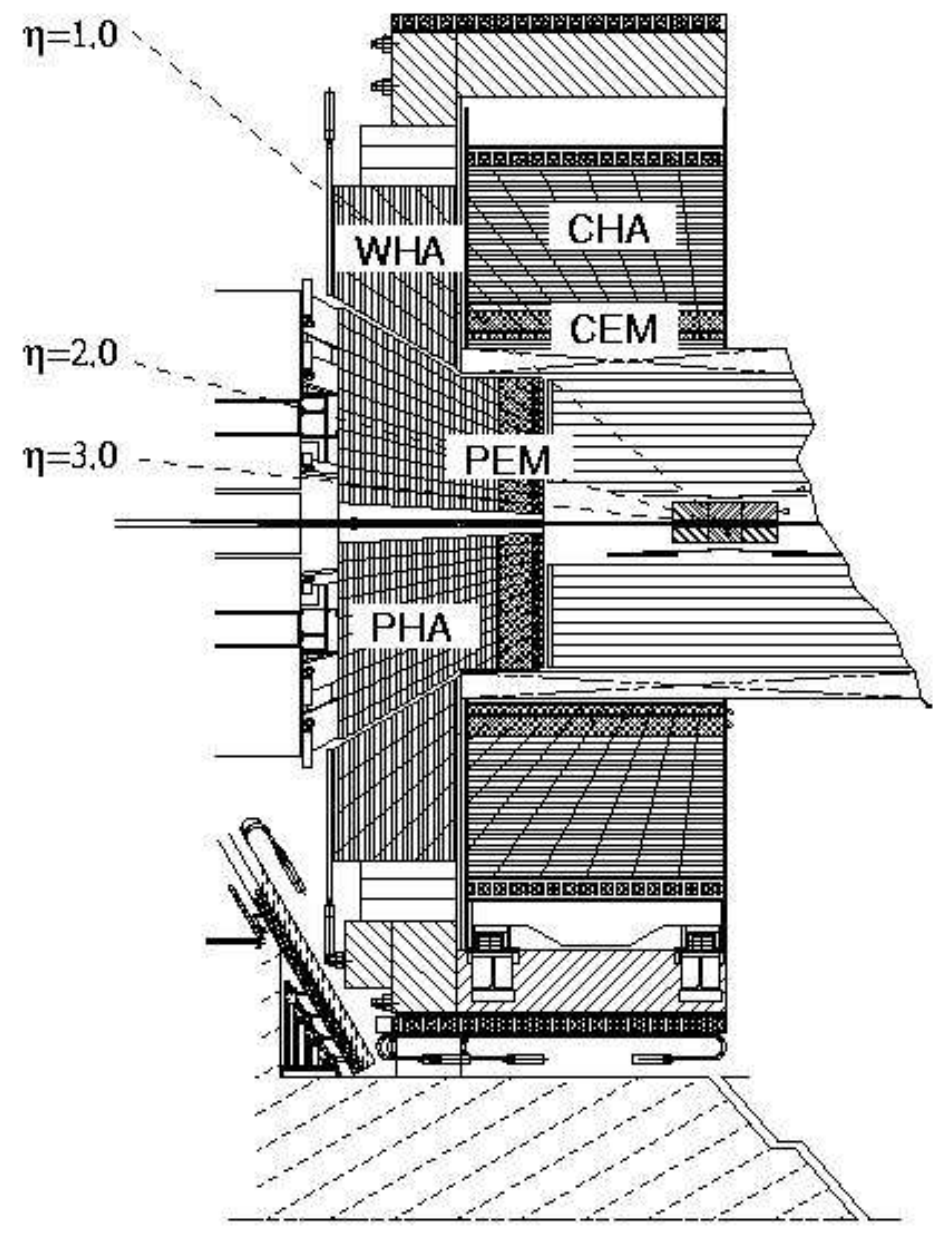

Fig. 3.7: Elevation view of one half of the CDF detector displaying the different components of the CDF calorimeter.

\section{Central Calorimeter}

The central calorimeter is subdivided into an inner detector, called the central electromagnetic calorimeter (CEM) [61], which is designed to absorb the electromagnetic particles as well as pions, and two outer detectors, called the central hadronic and endwall calorimeters (CHA, WHA) [62], which are designed to stop the strong interacting particles.

These scintillator-based sampling calorimeters are set outside the solenoid and they are arranged in the form of projective towers pointing to the center of the detector. Each tower is a set of plastic scintillator tiles interleaved with lead (steel) sampling material in the case of CEM (CHA-WHA). Every tower covers approximately 0.1 unit in pseudorapidity and $15^{\circ}$ in azimuthal 
angle. Thus, the central calorimeters are divided in 24 azimuthal slices. As shown in Fig. 3.7, in each of these slices there exist 12 towers completely in the CHA calorimeter, 6 towers at the WHA calorimeters and 6 towers are shared between both subsystems. Towers in CEM match those of the hadron calorimeters.

The CEM thickness is 18 radiation lengths ${ }^{1}\left(X_{0}\right)$ and the CHA-WHA thickness is 4.7 interaction lenghts ${ }^{2}\left(\lambda_{I}\right)$. The light produced in response to the energy deposited is collected using wave-length-shifting fibers and derived to the photomultipliers tubes (PMTs) to produce around 40 photoelectrons per GeV. The light guide mapping for CHA is sketched in Fig. 3.8.

The energy resolution for each section was measured in the testbeam and can be parameterised as

$$
\left(\frac{\sigma}{E}\right)^{2}=\left(\frac{\sigma_{1}}{\sqrt{E}}\right)^{2}+\left(\sigma_{2}\right)^{2}
$$

where the first term comes from sampling fluctuations and the photostatistics of PMTs and the second term comes from the non-uniform response of the calorimeter. In the CEM, the energy resolution for high energy electrons and photons at normal incidence is $\frac{\sigma}{E_{T}}=\frac{13.5 \%}{\sqrt{E_{T}}} \oplus 2 \%$, where the energy is expressed in GeV. In CHA and WHA detectors, charged pions were used to obtain the energy resolution and it was found to be $\frac{\sigma}{E_{T}}=\frac{50 \%}{\sqrt{E_{T}}} \oplus 3 \%$ and $\frac{\sigma}{E_{T}}=\frac{75 \%}{\sqrt{E_{T}}} \oplus 4 \%$, respectively. In addition, the overall calorimeter has an important role for muon identification. The average energy loss per Minimum Ionising Particle (MIP) in the calorimeter is around 0.5 (1.6) GeV for electromagnetic (hadronic) parts.

\section{Plug Calorimeter}

The forward or "plug" calorimeters [63] are also divided in electromagnetic (PEM) and hadronic (PHA) parts. The plug calorimeters are completely new from Run II upgrade, contributing to a more hermetic detector and replacing Run I gas calorimeters.

These calorimeters are also arranged in the form of projective towers pointing to the center of the detector. Each tower is a set of plastic scintillator tiles interleaved with lead (iron) sampling material in the case of PEM (PHA). The $\eta$ coverage of the towers vary depending on the pseudorapidity region ${ }^{3}$ from 0.1 to 0.6 and the $\phi$ coverage vary from $7.5^{\circ}$ in the region $1.1<|\eta|<2.1$ to $15^{\circ}$ in the region $2.1<|\eta|<3.6$. There exist 48 azimuthal modules. Towers in the PEM match those of the PHA (except for the lowest $\eta$ PEM tower which does not have a corresponding PHA tower).

\footnotetext{
${ }^{1}$ The radiation length $X_{0}$ describes the characteristic amount of matter traversed for high energy electrons in order to lose all but $1 / \mathrm{e}$ of its energy by bremsstrahlung.

${ }^{2} \mathrm{An}$ interaction length is the average distance a particle will travel before interacting with a nucleus.

${ }^{3}$ The segmentations optimise $e^{ \pm}$identification in $b / \bar{b}$ jets ( $b \rightarrow e+X$ processes) [57].
} 


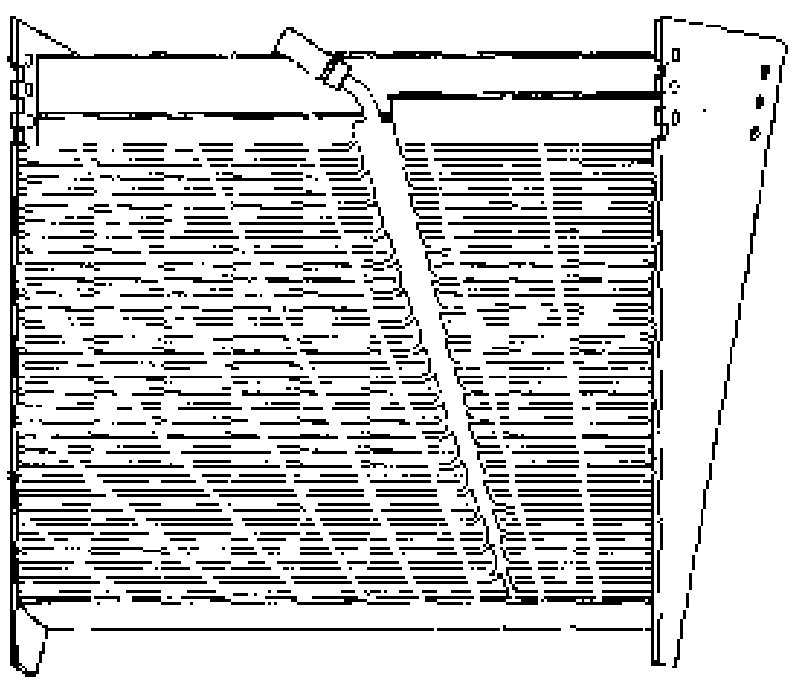

Fig. 3.8: Central hadronic calorimeter module. The light scheme is sketched.

The PEM thickness is $23 X_{0}$ and the PHA thickness is $6.8 \lambda_{I}$. The light produced in response to the energy deposited is collected using wave-length-shifting fibers and derived to the photomultipliers tubes (PMTs) to produce around 300 photoelectrons per $\mathrm{GeV}$. The energy resolution for the plugs was determined in the test beam to be $\frac{\sigma}{E}=\frac{16 \%}{\sqrt{E}} \oplus 1 \%$ for PEM and $\frac{\sigma}{E}=\frac{80 \%}{\sqrt{E}} \oplus 5 \%$ for PHA where the resolution is given as a function of the total energy.

Table 3.1 shows in detail some of the most important characteristics of the CDF calorimetry. As a final remark, mention that there are two main uninstrumented regions. One is in the CEM where there is one azimuthal wedge module that it is notched to allow a "chimney" for access to the CDF superconducting solenoid. The other is the cracks at $\eta=0$ and $|\eta|=1.1$ which allow the junction between the two symmetrical central modules and between the WHA and the PHA, respectively.

\begin{tabular}{|c|c|c|c|c|c|}
\hline \hline Calorimeter & CEM & CHA & WHA & PEM & PHA \\
\hline \hline Absorber & Lead & Steel & Steel & Lead & Iron \\
\hline $\begin{array}{c}\text { Segmentation } \\
(\eta \times \phi)\end{array}$ & $0.1 \times 15^{\circ}$ & $0.1 \times 15^{\circ}$ & $0.1 \times 15^{\circ}$ & $\begin{array}{c}(0.1-0.6) \times \\
\left(7.5^{\circ}-15^{\circ}\right)\end{array}$ & $\begin{array}{c}(0.1-0.6) \times \\
\left(7.5^{\circ}-15^{\circ}\right)\end{array}$ \\
\hline Num. Towers $(\eta \times \phi)$ & $20 \times 24$ & $9 \times 24$ & $6 \times 24$ & $12 \times 24(48)$ & $11 \times 24(48)$ \\
\hline Thickness & $18 X_{0}, 1 \lambda_{I}$ & $4.7 \lambda_{I}$ & $4.7 \lambda_{I}$ & $23 X_{0}, 1 \lambda_{I}$ & $6.8 \lambda_{I}$ \\
\hline Resolution $(\%)$ & $14 / \sqrt{\mathrm{E}_{\mathrm{T}}} \oplus 2$ & $50 / \sqrt{\mathrm{E}_{\mathrm{T}}} \oplus 3$ & $75 / \sqrt{\mathrm{E}_{\mathrm{T}}} \oplus 4$ & $16 / \sqrt{E} \oplus 1$ & $80 / \sqrt{E} \oplus 5$ \\
\hline \hline
\end{tabular}

Tab. 3.1: CDF II calorimetry summary. 


\section{The Showermax and Pre-Radiator detectors}

The central and forward parts of the calorimeter have their own shower profile detector positioned at the expected maximum of the lateral shower profile (approximately at $6 X_{0}$ ). These Central Electromagnetic Showermax (CES) [64] or Plug Electromagnetic Showermax (PES) [65] are designed to measure the position of electron and photon showers and to help on separating single electrons and photons from the photons produced in $\pi^{0} \rightarrow \gamma \gamma$ decays.

The Central Pre-Radiator (CPR) [66] is located at the inner face of the central calorimeter and consists of several multiwire proportional chambers which sample the electromagnetic shower that begin in the solenoid magnetic material (approximately $1 X_{0}$ ) in front of them. The Plug PreRadiator (PPR) [67] serves a similar purpose but it is located in front of the plug calorimeters.

\subsubsection{The Muon System}

The CDF II muon system [68] consists of four subsystems, which are all functionally similar, that cover the region of $|\eta|<2$ and $2 \pi$ in azimuthal: the central muon chambers (CMU), the central muon upgrade chambers (CMP), the central muon extension (CMX) and the intermediate muon system (IMU). These units are located outside the calorimeter systems, as shown in Fig. 3.2, and use the calorimeter steel and the magnet return yoke as absorbers for showering particles. The systems consists on drift cells and scintillation counters which are used to reconstruct the tracks from minimum ionising particles. These tracks are matched using dedicated algorithms with the COT information in order to reconstruct the full trajectory of the muons.

\subsubsection{The Trigger System}

The collision rate at the Tevatron is much higher than the rate at which data can be stored on tape. The role of the trigger is to efficiently extract the most interesting physics events from the large number of $p \bar{p}$ collisions. The CDF trigger system has a three level architecture as shown in Fig. 3.9. Each level provides a rate reduction sufficient to allow for processing in the next level with minimal deadtime.

Level 1 (L1) uses designed hardware to make decisions based on simple physics quantities within events using a subset of the detector information. As shown in Fig. 3.10, three different streams of information allow L1 to make a decision: calorimeter objects that may be further reconstructed into electrons, photons or jets; track segments in the muon detector and tracking data to identify tracks which can be linked to objects in the calorimeter or muon detector. The L1 trigger decision takes place $5.5 \mu \mathrm{s}$ after a collision and it works in parallel through a pipeline that can store up to 14 bunch crossings. This buffered data is needed in order to accommodate 


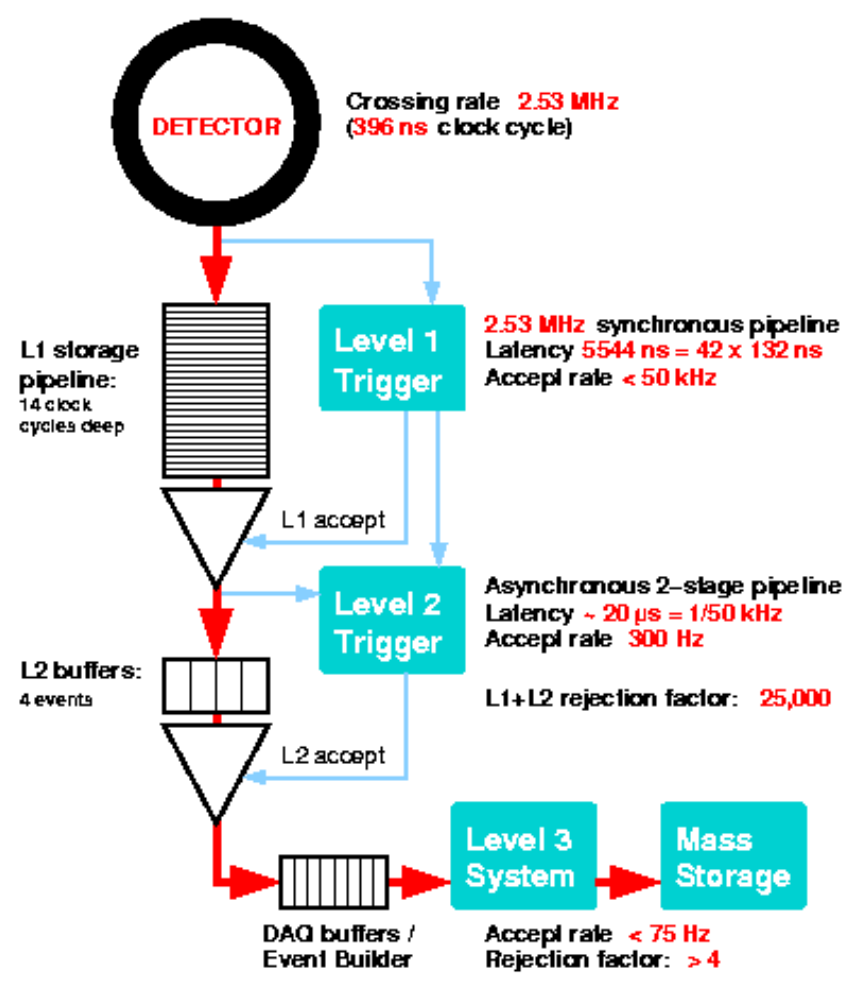

Fig. 3.9: The CDF Run II Trigger and Data Acquisition System.

the average input rate of $1.7 \mathrm{MHz}$, determined by the Tevatron bunch configuration. After L1, the event rate is reduced to less than $50 \mathrm{kHz}$.

The level 2 (L2) is a combination of hardware and software trigger that perform limited event reconstruction using programmable processors. These events are stored in one of four asynchronous buffers and the decision whether they are accepted or not is based on cluster algorithms, shower information from Showermax detectors and combined tracking information from L1 and from SVX II, which is crucial in order to trigger on different tracking features like the impact parameter. This level of decision takes approximately $25 \mu$ s and further reduces the event rate to approximately $300 \mathrm{~Hz}$.

The level 3 (L3) consists of two components: an "event builder" and a Linux PC farm. As shown in Fig. 3.11 the detector readout from the L2 buffers is received via an Asynchronous Transfer Mode (ATM) switch and distributed to 16 PC nodes. The main task of these nodes is to assemble all the pieces of the same event as they are delivered from different subdetector systems through the ATM switch. The event is then passed to a processor node consisting on a separate dual-processor PC. There are about 150 processor nodes and each of the two CPUs processes a single event at a time. The L3 decision is based on a near-final quality reconstruction which, if it 


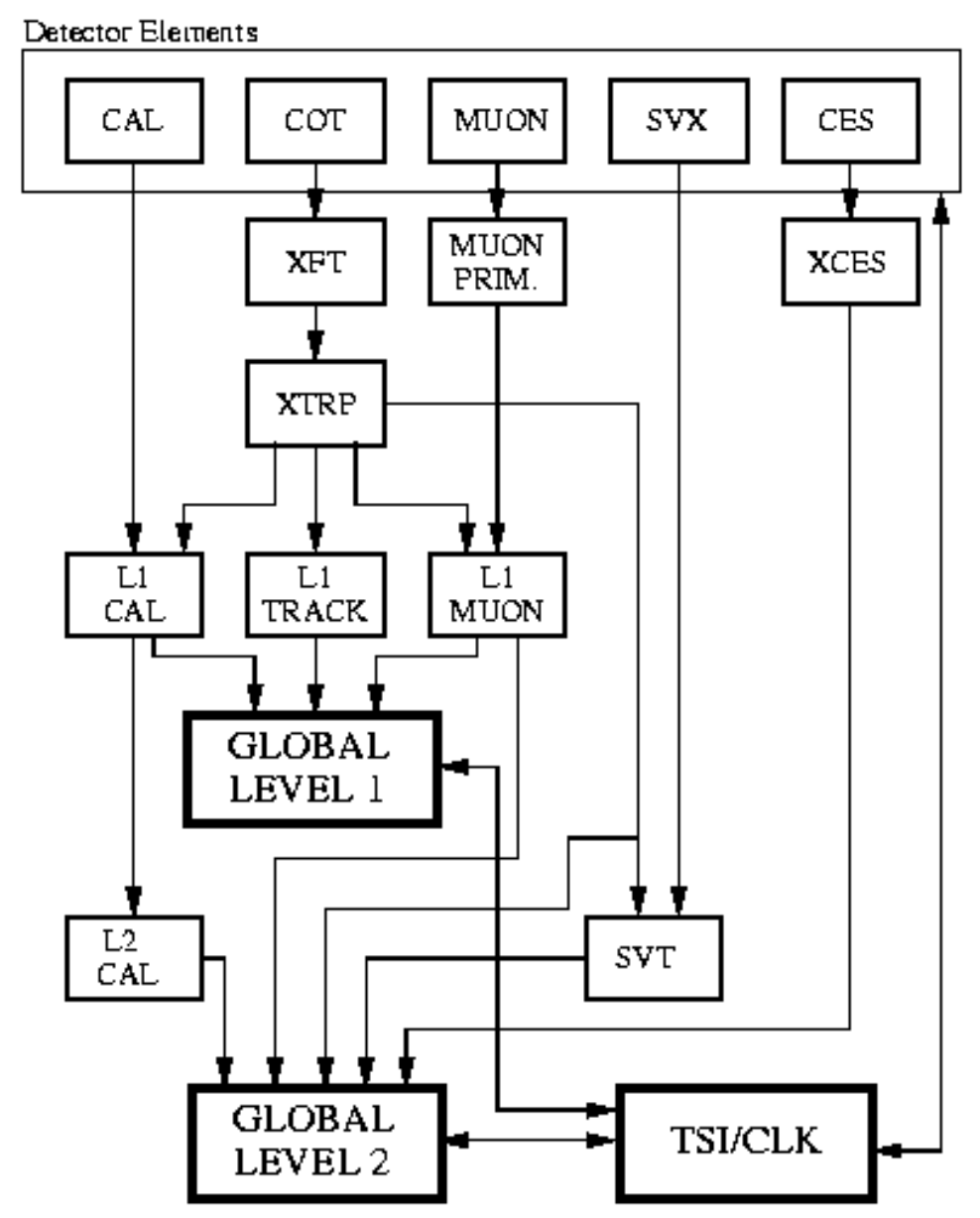

Fig. 3.10: Block diagram of the CDF Run II Trigger System.

passes certain criteria, it is sent to the Consumer Server / Data Logger (CS/DL) system for storage first on disk and then on tape. This level of decision reduces the event rate to approximately $75 \mathrm{~Hz}$.

\subsubsection{CLC and luminosity measurement}

The luminosity $(L)$ at CDF is determined from the rate of inelastic $p \bar{p}$ interactions in the Cherenkov Luminosity Counters (CLC) [69] detector. The CLC occupy the conical holes $(3.75<|\eta|<4.75)$ between the plug calorimeters and the beampipe as shown in Fig. 3.12. It is composed of 48 thin, long, gas-filled, Cherenkov counters. They are arranged around the beam pipe in three concentric layers, with 16 counters each, and pointing to the center of the interaction region. The counters are mounted inside a thin pressure vessel made of aluminium and filled with isobutane. The Cherenkov angle is $3.1^{\circ}$ and the momentum threshold for light emission is $9.3 \mathrm{MeV} / \mathrm{c}$ for elec- 


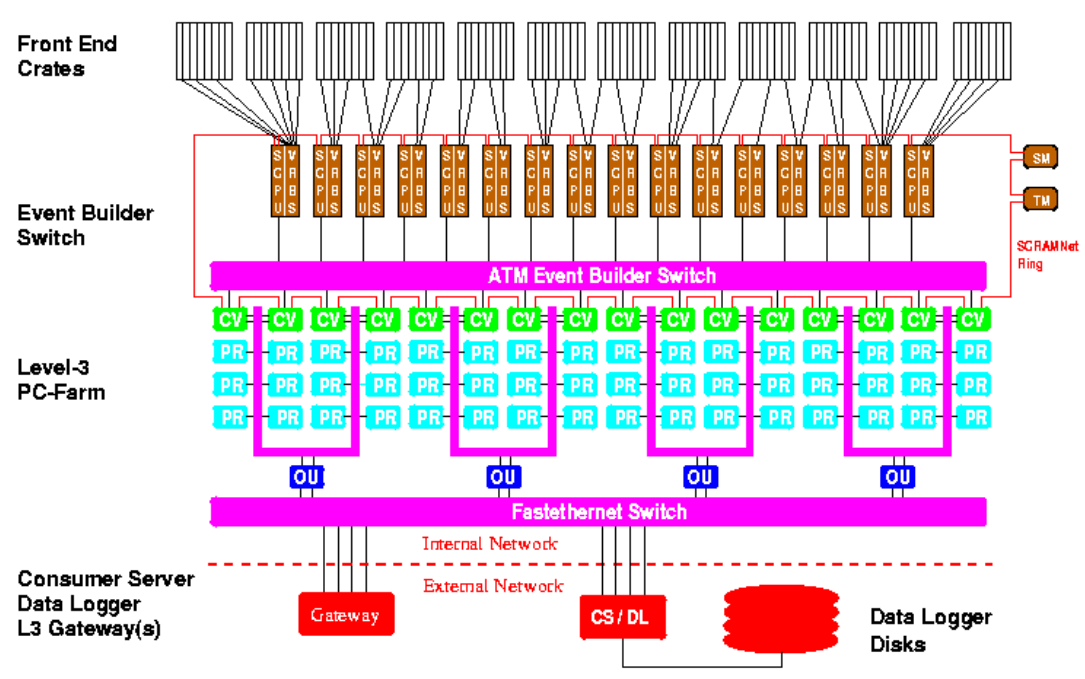

Fig. 3.11: Event Builder and L3 filtering. Data from the front end crates pass through ATM switches to the converter nodes. Here, the events are assembled and passed to the processor nodes. The accepted events are passed to output nodes which send them to the Consumer Server and Data Logging systems (CS/DL).

trons and $2.6 \mathrm{GeV} / \mathrm{c}$ for pions.

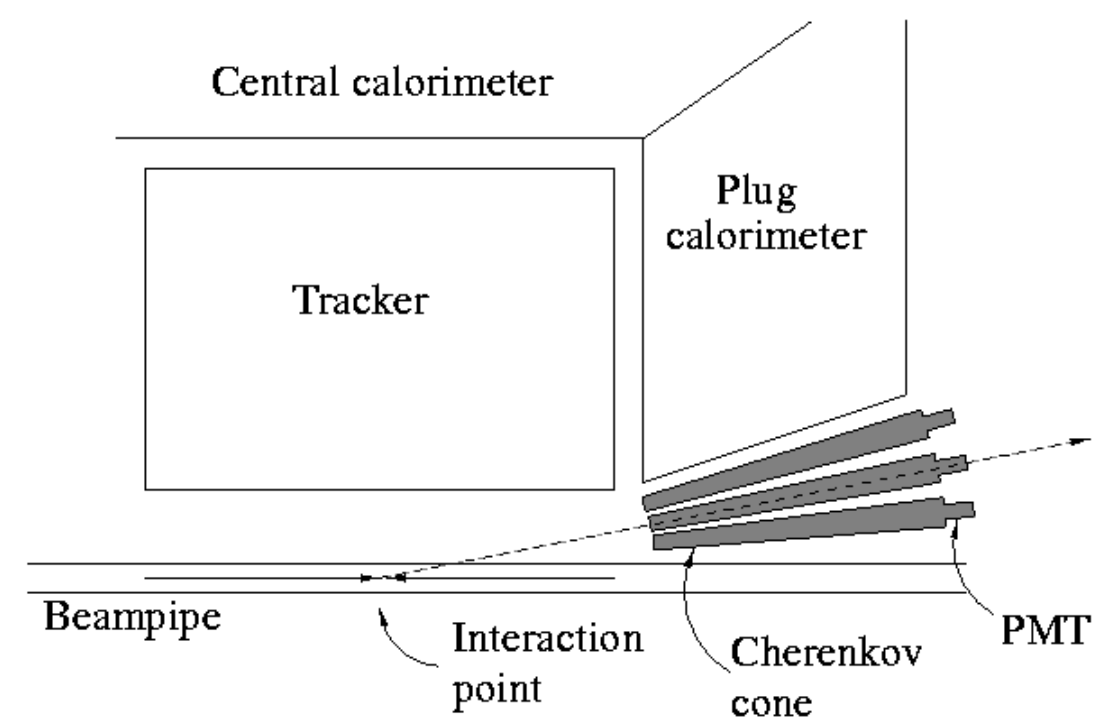

Fig. 3.12: Schematic view of the luminosity monitor inside a quadrant of CDF. It is located at $|\theta|<3^{\circ}$.

The CLC is designed to measure the average number of inelastic interactions per bunch crossing, $\mu$, within a few percent, up to the high luminosity regimes expected for the Tevatron. Then, the luminosity is extracted using: 


$$
\mu \cdot f_{B C}=\sigma_{i} \cdot L
$$

where $\sigma_{i}$ is the inelastic proton-antiproton scattering cross section ${ }^{4}$ and $f_{B C}$ is the frequency of bunch crossing, which is on average $1.7 \mathrm{MHz}$ for $36 \times 36$ bunch operations.

Since the number of interactions $n$ per bunch crossing follows Poisson statistics with mean $\mu$, one can have a good estimator for $\mu$ measuring the probability of empty bunch crossings $n=0$ :

$$
\mathcal{P}(0)=e^{-\mu} .
$$

An empty bunch crossing is observed when there are less than two tubes with signals above threshold in either module of the CLC. The measured fraction of empty bunch crossings is corrected for the CLC acceptance and the value of $\mu$ is calculated. The total systematic uncertainty on the luminosity [70] is about 6\%, which originates from uncertainties in the acceptance (4.4\%) and from the inelastic cross section normalisation (4\%).

\subsubsection{Data Quality Monitoring and Validation}

Part of the events from the collisions undertake some quality controls to ensure the different subdetector systems were in good conditions during data-taking. This series of controls involve statistical tests of different levels of complexity for some of the most sensitive variables. These tests are implemented online, to resolve possible problems in short time, and offline, when a careful reconstruction of the whole event is performed with the final calibrations.

Data is validated in "runs". Several lists ("GoodRunList"), specifying which of the runs pass certain quality criteria to be used for different kind of analysis, are made available. See Appendix A for more information about this system.

\footnotetext{
${ }^{4}$ The proton-antiproton inelastic cross section at the Tevatron is $\sigma_{i}=59.3 \mathrm{mb}$.
} 


\section{Chapter 4}

\section{Analysis Method}

\subsection{Data Pre-selection}

This work is based on $371 \mathrm{pb}^{-1}$ of Run II data collected before the beginning of 2005. The run number is in the 138809 - 186598 range. Some basic filters for data quality were enforced to ensure the data were collected with tracking and calorimeter systems working properly (see Appendix A). There are roughly ten million events in this sample.

\subsubsection{Trigger Path}

The three-level trigger logic that was employed to collect the event sample requires the presence of at least two jets in the final state together with large $\mathbb{E}_{T}$. This trigger is called "MET35". At L1 and L3 different $\mathbb{E}_{T}$ thresholds are required whereas the criteria to pass L2 is the presence of two calorimeter clusters of at least $10 \mathrm{GeV}$.

\begin{tabular}{|c|c|c|}
\hline \hline Trigger Level & requirement & Prescale \\
\hline L1 & MET 25 & 1 \\
\hline L2 & TWO-JET10 .and. L1-MET25 & 1 \\
\hline L3 & MET 35 & 1 \\
\hline \hline
\end{tabular}

Tab. 4.1: Summary of the MET35 trigger logic used in collecting the data.

The stability of the trigger cross section versus time was studied. Fig. 4.1 shows the effective cross section of the MET35 trigger versus run number. After some pre-selection cuts described in Section 4.1.2, no significant dependence of the measured cross section with the run number was observed. 


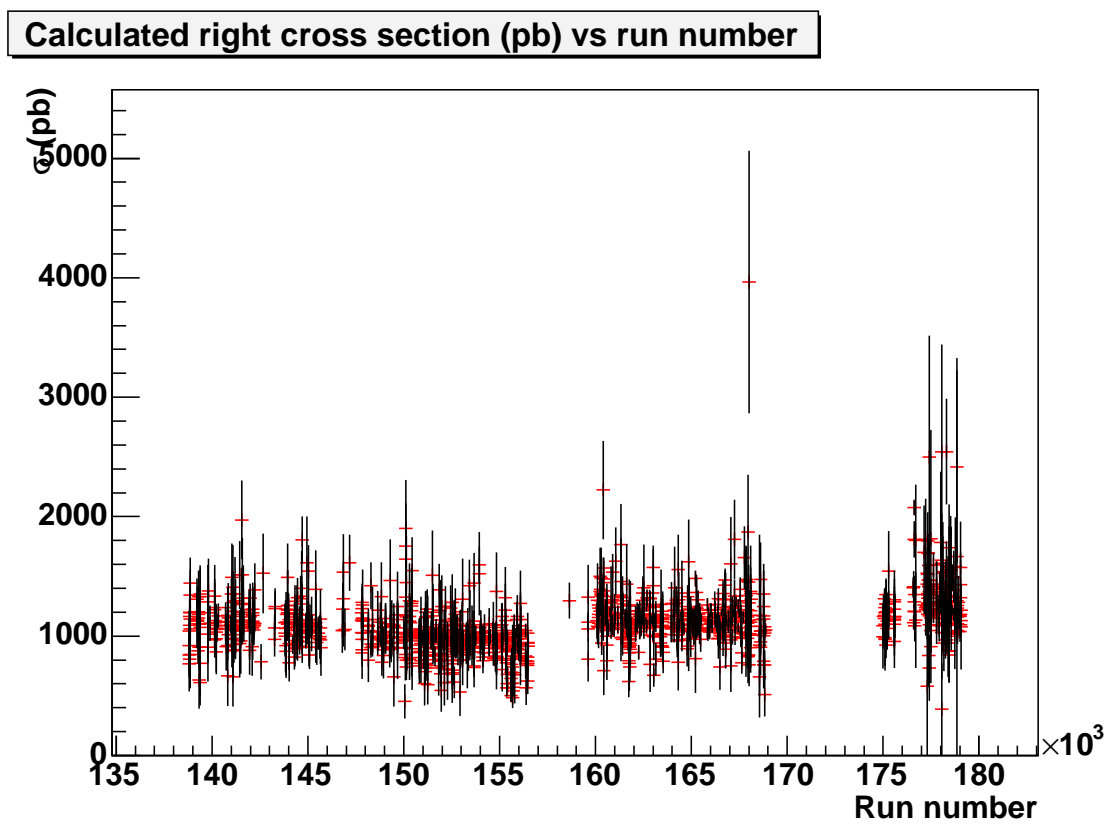

Fig. 4.1: Cross section vs run number for the MET35 trigger. The plot includes a number of cuts to pre-select the data.

\subsubsection{Pre-selection Cuts}

Events were pre-selected using the following basic criteria:

- The presence of a reconstructed primary vertex with $z$-component, $V_{z}$, in the region $\left|V_{z}\right|<$ $60 \mathrm{~cm}$. Events where no vertex is found are excluded.

- At least three jets with transverse energy, $E_{T}^{\text {jet }}$, above $25 \mathrm{GeV}$ and pseudorapidity in the range $\left|\eta^{\text {jet }}\right|<2$.0. Jets are reconstructed using the CDF JETCLU algorithm with a radius $\mathrm{R}=0.7$ and energies corrected for the detector effects and multiple interaction contributions. These corrections change the jet transverse energies between $10 \%$ and $30 \%$ depending on the pseudorapidity. The resolution is $\sim 15 \%$ and improves with the $E_{T}$ of the jet. Systematic uncertainties associated are of the order of $2 \%-3 \%$.

- At least one of the three leading jets is required to be central with a pseudorapidity in the region $\left|\eta^{\text {jet }}\right|<1.1$.

- $\mathbb{E}_{T}>70 \mathrm{GeV}$, where $\mathbb{E}_{T}$ is computed from the energy depositions in the calorimeter towers and the threshold is dictated by the trigger (see Appendix B). Mathematically the $\mathscr{E}_{T}$ is calculated from the vectorial sum of the transverse energies deposited in the different 
calorimeter towers:

$$
\vec{E}_{T}^{\text {raw }}=-\sum_{i}\left(E_{i} \sin \theta_{i}\right) \vec{n}_{i}
$$

where $\vec{n}_{i}$ is the normalised vector that points to the tower from the position of the primary vertex found.

The $\mathbb{E}_{T}$, as measured in the calorimeter, is re-computed using average-corrected jet transverse energies for all the jets in the event. This is expressed with the following formula:

$$
\mathbb{E}_{T}=\mathscr{E}_{T}{ }^{\mathrm{raw}}-\sum_{i=1}^{N_{\mathrm{jets}}} E_{T_{i}}^{\mathrm{uncorr}}+\sum_{i=1}^{N_{\mathrm{jets}}} E_{T_{i}}^{\text {corr }} .
$$

The following requirements were also added in order to remove contributions from beam-halo, beam-gas and cosmic rays. These cuts remove events with insufficient electromagnetic energy deposition in the calorimeter or insufficient tracking activity, inconsistent with jets of hadrons coming from the interaction point (see Appendix C).

- Averaged electromagnetic fraction of jets, defined as EEMF $=\frac{\sum_{\text {jets }} E_{T}^{\text {jet }} \cdot f_{\text {emf }}^{\text {jet }}}{\sum_{\text {jets }} E_{T}^{\text {jet }}}$, greater than 0.15 , where the sum runs over the three leading jets in the event.

- Averaged jet charge fraction, ECHF $\geq 0.15$. The quantity ECHF is defined for central jets $\left(\left|\eta^{\mathrm{jet}}\right|<1.1\right)$ as the averaged ratio between the momentum of the jet, as computed using tracks, and the jet transverse energy:

$$
\mathrm{ECHF}=\frac{1}{N_{\text {jets }}} \sum_{\text {jets }} \frac{\sum_{\text {tracks }} P_{T}^{\text {track }}}{E_{T}^{\text {jet }}},
$$

where only tracks in a cone of radius 0.4 around the jet's direction, and passing the following quality cuts are considered:

$-\left|z-z_{0}\right|<2 \mathrm{~cm}$

$-0.3<p_{T}<500 \mathrm{GeV} / \mathrm{c}$,

$-|\eta|<1.5$,

$-d_{0}<2 \mathrm{~cm}$,

- Number of axial + stereo hits $>20$.

Above, $\left|z-z_{0}\right|$ is the difference in the z-direction between the track and the vertex, and $d_{0}$ is the impact parameter of the track. These cuts are defined after a track validation analysis, using $Z \rightarrow \mu \mu$, presented in Appendix D.

After applying these pre-selection (or basic) cuts, the data sample is cleaner and the $\mathscr{E}_{T}$ spectrum changed significantly as shown in Fig. 4.2. 


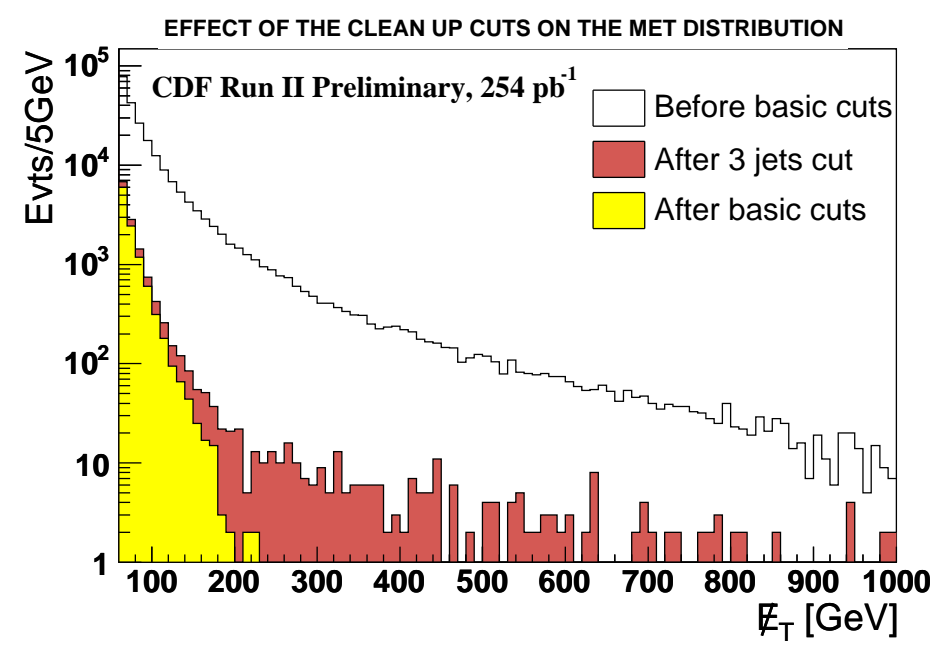

Fig. 4.2: $\not_{T}$ spectrum before and after applying the pre-selection (or basic) cuts.

\subsection{Signal Generation and Normalisation}

In this thesis, the different production channels considered are grouped in four main processes denoted as: $g g$ for $\tilde{g} \tilde{g}$ production; $s g$ for $\tilde{q} \tilde{g}$ (and c.c.) production; $s s$ for $\tilde{q} \tilde{q}$ (and c.c.) production; and $s b$ for $\overline{\tilde{q} q}$ production.

The signal generation and simulation for different squark and gluino masses translate into a substantial amount of CPU time. This analysis was limited to the mSUGRA scenario with the following parameters: $A_{0}=0, \operatorname{sign}(\mu)=-1$ and $\tan \beta=5$. This set of parameters was chosen coherently with other SUSY analyses in CDF and to facilitate the comparison of Run I and Run II results.

PROSPINO [73] was used to calculate the next-to-leading order (NLO) cross sections for squark and gluino production at the Tevatron. In this program, the theoretical calculations are performed using five flavours, assumed to be almost degenerate in mass. In this analysis, processes involving sbottom and/or stop production in the 2-to-2 hard process were excluded, since sbottom and stop masses are significantly smaller than the rest of the squarks, strongly dependent on the mixing, and would dominate the final-state topologies.

The gluino-squark mass plane is then scanned via variations of $m_{0}$ and $m_{1 / 2}$ parameters. Two different leading-order Monte Carlo programs, ISAJET [71] and PYTHIA [72], were initially considered to generate the mSUGRA points. Both matrix elements in ISAJET and PYTHIA give the same prediction for masses and cross sections and PYTHIA Monte Carlo was finally chosen since the initial- and final-state gluon radiation in ISAJET is poorly modelled (see Appendix E). The generation also used CTEQ5L PDFs, initial-state gluon radiation and underlying event set- 
tings as determined by Tune $\mathrm{A}$, and $\Lambda_{Q C D}=146 \mathrm{MeV}^{1}$.

As it can be seen in Fig. 4.3, more than 100 different points were generated in a grid with different squark and gluino masses. This grid of points was chosen to overlap Run I limits and to expand up to approximately $500 \mathrm{GeV} / \mathrm{c}^{2}$ (see also Fig. 4.4). Additional PYTHIA samples were generated with enhanced and reduced initial- and final-state gluon radiation to determine the systematic uncertainty due to the parton shower modeling in the Monte Carlo.

PYTHIA generation points

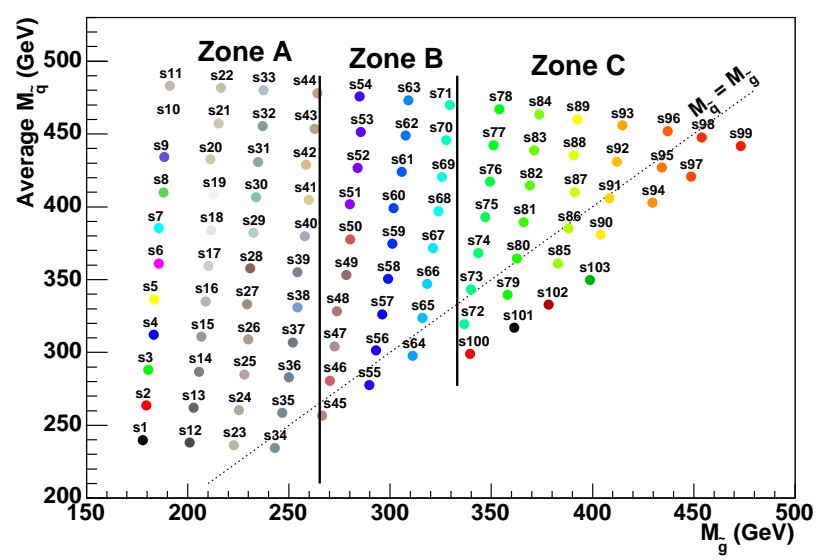

Fig. 4.3: mSUGRA points generated with PYTHIA. The y-axis is the average mass of the 8 squarks of the first two generations. The x-axis corresponds to the mass of the gluino. 15,000 events were generated for each point. The plot is divided into the three zones obtained from the optimisation study.

Using PROSPINO, the cross section for each of the subprocesses $\sigma_{\mathrm{NLO}}^{i}$ with $i=g g, s g, s s$, and $s b$ is calculated. Then, with the condition:

$$
\sigma_{\mathrm{NLO}}=\sum_{i} \sigma_{\mathrm{NLO}}^{i}
$$

the value of the relative cross sections $\left(k_{i} \equiv \sigma_{\mathrm{NLO}}^{i} / \sigma_{\mathrm{NLO}}\right)$ is used to obtain the correspondent relative values on the number of events. If the efficiency for each subprocess after a certain number of cuts is $\varepsilon_{i} \equiv N_{i} / N_{i}^{0}$, where $N_{i}$ is the number of signal events for the subprocess $i$ surviving the cuts and $N_{i}^{0}$ is the initial number of events for the same subprocess, then the total efficiency for one of the points in the grid is:

$$
\varepsilon=\sum_{i} \varepsilon_{i} \cdot k_{i}, \quad i=\mathrm{g} g, s g, s s, s b
$$

Following this procedure, the number of expected events for a particular signal point can be calculated using NLO estimations. The default value of the NLO renormalisation scale is

\footnotetext{
${ }^{1}$ This follows the standards suggested by the CDF Top and Higgs Working Groups.
} 


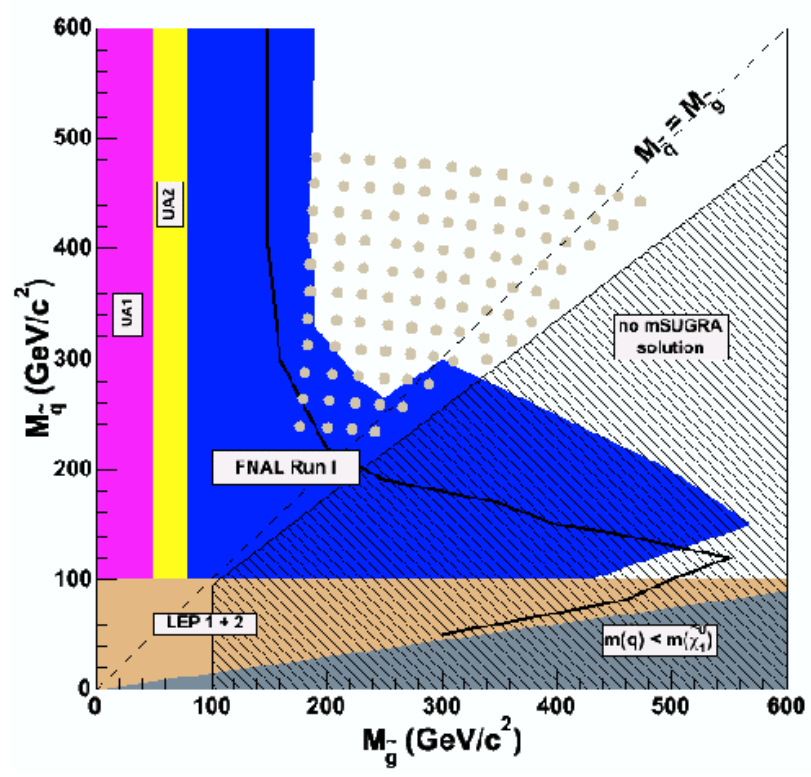

Fig. 4.4: mSUGRA points generated with PYTHIA in the context of the limits found by previous analyses. The $y$-axis is the average mass of the 8 squarks of the first two generations and the $x$-axis corresponds to the mass of the gluino.

set to $\mu=\mathrm{M}_{\tilde{\mathrm{g}}}$ for gluino-gluino (gg) production processes; $\mu=0.5\left[\mathrm{M}_{\tilde{\mathrm{g}}}+\overline{\mathrm{M}}_{\tilde{\mathrm{q}}}\right]$ for squark-gluino (sg) production processes; $\mu=\overline{\mathrm{M}}_{\tilde{\mathrm{q}}}$ for squark-squark (ss) or antisquark-antisquark production processes and $\mu=\overline{\mathrm{M}}_{\tilde{q}}$ for squark-antisquark (sb) production processes. Here, $\overline{\mathrm{M}}_{\tilde{q}}$ is the average of all eight squark masses (two first generations) considered.

\subsection{Background Processes}

The SM background in this analysis is dominated by QCD multijet processes where the observed $\mathbb{E}_{T}$ comes from an inadequate determination of the jet transverse energies. In addition, there are contributions from $\mathrm{Z}$ and $\mathrm{W}$ production in association with jets, top production and diboson production. In particular, the contribution from $Z+3$ jets production, where the $Z$ decays into neutrinos, constitutes an irreducible background to the mSUGRA signal. A list of the most relevant background processes is given in Tab. 4.2.

The different boson + jets and diboson samples were normalised using LO-to-NLO $k$-factors determined by MCFM $^{2}$ [74]. The default value of the renormalisation scale in MCFM was set to $\mu=\mathrm{M}_{W}\left(\mu=\mathrm{M}_{Z}\right)$ in the case of $\mathrm{W}(\mathrm{Z})$ processes. To normalise the $t \bar{t}$ production, the NLO

\footnotetext{
${ }^{2}$ MCFM provides $k$-factor upto two partons in the final state. However, as shown by separate measurements at D $\emptyset$, the use of $k$-factors for three partons is a reasonable approximation.
} 


\begin{tabular}{|c|c|c|}
\hline \hline Sample & Sample Lumi (pb-1) & $k$-factor (NLO/LO) \\
\hline \hline$Z \rightarrow v v+3$ jets & $1.66 \times 10^{3}$ & 1.13 \\
\hline$Z / \gamma * \rightarrow e e+2$ jets & $1.16 \times 10^{4}$ & 1.18 \\
\hline$Z / \gamma * \rightarrow \mu \mu+2$ jets & $7.76 \times 10^{3}$ & 1.18 \\
\hline$Z / \gamma * \rightarrow \tau \tau+2$ jets & $7.93 \times 10^{3}$ & 1.18 \\
\hline \hline$W \rightarrow e v+3$ jets & $2.62 \times 10^{3}$ & 1.09 \\
\hline$W \rightarrow \mu \nu+3$ jets & $3.21 \times 10^{3}$ & 1.09 \\
\hline$W \rightarrow \tau v+2$ jets & $7.61 \times 10^{2}$ & 1.09 \\
\hline \hline$t \bar{t}($ all decays $)$ & $3.45 \times 10^{4}$ & NLO theory $\sigma$ \\
\hline$W W($ all decays $)$ & $4.17 \times 10^{3}$ & 1.41 \\
\hline \hline QCD $60<\hat{p}_{T}<90 \mathrm{GeV}$ & $4.36 \times 10^{1}$ & from data \\
\hline QCD $90<\hat{p}_{T}<120 \mathrm{GeV}$ & $7.64 \times 10^{2}$ & from data \\
\hline QCD $120<\hat{p}_{T}<150 \mathrm{GeV}$ & $9.58 \times 10^{2}$ & from data \\
\hline QCD $150<\hat{p}_{T}<200 \mathrm{GeV}$ & $1.54 \times 10^{3}$ & from data \\
\hline QCD $200<\hat{p}_{T}<300 \mathrm{GeV}$ & $7.03 \times 10^{3}$ & from data \\
\hline QCD $300<\hat{p}_{T}<400 \mathrm{GeV}$ & $1.65 \times 10^{5}$ & from data \\
\hline QCD $400<\hat{p}_{T}<500 \mathrm{GeV}$ & $3.07 \times 10^{6}$ & from data \\
\hline QCD $\hat{p}_{T}>500 \mathrm{GeV}$ & $5.76 \times 10^{7}$ & from data \\
\hline \hline
\end{tabular}

Tab. 4.2: List of the SM background processes considered for this analysis and the normalisation to NLO ( $k$-factor).

theoretical cross section was used [75]. Dedicated studies were carried out to determine the normalisation of the QCD samples from the data and the minimum $\hat{\mathrm{p}}_{\mathrm{T}}$ that contributes to the $\mathbb{E}_{T}$ trigger data, since it is virtually impossible to generate sufficient Monte Carlo statistics for an arbitrarily low $\hat{\mathrm{p}}_{\mathrm{T}}$ threshold. Both studies are described in Appendix F. It was found that the ratio data/MC indicates that no $k$-factor different than 1.0 is necessary and that the minimum $\hat{\mathrm{p}}_{\mathrm{T}}$ necessary is $90 \mathrm{GeV} / \mathrm{c}$.

\subsection{Selection Cuts}

In addition to the pre-selection criteria described in Section 4.1.2, a number of selection cuts are applied to significantly reduce the different SM backgrounds.

\subsubsection{Multijet Background (QCD) Rejection}

The production of multiple jet events coming from QCD processes has a huge cross section. A priori, these events should not be characterised for having much $\mathbb{E}_{T}$. However, large missing 
energy may arise from the mismeasurement of the jet energy due to cracks and/or other detector effects. When a jet is partially reconstructed in the detector, it is expected that the azimuthal direction of the resulting $\mathbb{E}_{T}$ be aligned to the jet in the transverse plane. Fig. 4.5 shows the azimuthal angle between the $\mathbb{E}_{T}$ and each of the three leading jets. As expected, a peak at $\Delta \phi=0$ is observed. For comparison, Fig. 4.6 shows a similar plot for three representative mSUGRA signal points where no peak is observed.
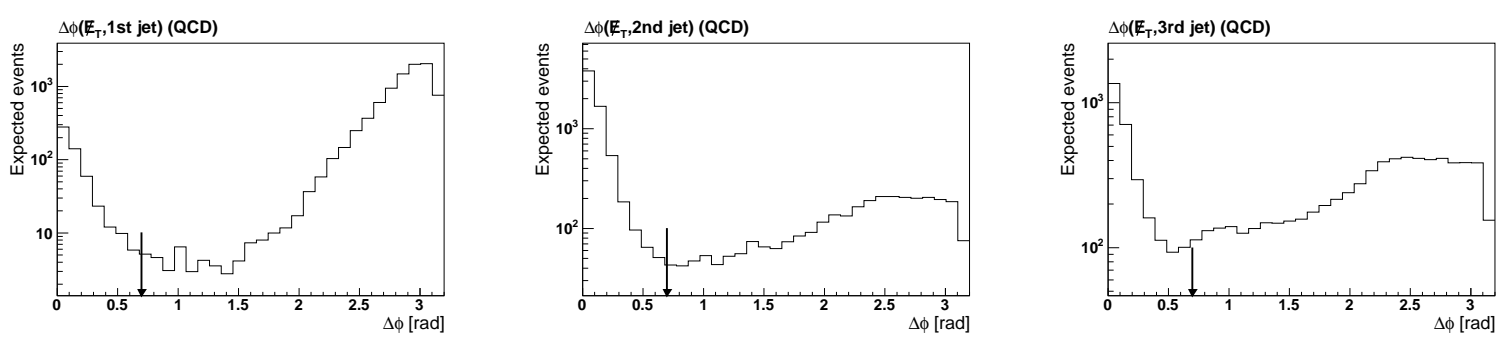

Fig. 4.5: $\Delta \phi\left(\not_{T}\right.$, jets $)$ distributions for the three leading jets of the QCD multijet sample. The peak at zero comes from events in which one of the jets is mismeasured resulting in a $\mathbb{E}_{T}$ aligned with the jet. The arrow indicates the value of the cut.
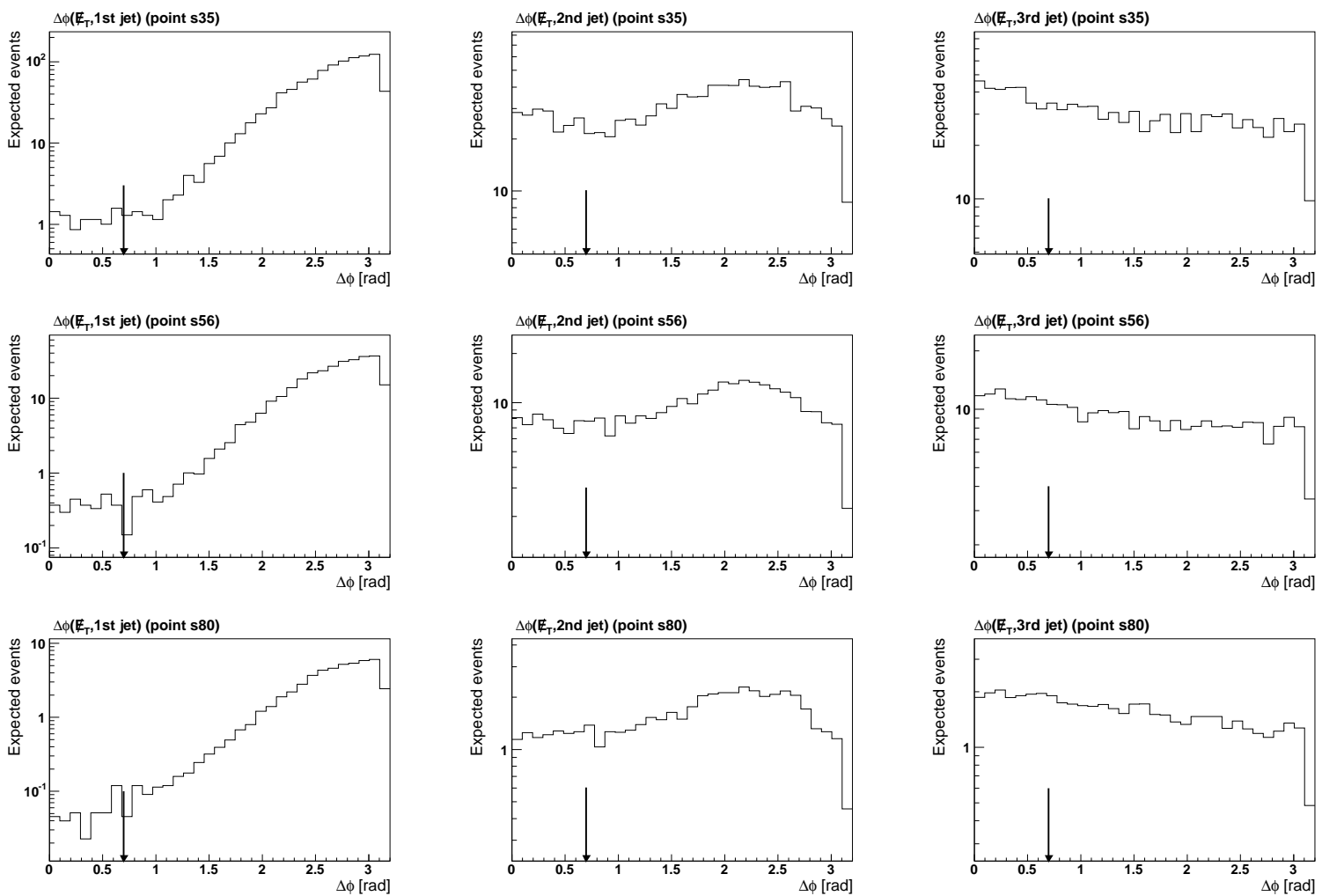

Fig. 4.6: $\Delta \phi\left(\mathbb{Z}_{T}\right.$, jets $)$ distributions for the three leading jets of the representative mSUGRA point: $s 35$ (top), s56 (middle), s80 (bottom). The $\not_{T}$ in signal events points in a direction away from the jets. The arrow indicates the value of the cut. 
Therefore, to remove the contribution from multijet QCD background we require the azimuthal distance between $\mathbb{E}_{T}$ and the direction of each of the three leading jets to be larger than the cone size of the jet.

$$
\Delta \phi\left(\mathbb{E}_{T}, \mathrm{jet}\right)>0.7
$$

\subsubsection{Electron Rejection}

$\mathrm{W}$ and $\mathrm{Z}$ bosons decay into electrons a fraction of the time. These electrons can be misidentified as jets. To reject this contribution, the electromagnetic fraction (EMF), defined as the ratio between the transverse electromagnetic energy of the jet to the total transverse energy of the jet, measured by the calorimeter, is required to be less than 0.9 .

$$
\mathrm{EMF}_{\text {jets }}<0.9
$$

Fig. 4.7 shows the EMF distributions of the three leading jets for representative mSUGRA points. Similarly, Fig. 4.8 shows the EMF distribution for $W \rightarrow e v$. The peak at one is due to electrons. In the case of the mSUGRA samples, some electrons may also come from semileptonic decays in the development of the gluino and squark cascades.

\subsubsection{Muon Rejection}

$\mathrm{W}$ and $\mathrm{Z}$ bosons can also decay into muons. These muons, if not detected, can produce large $\mathbb{E}_{T}$ in the event becoming a significant background in the analysis. Muons can be identified in the COT as isolated tracks. We define isolation for those tracks with $\mathrm{p}_{\mathrm{T}}>10 \mathrm{GeV} / \mathrm{c}$. A track is considered isolated if the scalar $\mathrm{p}_{\mathrm{T}}$ sum of all additional tracks in a cone of radius, $\mathrm{R}=0.4$, is less than $2 \mathrm{GeV} / \mathrm{c}$, where only tracks as defined in section 4.1.2 are considered.

With the above definitions two different cuts are implemented to reject these specific backgrounds:

- $Z / \gamma^{*} \rightarrow \mu \mu$ : events are rejected if the invariant mass of the two highest isolated tracks falls in the $76<\mathrm{M}_{\mathrm{inv}}<106$ mass window. The invariant mass distribution for the mSUGRA points is shown on the left column of Fig. 4.9. As it can be seen, there are very few signal events which have two or more isolated tracks. Fig. 4.10 shows the distribution for $Z / \gamma^{*} \rightarrow \mu \mu$ and $W \rightarrow \mu \nu$ events. Most of the events from the first one are rejected by this cut.

- $W \rightarrow \mu v$ : events are rejected if the azimuthal angle between the highest isolated track and the $\mathbb{E}_{T}$ is below 0.7. This cut is analogous to the cut for QCD rejection as it eliminates 

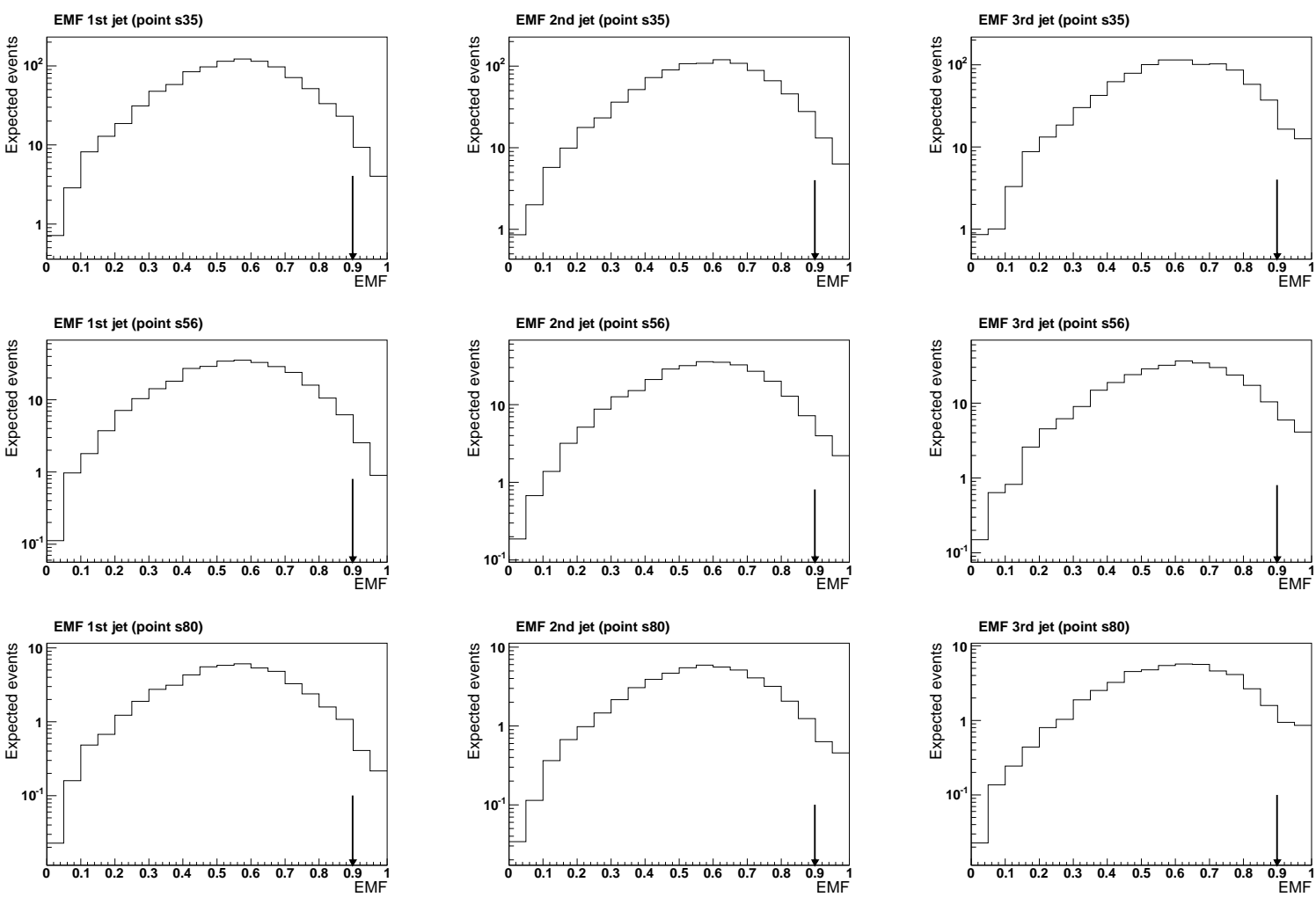

Fig. 4.7: EMF distributions for the three leading jets of the representative mSUGRA points: $s 35$ (top), s56 (middle), s80 (bottom). The arrow indicates the value of the cut.
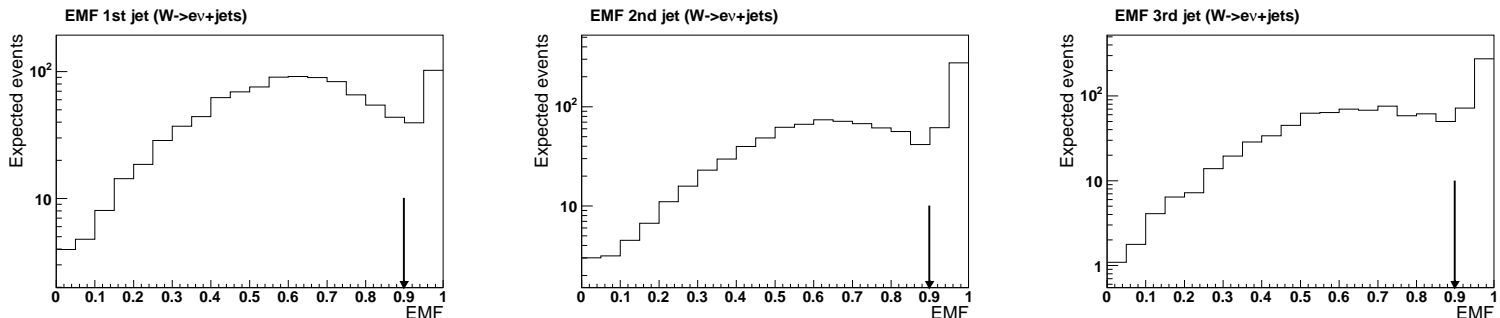

Fig. 4.8: EMF distribution for the leading jets of the $\mathrm{W} \rightarrow \mathrm{ev}$ sample. A large fraction of the jets have an EMF close to one. These jets are most likely electrons. The arrow indicates the value of the cut.

events where the $\mathbb{E}_{T}$ is caused by an undetected muon. Fig. 4.11 shows the azimuthal angle distribution between the $\mathscr{E}_{T}$ and the isolated track for $Z / \gamma^{*} \rightarrow \mu \mu$ and $W \rightarrow \mu v$. Both distributions peak at zero due to events in which the $\mathbb{E}_{T}$ is aligned with an isolated track. The right column of Fig. 4.9 shows a similar distribution for the mSUGRA signal points. This cut removes a large fraction of the backgrounds while keeping most of the signal. 

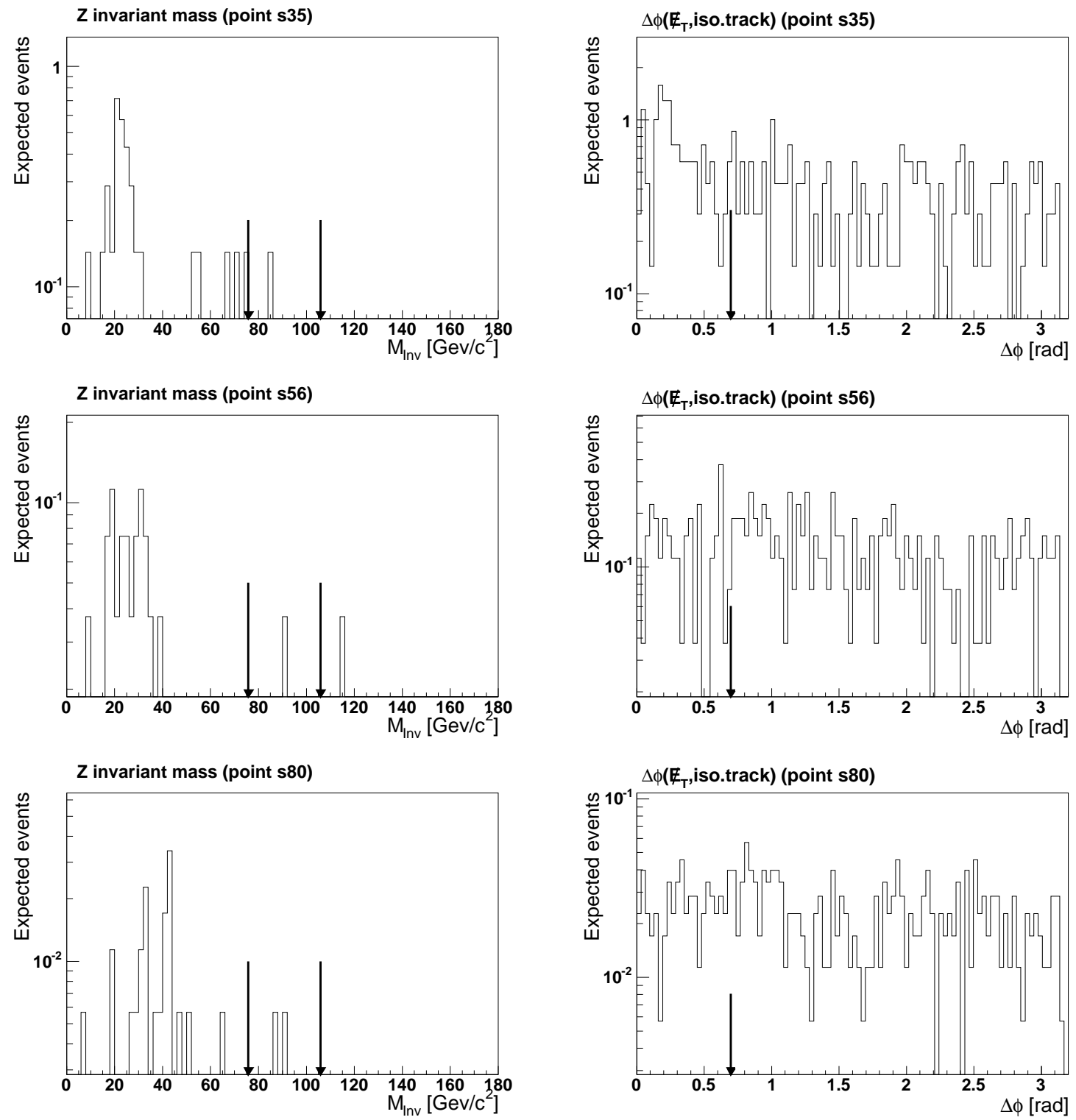

Fig. 4.9: The left column shows the distribution of the invariant mass of the two highest isolated tracks. The right column shows the distribution of the azimuthal angle between the $\mathbb{E}_{T}$ and the highest isolated track. Plots are shown for s35 (top), s56 (middle), and s80 (bottom). The arrows show where the different cuts are placed.

\subsubsection{Further Rejection: Signal vs Background Optimisation}

The previous cuts were introduced to reduce specific background processes. However, the background contribution in general can be further reduced using the fact that squarks and gluinos produce large $\mathscr{E}_{T}$ and have large masses. It is expected that the mSUGRA signal events be characterised by isotropic (spherical) final-state topologies with large amounts of transverse energy measured in the calorimeter. The variables that help on discriminating signal from background at 

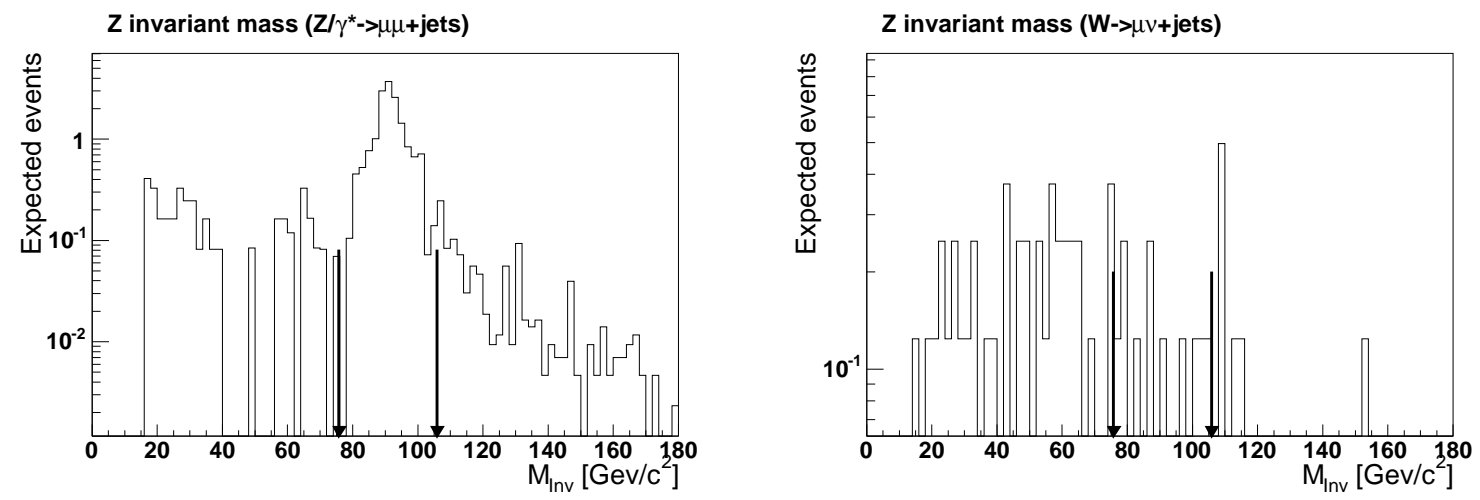

Fig. 4.10: Invariant mass distribution of the two highest isolated tracks in the event. The plot on the left corresponds to $Z / \gamma^{*} \rightarrow \mu \mu$, while the plot on the right corresponds to $W \rightarrow \mu \nu$. This cut does little to reduce the $W \rightarrow \mu \nu$ background since not many of the events have two or more isolated tracks. However, it rejects most of the $Z / \gamma^{*} \rightarrow \mu \mu$ background. The arrows show were the invariant mass window cut is placed.
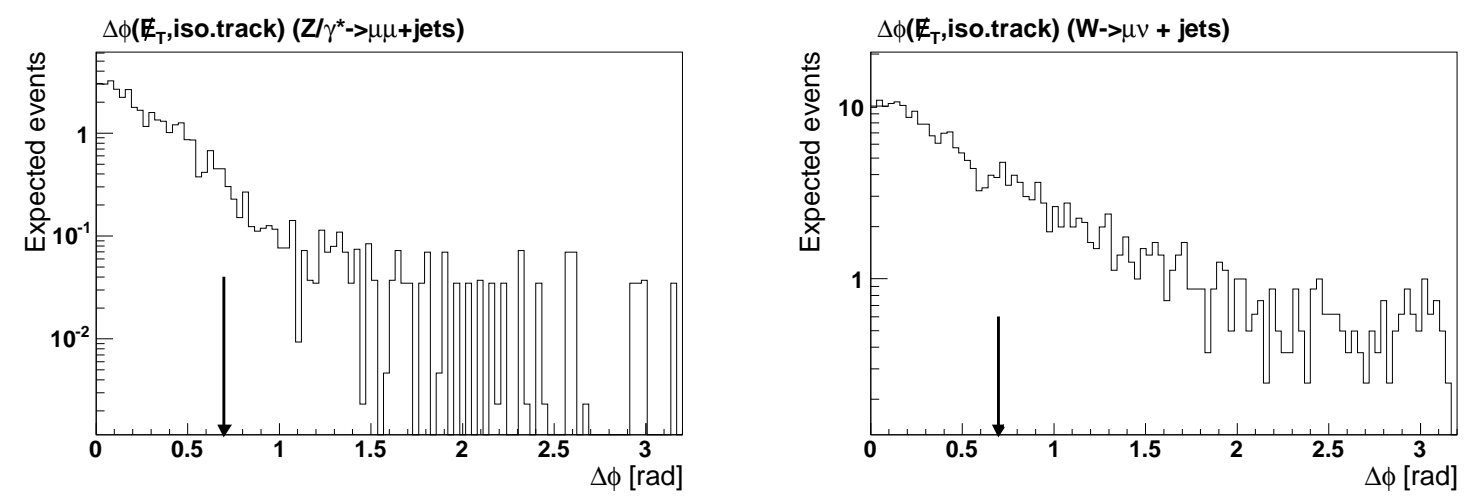

Fig. 4.11: $\Delta \phi$ (track, $\left.\mathbb{E}_{\mathrm{T}}\right)$ distribution for $Z / \gamma^{*} \rightarrow \mu \mu$ events (left) and $W \rightarrow \mu \nu$ events (right). This cut rejects events in which the direction of the $\mathbb{E}_{T}$ is aligned with that of an isolated track. A significant fraction of the backgrounds is rejected by the cut placed where the arrow indicates.

this point are the following:

- $E_{T}^{\text {jet } 1}$ : transverse energy of the leading jet.

- $E_{T}^{\text {jet } 2}$ : transverse energy of the second leading jet.

- $E_{T}^{\text {jet3 }}$ : transverse energy of the third leading jet.

- $\mathrm{H}_{\mathrm{T}}$ : total transverse energy defined as the sum of the transverse energies of the three leading jets $\left(H_{T}=\sum_{\text {jets }}^{3} E_{T}^{\text {jet }}\right)$.

- $\mathbb{E}_{T}$ : missing transverse energy corrected as explained in Section 4.1.2. 
A careful MC study was performed to set the optimal values for these variables and maximise their ability to separate the mSUGRA signal from the background. This ability is quantified by $\mathrm{S} / \sqrt{\mathrm{B}}$, where $\mathrm{S}$ denotes the expected number of signal events and $\sqrt{\mathrm{B}}$ is the statistical uncertainty on the Standard Model background. Due to the complexity of optimising 103 points of signal with five variables that are correlated, a step-by-step procedure was considered:

- First, the number of points in Fig. 4.3 was reduced and only the points with similar squark mass and increasing gluino mass (row points 31-91) together with a set of points with $\mathrm{M}_{\tilde{\mathrm{q}}} \approx \mathrm{M}_{\tilde{\mathrm{g}}}$ (diagonal points 23-91) were selected in this study. It is noticeable that the difference between points belonging to the same column, or same gluino mass, is less significant. At the bottom of a column, squarks and gluinos have similar masses, and therefore they tend to be produced with similar probability. As the mass of the squark increases (moving up in the column), the squark cross section becomes smaller. Thus, at the top of the column most of the events tend to come from gluinos, whose mass has not changed.

All optimisation plots were done with these limited set of mSUGRA points, with the aim to determine the different cuts defining the minimum number of zones into which the mSUGRA mass plane can be divided.

- Fig. 4.12 and Fig. 4.13 show the $\mathrm{S} / \sqrt{\mathrm{B}}$ distributions for the points with $\mathrm{M}_{\tilde{\mathrm{q}}} \approx \mathrm{M}_{\tilde{\mathrm{g}}}$ (diagonal), and the points with similar squark masses (row), respectively. Each triangle in each of the plots corresponds to a different cut on $\mathrm{H}_{\mathrm{T}}$. From these plots, three different regions are defined. In region $\mathrm{A}$, the $\mathrm{H}_{\mathrm{T}}$ cut is set to $255 \mathrm{GeV}$. Region $\mathrm{B}$ has a $\mathrm{H}_{\mathrm{T}}$ cut of $330 \mathrm{GeV}$. Finally, region $\mathrm{C}$ has a cut of $355 \mathrm{GeV}$. All of these cuts are directly extracted from the maximum of the $S / \sqrt{B}$ distributions.

Fig. 4.14 and Fig. 4.15 show the $\mathrm{H}_{\mathrm{T}}$ distributions for the points with $\mathrm{M}_{\tilde{\mathrm{q}}} \approx \mathrm{M}_{\tilde{\mathrm{g}}}$ (diagonal), and similar squark mass (row), respectively. When comparing the distributions, the difference between two points with similar gluino mass and different squark masses (points 23-31, 35-42, 46-52, etc) is negligible compared to the difference between two points with similar squark masses and different gluino masses.

- The $\mathrm{H}_{\mathrm{T}}$ cut is applied and now a similar study is performed with the $\mathbb{E}_{T}$ variable. Fig. 4.16 and Fig. 4.17 show the $S / \sqrt{B}$ distribution for the points along the diagonal and the row. From these plots, a $E_{T}$ cut of $75 \mathrm{GeV}$ for region $\mathrm{A}$ is assigned. The cuts for regions B and $\mathrm{C}$ are chosen to be $100 \mathrm{GeV}$ and $130 \mathrm{GeV}$, respectively. Fig. 4.18 shows the $\mathscr{E}_{T}$ distribution for the points along the diagonal. Similarly, Fig. 4.19 shows the $\mathbb{E}_{T}$ distribution for the points along the row.

- After applying the $\mathrm{H}_{\mathrm{T}}$ and $\mathbb{E}_{T}$ cuts, the $\mathrm{E}_{\mathrm{T}}$ of the jets have less discriminating power. As an example, Fig. 4.20 shows the $\mathrm{S} / \sqrt{\mathrm{B}}$ distribution of the leading jet for points along the 
diagonal. The distribution is flat until it starts to drop, which implies that signal and background have the same shape. Therefore, the cut on this variables is chosen so that the signal acceptance remains high and the low-end tails of the jet's $\mathrm{E}_{\mathrm{T}}$ distributions are removed. This can be seen in Fig. 4.21 through Fig. 4.23, where the arrows show the placement of the cut for the different regions. The only cut applied to the third jet transverse energy is the one from the pre-selection $(25 \mathrm{GeV})$.
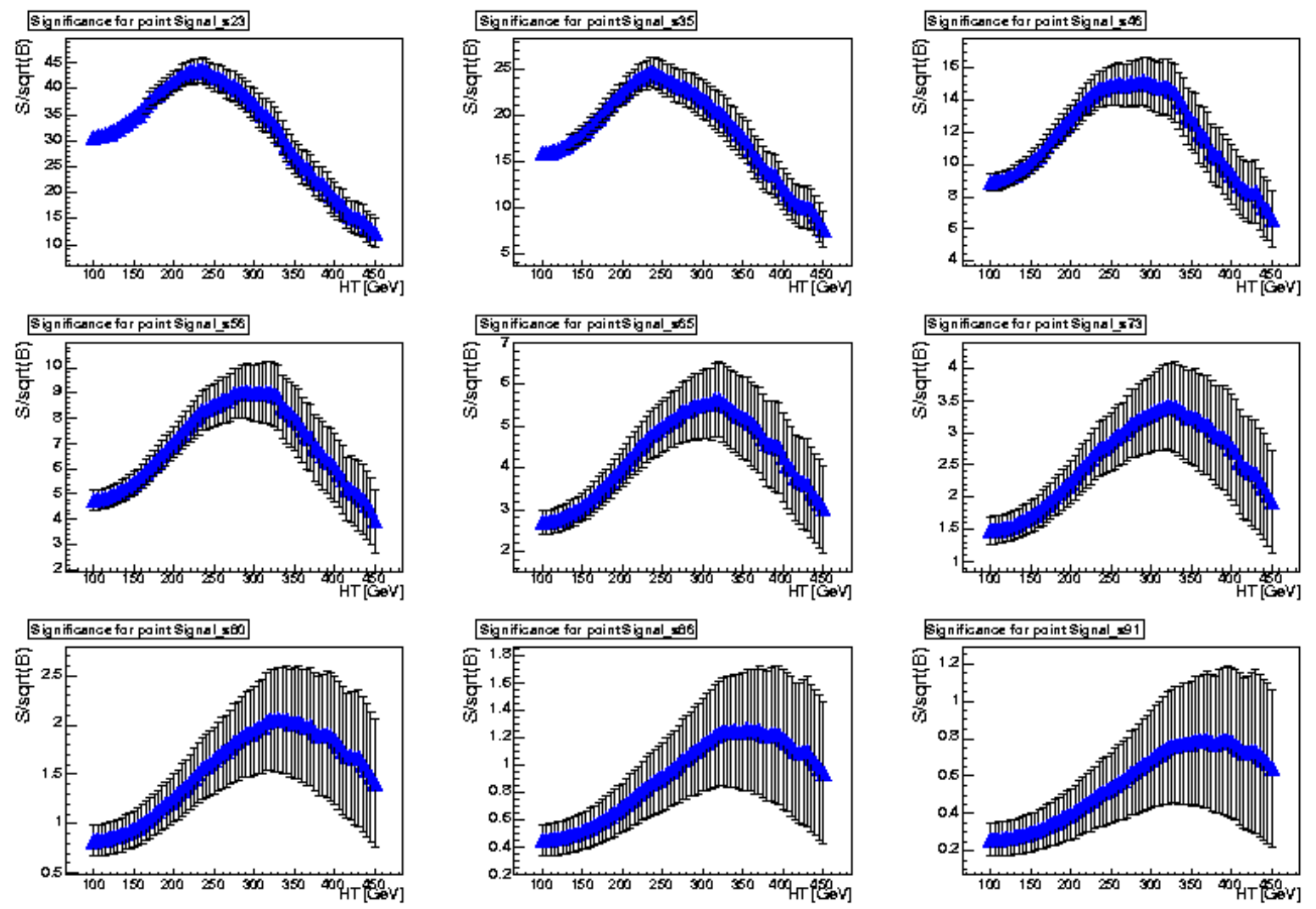

Fig. 4.12: $\mathrm{S} / \sqrt{\mathrm{B}}$ distribution for $\mathrm{H}_{\mathrm{T}}$ of the mSUGRA points along the diagonal.

Different ordering for the applied cuts was also studied and the one which maximised the $S / \sqrt{B}$ was chosen.

In conclusion, the entire generated mSUGRA plane was finally divided into three distinct regions as a function of the gluino mass, as seen in Fig. 4.3. The value of the different thresholds which defined the three different signal regions is shown in Tab. 4.3 and the efficiencies for each of the cuts applied in the analysis (pre-selection and optimised cuts) for three representative mSUGRA points, together with the number of expected events after all cuts, are given in Tab. 4.4. Only statistical uncertainties are considered at this point. 

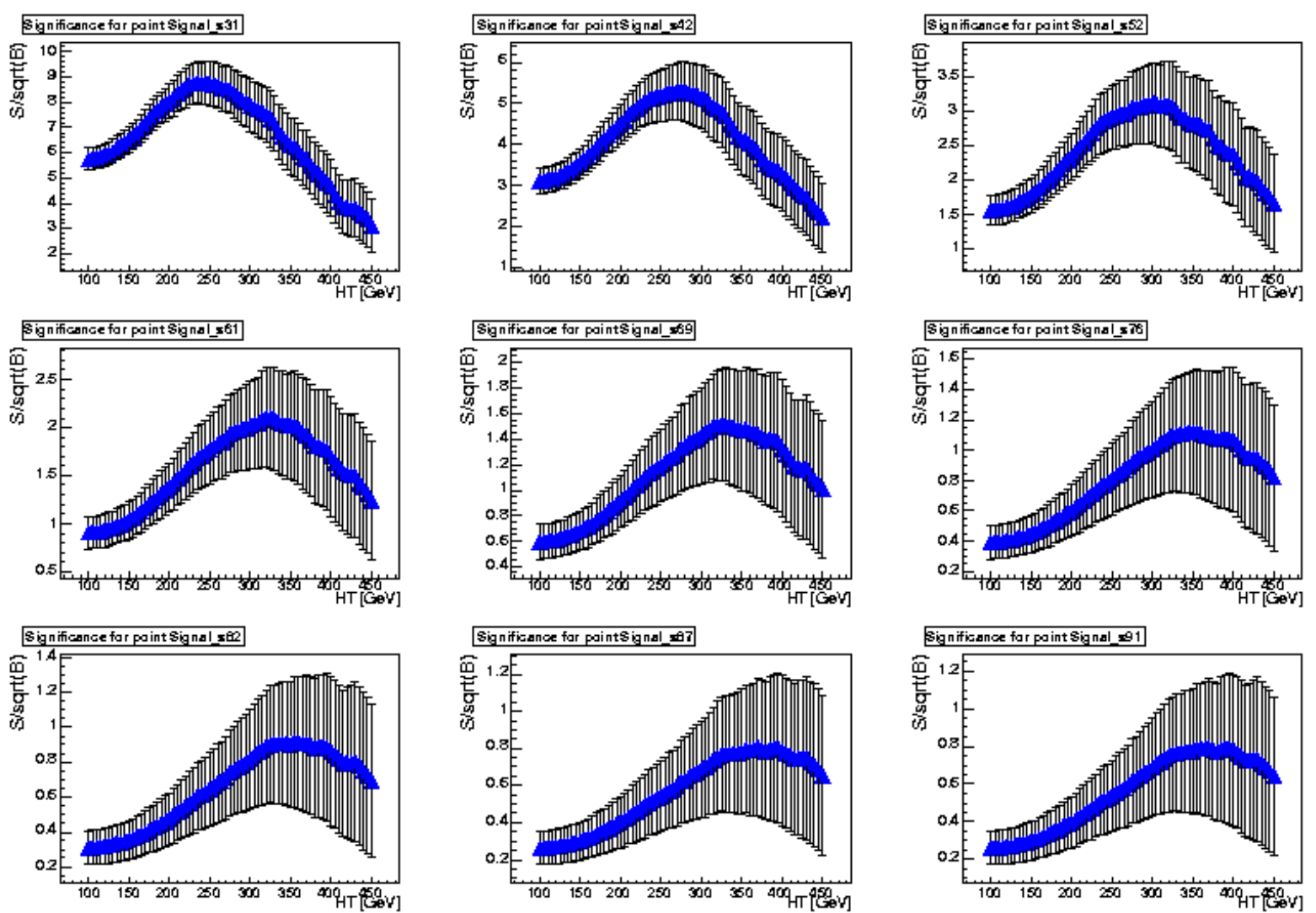

Fig. 4.13: $\mathrm{S} / \sqrt{\mathrm{B}}$ distributions for $\mathrm{H}_{\mathrm{T}}$ of the mSUGRA points along the row.

\begin{tabular}{|l|c|c|c|c|c|}
\hline \hline & $E_{T}(\mathrm{GeV})$ & $\mathrm{H}_{\mathrm{T}}(\mathrm{GeV})$ & $\mathrm{E}_{\mathrm{T}}^{\mathrm{jet} 1}(\mathrm{GeV})$ & $\mathrm{E}_{\mathrm{T}}^{\text {jet2 }}(\mathrm{GeV})$ & $\mathrm{E}_{\mathrm{T}}^{\text {jet3 }}(\mathrm{GeV})$ \\
\hline Region A & 75 & 230 & 95 & 55 & 25 \\
\hline Region B & 90 & 280 & 120 & 70 & 25 \\
\hline Region C & 120 & 330 & 140 & 100 & 25 \\
\hline \hline
\end{tabular}

Tab. 4.3: Cut thresholds for the three different regions in which the signal plane is divided.

\subsection{Signal Studies}

In order to better understand the signal behaviour as a function of the different gluino and squark masses, the variation of different quantities such as efficiencies, significances and relative contributions to the production subprocesses, were studied along columns (constant $M_{\tilde{g}}$ ), along rows (constant $\left.M_{\tilde{q}}\right)$ and along the diagonal $\left(M_{\tilde{g}} \approx M_{\tilde{q}}\right)$ in the signal plane. The relative contributions for the different production subprocesses, gluino-gluino (gg), squark-gluino (sg) and squark-squark 

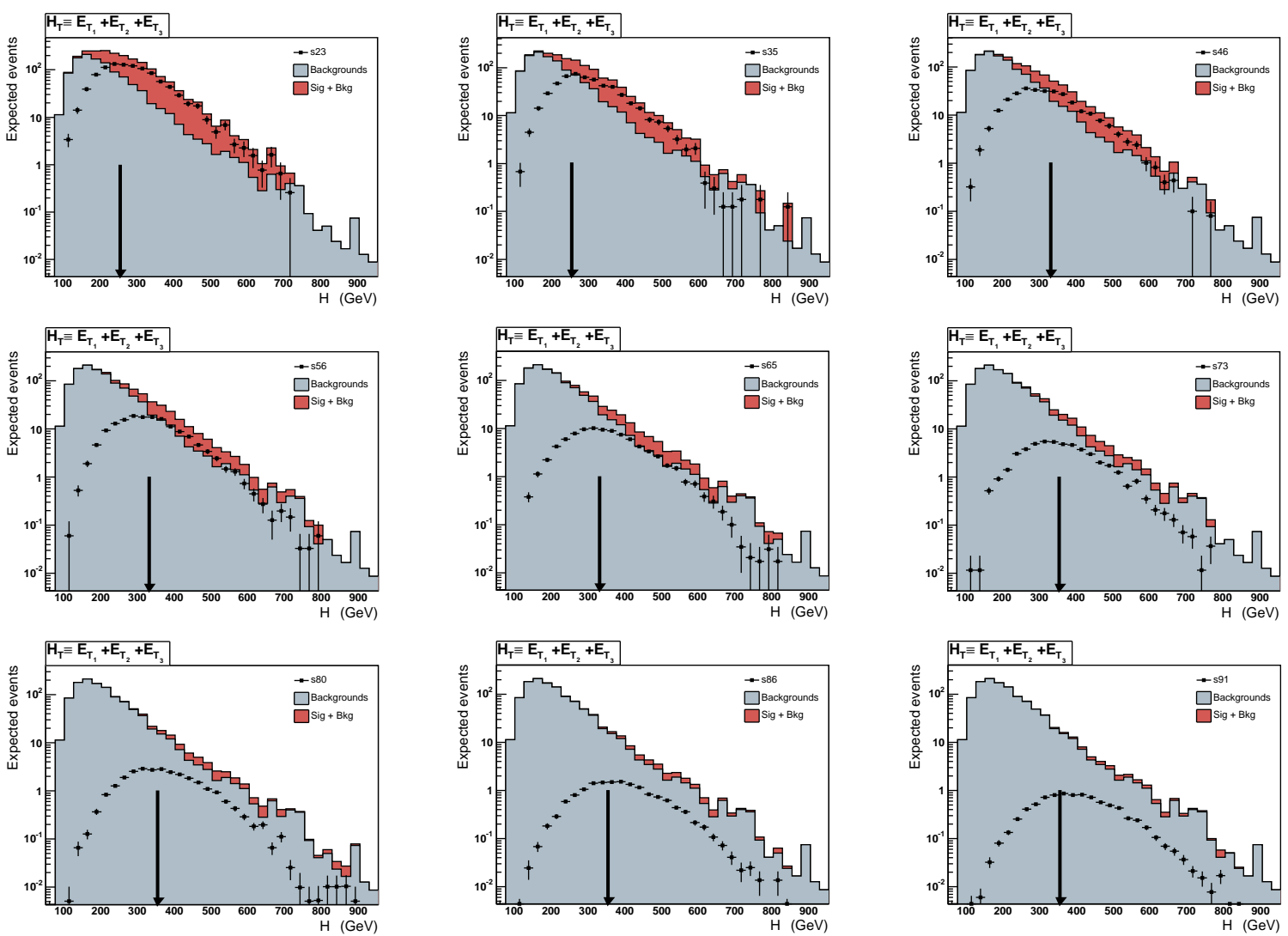

Fig. 4.14: $\mathrm{H}_{\mathrm{T}}$ distributions for all the points in the diagonal $\left(\mathrm{M}_{\tilde{\mathrm{q}}} \approx \mathrm{M}_{\tilde{\mathrm{g}}}\right)$. The arrow shows where the cut is placed for each different zone. The first two plots correspond to zone A (points s23, and s35), the next four plots correspond to zone B (points s46, s56, and s65), and the last three plots correspond to zone C (points s73, s80, s86, and s91).

(or with anti-squarks) (ss) ${ }^{3}$ were studied before any cut and after all the analysis cuts. The results and their interpretation follows:

- Study along column $\mathbf{s 1}\left(M_{\tilde{g}} \approx 185 \mathrm{GeV} / \mathrm{c}^{2}\right)$ In Fig. 4.24 the relative contributions of the different production subprocesses is shown together with their efficiencies and significances. Before the cuts, the gg contribution is steadily increasing as long as the ss

\footnotetext{
${ }^{3}$ In this section, for simplicity, the (ss) refers to the sum of the squarks and anti-squarks production processes.
} 

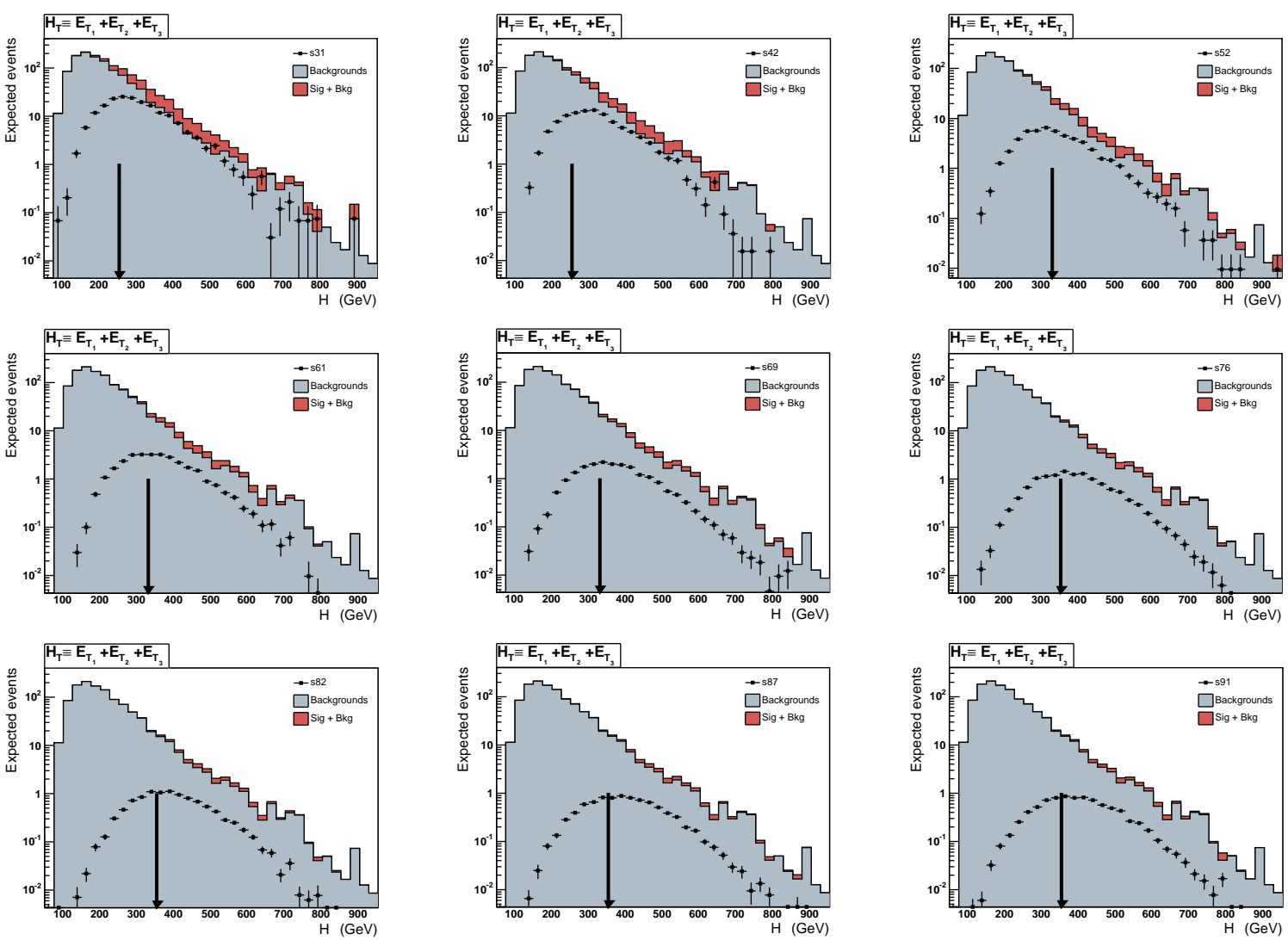

Fig. 4.15: $\mathrm{H}_{\mathrm{T}}$ distributions for all the points in the chosen row (gluino mass increases as the point number increases). The arrow shows where the cut is placed for each different zone. The first two plots correspond to zone A (points s31, and s42), the next four histograms correspond to zone B (points s52, s61, and s69), and the last three plots correspond to zone $\mathrm{C}$ (points $\mathrm{s} 76, \mathrm{~s} 82, \mathrm{~s} 87$, and s91).

contribution is being suppressed due to the increasing squark mass. After the cuts, the gg contribution is slightly reduced. The most probable explanation for this effect is the fact that the jets coming from the gluino tend to be softer than a single jet produced from a squark of a similar mass. Therefore, it is the minimum threshold condition of the first and the second jet (in zone A: $E_{T}^{\text {jet } 1} \geq 95 \mathrm{GeV}$ and $E_{T}^{\text {jet } 1} \geq 55 \mathrm{GeV}$ ) which are reducing the $\mathrm{gg}$ contribution.

- Study along diagonal $\mathbf{s 3 4}\left(M_{\tilde{g}} \approx M_{\tilde{q}}\right)$ Same quantities as in the previous case are shown in Fig. 4.25, but now along the diagonal where the mass of the squark is comparable to the gluino mass. The two vertical lines separate the three optimised regions. As expected, 

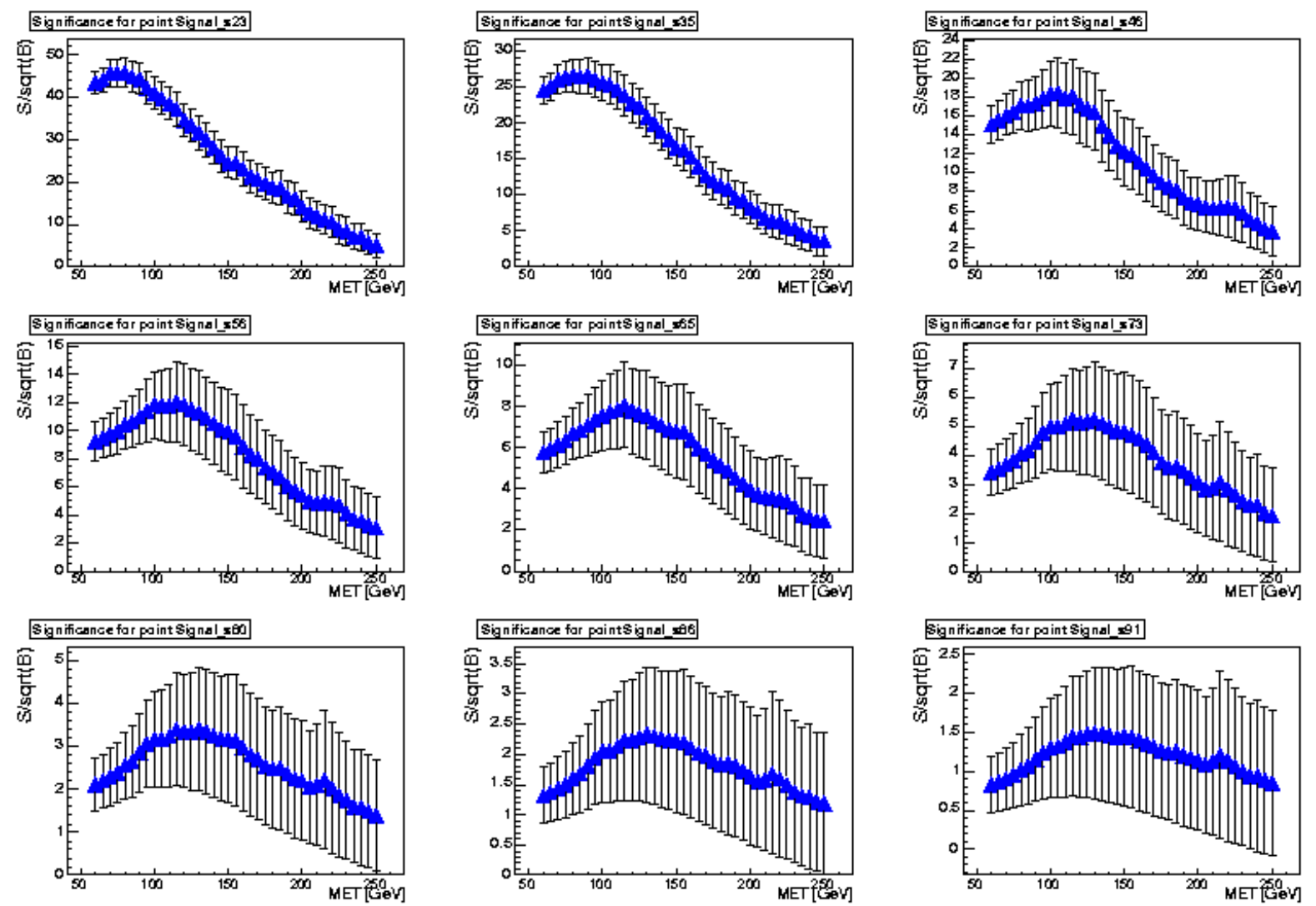

Fig. 4.16: $\mathrm{S} / \sqrt{\mathrm{B}}$ distribution for $\not_{T}$ of the mSUGRA points along the diagonal.

the three different signal production processes keep their relative contribution along the diagonal, even after the analysis cuts. The efficiencies increase along the diagonal and the significances drop with the increasing mass, as expected, but the different optimisations per each region helps on reducing the falling rates.

- Study along row $\mathbf{s 6}\left(M_{\tilde{q}} \approx 355 \mathrm{GeV} / \mathrm{c}^{2}\right)$ Same quantities as in the previous case are shown in Fig. 4.26. The drop at high gluino masses can be explained by the fact that it is a region where squark production dominates and topologies with only two jets in the final state are favoured. 

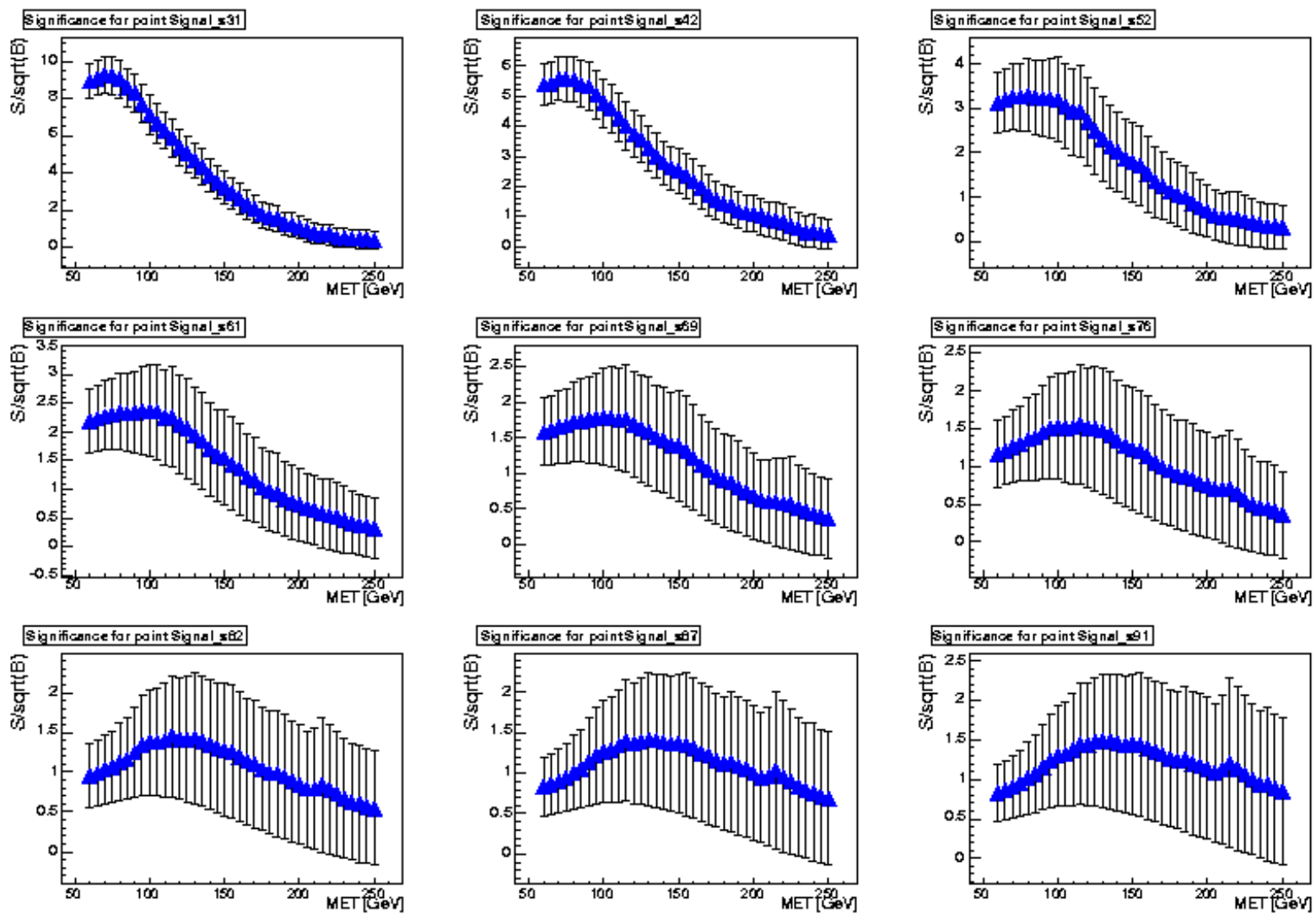

Fig. 4.17: $\mathrm{S} / \sqrt{\mathrm{B}}$ distributions for $\mathbb{H}_{T}$ of the mSUGRA points along the row. 

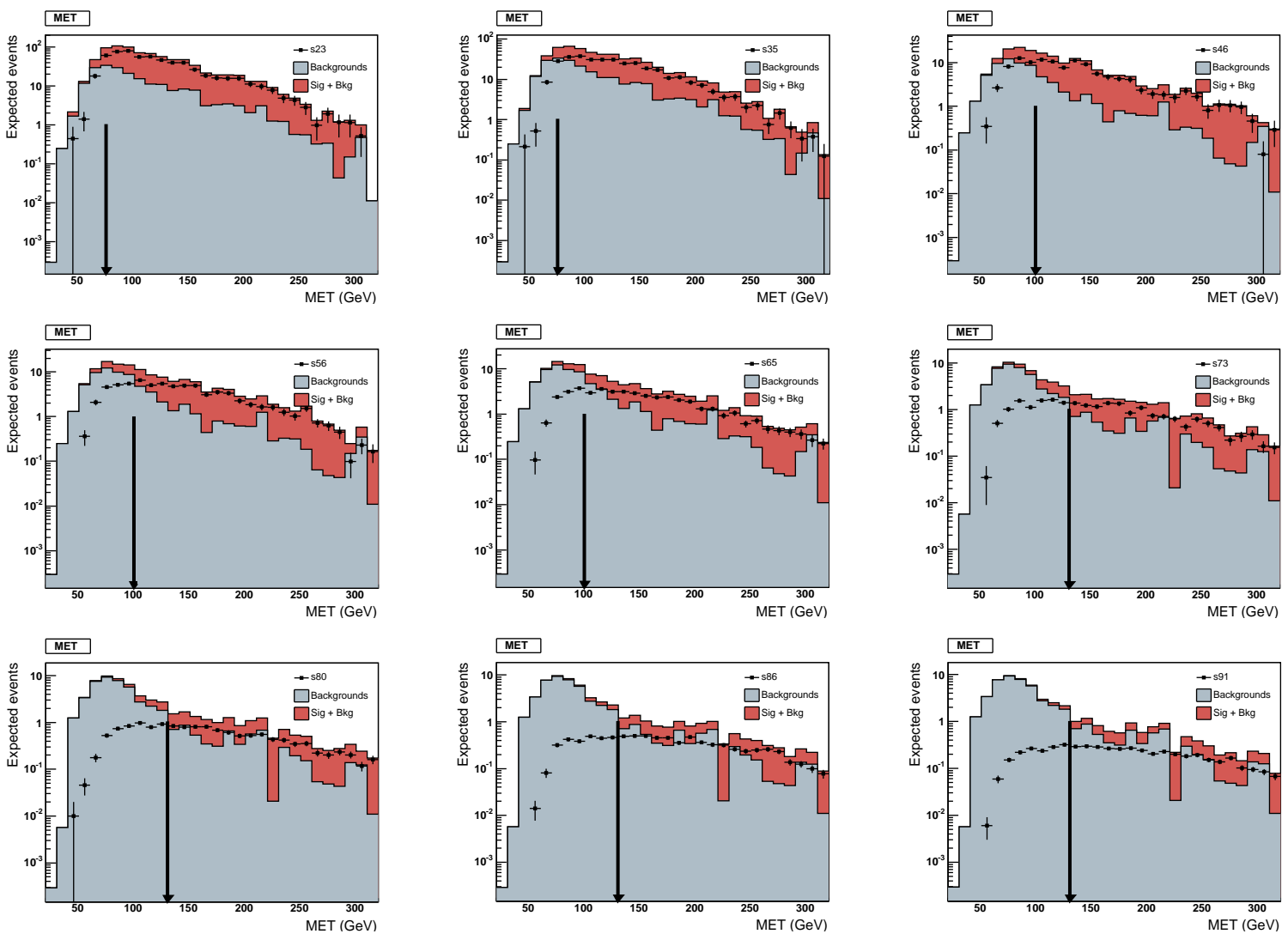

Fig. 4.18: $\not_{T}$ distributions for all the points in the diagonal $\left(\mathrm{M}_{\tilde{\mathrm{q}}} \approx \mathrm{M}_{\tilde{\mathrm{g}}}\right)$. The arrow shows where the cut is placed for each different zone. The first two histograms correspond to zone A (points s23, and s35), the next four plots correspond to zone B (points s46, s56, and s65), and the last three plots correspond to zone C (points s73, s80, s86, and s91). 

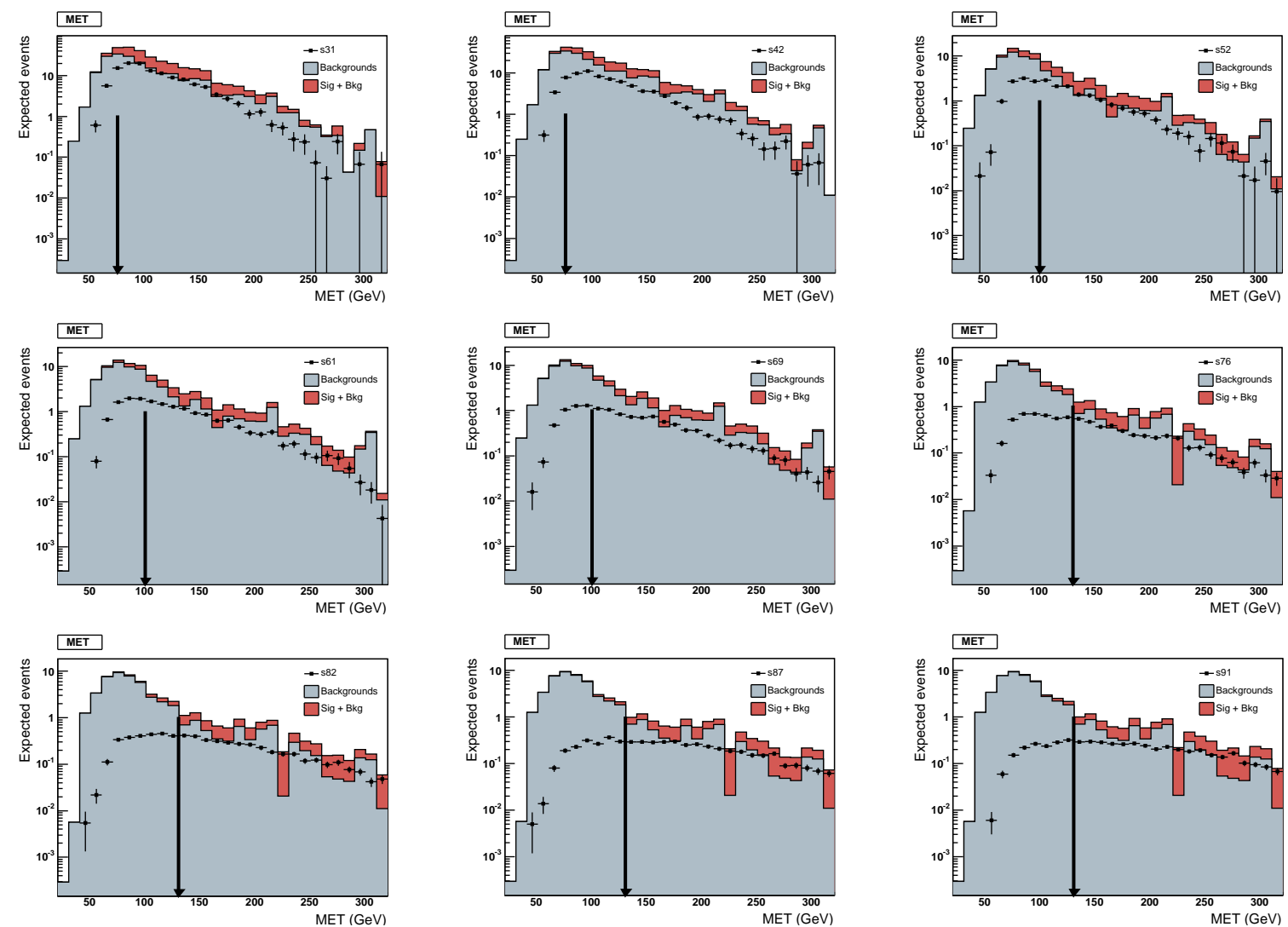

Fig. 4.19: $\not_{T}$ distributions for all the points in the chosen row (gluino mass increases as the point number increases). The arrow shows where the cut is placed for each different zone. The first two plots correspond to zone A (points s31, and s42), the next four plots correspond to zone B (points s52, s61, and s69), and the last three plots correspond to zone C (points s76, s82, s87, and s91). 

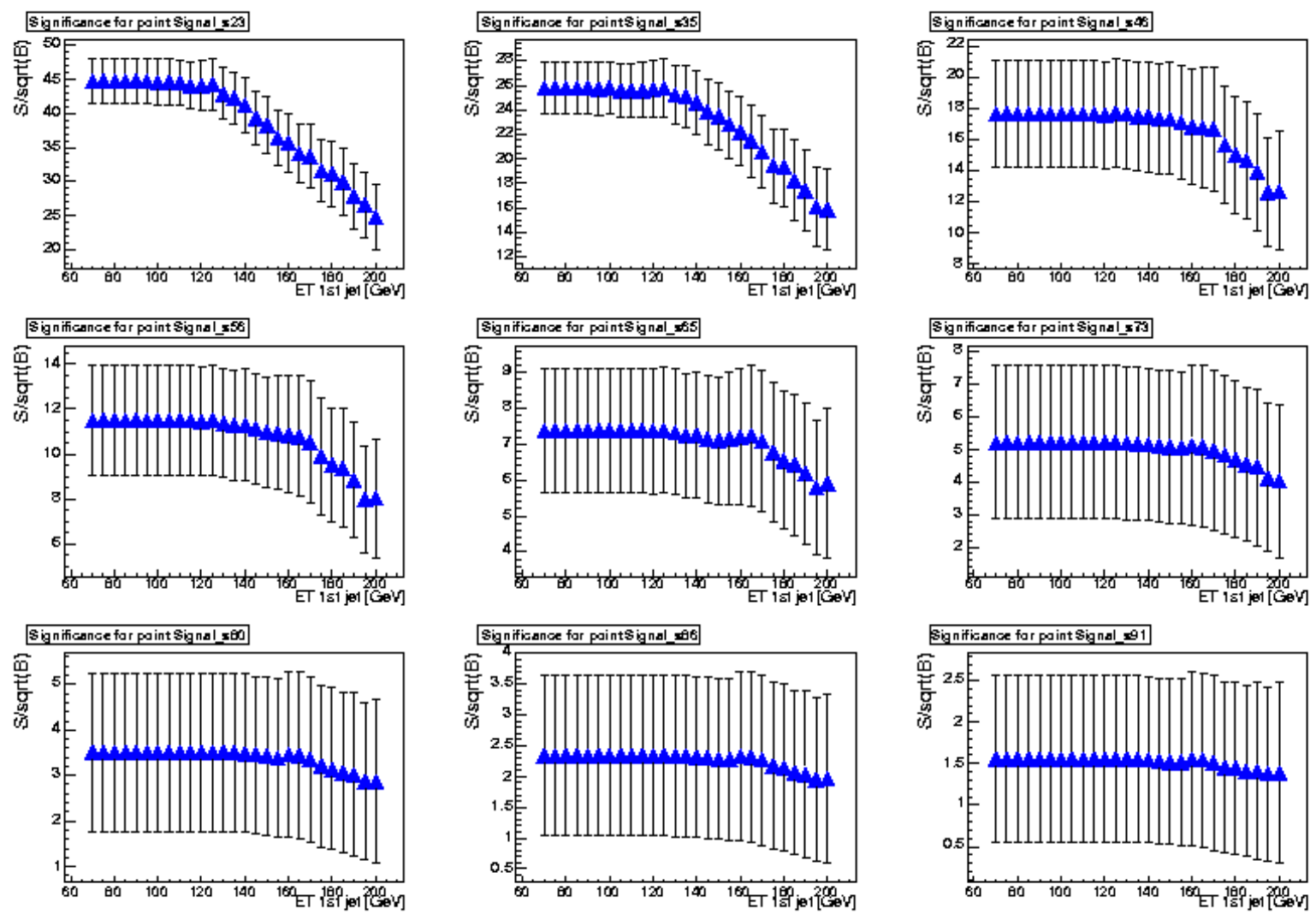

Fig. 4.20: $\mathrm{S} / \sqrt{\mathrm{B}}$ distribution for $\mathrm{E}_{\mathrm{T}}^{\text {jet } 1}$ of the mSUGRA points along the diagonal $\left(\mathrm{M}_{\tilde{\mathrm{q}}} \approx \mathrm{M}_{\tilde{\mathrm{g}}}\right)$. After applying the $\mathrm{H}_{\mathrm{T}}$ and $\mathscr{E}_{T}$, the signal and background distributions have the same shape. Therefore, the $\mathrm{S} / \sqrt{\mathrm{B}}$ becomes flat until it starts to drop. 

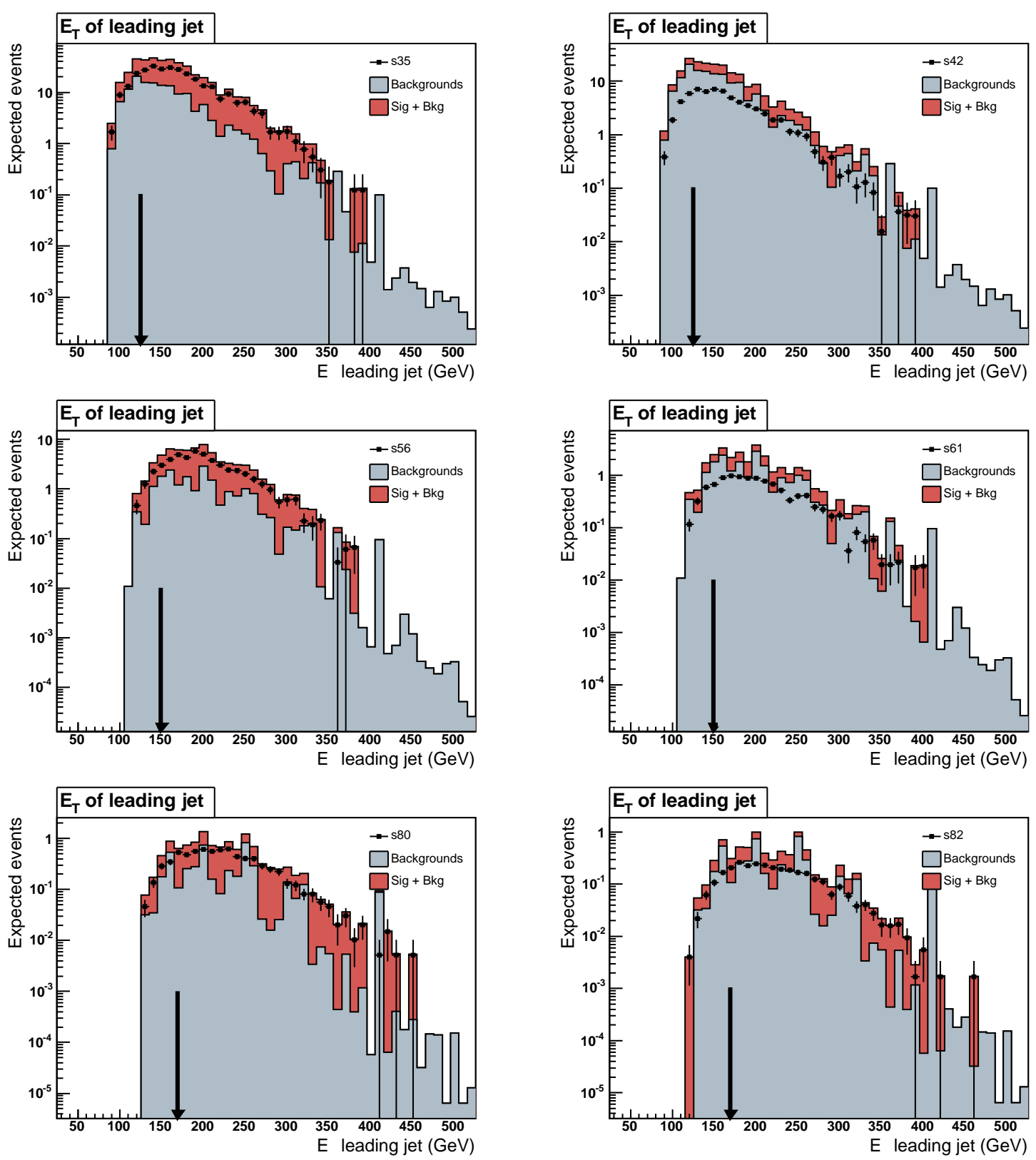

Fig. 4.21: $\mathrm{E}_{\mathrm{T}}^{\text {jet } 1}$ distributions for points $\mathrm{s} 35$, $\mathrm{s} 56$, and $\mathrm{s} 80$ on the left, and points $\mathrm{s} 42$, $\mathrm{s} 61$, and $\mathrm{s} 82$ on the right (each pair of histograms corresponds to points belonging to the same column (same gluino mass) in Fig. 4.3). The cut is chosen to remove the low-end tails of the signal distributions. 

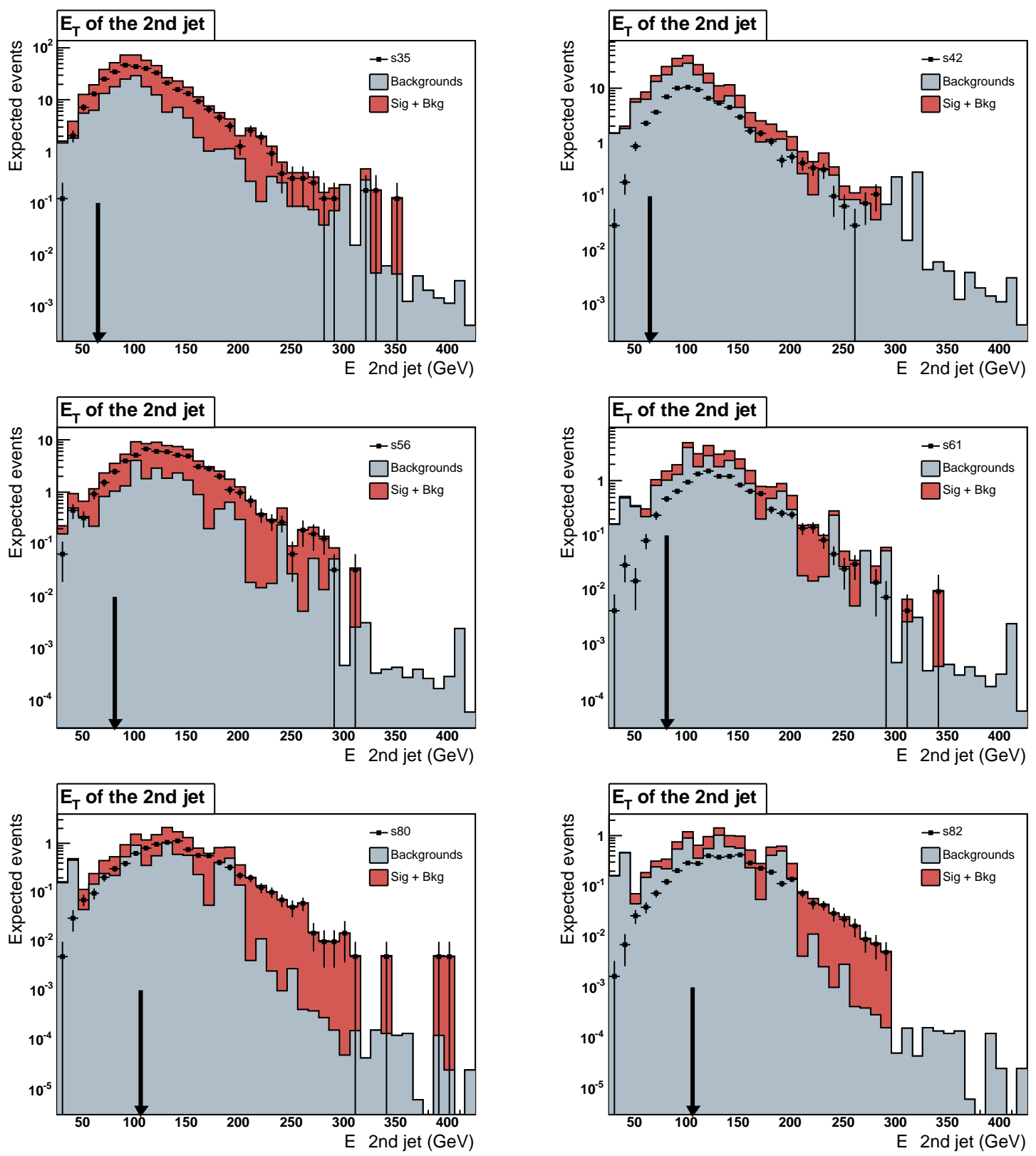

Fig. 4.22: $\mathrm{E}_{\mathrm{T}}^{\mathrm{jet} 2}$ distributions for points $\mathrm{s} 35$, $\mathrm{s} 56$, and $\mathrm{s} 80$ on the left, and points $\mathrm{s} 42$, $\mathrm{s} 61$, and $\mathrm{s} 82$ on the right (each pair of histograms corresponds to points belonging to the same column (same gluino mass) in Fig. 4.3). The cut is chosen to remove the low-end tails of the signal distributions. 

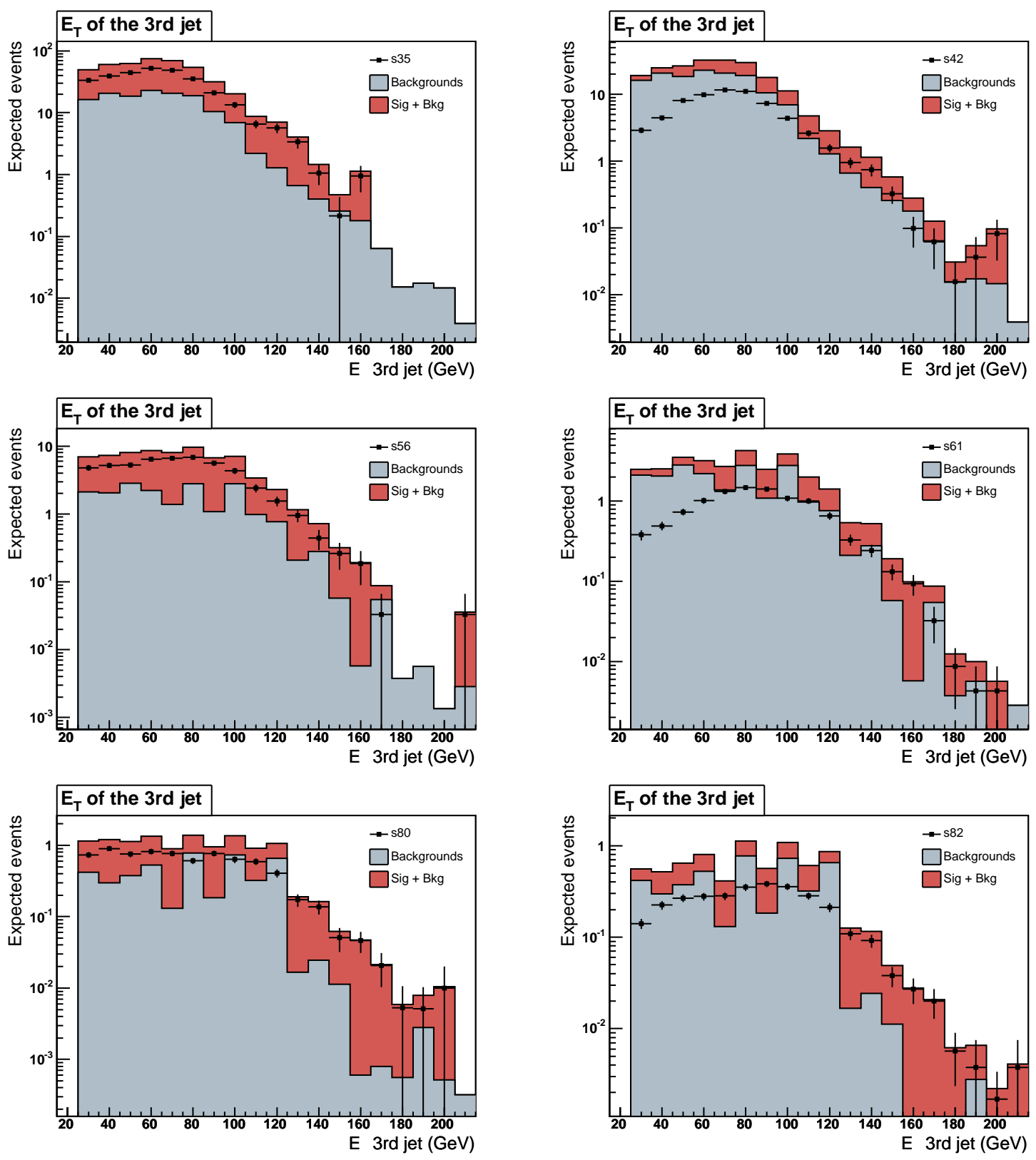

Fig. 4.23: $\mathrm{E}_{\mathrm{T}}^{\mathrm{jet} 3}$ distributions for points $\mathrm{s} 35$, $\mathrm{s} 56$, and $\mathrm{s} 80$ on the left, and points $\mathrm{s} 42$, s61, and $\mathrm{s} 82$ on the right (each pair of histograms corresponds to points belonging to the same column (same gluino mass) in Fig. 4.3). We do not apply a cut to the energy of the third jet except for the pre-selection cut of $25 \mathrm{GeV}$. 


\begin{tabular}{|c|c|c|c|}
\hline \hline Cuts & mSUGRA s35 & mSUGRA s56 & mSUGRA s80 \\
\hline Initial Exp. \# of Events & 2149 & 561 & 83 \\
\hline Vertex: $\left|V_{z}\right|<60 \mathrm{~cm}$ & $96.6 \pm 0.2 \%$ & $96.7 \pm 0.1 \%$ & $96.7 \pm 0.1 \%$ \\
\hline$\geq 3$ jets $\left(E_{T}>25 \mathrm{GeV}\right.$ and $\left.|\eta|<2.0\right)$ & $75.3 \pm 0.4 \%$ & $75.3 \pm 0.4 \%$ & $75.6 \pm 0.4 \%$ \\
\hline 1 Central jet $(\eta<1.1)$ & $75.2 \pm 0.4 \%$ & $74.9 \pm 0.4 \%$ & $75.3 \pm 0.4 \%$ \\
\hline $\mathscr{E}_{T}>70 \mathrm{GeV}$ & $47.0 \pm 0.4 \%$ & $55.0 \pm 0.4 \%$ & $61.7 \pm 0.4 \%$ \\
\hline EEMF $>0.15$ & $47.0 \pm 0.4 \%$ & $55.0 \pm 0.4 \%$ & $61.7 \pm 0.4 \%$ \\
\hline ECHF $>0.15$ & $46.6 \pm 0.4 \%$ & $54.7 \pm 0.4 \%$ & $61.1 \pm 0.4 \%$ \\
\hline \hline$\Delta \phi\left(\mathscr{E}_{T}\right.$, jets $)$ & $24.7 \pm 0.4 \%$ & $30.3 \pm 0.4 \%$ & $34.4 \pm 0.4 \%$ \\
\hline EMF of the jets & $23.2 \pm 0.3 \%$ & $28.3 \pm 0.4 \%$ & $32.0 \pm 0.4 \%$ \\
\hline Minv $<76$ or Minv $>106 \mathrm{GeV} / c^{2}$ & $23.2 \pm 0.3 \%$ & $28.3 \pm 0.4 \%$ & $32.0 \pm 0.4 \%$ \\
\hline$\Delta \phi\left(\mathbb{E}_{T}\right.$, max iso track) & $22.5 \pm 0.3 \%$ & $27.8 \pm 0.4 \%$ & $31.3 \pm 0.4 \%$ \\
\hline $\mathrm{E}_{\mathrm{T}}$ of the jets & $16.9 \pm 0.3 \%$ & $17.3 \pm 0.3 \%$ & $15.9 \pm 0.3 \%$ \\
\hline $\mathscr{E}_{T}$ & $16.0 \pm 0.3 \%$ & $14.7 \pm 0.3 \%$ & $11.3 \pm 0.3 \%$ \\
\hline $\mathrm{H}_{\mathrm{T}}$ & $14.5 \pm 0.2 \%$ & $12.8 \pm 0.2 \%$ & $10.6 \pm 0.2 \%$ \\
\hline \hline $\mathrm{N}_{\text {exp }}$ Events $\left(371 \mathrm{pb}^{-} 1\right)$ & $310.8 \pm 3.8$ & $71.6 \pm 0.9$ & $8.9 \pm 0.1$ \\
\hline \hline
\end{tabular}

Tab. 4.4: Efficiencies for mSUGRA events in regions A, B, and C. The points shown are representative for each region. 

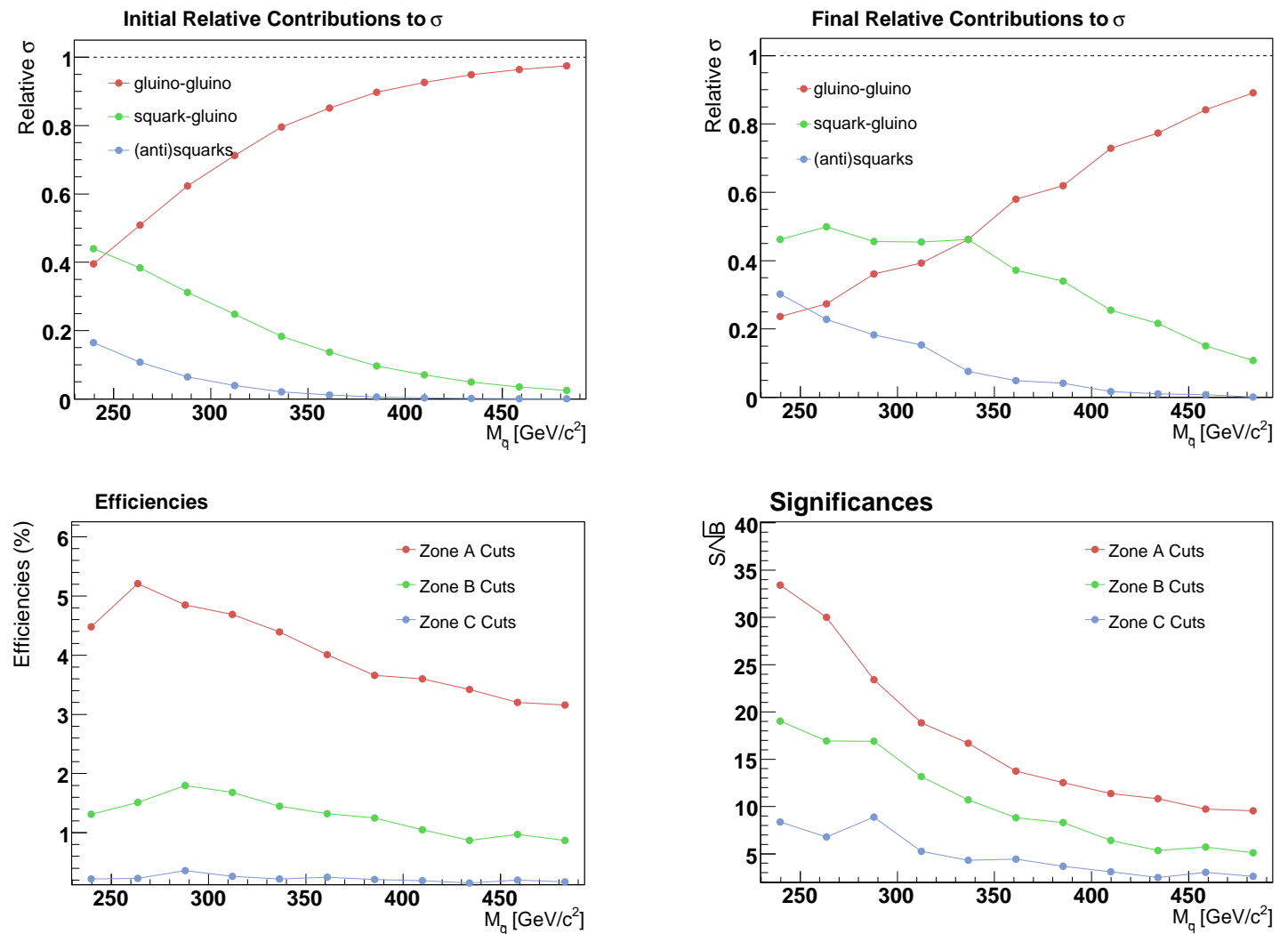

Fig. 4.24: Different relative contributions of the signal production processes before the cuts (top-left), after the analysis cuts (top-right), the signal efficiencies (bottom-left) and significances (bottom-right) along a column $\left(M_{\tilde{g}} \approx\right.$ $\left.185 \mathrm{GeV} / \mathrm{c}^{2}\right)$. 

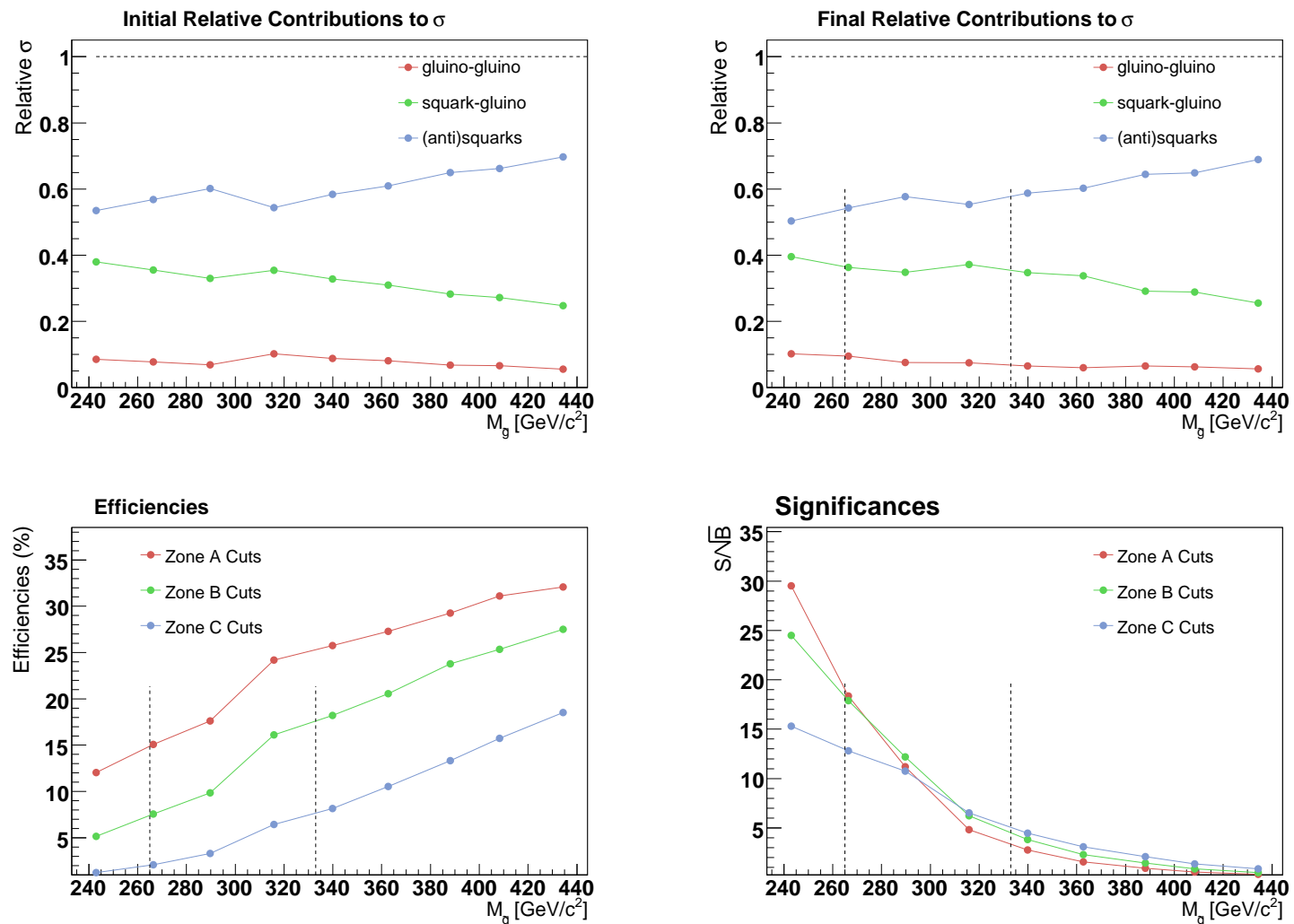

Fig. 4.25: Different relative contributions of the signal production processes before the cuts (top-left), after the analysis cuts (top-right), the signal efficiencies (bottom-left) and significances (bottom-right) along the diagonal ( $M_{\tilde{g}} \approx$ $\left.M_{\tilde{q}}\right)$. 

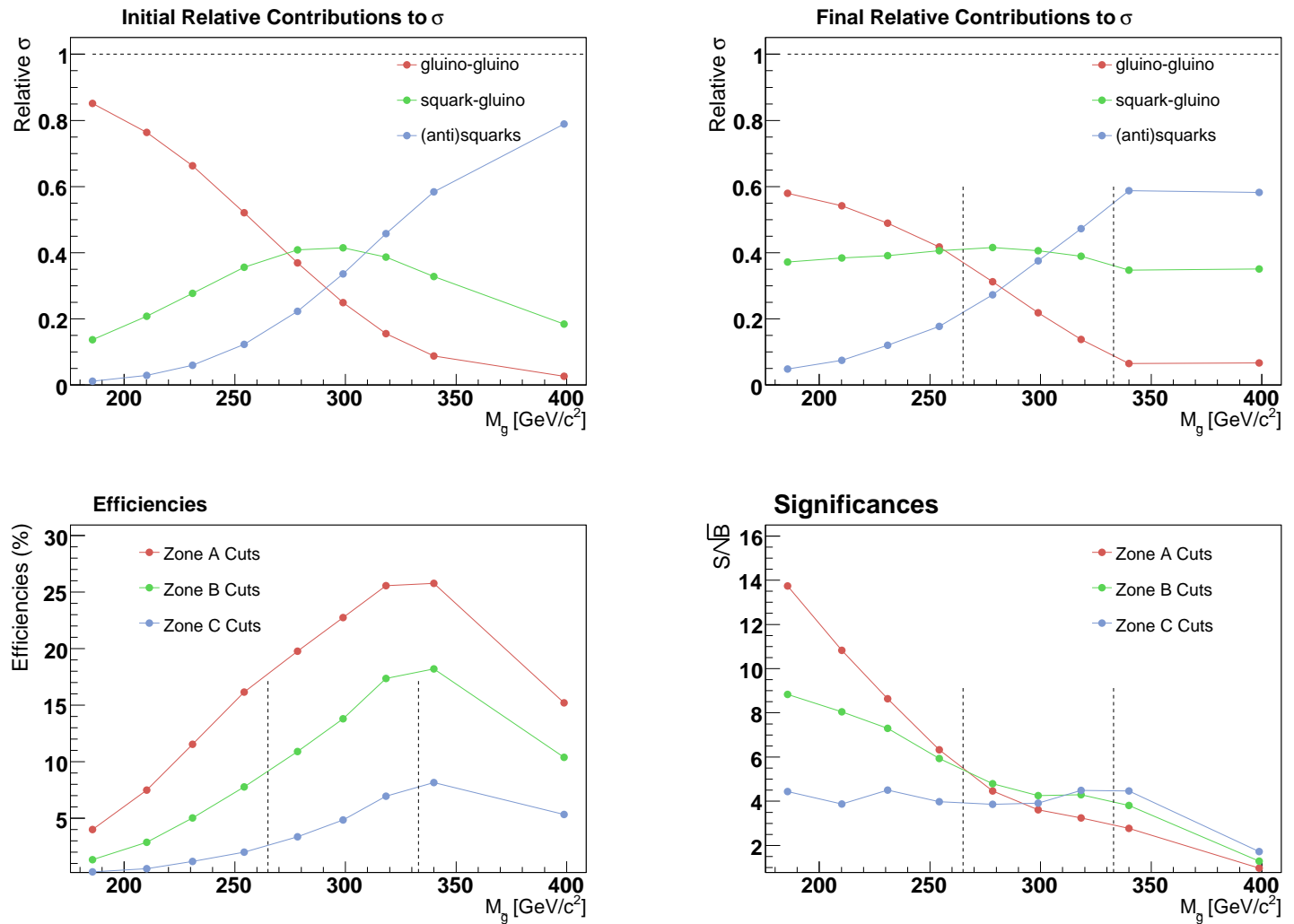

Fig. 4.26: Different relative contributions of the signal production processes before the cuts (top-left), after the analysis cuts (top-right), the signal efficiencies (bottom-left) and significances (bottom-right) along a row ( $M_{\tilde{q}} \approx$ $\left.355 \mathrm{GeV} / \mathrm{c}^{2}\right)$. 


\subsection{Systematic Uncertainties}

A complete study of systematic uncertainties on the estimation of signal and background expected events was carried out. The resulting systematic uncertainties for signal and background are collected in Tab. 4.5-4.8.

- Energy scale: Considered the uncertainties on the jet energy corrections and allowed $\pm 1 \sigma$ variation. The corresponding shift in the $\mathbb{E}_{T}$ was also included.

- Luminosity: The uncertainty on the total integrated luminosity at CDF is of 6\% [70].

- ISR/FSR: To estimate the systematic uncertainty on the expected signal events related to the modeling of the initial and final state radiation in the Monte Carlo, for each point in the mSUGRA mass plane additional samples were generated with modified $\Lambda_{\mathrm{QCD}}$ values.

In addition, the following two sources of theoretical uncertainty, related to renormalisation and factorisation scales, and PDFs, affect in particular the calculation of the NLO cross sections.

\subsubsection{Renormalisation Scale}

- PROSPINO Calculation: The default value of the renormalisation scale in PROSPINO is set as explained in Section 4.2. To investigate the effects of the renormalisation scale on the PROSPINO cross section the value of $\mu$ was shifted to $\mu * 2$ and $\mu / 2$. The systematic uncertainty associated with the renormalisation scale is then given by half the difference of the cross sections obtained from the previous two scenarios. The uncertainty due to the renormalisation scale on the signal cross section is shown in Fig. 4.42 and Fig. 4.43. The first plot shows the evolution of the cross section values along two columns (points with constant gluino mass). Likewise, the second plot shows the cross section when $\mathrm{M}_{\tilde{\mathrm{q}}} \approx \mathrm{M}_{\tilde{\mathrm{g}}}$ (diagonal). As it can be seen, this uncertainty is of the order of $20 \%$. Also shown in the plots is the combined uncertainty from the renormalisation scale and the PDF uncertainties (see below).

- MCFM Calculation: A similar strategy is used for the background processes whose NLO cross section is calculated using MCFM. However, for Z/W + jets only the uncertainty returned by setting the renormalisation to $2 * \mu$ is used. This is due to the fact that for $\mu / 2$ the renormalisation scale is in a region where the NLO cross section calculation is unstable [76].

- QCD Multijet Background: The uncertainty on the multijet background due to the renormalisation scale is $10 \%$, taken from [77]. 


\subsubsection{PDF uncertainty}

- Top Production: The top PDF uncertainty is taken directly from the theoretical calculation by Cacciari et al. [75]. For this analysis, a top mass of $175 \mathrm{GeV}$ is assumed.

- PROSPINO and MCFM Calculations: The Hessian method [78] was applied to calculate the uncertainty due to the choice of the PDF (CTEQ6L1 (LO) and CTEQ6.1M (NLO)). PROSPINO and MCFM were run using each of the 40 PDFs and the uncertainty associated was computed following the next recipe:

- For each eigenvalue, if the $+1 \sigma$ and $-1 \sigma$ PDFs produce a positive and a negative shift, respectively, then the positive and negative systematic uncertainties are given by the following formula: $\mathrm{d} X_{ \pm}^{2}=\sum_{i}^{20}\left(X_{ \pm}^{i}-X_{\mathrm{CTEQ} 6.1 \mathrm{M}}\right)^{2}$. Here, $X$, is the quantity for which one is considering the variations (e.g. the cross-section).

- If the $+1 \sigma$ and $-1 \sigma$ PDFs for a given eigenvalue result on just a positive shift with respect to the nominal value, only the largest uncertainty contributes to $\mathrm{d} X_{+}$. There is no contribution to $\mathrm{d} X_{-}$from this eigenvalue.

- If the $+1 \sigma$ and $-1 \sigma$ PDFs for a given eigenvalue result on just a negative shift with respect to the nominal value, only the largest uncertainty contributes to $\mathrm{d} X_{-}$. There is no contribution to $\mathrm{d} X_{+}$from this eigenvalue.

In the case of the signal, the uncertainty on the PDF produces dramatic consequences as shown in Fig. 4.27-4.29. The plots correspond to the cross section for each of the PROSPINO subprocesses for mSUGRA point 23,56 , and 80 . The largest source of uncertainty in the plots comes from eigenvalue 15 , which is associated with the high-x gluon content in the $\mathrm{PDF}^{4}$. The overall effect of the PDF uncertainty on the signal cross section is about $30 \%$ (Fig. 4.42 and Fig. 4.43). However, the uncertainty introduced by the PDFs on the signal cross section does not translate into a large effect on the signal efficiency (see Tab. 4.5). Fig. 4.30-4.32 show the distribution of the cross section for $W+2$ jets, $Z+2$ jets, and $W W$, respectively. Here, the uncertainty on the PDF has little effect on the calculation of the $k$-factor. It makes sense since the above background processes are dominated by contributions with $\mathrm{q} \overline{\mathrm{q}}$ in the initial state.

- QCD Multijet Background: A 20\% uncertainty on the QCD mutijet background is assumed based on the PDF studies on inclusive jet production at CDF [77].

In Fig. 4.33 the different contributions of the systematic uncertainties to the signal efficiency are shown for the three representative points close to the diagonal (mSUGRA s35, mSUGRA

\footnotetext{
${ }^{4}$ Note that each eigenvalue has an up and down contribution (PDF) associated. This is the reason why the eigenvalue 15 appears to be at 30 in the plot.
} 
s56 and mSUGRA s80). The most important contribution is the Jet Energy Scale, followed by ISR/FSR and the PDF uncertainties.

\begin{tabular}{|c|c|c|c|c|c|c|c|}
\hline \hline Region & Samples & JES & \llcorner & Renorm. & PDF & ISR/FSR & Total \\
\hline \multirow{2}{*}{ A } & mSUGRA s35 & $\pm 1.05 \%$ & $\pm 0.87 \%$ & $\pm 0.02 \%$ & $\pm 0.24 \%$ & $\pm 0.70 \%$ & $\pm \mathbf{1 . 5 5} \%$ \\
& (w.r.t nominal eff.) & $7.2 \%$ & $6.0 \%$ & $0.1 \%$ & $1.7 \%$ & $4.8 \%$ & $10.7 \%$ \\
\hline \multirow{2}{*}{ B } & mSUGRA s56 & $\pm 1.04 \%$ & $\pm 0.77 \%$ & $\pm 0.02 \%$ & $\pm 0.34 \%$ & $\pm 0.67 \%$ & $\pm \mathbf{1 . 5 0} \%$ \\
& (w.r.t nominal eff.) & $8.1 \%$ & $6.0 \%$ & $0.2 \%$ & $2.7 \%$ & $5.2 \%$ & $11.7 \%$ \\
\hline \multirow{2}{*}{$\mathrm{C}$} & mSUGRA s80 & $\pm 1.12 \%$ & $\pm 0.64 \%$ & $\pm 0.02 \%$ & $\pm 0.47 \%$ & $\pm 0.42 \%$ & $\pm \mathbf{1 . 4 3} \%$ \\
& (w.r.t nominal eff.) & $10.6 \%$ & $6.0 \%$ & $0.2 \%$ & $4.4 \%$ & $4.0 \%$ & $13.5 \%$ \\
\hline \hline
\end{tabular}

Tab. 4.5: Systematic uncertainties on the selection efficiency for some representative mSUGRA signal points. The upper numbers are the different uncertainties on the efficiencies and the lower numbers the uncertainties relative to the nominal efficiencies, quoted in Tab. 4.4.

\begin{tabular}{|c|c|c|c|c|c|}
\hline \hline Region & JES & $\iota$ & Renorm. & PDF & Total \\
\hline Region A & \pm 30.66 & \pm 12.66 & \pm 15.48 & \pm 23.78 & $\pm \mathbf{4 4 . 0 3}$ \\
(w.r.t. Exp. Bkg.) & $14 \%$ & $6 \%$ & $7 \%$ & $11 \%$ & $21 \%$ \\
\hline Region B & \pm 11.91 & \pm 3.34 & \pm 4.02 & \pm 5.66 & $\pm \mathbf{1 4 . 2 6}$ \\
(w.r.t. Exp. Bkg.) & $21 \%$ & $6 \%$ & $7 \%$ & $10 \%$ & $26 \%$ \\
\hline Region C & \pm 2.33 & \pm 0.49 & \pm 0.57 & \pm 0.85 & $\pm \mathbf{2 . 6 0}$ \\
(w.r.t. Exp. Bkg.) & $28 \%$ & $6 \%$ & $7 \%$ & $10 \%$ & $32 \%$ \\
\hline \hline
\end{tabular}

Tab. 4.6: Systematic uncertainties on the background expected number of events (in number of events and with respect to the total background expectations, quoted in Tab. 4.10) for the different regions considered in the analysis. 


\begin{tabular}{|c|c|c|c|}
\hline \hline Background & Region A & Region B & Region C \\
\hline QCD & \pm 7.11 & \pm 1.48 & \pm 0.22 \\
\hline$W W$ & \pm 0.07 & \pm 0.02 & \pm 0.00 \\
\hline$W \rightarrow e v$ & \pm 1.93 & \pm 0.53 & \pm 0.10 \\
\hline$W \rightarrow \mu \nu$ & \pm 0.07 & \pm 0.48 & \pm 0.12 \\
\hline$W \rightarrow \tau \nu$ & \pm 1.93 & \pm 0.60 & \pm 0.04 \\
\hline$Z / \gamma^{*} \rightarrow e e$ & \pm 0.07 & \pm 0.00 & \pm 0.00 \\
\hline$Z / \gamma^{*} \rightarrow \mu \mu$ & \pm 1.93 & \pm 0.02 & \pm 0.01 \\
\hline$Z / \gamma^{*} \rightarrow \tau \tau$ & \pm 0.07 & \pm 0.03 & \pm 0.00 \\
\hline$Z \rightarrow v \nu$ & \pm 1.93 & \pm 0.85 & \pm 0.07 \\
\hline \hline
\end{tabular}

Tab. 4.7: Systematic uncertainty on the background expected number of events due to the uncertainty on the renormalisation scale.

\begin{tabular}{|c|c|c|c|}
\hline \hline Background & Region A & Region B & Region C \\
\hline QCD & \pm 14.2 & \pm 2.97 & \pm 0.45 \\
\hline$t \bar{t}$ & \pm 5.78 & \pm 1.55 & \pm 0.25 \\
\hline$W W$ & \pm 0.07 & \pm 0.02 & \pm 0.00 \\
\hline$W \rightarrow e \nu$ & \pm 0.86 & \pm 0.24 & \pm 0.05 \\
\hline$W \rightarrow \mu \nu$ & \pm 0.75 & \pm 0.21 & \pm 0.05 \\
\hline$W \rightarrow \tau \nu$ & \pm 0.78 & \pm 0.27 & \pm 0.02 \\
\hline$Z / \gamma^{*} \rightarrow e e$ & \pm 0.00 & \pm 0.00 & \pm 0.00 \\
\hline$Z / \gamma^{*} \rightarrow \mu \mu$ & \pm 0.02 & \pm 0.01 & \pm 0.00 \\
\hline$Z / \gamma^{*} \rightarrow \tau \tau$ & \pm 0.04 & \pm 0.01 & \pm 0.00 \\
\hline$Z \rightarrow \nu \nu$ & \pm 1.23 & \pm 0.39 & \pm 0.03 \\
\hline \hline
\end{tabular}

Tab. 4.8: Systematic uncertainty on the background expected number of events due to the uncertainty on the PDFs. 

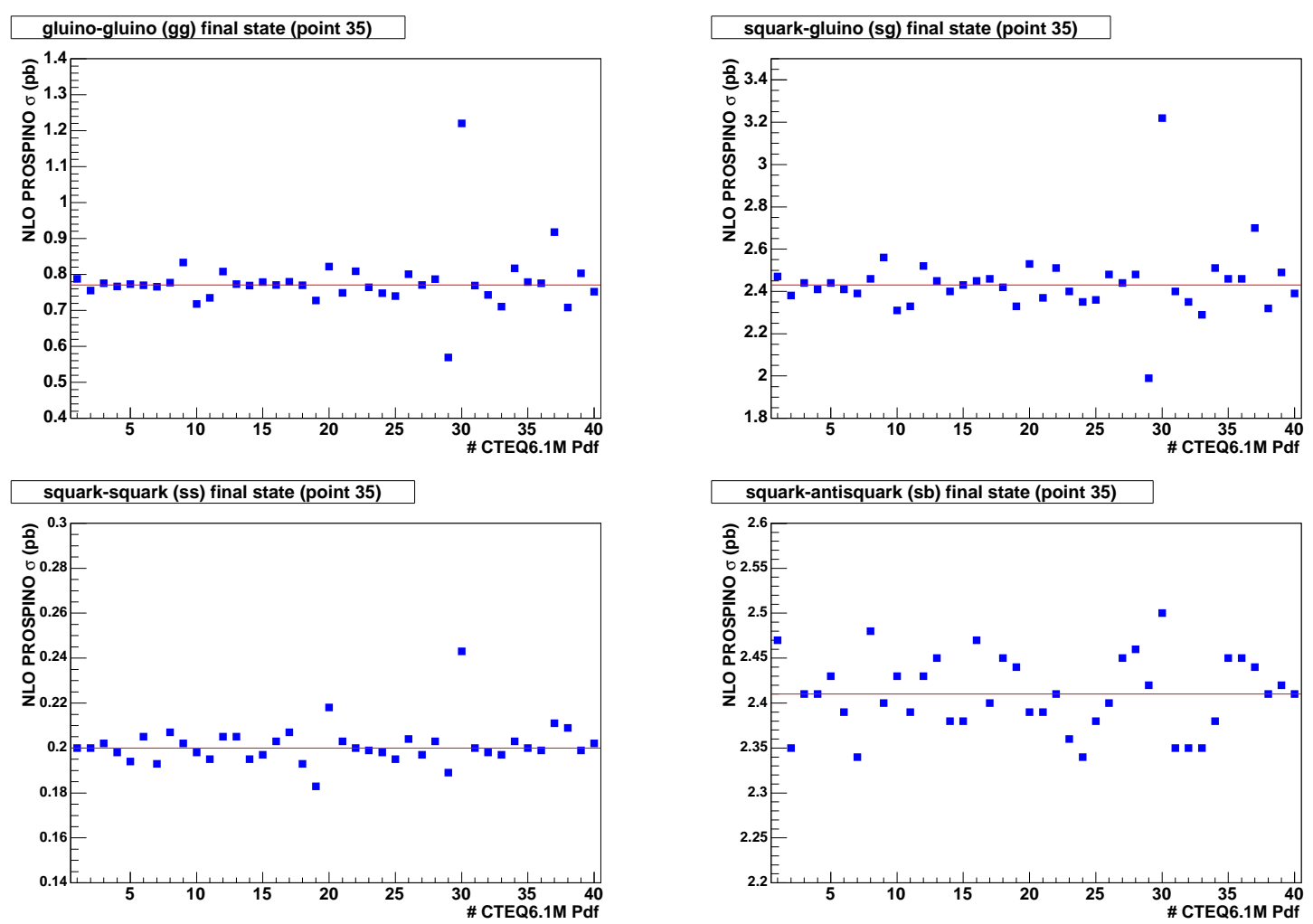

Fig. 4.27: $\sigma_{g g}, \sigma_{s g}, \sigma_{s s}$, and $\sigma_{s b}$ distributions for the 40 different PDFs (points) and the nominal PDF (line) for mSUGRA point 35 . Each eigenvalue has its plus and minus deviation represented in successive points. The largest deviation corresponds to eigenvalue 15 (in the points 29 and 30 of the plot) which is associated with the high-x gluon content. The $\sigma_{s b}$ plot does not show such deviation as it is dominated by $q \bar{q}$ annihilation. 

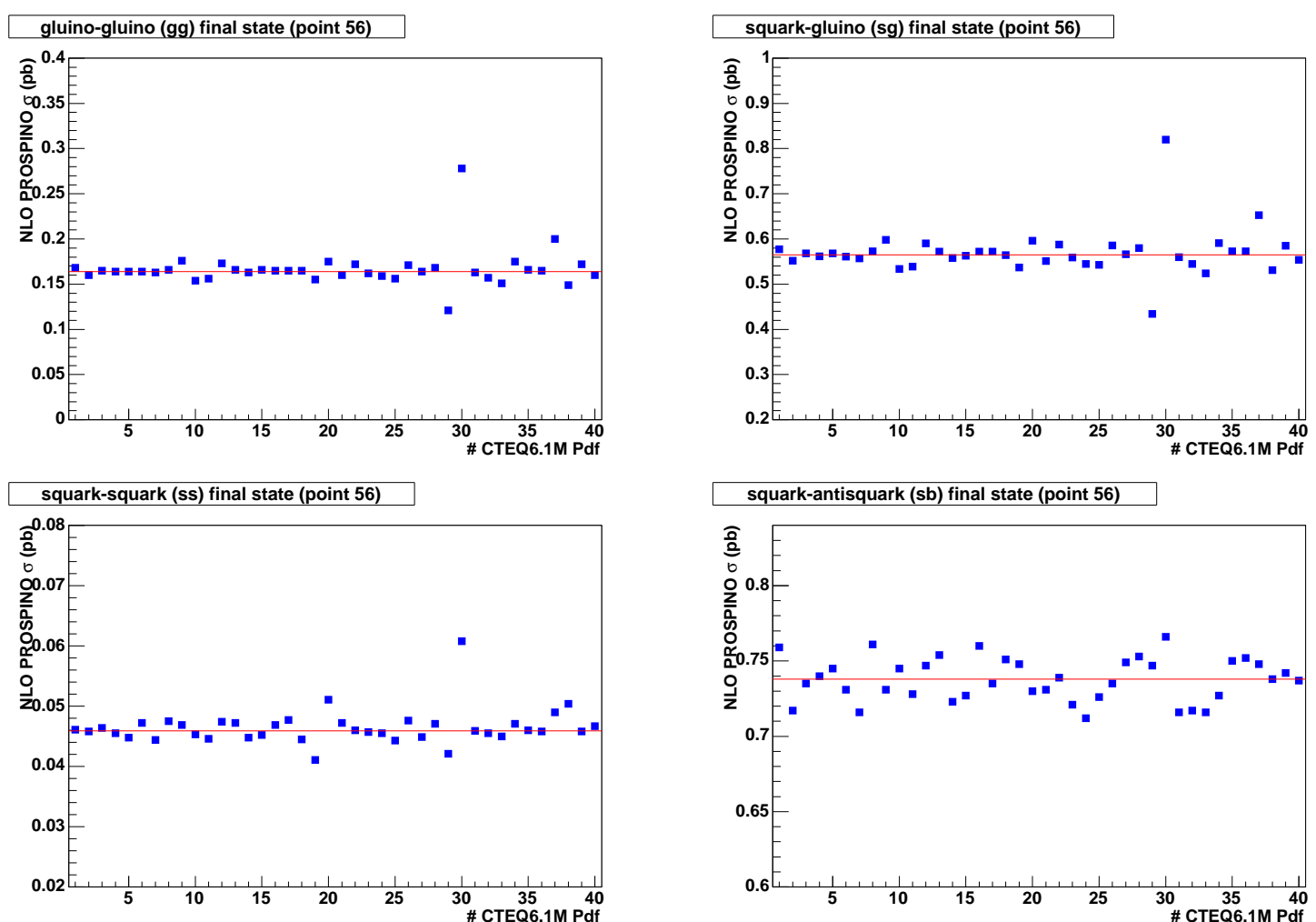

Fig. 4.28: $\sigma_{g g}, \sigma_{s g}, \sigma_{s s}$, and $\sigma_{s b}$ distributions for the 40 different PDFs (points) and the nominal PDF (line) for mSUGRA point 56. Each eigenvalue has its plus and minus deviation represented in successive points. The largest deviation corresponds to eigenvalue 15 (in the points 29 and 30 of the plot) which is associated with the high-x gluon content. The $\sigma_{s b}$ plot does not show such deviation as it is dominated by q⿳亠े annihilation. 

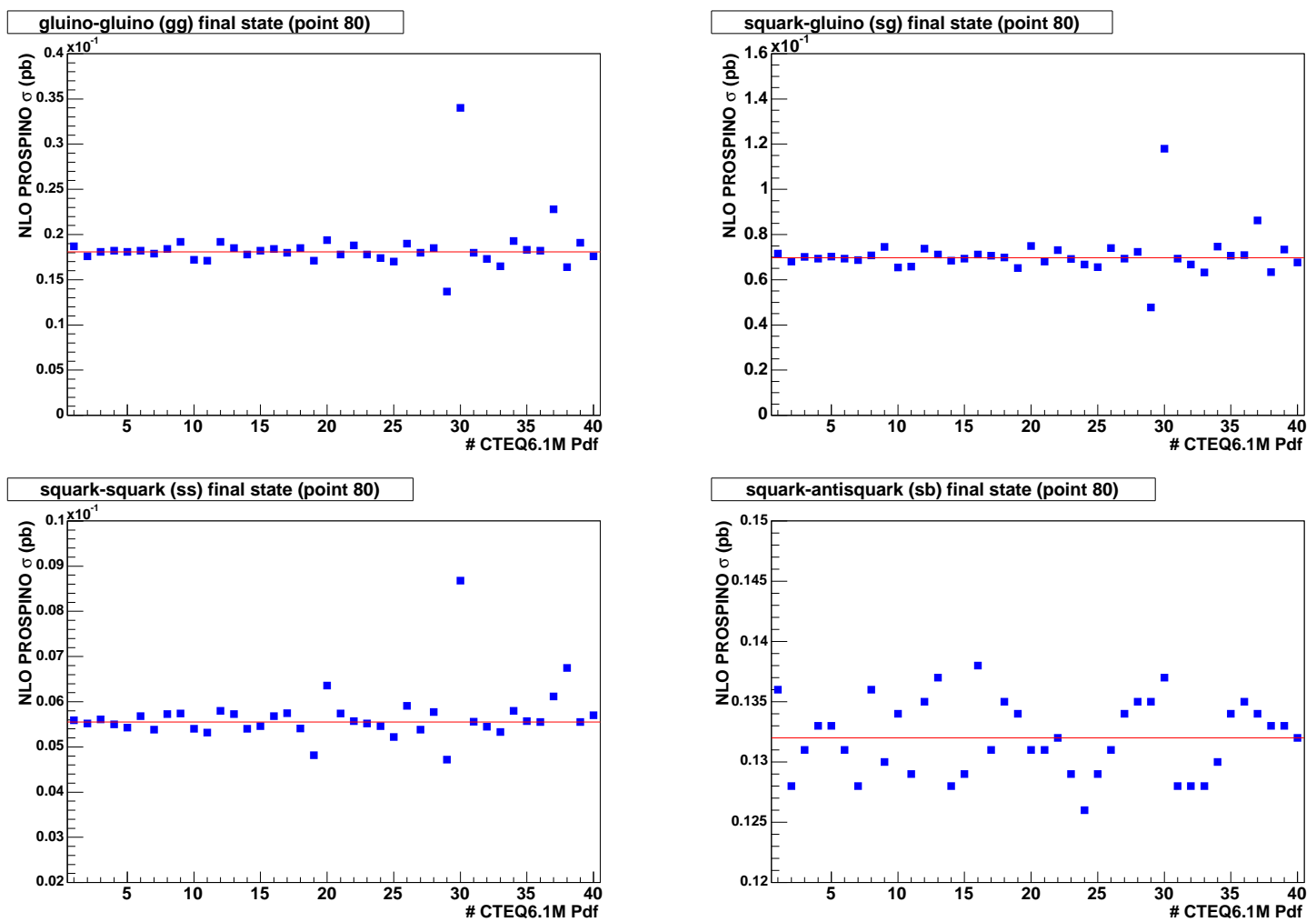

Fig. 4.29: $\sigma_{g g}, \sigma_{s g}, \sigma_{s s}$, and $\sigma_{s b}$ distributions for the 40 different PDFs (points) and the nominal PDF (line) for mSUGRA point 80 . Each eigenvalue has its plus and minus deviation represented in successive points. The largest deviation corresponds to eigenvalue 15 (in the points 29 and 30 of the plot) which is associated with the high-x gluon content. The $\sigma_{s b}$ plot does not show such deviation as it is dominated by $\mathrm{q} \overline{\mathrm{q}}$ annihilation.

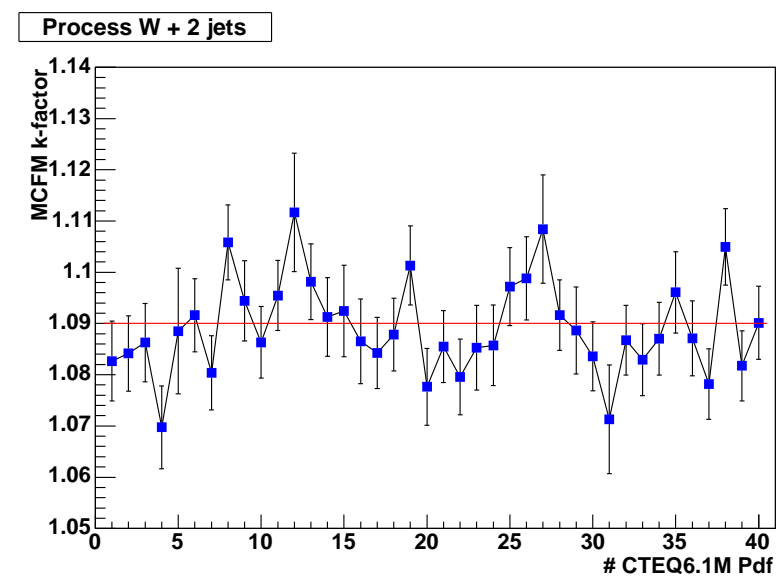

Fig. 4.30: $k$-factors for the 40 different PDFs (points) and the nominal PDF (line) for $W+2$ jets 


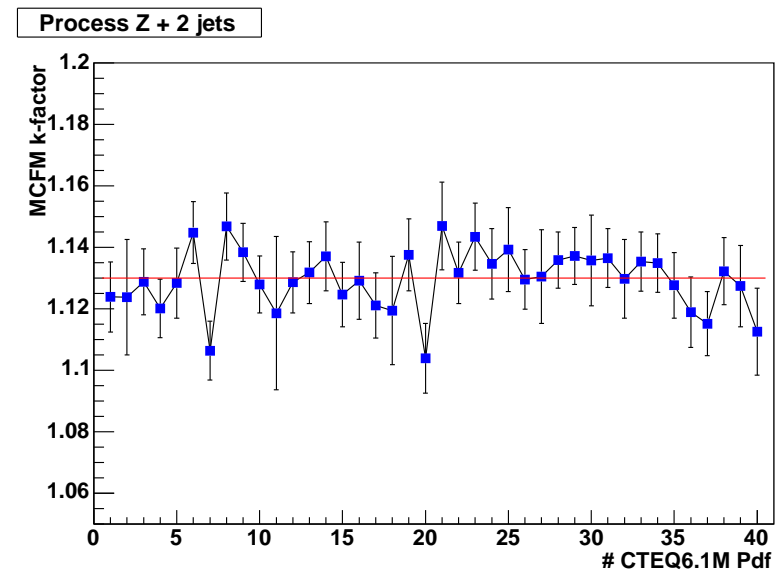

Fig. 4.31: $k$-factors for the 40 different PDFs (points) and the nominal PDF (line) for $Z+2$ jets.

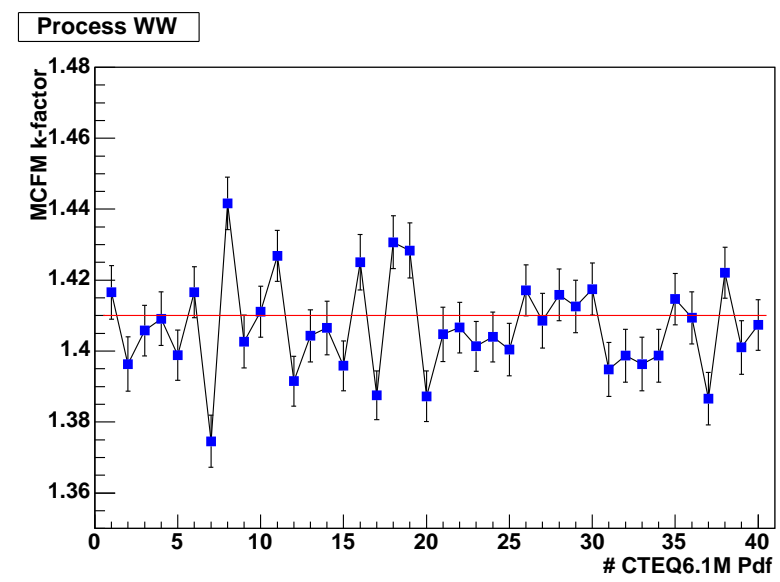

Fig. 4.32: $k$-factors for the 40 different PDFs (points) and the nominal PDF (line) for $W W$ production. 


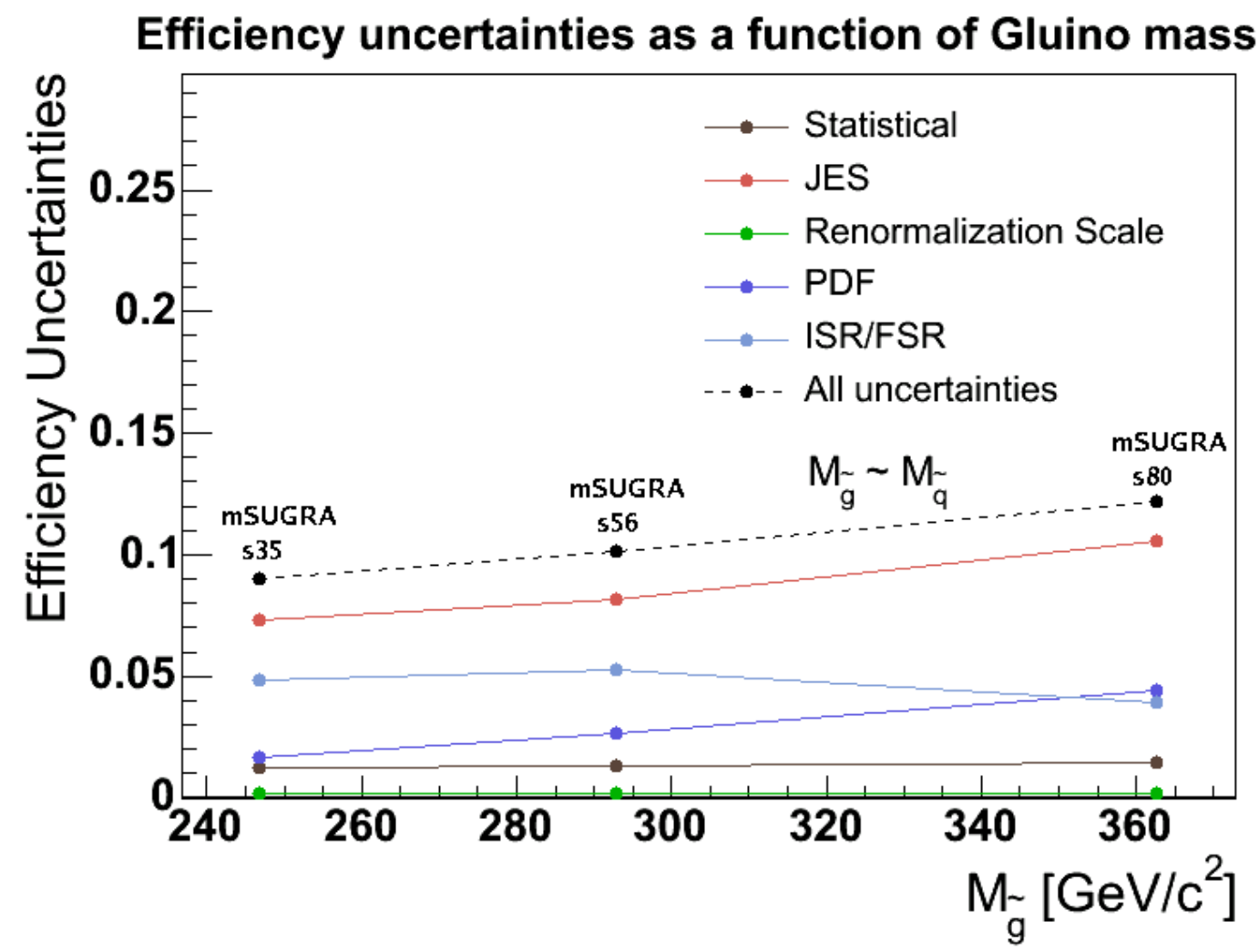

Fig. 4.33: Systematic uncertainties relative to the signal efficiencies for the three representative mSUGRA points s35, s56 and s80. 


\subsection{Results}

In this section the number of observed events, and expected background are presented for a total luminosity of $371 \mathrm{pb}^{-1}$. Tab. 4.9 gives the number of data events observed in each region defined in the analysis. Tab. 4.10 shows the total number of background expected events for each of the regions. A breakdown of the different backgrounds in each of the regions is included in Tab. 4.11-Tab. 4.13. Fig. 4.34 shows the different relative contributions to the overall background after all the cuts have been applied and Fig. 4.35-4.40 show the $\mathrm{H}_{\mathrm{T}}$ and $\mathbb{E}_{T}$ distributions for the final results. In these plots, all cuts have been applied except the one on the variable that is represented. The arrows in the figures indicate the position where the cuts on this variable is placed. Each figure shows the data together with the Monte Carlo predictions for the SM background and a representative mSUGRA signal mass point from the correspondent optimised region. The background Monte Carlo predictions provide a reasonable description of the data in all regions and no excess with respect to the SM predictions is observed. In addition, the $\Delta \phi\left(j e t, \mathbb{E}_{\mathrm{T}}\right)$ and the EMF requirements were reversed to enhance the QCD and boson+jets electromagnetic backgrounds separately, and test that the Monte Carlo properly describes each different background contribution. The muon contribution has also been studied reversing the muon cuts (asking for at least one isolated track). All these distributions are shown in Appendix G.

\begin{tabular}{|c|c|c|c|}
\hline Cuts & Bkg. Region A & Bkg. Region B & Bkg. Region C \\
\hline GoodRun List & \multicolumn{3}{|c|}{$9.93 \times 10^{6}$} \\
\hline Trigger (MET35 + 2 jets) & \multicolumn{3}{|c|}{$3.10 \times 10^{6}$} \\
\hline Vertex: $\left|V_{z}\right|<60 \mathrm{~cm}$ & \multicolumn{3}{|c|}{$2.32 \times 10^{6}$} \\
\hline$\geq 3$ jets $\left(E_{T}>25 \mathrm{GeV}\right.$ and $\left.\eta<2.0\right)$ & \multicolumn{3}{|c|}{356713} \\
\hline 1 Central jet $(\eta<1.1)$ & \multicolumn{3}{|c|}{345771} \\
\hline $\mathbb{E}_{T}>70 \mathrm{GeV}$ & \multicolumn{3}{|c|}{15414} \\
\hline EEMF $>0.15$ & \multicolumn{3}{|c|}{14090} \\
\hline $\mathrm{ECHF}>0.15$ & \multicolumn{3}{|c|}{13127} \\
\hline$\Delta \phi\left(\mathscr{E}_{T}, \mathrm{jets}\right)$ & \multicolumn{3}{|c|}{2301} \\
\hline EMF of the jets & \multicolumn{3}{|c|}{1425} \\
\hline Minv $<76$ or Minv $>106 \mathrm{GeV} / c^{2}$ & \multicolumn{3}{|c|}{1410} \\
\hline$\Delta \phi\left(\mathbb{E}_{T}, \max\right.$ iso track $)$ & \multicolumn{3}{|c|}{1204} \\
\hline $\mathrm{E}_{\mathrm{T}}$ of the jets & 296 & 141 & 39 \\
\hline $\mathbb{E}_{T}$ & 250 & 62 & 4 \\
\hline $\mathrm{H}_{\mathrm{T}}$ & $185 \pm 14$ & $40 \pm 6$ & $2_{-1.3}^{+2.6}$ \\
\hline
\end{tabular}

Tab. 4.9: Observed number of data events for regions A, B, and C. The optimisation procedure for the final three cuts is explained in the previous section. These numbers are to be compared with the ones given in Tab. 4.10. 


\begin{tabular}{|c|c|c|c|}
\hline Cuts & Bkg. Region A & Bkg. Region B & Bkg. Region C \\
\hline Initial Exp. \# of Events & \multicolumn{3}{|c|}{$9.83 \times 10^{6}$} \\
\hline Vertex: $\left|V_{z}\right|<60 \mathrm{~cm}$ & \multicolumn{3}{|c|}{$(2.878 \pm 0.001) \times 10^{6}$} \\
\hline$\geq 3$ jets $\left(E_{T}>25 \mathrm{GeV}\right.$ and $\left.\eta<2.0\right)$ & \multicolumn{3}{|c|}{$(8.898 \pm 0.007) \times 10^{5}$} \\
\hline 1 Central jet $(\eta<1.1)$ & \multicolumn{3}{|c|}{$(8.707 \pm 0.007) \times 10^{5}$} \\
\hline$\#_{T}>70 \mathrm{GeV}$ & \multicolumn{3}{|c|}{$12645 \pm 61$} \\
\hline $\mathrm{EEMF}>0.15$ & \multicolumn{3}{|c|}{$12642 \pm 61$} \\
\hline $\mathrm{ECHF}>0.15$ & \multicolumn{3}{|c|}{$12518 \pm 60$} \\
\hline$\Delta \phi\left(\not_{T}, j e t s\right)$ & \multicolumn{3}{|c|}{$2527.8 \pm 22.6$} \\
\hline EMF of the jets & \multicolumn{3}{|c|}{$1606.4 \pm 19.1$} \\
\hline Minv $<76$ or Minv $>106 \mathrm{GeV} / c^{2}$ & \multicolumn{3}{|c|}{$1587.5 \pm 19.1$} \\
\hline$\Delta \phi\left(\mathbb{E}_{T}, \max\right.$ iso track $)$ & \multicolumn{3}{|c|}{$1291.2 \pm 18.0$} \\
\hline $\mathrm{E}_{\mathrm{T}}$ of the jets & $325.3 \pm 8.6$ & $145.0 \pm 5.6$ & $58.1 \pm 3.5$ \\
\hline$\not_{T}$ & $276.8 \pm 7.9$ & $76.3 \pm 3.9$ & $11.0 \pm 1.4$ \\
\hline $\mathrm{H}_{\mathrm{T}}$ & $211.0 \pm 6.9$ & $55.7 \pm 3.4$ & $8.2 \pm 1.2$ \\
\hline Total Expected with Systematics & $211.0 \pm 6.9 \pm 44.0$ & $55.7 \pm \mathbf{3 . 4} \pm \mathbf{1 4 . 3}$ & $8.2 \pm 1.2 \pm \mathbf{2 . 6}$ \\
\hline
\end{tabular}

Tab. 4.10: Expected number of background events for regions A, B, and C. Recall that the last three cuts are optimised differently for each of the regions. 


\begin{tabular}{|c|c|c|c|}
\hline Cuts & $W \rightarrow e v+3$ jets & $W \rightarrow \mu \nu+3$ jets & $W \rightarrow \tau \nu+2$ jets \\
\hline Initial Exp. \# of Events & 36214 & 36214 & 99710 \\
\hline Vertex: $\left|V_{z}\right|<60 \mathrm{~cm}$ & $34910 \pm 13.1$ & $34898 \pm 12.6$ & $96154 \pm 40.9$ \\
\hline$\geq 3$ jets $\left(E_{T}>25 \mathrm{GeV}\right.$ and $\left.\eta<2.0\right)$ & $6974 \pm 27.7$ & $1840 \pm 14.7$ & $4783 \pm 47.1$ \\
\hline 1 Central jet $(\eta<1.1)$ & $6758 \pm 27.4$ & $1784 \pm 14.5$ & $4632 \pm 46.4$ \\
\hline $\mathbb{E}_{T}>70 \mathrm{GeV}$ & $1041 \pm 11.7$ & $505 \pm 7.9$ & $585 \pm 16.8$ \\
\hline EEMF $>0.15$ & $1041 \pm 11.7$ & $505 \pm 7.9$ & $585 \pm 16.8$ \\
\hline $\mathrm{ECHF}>0.15$ & $1027 \pm 11.7$ & $501 \pm 7.8$ & $575 \pm 16.7$ \\
\hline$\Delta \phi\left(\mathbb{E}_{T}\right.$, jets $)$ & $820 \pm 10.5$ & $387 \pm 6.9$ & $375 \pm 13.5$ \\
\hline EMF of the jets & $191 \pm 5.1$ & $346 \pm 6.5$ & $295 \pm 12.0$ \\
\hline Minv $<76$ or Minv $>106 \mathrm{GeV} / c^{2}$ & $191 \pm 5.1$ & $345 \pm 6.5$ & $295 \pm 12.0$ \\
\hline$\Delta \phi\left(\mathbb{E}_{T}, \max\right.$ iso track $)$ & $182 \pm 5.0$ & $190 \pm 4.8$ & $272 \pm 11.5$ \\
\hline \multicolumn{4}{|c|}{ Region A } \\
\hline $\mathrm{E}_{\mathrm{T}}^{\mathrm{jets}}>95,55$, and $25 \mathrm{GeV}$ & $36.2 \pm 2.2$ & $33.2 \pm 2.0$ & $36.6 \pm 4.2$ \\
\hline$E_{T}>75 \mathrm{GeV}$ & $31.5 \pm 2.1$ & $29.8 \pm 1.9$ & $31.2 \pm 3.9$ \\
\hline $\mathrm{H}_{\mathrm{T}}>230 \mathrm{GeV}$ & $23.3 \pm 1.8$ & $20.5 \pm 1.6$ & $21.5 \pm 3.2$ \\
\hline \multicolumn{4}{|c|}{ Region B } \\
\hline $\mathrm{E}_{\mathrm{T}}^{\mathrm{jets}}>120,70$, and $25 \mathrm{GeV}$ & $17.3 \pm 1.5$ & $13.4 \pm 1.3$ & $14.1 \pm 2.6$ \\
\hline $\mathbb{E}_{T}>90 \mathrm{GeV}$ & $8.9 \pm 1.1$ & $9.0 \pm 1.1$ & $7.8 \pm 2.0$ \\
\hline $\mathrm{H}_{\mathrm{T}}>280 \mathrm{GeV}$ & $6.4 \pm 0.9$ & $5.8 \pm 0.9$ & $7.3 \pm 1.9$ \\
\hline \multicolumn{4}{|c|}{ Region C } \\
\hline $\mathrm{E}_{\mathrm{T}}^{\mathrm{jets}}>140,100$, and $25 \mathrm{GeV}$ & $8.3 \pm 1.1$ & $4.5 \pm 0.7$ & $4.9 \pm 1.5$ \\
\hline $\mathbb{E}_{T}>120 \mathrm{GeV}$ & $1.5 \pm 0.5$ & $2.2 \pm 0.5$ & $1.0 \pm 0.7$ \\
\hline $\mathrm{H}_{\mathrm{T}}>330 \mathrm{GeV}$ & $1.2 \pm 0.4$ & $1.5 \pm 0.4$ & $0.5 \pm 0.5$ \\
\hline
\end{tabular}

Tab. 4.11: Expected number of background events from Standard Model W production processes. Only statistical uncertainties are shown. 


\begin{tabular}{|c|c|c|c|c|}
\hline Cuts & $Z \rightarrow \mathrm{vv}+3$ jets & $Z / \gamma^{*} \rightarrow e e+2$ jets & $Z / \gamma^{*} \rightarrow \mu \mu+2$ jets & $Z / \gamma^{*} \rightarrow \tau \tau+2$ jets \\
\hline Initial Exp. \# of Events & 21800 & 32441 & 32441 & 32441 \\
\hline Vertex: $\left|V_{z}\right|<60 \mathrm{~cm}$ & $21080 \pm 12.0$ & $31276 \pm 8.4$ & $31282 \pm 8.7$ & $31287 \pm 8.3$ \\
\hline 3 jets & $1039 \pm 14.9$ & $4243 \pm 11.5$ & $424 \pm 5.1$ & $1887 \pm 8.8$ \\
\hline 1 Central jet $(\eta<1.1)$ & $1004 \pm 14.6$ & $4081 \pm 11.3$ & $408 \pm 5.0$ & $1816 \pm 8.6$ \\
\hline$E_{T}>70 \mathrm{GeV}$ & $320 \pm 8.4$ & $31 \pm 0.9$ & $65 \pm 1.7$ & $67 \pm 1.6$ \\
\hline EEMF $>0.15$ & $320 \pm 8.4$ & $31 \pm 0.9$ & $65 \pm 1.7$ & $67 \pm 1.6$ \\
\hline ECHF $>0.15$ & $317 \pm 8.3$ & $30 \pm 0.9$ & $64 \pm 1.7$ & $66 \pm 1.6$ \\
\hline$\Delta \phi\left(E_{T}\right.$, jets $)$ & $240 \pm 7.3$ & $7.4 \pm 0.4$ & $48 \pm 1.5$ & $29 \pm 1.0$ \\
\hline EMF of the jets & $214 \pm 6.9$ & $1.1 \pm 0.2$ & $43 \pm 1.4$ & $22 \pm 0.9$ \\
\hline $\mathrm{M}_{\text {inv }}$ window & $214 \pm 6.9$ & $0.9 \pm 0.2$ & $27 \pm 1.2$ & $22 \pm 0.9$ \\
\hline$\Delta \phi\left(E_{T}, \max\right.$ iso track $)$ & $214 \pm 6.9$ & $0.2 \pm 0.1$ & $9.2 \pm 0.7$ & $11 \pm 0.7$ \\
\hline \multicolumn{5}{|c|}{ Region A } \\
\hline $\mathrm{E}_{\mathrm{T}}^{\mathrm{jets}}>95,55$, and $25 \mathrm{GeV}$ & $43.5 \pm 3.11$ & $0.03 \pm 0.10$ & $1.5 \pm 0.30$ & $2.4 \pm 0.31$ \\
\hline$\not_{T}>75 \mathrm{GeV}$ & $38.8 \pm 2.94$ & $0.02 \pm 0.10$ & $1.3 \pm 0.28$ & $1.8 \pm 0.26$ \\
\hline $\mathrm{H}_{\mathrm{T}}>230 \mathrm{GeV}$ & $27.9 \pm 2.49$ & $0.02 \pm 0.10$ & $0.51 \pm 0.15$ & $1.4 \pm 0.23$ \\
\hline \multicolumn{5}{|c|}{ Region B } \\
\hline $\mathrm{E}_{\mathrm{T}}^{\mathrm{jets}}>120,70$, and $25 \mathrm{GeV}$ & $19.4 \pm 2.08$ & $0.00 \pm 0.10$ & $0.36 \pm 0.14$ & $1.1 \pm 0.21$ \\
\hline$\not_{T}>90 \mathrm{GeV}$ & $14.1 \pm 1.77$ & $0.00 \pm 0.10$ & $0.22 \pm 0.13$ & $0.47 \pm 0.13$ \\
\hline $\mathrm{H}_{\mathrm{T}}>280 \mathrm{GeV}$ & $8.7 \pm 1.39$ & $0.00 \pm 0.10$ & $0.22 \pm 0.13$ & $0.29 \pm 0.11$ \\
\hline \multicolumn{5}{|c|}{ Region C } \\
\hline $\mathrm{E}_{\mathrm{T}}^{\text {jets }}>140,100$, and $25 \mathrm{GeV}$ & $4.5 \pm 1.00$ & $0.00 \pm 0.10$ & $0.11 \pm 0.11$ & $0.39 \pm 0.13$ \\
\hline$\not_{T}>120 \mathrm{GeV}$ & $1.3 \pm 0.55$ & $0.00 \pm 0.10$ & $0.07 \pm 0.11$ & $0.01 \pm 0.09$ \\
\hline $\mathrm{H}_{\mathrm{T}} 330 \mathrm{GeV}$ & $0.67 \pm 0.39$ & $0.00 \pm 0.10$ & $0.07 \pm 0.11$ & $0.00 \pm 0.09$ \\
\hline
\end{tabular}

Tab. 4.12: Expected number of background events from Standard Model Z production processes. Only statistical uncertainties are shown. 


\begin{tabular}{|c|c|c|c|}
\hline Cuts & $t \bar{t}$ & WW & QCD \\
\hline Initial Exp. \# of Events & 2484 & 4653 & $9.53 \times 10^{6}$ \\
\hline Vertex: $\left|V_{z}\right|<60 \mathrm{~cm}$ & $2390 \pm 1.0$ & $4509 \pm 3.5$ & $2.59 \times 10^{6} \pm 1364$ \\
\hline$\geq 3$ jets $\left(E_{T}>25 \mathrm{GeV}\right.$ and $\left.\eta<2.0\right)$ & $2130 \pm 1.8$ & $1765 \pm 9.9$ & $864755 \pm 661$ \\
\hline 1 Central jet $(\eta<1.1)$ & $2119 \pm 1.8$ & $1709 \pm 9.8$ & $846405 \pm 652$ \\
\hline $\mathscr{E}_{T}>70 \mathrm{GeV}$ & $429 \pm 2.0$ & $86 \pm 2.7$ & $9517 \pm 55.8$ \\
\hline EEMF $>0.15$ & $429 \pm 2.0$ & $86 \pm 2.7$ & $9514 \pm 55.8$ \\
\hline $\mathrm{ECHF}>0.15$ & $427 \pm 1.9$ & $85 \pm 2.7$ & $9425 \pm 55.6$ \\
\hline$\Delta \phi\left(\mathbb{E}_{T}\right.$, jets $)$ & $295 \pm 1.7$ & $64 \pm 2.4$ & $261 \pm 10.2$ \\
\hline EMF of the jets & $222 \pm 1.5$ & $29 \pm 1.6$ & $242 \pm 9.8$ \\
\hline Minv $<76$ or Minv $>106 \mathrm{GeV} / c^{2}$ & $221 \pm 1.5$ & $29 \pm 1.6$ & $242 \pm 9.8$ \\
\hline$\Delta \phi\left(\mathscr{E}_{T}, \max\right.$ iso track $)$ & $156 \pm 1.3$ & $23 \pm 1.4$ & $234 \pm 9.7$ \\
\hline \multicolumn{4}{|c|}{ Region A } \\
\hline $\mathrm{E}_{\mathrm{T}}^{\mathrm{jets}}>95,55$, and $25 \mathrm{GeV}$ & $63.3 \pm 0.8$ & $3.2 \pm 0.5$ & $105.3 \pm 6.0$ \\
\hline $\mathscr{E}_{T}>75 \mathrm{GeV}$ & $56.2 \pm 0.8$ & $2.9 \pm 0.5$ & $83.1 \pm 5.4$ \\
\hline $\mathrm{H}_{\mathrm{T}}>230 \mathrm{GeV}$ & $43.0 \pm 0.7$ & $1.7 \pm 0.4$ & $71.1 \pm 4.9$ \\
\hline \multicolumn{4}{|c|}{ Region B } \\
\hline $\mathrm{E}_{\mathrm{T}}^{\text {jets }}>120,70$, and $25 \mathrm{GeV}$ & $25.5 \pm 0.5$ & $1.1 \pm 0.3$ & $52.7 \pm 4.0$ \\
\hline $\mathbb{E}_{T}>90 \mathrm{GeV}$ & $15.9 \pm 0.4$ & $0.7 \pm 0.3$ & $19.4 \pm 2.4$ \\
\hline $\mathrm{H}_{\mathrm{T}}>280 \mathrm{GeV}$ & $11.5 \pm 0.4$ & $0.5 \pm 0.2$ & $14.8 \pm 2.0$ \\
\hline \multicolumn{4}{|c|}{ Region C } \\
\hline $\mathrm{E}_{\mathrm{T}}^{\mathrm{jets}}>140,100$, and $25 \mathrm{GeV}$ & $8.8 \pm 0.3$ & $0.27 \pm 0.15$ & $26.4 \pm 2.6$ \\
\hline $\mathscr{E}_{T}>120 \mathrm{GeV}$ & $2.3 \pm 0.2$ & $0.09 \pm 0.09$ & $2.5 \pm 0.9$ \\
\hline $\mathrm{H}_{\mathrm{T}}>330 \mathrm{GeV}$ & $1.9 \pm 0.1$ & $0.09 \pm 0.09$ & $2.2 \pm 0.8$ \\
\hline
\end{tabular}

Tab. 4.13: Expected number of background events for $t \bar{t}$, WW and QCD multijet processes. Only statistical uncertainties are shown. 


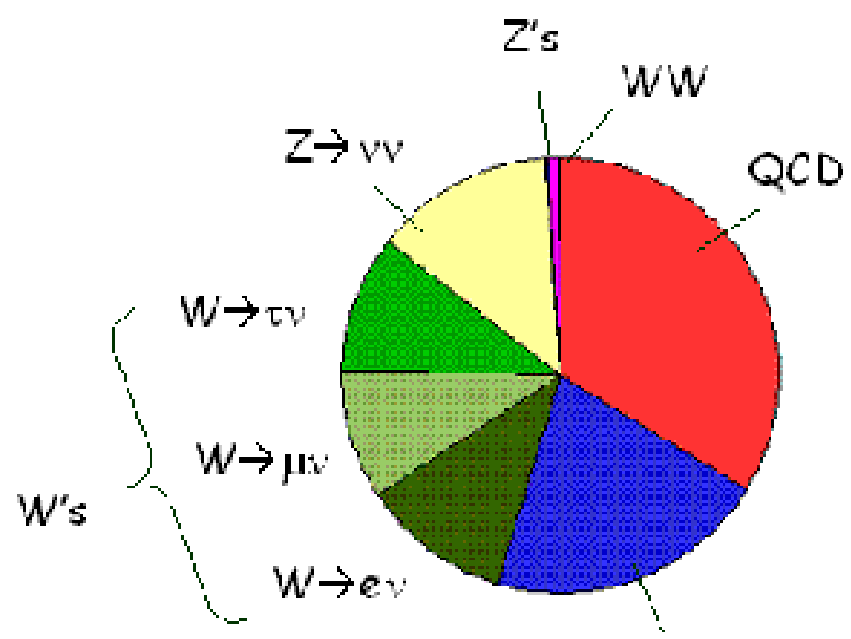

Top
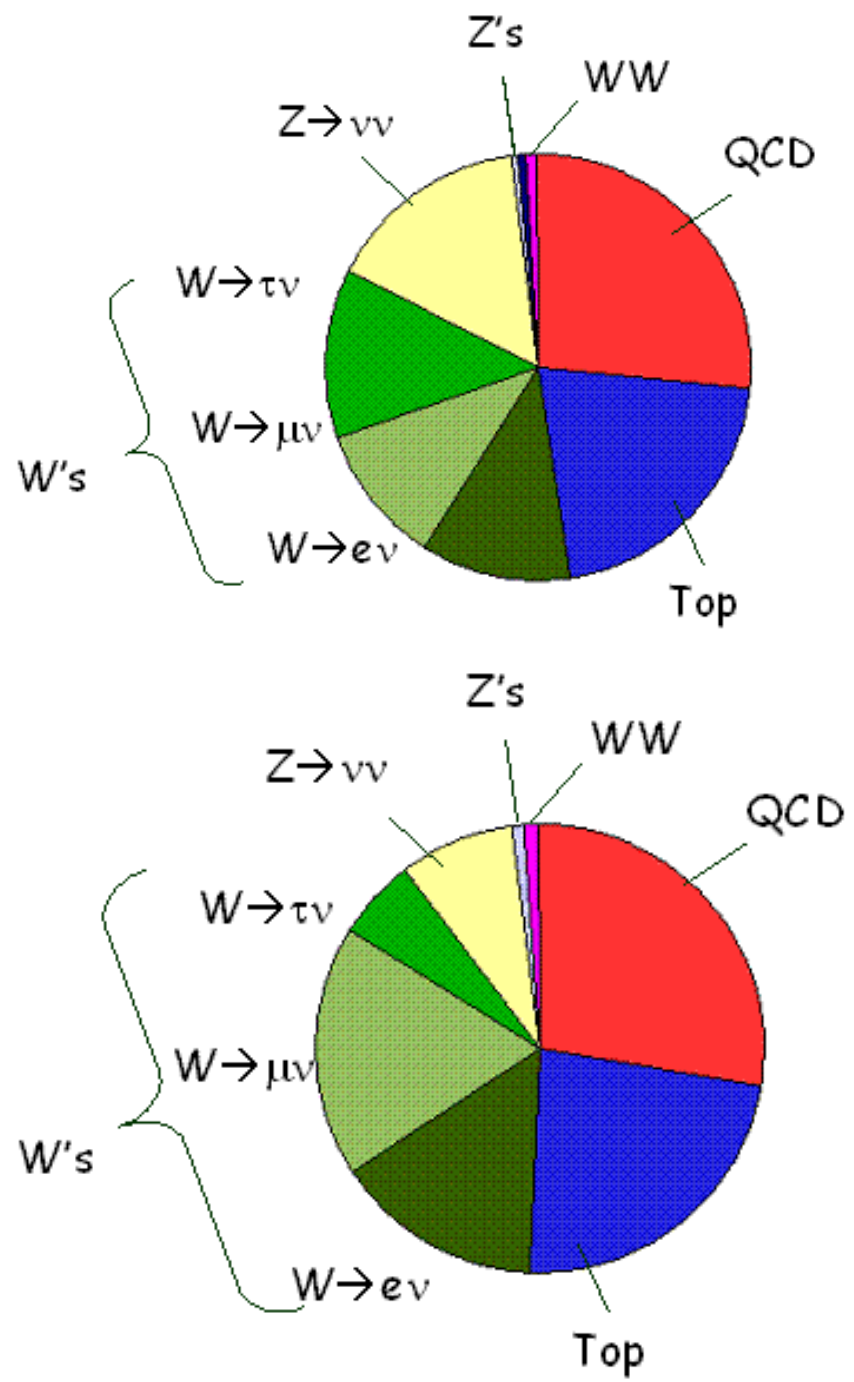

Fig. 4.34: Individual backgrounds relative contributions to the total one after all the cuts have been applied for Zone A (top), Zone B (middle), Zone C (low). 


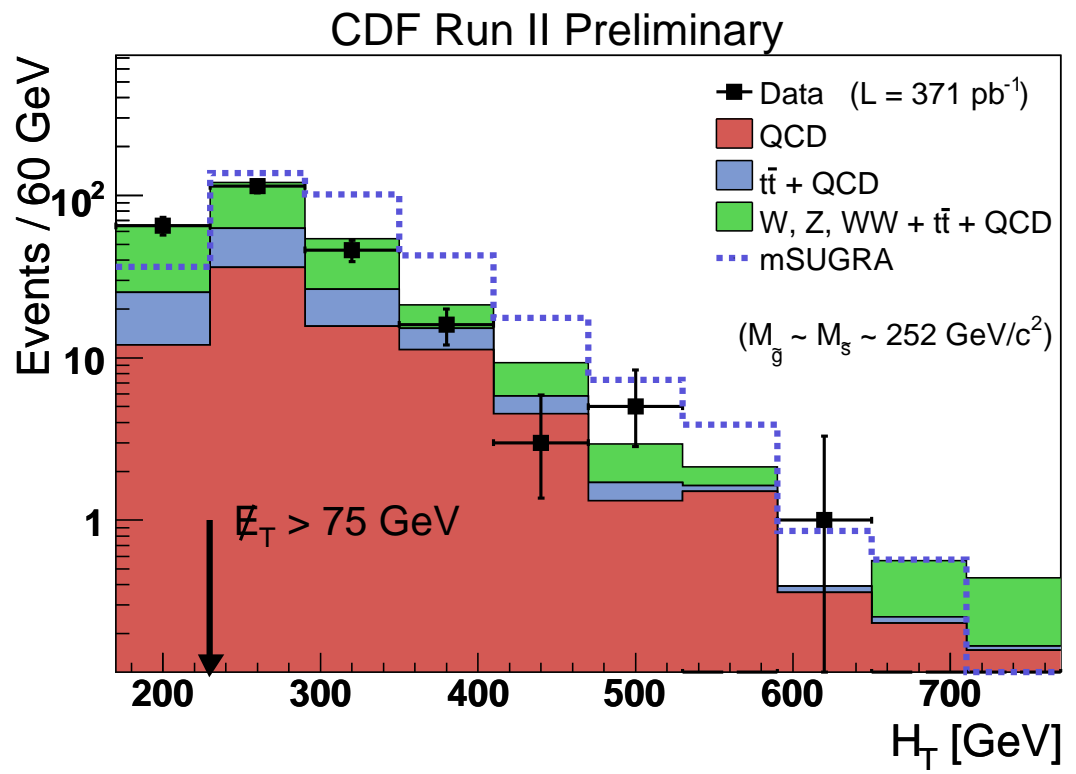

Fig. 4.35: $\mathrm{H}_{\mathrm{T}}$ distribution for the expected number of mSUGRA signal events, and background events in region A. The points correspond to the data events which pass the cuts for the region. Distributions have passed all the cuts except the $\mathrm{H}_{\mathrm{T}}$ one.

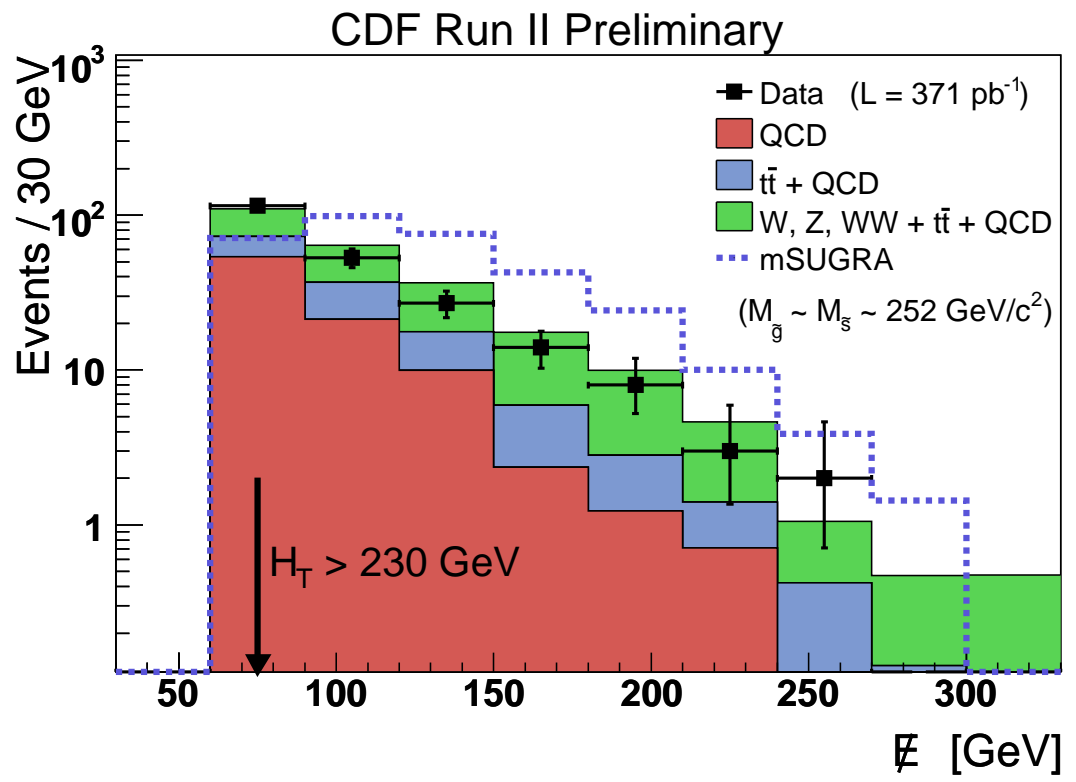

Fig. 4.36: $\mathbb{H}_{T}$ distribution for the expected number of mSUGRA signal events, and background events in region A. The points correspond to the data events which pass the cuts for the region. Distributions have passed all the cuts except the $\not_{T}$ one. 


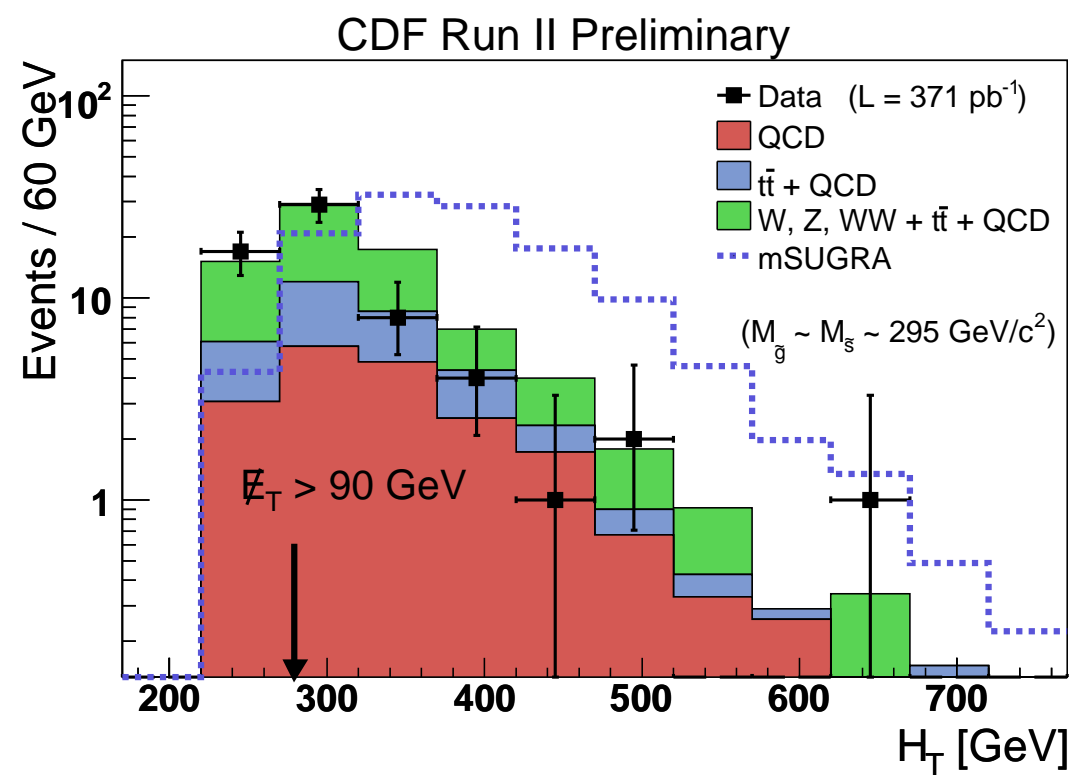

Fig. 4.37: $\mathrm{H}_{\mathrm{T}}$ distribution for the expected number of mSUGRA signal events, and background events in region $\mathrm{B}$. The points correspond to the data events which pass the cuts for the region. Distributions have passed all the cuts except the $\mathrm{H}_{\mathrm{T}}$ one.

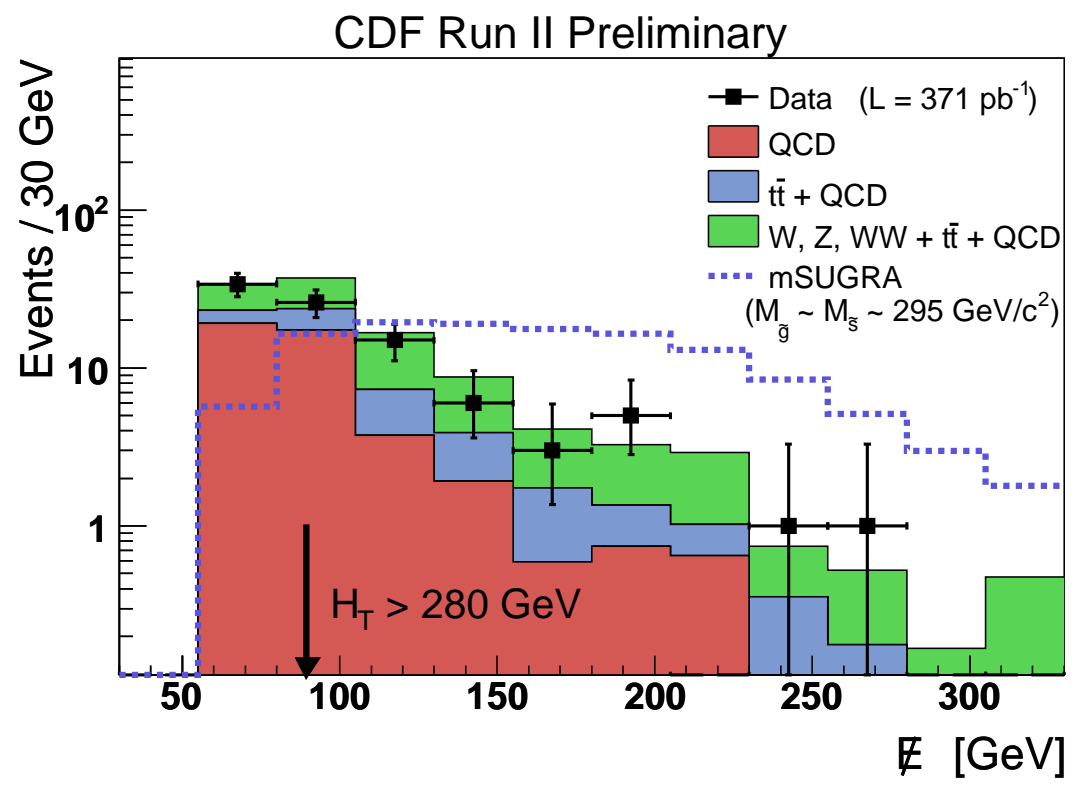

Fig. 4.38: $\not_{T}$ distribution for the expected number of mSUGRA signal events, and background events in region B. The points correspond to the data events which pass the cuts for the region. Distributions have passed all the cuts except the $\mathbb{H}_{T}$ one. 


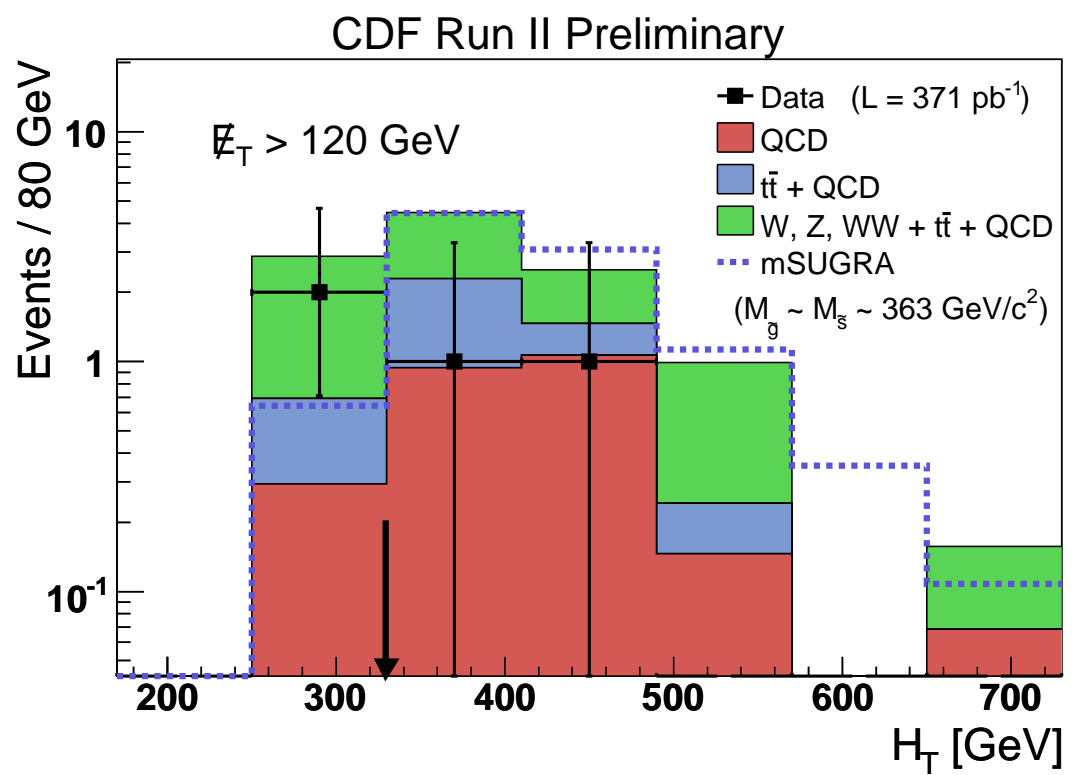

Fig. 4.39: $\mathrm{H}_{\mathrm{T}}$ distribution for the expected number of mSUGRA signal events, and background events in region $\mathrm{C}$. The points correspond to the data events which pass the cuts for the region. Distributions have passed all the cuts except the $\mathrm{H}_{\mathrm{T}}$ one.

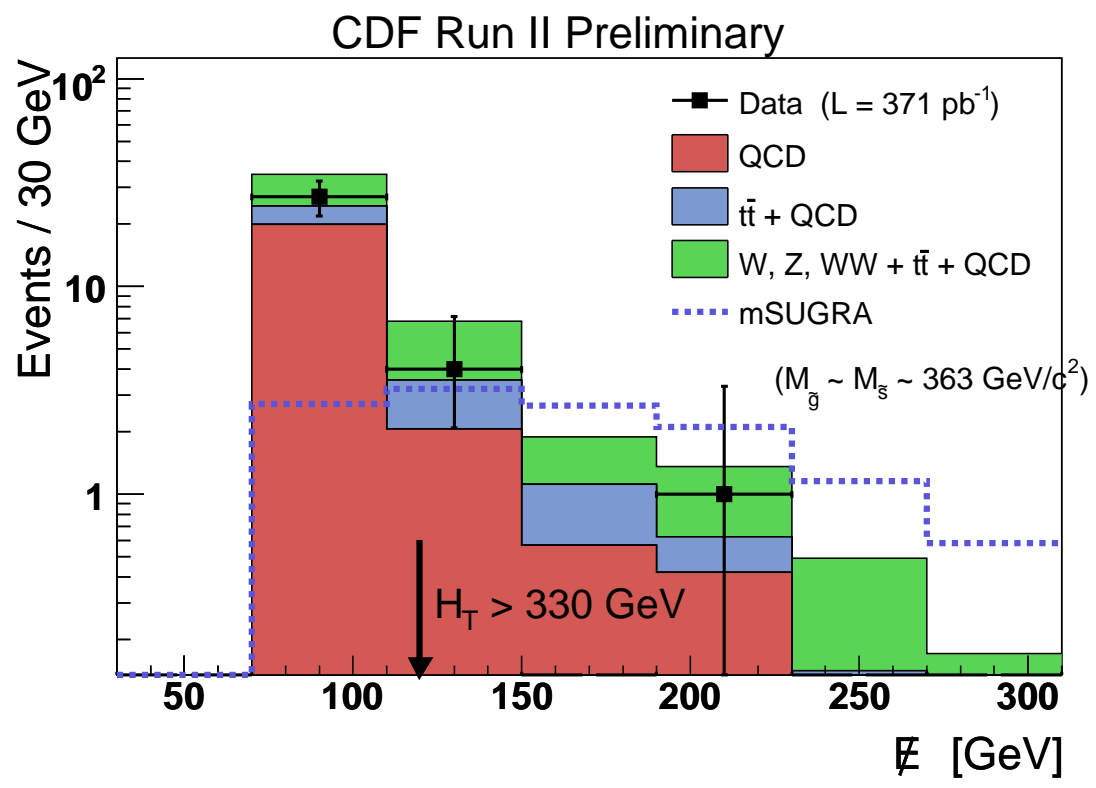

Fig. 4.40: $\mathbb{T}_{T}$ distribution for the expected number of mSUGRA signal events, and background events in region C. The points correspond to the data events which pass the cuts for the region. Distributions have passed all the cuts except the $\not_{T}$ one. 


\subsubsection{Highest energetic event}

From the two events found in Zone $\mathrm{C}$, the most energetic one is shown in Fig. 4.41. The upper plot is the $r-\phi$ view where the tracking system is clearly seen. The energy deposited in the calorimeter is shown in pink bars (electromagnetic) or blue bars (hadronic). The muon chambers are the outer part of the figure. To complement the view, the bottom figure shows a lego plot from an $\eta-\phi$ perspective of the deposited energy in the calorimeter. Here, the tower clustering of the different jets can be observed. The colours of the jets are defined to relate the jets from one plot to the other. The arrow on the upper plot represents the $\mathbb{E}_{T}$ direction.

This particular event contains three jets of energies above $25 \mathrm{GeV}\left(E_{T}^{1 \text { st }}=236 \mathrm{GeV}, E_{T}^{2 \text { nd }}=\right.$ $150 \mathrm{GeV}, E_{T}^{3 \mathrm{rd}}=84 \mathrm{GeV}$ ), with $\mathrm{H}_{\mathrm{T}}=470 \mathrm{GeV}$ and $\mathbb{E}_{T}=196 \mathrm{GeV}$.

\subsection{Limit Calculation}

Since no excess with respect to the Standard Model predictions was observed in the data, as can be seen from Tab. 4.9 and Tab. 4.10, as well as from Fig. 4.35 through Fig. 4.40, exclusion limits on gluino and squark production were stablished. Applying a Bayesian technique one can exclude a range of squark and gluino masses and production cross-sections to a 95\% confidence level (C.L). The Poisson probability of obtaining the observed result is:

$$
\frac{e^{-(s \varepsilon+b)}(s \varepsilon+b)^{n}}{n !},
$$

where $n$ is the number of observed events, $s$ is the cross section for our mSUGRA samples, $b$ is the number of expected background and $\varepsilon$ is the product of the acceptance times luminosity, and has units of inverse cross section.

In the Bayesian approach, a prior function need to be defined. Assuming a flat function $\pi(s)=1$ for $s \geq 0$ and $\pi(s)=0$ for $s<0$, the upper limit $s_{u}$ at confidence level $\beta$ is computed, in a finite Bayesian prior-ensemble approximation, by solving:

$$
I\left(s_{u}\right)=(1-\beta) I(0)
$$

where $I\left(s_{0}\right)$ is the integral:

$$
I(0)=\frac{1}{M} \sum_{i=1}^{M}\left[\int_{s_{0}}^{\infty} \frac{e^{-(s \varepsilon+b)}(s \varepsilon+b)^{n}}{n !} d s\right],
$$

with $M$ being the number of random pairs of $\varepsilon$ and $b$ events in this finite approach. 

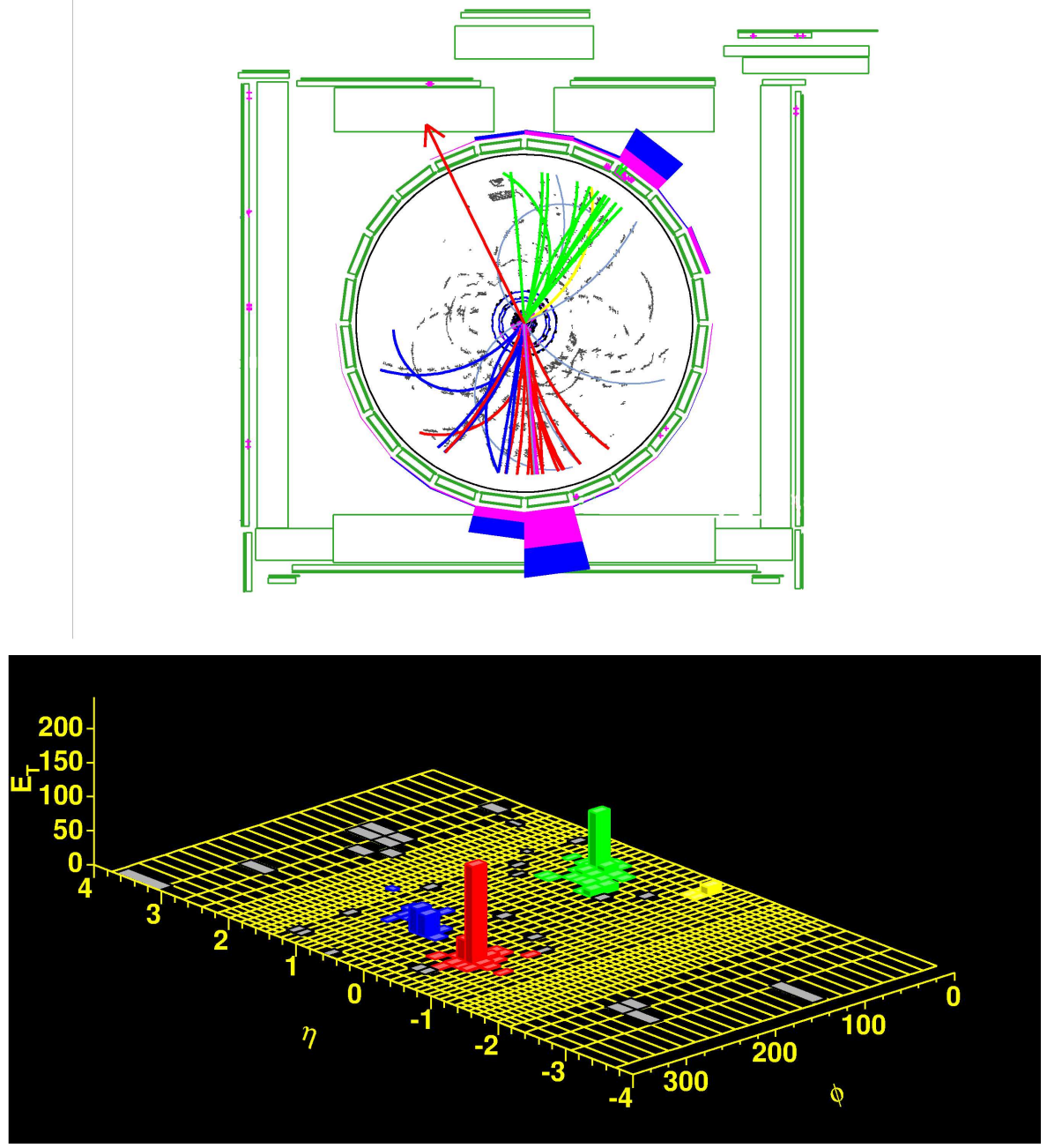

Fig. 4.41: CDF central tracking chamber and calorimeter $(r-\phi$ view) (top) and Calorimeter "Lego" plot (bottom) for the event with the highest $E_{T}$ and $\mathrm{H}_{\mathrm{T}}$. This event has three jets above $25 \mathrm{GeV}\left(E_{T}^{1 \text { st }}=236 \mathrm{GeV}, E_{T}^{2 \text { nd }}=\right.$ $150 \mathrm{GeV}, E_{T}^{3 \mathrm{rd}}=84 \mathrm{GeV}$, with $\mathrm{H}_{\mathrm{T}}=470 \mathrm{GeV}$ and $\not_{T}=196 \mathrm{GeV}$.

This procedure and the program that implements it is described thoroughly in the note [79]. This program allows the use of correlated and uncorrelated uncertainties into the limit calculation process as well as a choice from different random generators. Due to the fact that the theoretical uncertainties on the cross-section can be close to $30 \%$ as shown in Tab. 4.5, a gamma function was chosen as a random generator in order to avoid non-converging integrals. The statistical and the ISR/FSR uncertainties are considered uncorrelated. The rest of the systematics (renormalisation scale, PDF, jet energy scale and luminosity) are considered correlated. The PDF and the renormalisation scale uncertainties that affect the signal cross-section are properly translated into signal acceptance uncertainties and introduced into the limit calculation. All systematic contributions are quoted as 1 sigma uncertainties except for the PDF which corresponds to 1.64 sigmas [80]. 
A poissonian fluctuation is used to calculate the expected cross section using the following formula:

$$
\sigma_{\text {exp }}=\sum P\left(n_{\text {obs }}^{i} \mid n_{\text {exp }}\right) * \sigma_{o b s}^{i}
$$

where $\sigma_{o b s}$ is the cross section upper limit with the number of observed events, $n_{o b s}, P\left(n_{\text {obs }}^{i} \mid n_{\text {exp }}\right)$ is the poisson probability of observing $n_{o b s}$ events when the mean value is the number of expected background events, $n_{\text {exp }}$, and the sum is over all the possible values that give a significant contribution to this poisson fluctuation. In any case, for numbers larger or similar to 10 , it is a good approximation to use $n_{o b s}=n_{\text {exp }}$, instead.

Since three set of cuts were defined, three different expected limits can be stablished for each point. Thus, each point is associated to a single set of cuts which is the one that gives the highest expected limits. Afterwards, the observed limits are calculated with this criteria unchanged.

1.5 million $(\varepsilon, b)$ pairs of random events were generated to evaluate the integrals and estimate the maximum cross section for each of the points in the mSUGRA plane. The results in terms of cross sections are shown in Fig. 4.42 and Fig. 4.43. Since the theoretical uncertainties are properly taken into account inside the limit calculation, the crossing between the 95\% C.L. cross section with the nominal cross section gives an upper estimation for the squarks/gluino masses along the first column of points generated $\left(M_{\tilde{g}} \sim 185 \mathrm{GeV} / \mathrm{c}^{2}\right)$ or along the diagonal $\left(M_{\tilde{q}} \sim M_{\tilde{g}}\right)$, respectively.

The result of this procedure is a set of points which are either excluded or not. In order to determine the excluded region independently from our generation set, a smooth line is linearly interpolated between any pair of points on the boundary regions. For each of these points, the ratio $r=\sigma_{95} / \sigma$ was computed so that excluded points have $r<1$ and not excluded points $r>1$, being $r^{*} \equiv r=1$ the point that lies on the exclusion line. Then, between every pair of excluded (E) and not excluded (NE) points, the corresponding squark/gluino masses of the intermediate point $\left(m^{*}\right)$ are extracted by solving equations of type:

$$
r^{*}=r_{E}+\frac{r_{N E}-r_{E}}{m_{N E}-m_{E}} \cdot\left(m^{*}-m_{E}\right)=1,
$$

where $m_{E}$ and $m_{N E}$ are the masses of the excluded and not excluded points, respectively.

In Fig. 4.44, the previous exclusion regions determined by other experiments are also presented. Since in this analysis the observed events are less than the expectation, the observed excluded area is bigger than the expected one. Topologies involving three jets in the final state are specially important close to the diagonal, where the production of one squark and one gluino dominates, and it is where higher masses are excluded with this 3-jets analysis. The drop below the diagonal is mainly due to the fact that squark pair-production dominates and hence, 2- 
jets final states dominate this portion of the plane. The region of low gluino masses and high squark masses is very sensitive to the systematic effects provided that the crossing between the observed/expected lines with the nominal cross-sections is very shallow, as shown in Fig. 4.42.

In the region where $M_{\tilde{g}} \approx M_{\tilde{q}}$, masses up to $380 \mathrm{GeV} / \mathrm{c}^{2}$ at $95 \%$ C.L are excluded. In any case, the limit $M_{\tilde{g}}>220 \mathrm{GeV} / \mathrm{c}^{2}$ holds.
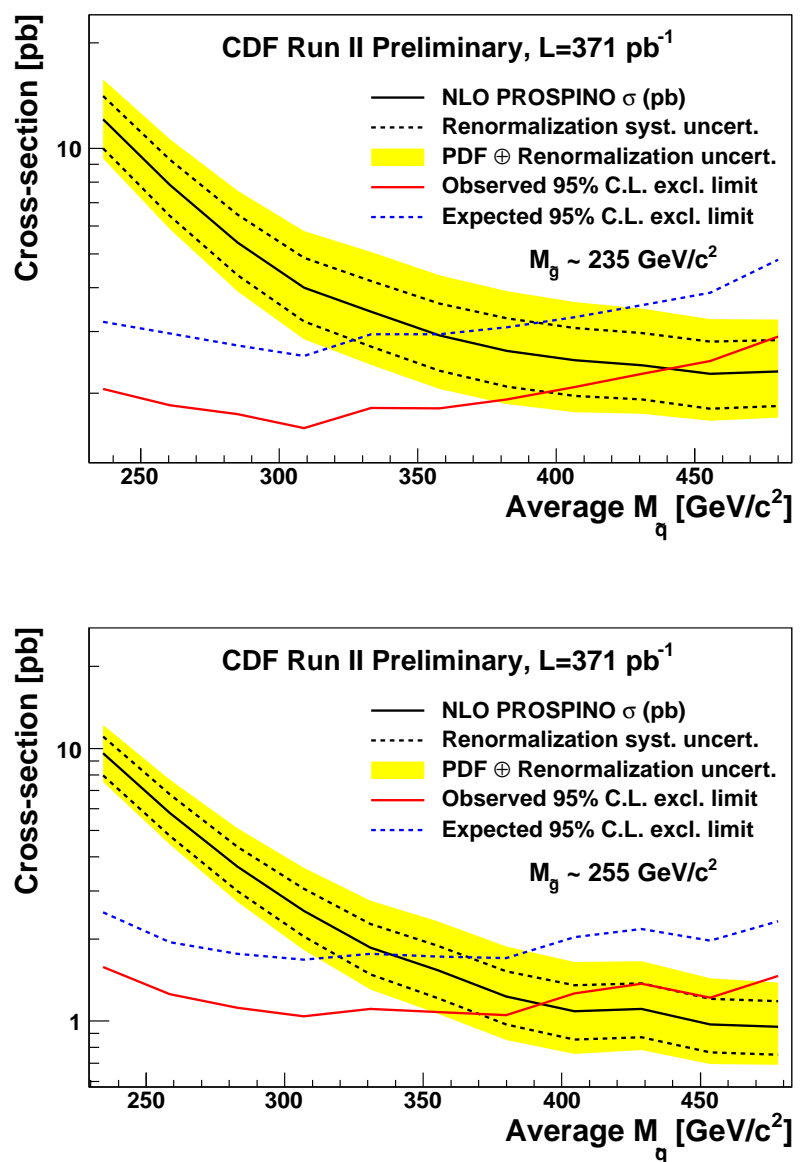

Fig. 4.42: Cross section as a function of squark mass for two $\mathrm{M}_{\tilde{g}}$ values (columns). The observed and expected limits at $95 \%$ C.L. are also shown. The yellow band shows the total effect of the PDFs and the renormalisation scale uncertainties. 


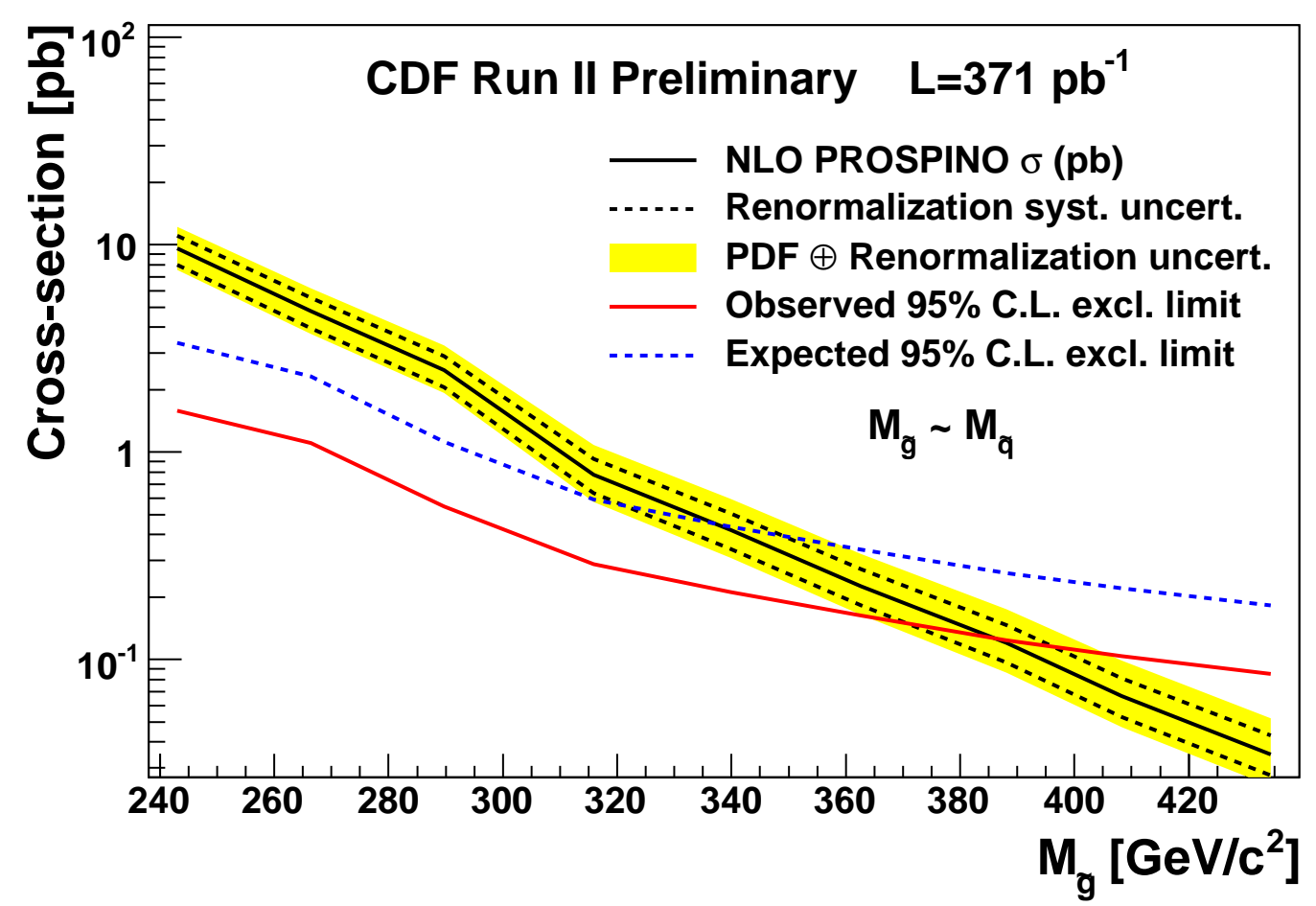

Fig. 4.43: Cross section as a function of gluino/squark masses in the case of $\mathrm{M}_{\tilde{\mathrm{q}}} \approx \mathrm{M}_{\tilde{\mathrm{g}}}$ (diagonal). The observed and expected limits at $95 \%$ C.L. are also shown. The yellow band shows the total effect of the PDFs and the renormalisation scale uncertainties. 


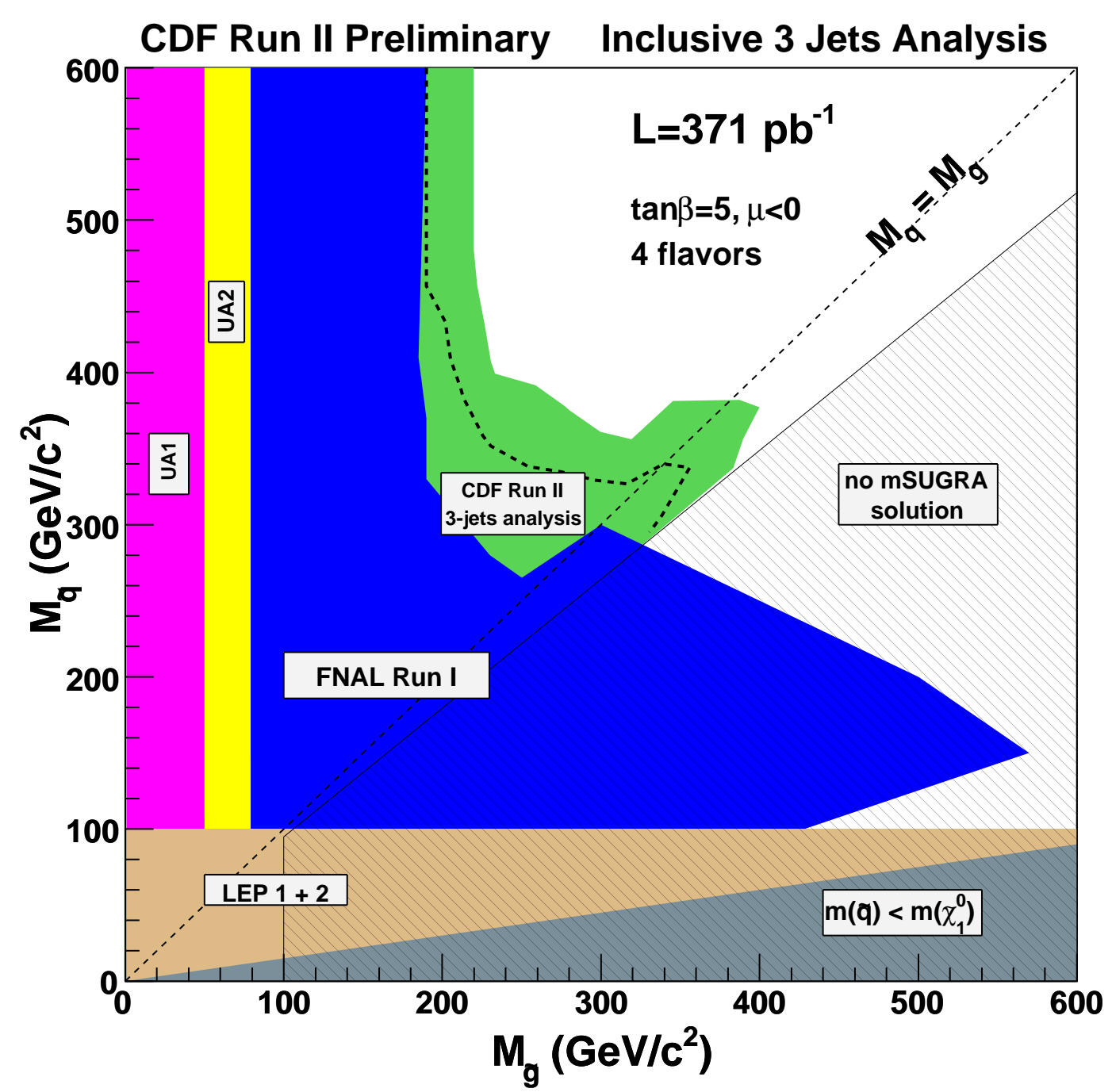

Fig. 4.44: $\mathrm{M}_{\tilde{\mathrm{q}}}-\mathrm{M}_{\tilde{\mathrm{g}}}$ exclusion plot at the $95 \%$ C.L in the framework of mSUGRA assuming R-parity conservation. The pink region was excluded by the UA1 experiment [52]. The yellow region was excluded by the UA2 experiment [53]. The blue region was excluded by the CDF and DØ experiments after Run I [51]. The brown region was excluded by the LEP experiment [81]. In the gray region, the squark mass is lower than the mass of the lightest neutralino. In the black hashed region there is no mSUGRA solution. The green region shows the area excluded by the present analysis with $371 \mathrm{pb}^{-1}$ of CDF Run II data. 



\section{Chapter 5}

\section{Conclusions and Future Prospects}

In this thesis, the results of the search for squarks and gluinos in multiple jets plus missing transverse energy final states have been presented. No evidence of these new particles have been found in $371 \mathrm{pb}^{-1}$ of CDF Run II data. New limits have been set which exclude gluino masses below $220 \mathrm{GeV}$ and, in the region where $M_{\tilde{g}} \approx M_{\tilde{q}}$, masses below $380 \mathrm{GeV} / \mathrm{c}^{2}$ are excluded. These limits are valid in a mSUGRA scenario with $\tan \beta=5, A=0$ and $\mu<0$ assuming the lightest four squark flavours degenerate in mass.

To obtain these results a careful study of the beam conditions and their contribution to events with $\mathscr{E}_{T}$ final states has been performed. Special attention has been taken in studying the different SM backgrounds and their normalisations at NLO. Dedicated cuts have been introduced to remove the background processes and main discriminating variables have been optimised for different signal regions. The different systematic uncertainties have also been considered.

This is the first time that this search is performed at CDF Run II and the results presented here show significant improvements with respect to the constraints from previous experiments. Thus, this analysis has established the procedure to continue searching for squarks and gluinos with the new data samples that CDF is collecting from Tevatron. Some improvements may also be implemented by considering other hadron final states with different jet multiplicities. This could help extending the sensitivity of the analysis to regions where gluino and squark masses are not similar.

At the forthcoming LHC, the search for squarks and gluinos in this inclusive channel constitutes one of the first analyses to be performed. The $\mathbb{E}_{T}$ and multiple jets final states are present in multiple decay modes of many models beyond the SM. The experience from Tevatron in working on an hadron collider environment will be useful for these kind of studies aiming to discover the presence of supersymmetric processes. 



\section{Bibliography}

[1] F. Halzen, A.D. Martin, Quarks and Leptons: An Introductory Course in Modern Particle Physics, Wiley Text Books (1984).

[2] W.M. Yao et al., J. Phys. G33, 1 (2006) (For last updates see: http://pdg.lbl.gov/pdg.html)

[3] S. Glashow, Nucl. Phys. B22 (1961) 579;

S. Weinberg, Phys. Rev. Lett. 19 (1967) 1264;

A. Salam, in Elementary Particle Theory, ed. N. Svartholm, Almquist and Forl, Stockholm (1968).

[4] L.D. Faddeev and V.N. Popov, Phys. Lett. B25 (1967) 29.

[5] G. Arnison et al. [UA1 Collaboration], Phys. Lett. B122 (1983) 103;

G. Arnison et al. [UA1 Collaboration], Phys. Lett. B126 (1983) 398.

[6] R.K. Ellis, W.J. Stirling, B.R. Webber, QCD and Collider Physics, (Cambridge 1996).

[7] S. Eidelman et al. [Particle Data Group Collaboration], Phys. Lett. B592, (2004) 1 [http://pdg.lbl.gov].

[8] V.N. Gribov, L,N. Lipatov. Sov.J.Nucl.Phys. 15 (1972) 438; G. Altarelli, G. Parisi. Nucl. Phys. B126 (1977) 298; Yu.L. Dokshitzer Sov.Phys. JETP 46 (1977) 641.

[9] J. Pumplin, D.R. Stump, J. Huston, H.L. Lai, P. Nadolsky, and W.K. Tung, J. High Energy Phys. 07, (2002) 012. [arXiv:hep-ph/0201195].

[10] M. Bengtsson, T. Sjöstrand, Z. Phys. C37 (1988) 465.

[11] B. Andersson, G. Gustafson and B. Söderberg, Z. Phys. C20 (1983) 317, Nucl. Phys. B264 (1986) 29.

B. Andersson, G. Gustafson, G. Ingelman and T. Sjöstrand, Phys. Rep. 97 (1983) 33.

[12] B.R. Webber, Nucl. Phys. B238 (1984) 492. 
[13] D. Amati and G. Veneziano, Phys. Lett. B83 (1979) 87;

A. Bassetto, M. Ciafaloni and G. Marchesini, Phys. Lett. B83 (1979) 207;

G. Maschesini, L. Trentadue and G. Veneziano, Nucl. Phys. B181 (1980) 335.

[14] H. Georgi, H. Quinn, and S. Weinberg, Phys. Rev. Lett. 33 (1974) 451.

[15] ALEPH, DELPHI, L3 and OPAL Collaborations, Phys. Lett. $B 565$ (2003) 61 [arXiv:hepex/0306033].

[16] J. Erler, P. Langacker, Phys. Lett. B592 (2004) [arXiv:hep-ph/0407097].

[17] B. Lee, C. Quigg and H. Thacker, Phys. Rev. D16 (1977) 1519.

[18] L. Susskind, Phys. Rev. D20 (1979) 2619.

[19] Stephen P. Martin, arXiv:hep-ph/9709356.

[20] Hitoshi Murayama, arXiv:hep-ph/0002232.

[21] R. Haag, J. Lopuszanski and M. Sohnius, Nucl. Phys. B88 (1975) 416.

[22] R. Haag, J. Lopuszánski and M. Sohnius, Nucl. Phys. B88 (1975) 257.

[23] J. Polchinski, 1. Susskind, Phys. Rev. D26 (1982) 3661.

[24] L. Maiani, Proc. Summer School on Particle Physics, Gif-sur-Yvette, 1979 (IN2P3, Paris, 1980) p.3;

G't Hooft et al., eds. Recent Developments in Field Theories (Plenum Press, new York, 1980;

E. Witten, Nucl. Phys. B188 (1981) 513;

R.K. Kaul, Phys. Lett. B109 (1982) 19.

[25] U. Amaldi, W. de Boer and H. Furstenau, Phys. Lett. B260 (1991) 447

[26] U. Amaldi, W. de Boer, P.H. Frampton, H. Furstenau and J.T. Liu. Phys. Lett. B281 (1992) 374.

[27] P. Langacker and M. Luo, Phys. Rev. D44 (1991) 817;

U. Amaldi, W. de Boer and H. Furstenau, Phys. Lett. B260 (1991) 447;

J. Ellis, S. Kelley and D.V. Nanopoulos, Phys. Rev. B260 (1991) 131.

[28] F. Gliozzi, J. Scherk and D. Olive, Nucl. Phys. B122 (1977), 253.

[29] S. Heinemeyer, W. Hollik, D. Stöckinger, A.M. Weber and G. Weiglein, arXiv:hep$\mathrm{ph} / 0604147$. 
[30] CDF Collaboration, DØ Collaboration and Tevatron Electroweak Working Group, arXiv:hep-ph/0608032.

[31] H. E. Haber, in Recent Directions in Particle Theory, Proceedings of the 1992 Theoretical Advanced Study Institute in Elementary Particle Physics, eds. J. Harvey and J. Polchinski (World Scientific, Singapore, 1993) pp. 589-686.

[32] D. Gross and R.Jackiw, Phys. Rev. D6 (1972) 477; C. Bouchiat, J. Iliopoulos and P. Meyer, Phys. Lett. $B 38$ (1972) 519; H. Georgi and S. Glashow, Phys. Rev. D6 (1972) 429; L. Alvarez-Gaume and E. Witten, Nucl. Phys. B234 (1983) 269.

[33] J.F. Gunion, H. E. Haber, G. Kane, S. Dawson, The Higgs hunter's guide, Addison Wesley (1990) Chapter 4.

[34] S. Dawson, arXiv:hep-ph/9712464.

[35] S. Dimopoulos and H. Georgi, Nucl. Phys. B193 (1981) 150; N. Sakai, Z. Phys. C11 (1981) 153; P. Fayet, Phys. Lett. B69 (1977) 489 [Phys. Lett. B84 (1979) 416].

[36] S. Weinberg, Phys. Rev. D26 (1982) 287; N. Sakai and T. Yanagida, Nucl. Phys. B197 (1982) 533; S. Dimopoulos, S. Raby, and F. Wilczek, Phys. Lett. B112 (1982) 133; J. Ellis, D. Nanoupoulos and S. Rudaz, Nucl. Phys. B202 (1982) 43.

[37] G. Farrar and P. Fayet, Phys. Lett. B76 (1987) 575; F. Zwirner, Phhys. Lett. 132B (1983) 103; L. Hall and M. Suzuki, Nucl. Phys. B231 (1984) 419;

J. Ellis, G. Gelmini, C. Jarlskog, G. Ross, and J. Valle, Phys. Lett. B150 (1985) 142;

G. Ross and J. Valle, Phys. Lett. B151 (1985) 375;

S. Dawson, Nucl. Phys. B261 (1985) 297;

S. Dimopoulos and L. Hall, Phys. Lett. B207 (1988) 210.

[38] C. Carlson, P. Roy, and M. Sher, Phys. Lett. B357 (1995) 99.

[39] G. Bhattacharyya, arXiv:hep-ph/9608415.

[40] D. J. H. Chung, L. L. Everett, G.L. Kane, S. F. King, J. Lykken and Lian-Tao Wang, Phys. Rept. 407 (2005) 1.

[41] H. P. Nilles, Phys. Rept. Vol.110, Issues 1-2, August 1984, pages 1-162.

For general SUGRA models and further citations see: Pran Nath, arXiv:hep-ph/0307123.

[42] L.E. Ibáñez and C. López, Nucl. Phys. B233 (1984) 511; L.E. Ibáñez and C. López, Nucl. Phys. B256 (1985) 218.

[43] M. Carena, S. Pokorski, M. Olechowski, C.E.M. Wagner, Nucl. Phys. B426 (1994) 269 [arXiv:hep-ph/9402253]. 
[44] G.L. Kane, Chris Kolda, Leszek Roszkowski, James D. Wells, arXiv:hep-ph/9312272.

[45] F. Gianotti, arXiv:hep-ph/0112013; A. Lipniacka, arXiv:hep-ph/0112280.

[46] H. Baer, C. Chen, F. Paige, X. Tata, G.L. Kane, C. Kolda, L. Roszkowski, J.D. Wells, Phys. Rev. D54 (1996) 5866.

[47] V. Barger, C.E.M. Wagner et al. Report of the SUGRA Working Group for Run II of the Tevatron hep-ph/0003154, (2000).

[48] W. Beenakker, R. Höpker, M. Spira and P. M. Zerwas, Nucl. Phys. B492 (1997) 51 [arXiv:hep-ph/9610490].

[49] G.L. Kane and J.P. Leveille, Phys. Lett. B112 (1992) 227;

P.R. Harrison and C.H. Llewellyn Smith, Nucl. Phys. B223 (1983) 542;

E. Reya and D.P. Roy, Phys. Rev. D32 (1985) 645;

S. Dawson, E. Eichten and C. Quigg, Phys. Rev. D31 (1985) 1581;

H. Baer and X. Tata, Phys. Lett. B160 (1985) 159.

[50] W. Beenakker, R. Höpker, M. Spira and P.M. Zerwas, Phys. Rev. Lett. 74 (1995) 2905;

W. Beenakker, R. Höpker, M. Spira and P.M. Zerwas, Z. Phys. C69 (1995) 163.

[51] DØ Collaboration, B. Abbott et al., Phys. Rev. Lett. 834937 (1999). CDF Collaboration, T. Affolder et al., Phys. Rev. Lett. 88 (2002) 041801.

[52] UA1 Collaboration, C. Albajar et al., Phys. Lett. B198 (1987) 261.

[53] UA2 Collaboration, S. Albachi et al., Phys. Lett. B235 (1990) 363.

[54] Fermilab Beam Division, http://www-bd.fnal.gov/

[55] CDF experiment, http://www-cdf.fnal.gov/

[56] F. Abe et al. [The CDF Collaboration], Phys. Rev. Lett. 74, (1995) 2626.

[57] R. Blair et al. [CDF-II Collaboration], The CDF-II detector: Technical design report, FERMILAB-PUB-96-390-E.

[58] A. Sill et al., Nucl. Instrum. Meth. A447, (2000) 1-8 .

[59] T.Affolder et al., Nucl. Instrum. Meth. A526 (2004) 249.

[60] D. Acosta et al., Nucl. Instrum. Meth. A518, (2004) 605.

[61] L. Balka et al., Nucl. Instrum. Meth. A267, (1988) 272.

[62] S. Bertolucci et al., Nucl. Instrum. Meth. A301, (1988) 267. 
[63] G. Apollinari et al., Proceedings of the Fourth International Conference on Calorimetry in High Energy Physics, World Scientific, Singapore, p. 200, (1994).

[64] S. Kuhlmann et al., Nucl. Instrum. Meth. A518, (2004) 39-41.

[65] G. Apollinari et al., Nucl. Instrum. Meth. A412, (1998) 515-526.

[66] F. Abe et al., Phys. Rev. Lett. 73, (1994) 2662.

[67] M. Albrow et al., Nucl. Instrum. Meth. A431 (1999) 104.

[68] C. M. Ginsburg, The CDF Collaboration, FERMILAB-CONF-03/386-E. Published Proceedings International Europhysics Conference on High-Energy Physics (HEP 2003), Aachen, Germany, July 17-23, 2003.

[69] D. Acosta et al. [CDF Collaboration], The CDF Cherenkov luminosity monitor, Nucl. Instrum. Meth. A461, (2001) 540.

[70] CDF-7446 Lum. Group, Luminosity Uncertainty for Run II up until August 2004; S. Klimenko et al., FERMILAB-FN-0741 (2003); D. Acosta et al., Nucl. Instrum. Meth. A494, (2002) 57.

[71] H. Baer, F.E. Paige, S.D. Protopopescu, and X. Tata, ISAJET 7.48: A Monte Carlo Event Generator for $p p, p \bar{p}$, and $e^{+} e^{-}$Reactions, arXiv:hep-ph/0001086.

[72] T. Sjöstrand et al. Computer Phys. Commun. 135 (2001).

[73] W. Beenakker, R. Höpker, and M. Spira, PROSPINO: A Program for the Production of Supersymmetric Particles in Next-To-Leading Order QCD.

[74] J, Campbell, and R. K. Ellis, MCFM: A Monte Carlo for FeMtobarn processes at Hadron Colliders.

[75] M. Cacciari, S. Frixione, M. L. Mangano, P. Nason, and G. Ridolfi, arXiv:hep/ph-0303085.

[76] J. Campbell, and R. K. Ellis Phys. Rev. D64 (2002), arXiv:hep-ph/0202176.

[77] A. Abulencia et al., Phys. Rev. Lett. 96 (2006) 122001.

[78] D. Sutmp, J. Huston, J. Pumplin, and W. Tung, arXiv:hep-ph/0303013;

A.D. Martin, R. G. Roberts, W. J. Stirling, and R.S. Thorne, arXiv:hep/ph-0211080;

J. Pumplin, D. Stump, R. Brock, D. Casey, and J. Huston, arXiv:hep-ph/0101032.

[79] Joel Heinrich, CDF Note 7587.

[80] J. Pumplin, D.R. Stump, J. Huston, H.L. Lai, P. Nadolsky, W.K. Tung, arXiv:hepph/0201195; JHEP 0207 (2002) 012. 
[81] The LEP-SUSY working group: http://lepsusy.web.cern.ch/lepsusy/

[82] G. C. Blazey et al., arXiv:hep-ex/0005012. 


\section{Appendix A}

\section{Data Quality Monitoring}

\section{A.1 Introduction}

The Data Quality Monitoring (DQM) system performs checks while data are being taken and serves as an "early warning system". In addition, further statistical tests are performed offline, after the data have been processed. Therefore, each run in the data-taking has a set of binary decisions associated with the results from the online and offline checks of the different subdetectors. The information is provided to each subsystem expert for a final validation. Since physics analyses may be only interested in the behaviour of given subdetectors, specialised lists, called "GoodRunLists", are provided to the different physics groups.

In the following sections, the discussion is focused on the offline part of the system, for which I made major contributions.

\section{A.2 The offline DQM system}

In the offline DQM system, a number of observables are chosen to monitor the behaviour of the different detector subsystems: tracking, calorimeter and muon chambers. In addition, some histograms related to higher level objects (electrons, photon, jets, impact parameter, $\mathrm{J} / \Psi$, primary vertex) are used to check the offline reconstruction itself. Tests are performed after data are fully processed with final calibration constants and beam-lines. For long runs, the histograms are also visually inspected by an "offline shifter". 


\section{A.2.1 Tracking}

The hit residuals spread for each of the eight super-layers are the relevant distributions for COT monitoring since deviations can be associated to noise in the COT or problems in the tracking fitter. These distributions for first and last super-layers are shown in Fig. A.1. As monitoring criteria, a mean value not significantly $(6 \sigma)$ greater than $20 \mu \mathrm{m}$ and a RMS not significantly greater than $190 \mu \mathrm{m}$ are required. Some distributions on the measured $\mathrm{dE} / \mathrm{dx}$ for the different COT hits are also used.
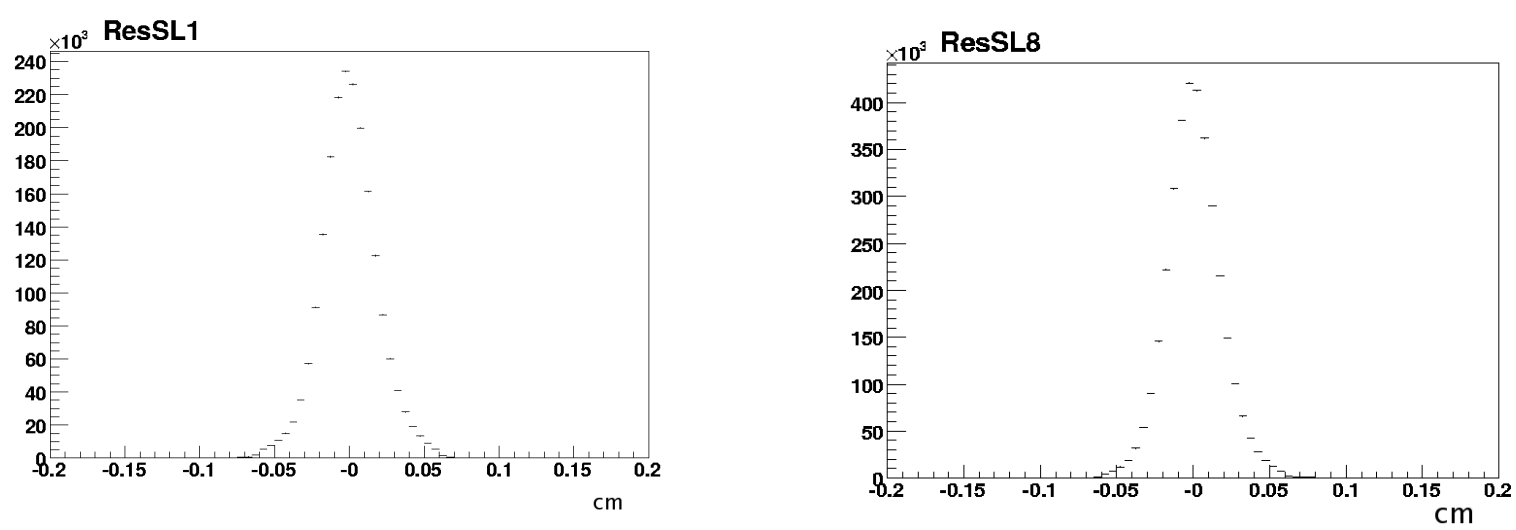

Fig. A.1: Hit residual distributions for SL1 (left) and SL8 (right) for a particular run.

These checks are performed using a specific set of data processed with the highest priority (just after the beamlines are available). In this way, first checks on data can be performed within a week after data-taking. Once all final calibrations are available, analogous tests are performed using other type of data sets, like minimum bias samples.

Beam-lines are monitored using the correlation between the average impact parameter and the azimuthal direction, as extracted from tracks, since a mistake in the beam-lines would translate into a clear $\phi$ modulation. In addition, tracking efficiency distributions are also checked for Silicon monitoring.

\section{A.2.2 Calorimeter}

The calorimeter performance is monitored using the tower occupancies. As shown in Fig. A.2 there are two distributions for each type of calorimeter (electromagnetic and hadronic). Each distribution have a low $(500 \mathrm{MeV})$ and a high $(1 \mathrm{GeV})$ transverse energy threshold to study possible towers abnormally cold or hot, respectively.

The values from all the towers of a certain $\eta$ value are averaged, assuming the response is 

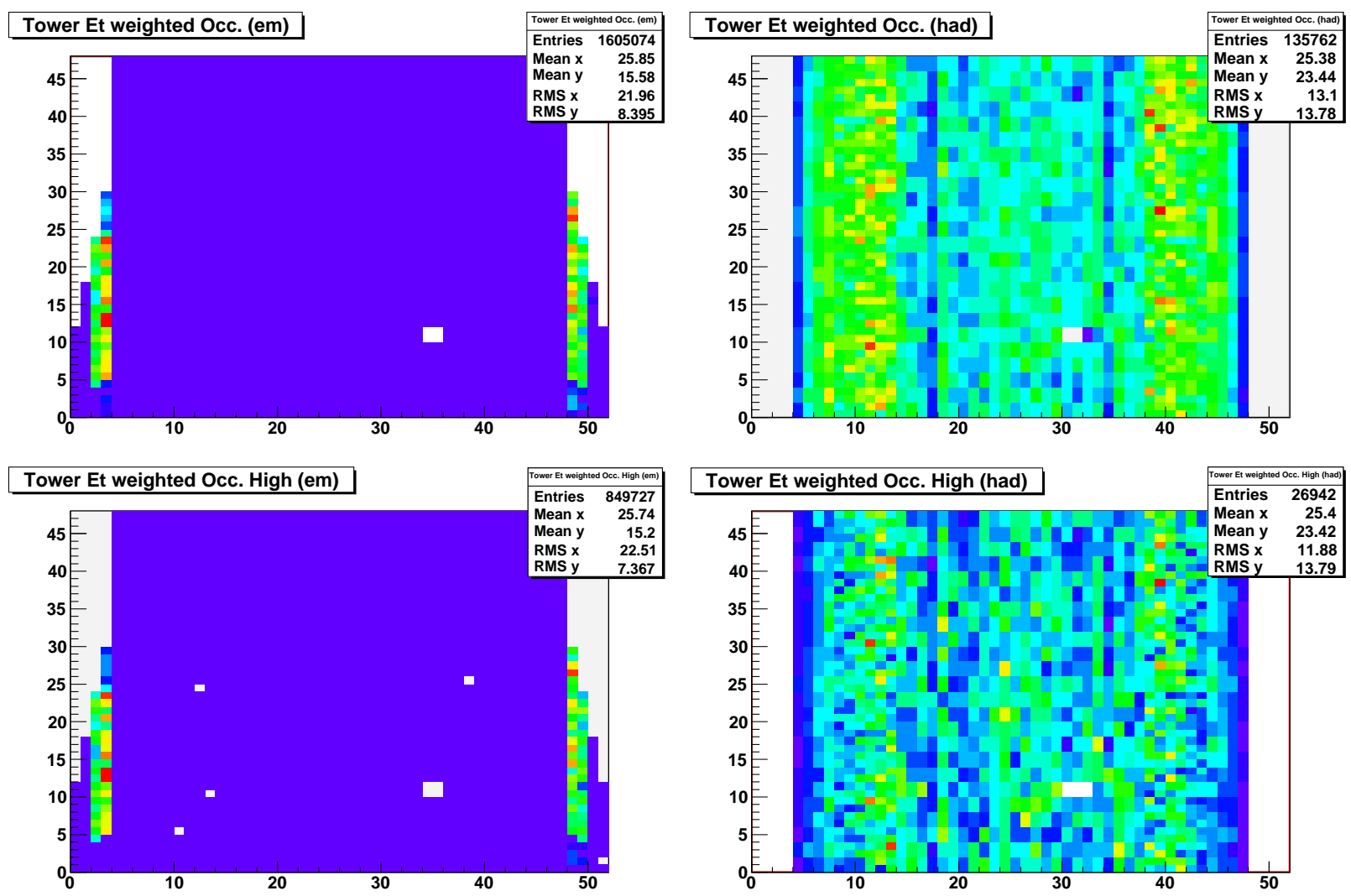

Fig. A.2: Occupancy of electromagnetic (left) and hadronic (right) towers for low (up) and high (down) energy thresholds for a particular run.

independent of $\phi$. From these occupancy distributions, with mean value $\mu$, one can expect to have an RMS ruled by two main contributions: a poissonian fluctuation $\sqrt{\mu}$ coming from the minimum bias occupancies and a gaussian effect coming from the calorimeter residual non-uniformities after the tower by tower relative calibrations are applied. These two effects can be parameterised as:

$$
\mathrm{RMS}=\sqrt{\mu+\alpha \mu^{2}}
$$

From this relation, the parameter $\alpha$ is determined to be $4 \%$ for the electromagnetic calorimeter and $3 \%$ for the hadronic calorimeter.

In general, the tagging is performed as follows: using the mean occupancy from each $\eta$ ring (removing the coldest and hottest towers to avoid biases), towers are tagged cold (hot) if they have an occupancy $6 \sigma$ away from the mean value $\mu$ minus (plus) $3 \alpha \mu$. This term $\alpha \mu$ accounts for the residual non uniformities uncertainties and a 3 sigma fluctuation is assumed. 


\section{A.2.3 Muons}

The muon system is more complex to monitor since it is divided in four subsystems: CMU, CMP, CMX and IMU. Different distributions are chosen to study the behaviour of each of them. One of the most powerful objects to monitor are the stubs, which are track segments in the muon chambers, since significant deviation from their expected number, when normalised by the corresponding luminosity, are a sign that the system may have been compromised. In addition, the average number of hits per stub, which control possible contributions from electronic noise, the deviations from the COT track extrapolation to the muon chamber, the deviations from expected muon energies distributions deposited in the electromagnetic and hadronic calorimeters and the di-muon invariant mass are extensively used for monitoring purposes. 


\section{Appendix B}

\section{Trigger Studies}

The $\mathbb{E}_{T}$ trigger efficiency was studied in order to place the $\mathbb{E}_{T}$ pre-selection cut well above the trigger efficiency turn-on curve. The $\mathbb{E}_{T}$ trigger that is being used (see Tab. 4.1) is a combined trigger in the sense that is requiring $\mathbb{E}_{T}>25 \mathrm{GeV}$ at Level 1 (L1) and two jets of $E_{T}>10 \mathrm{GeV}$ at Level 2 (L2). This trigger has been intensively studied by other analyses at CDF and here the discussion is limited to show that no effects from the trigger are present in the final distributions given the selection criteria employed.

\section{Level 1: $\mathbb{E}_{T}>25 \mathrm{GeV}($ L1_MET35)}

To estimate the trigger efficiency, a high $p_{T}$ muon sample, named bhmu0d, and a Jet20 data sample, named gjt10d, was used. The efficiency is defined as

$$
\varepsilon\left(\mathscr{E}_{T}\right)=\frac{N}{N_{T}}
$$

where $N$ is the number of events from the unbiased trigger that passed the L1_MET35 requirement and $N_{T}$ is the total number of events. Fig. B. 1 show the turn-on curves. A $50 \mathrm{GeV}$ value for the uncorrected offline $\mathbb{E}_{T}$ is already in the plateau. This translates into a corrected $\mathbb{E}_{T}$ value of 56 $\mathrm{GeV}$, well below the final cut that was applied.

\section{Level 2: Two jets of $E_{T}>10 \mathrm{GeV}\left(\mathrm{L2} \_\right.$MET35)}

To study the L2 trigger efficiency, the pre-scaled trigger (PSMET25) for $\mathbb{E}_{T}$ was used. This trigger path is only asking for $\mathbb{E}_{T}>25 \mathrm{GeV}$ at $\mathrm{L} 1$ and then it is pre-scaled at the other levels but no further requirement is implemented. Therefore,

$$
\varepsilon\left(E_{T}^{2 \mathrm{jet}}\right)=\frac{N}{N_{T}}
$$



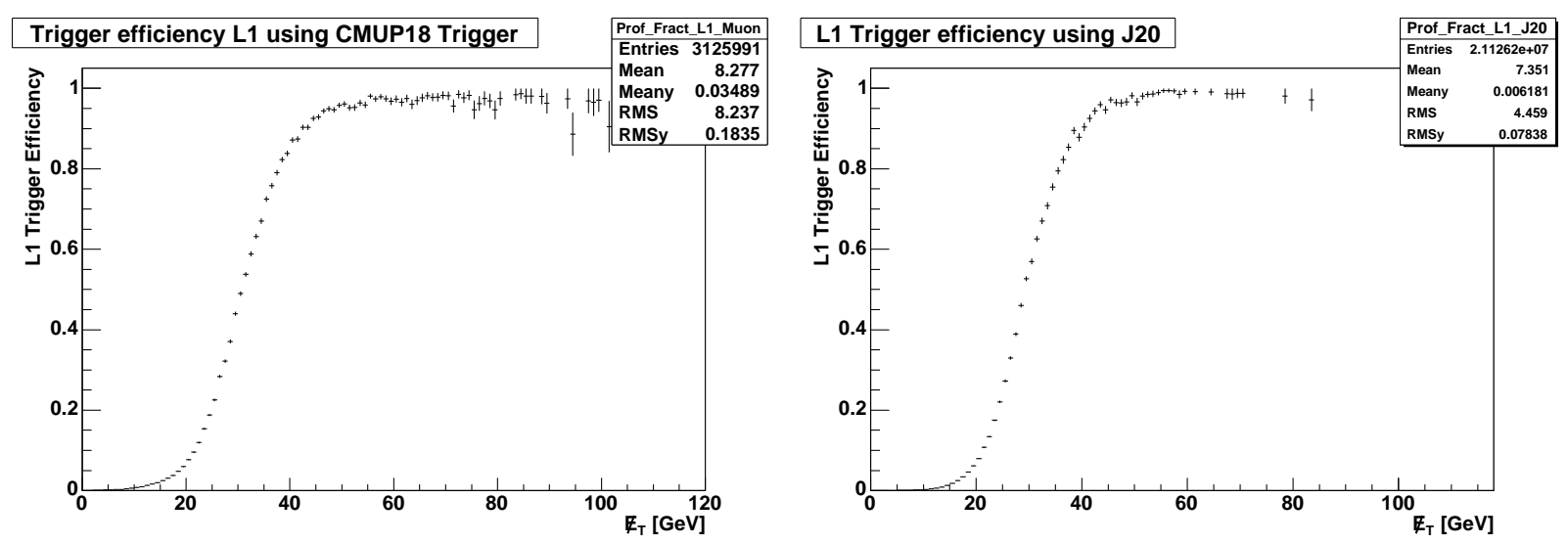

Fig. B.1: L1 trigger efficiency curve with respect to the offline $\not_{T}$ using high $p_{T}$ muon sample (left) and Jet20 (right).

where $N$ is the number of events from the PSMET25 trigger that passed the L2_MET35 requirement and $N_{T}$ is the total number of events that are present in the PSMET25 trigger path.

The turn-on curve for L2 with the $E_{T}$ of the second and the third jets can be seen in Fig. B.2. From the figures, the trigger achieves a $95 \%$ efficiency at $40 \mathrm{GeV}$ for the second jet and at $25 \mathrm{GeV}$ for the third. Tab. B. 1 compare events from MET35 and PSMET25 trigger after each pre-selection cut used in the analysis. As shown in this table, after all the pre-selection cuts the number of events passing both trigger paths is the same. This implies that no loss of efficiency is expected at the L2.
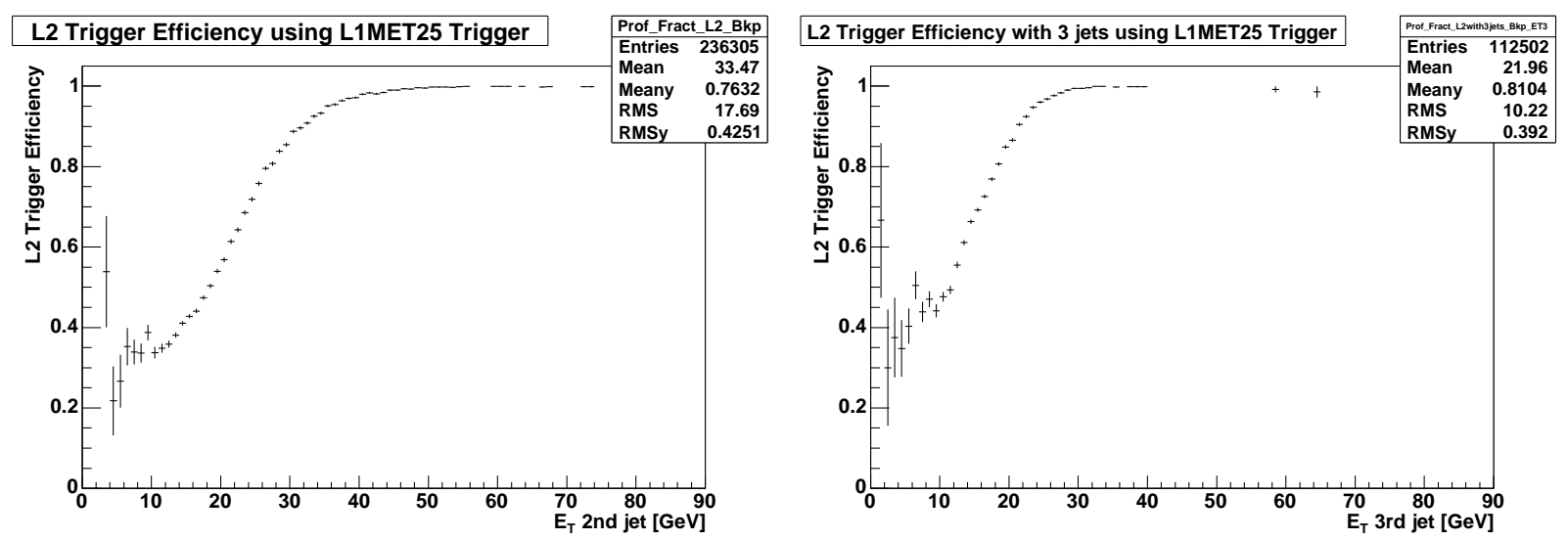

Fig. B.2: L2 trigger efficiency curve with respect to the transverse energy of the second jet (left) or the third jet (right). Both distributions are implemented using the pre-scaled missing transverse energy trigger. 


\begin{tabular}{|c|c|c|}
\hline \hline Cut Applied & L1 MET25 & + L2 TWO-JET10 \\
\hline+ Vertex Cut & 631723 & 249504 \\
\hline+3 jet Cut & 25409 & 25251 \\
\hline+ Central jet & 24561 & 24408 \\
\hline$+\mathscr{E}_{T}>70 \mathrm{GeV}$ & 117 & 116 \\
\hline All Pre-sel. Cuts & 99 & 99 \\
\hline \hline
\end{tabular}

Tab. B.1: The center column is the number events passing the L1-MET25 trigger path along with any cut shown on the left column. The right column shows the number of events in the center column including the L2 TWO-JET10 trigger requirement. The numbers shown are not prescaled.

\section{Level 3: $\mathbb{E}_{T}>35 \mathrm{GeV}(\mathbf{L 3}$ _MET35)}

To study the L3 trigger the high $p_{T}$ muon sample was used. The efficiency is defined as in Eq. (B.1) but now $N$ is the number of events from the CMUP18 trigger that passed the complete MET35 trigger path and $N_{T}$ is the number of events that passed the L1 and L2 requirements of the MET35 path. Fig. B.3 shows the turn-on curve with respect to the offline $\mathbb{E}_{T}$. This figure has been used to decide the minimum $\mathscr{E}_{T}$ cut to avoid trigger effects in the data used for this analysis. Being $50 \mathrm{GeV}$ the value at $95 \%$ efficiency, $60 \mathrm{GeV}$ was considered as the desirable threshold which takes into account the resolution of the energy correction. This value becomes $67 \mathrm{GeV}$ after applying the corrections to the $\mathbb{E}_{T}$. Offline, a cut on $70 \mathrm{GeV}$ was finally considered.

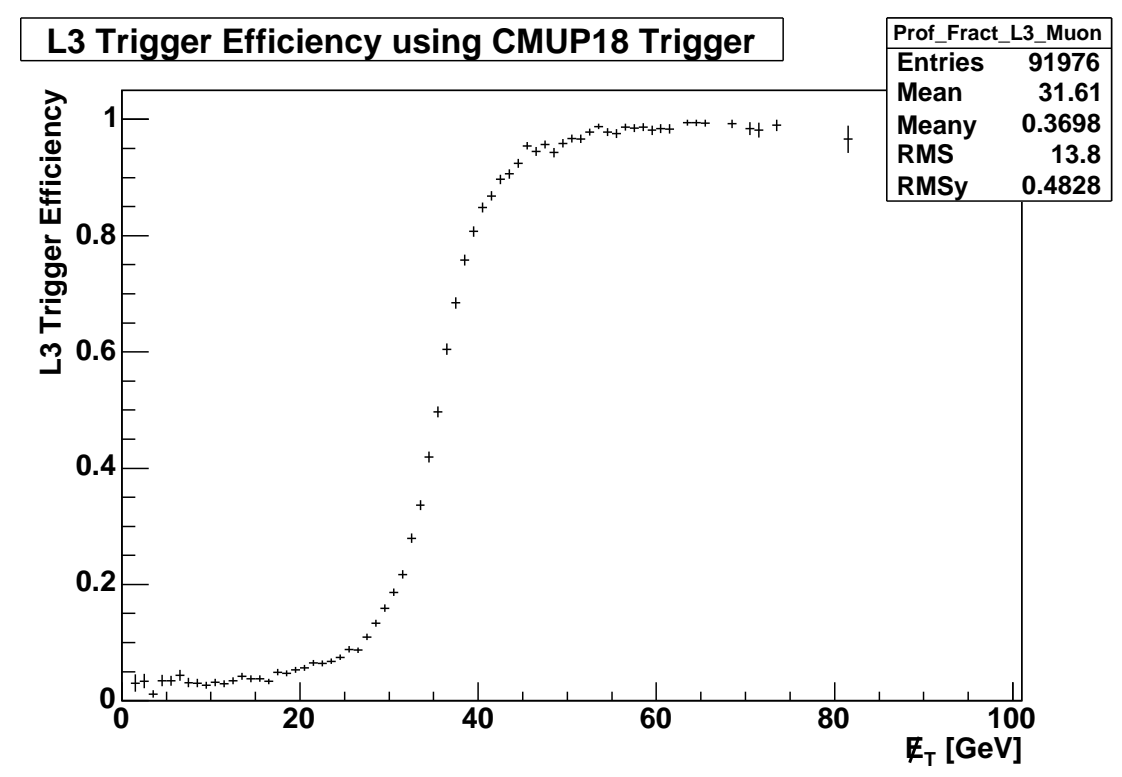

Fig. B.3: L3 trigger efficiency curve with respect to the offline $\not_{T}$ using high $p_{T}$ muon sample. 


\section{Appendix C}

\section{Beam Backgrounds and Cosmic Rays}

Beam backgrounds and cosmic rays are not directly related to the $p \bar{p}$ collision and can produce large $\mathbb{E}_{T}$ signatures when there is an overlapping with a $p \bar{p}$ collision. In addition, the presence of these kind of backgrounds is also responsible for the non-flat $\mathbb{E}_{T} \phi$ distribution, as it should be from the azimuthal symmetry of the experiment. As shown in Fig. C.1, the raw distribution shows a multiple-peak structure convoluted with a sinusoidal shape that will be explained below.

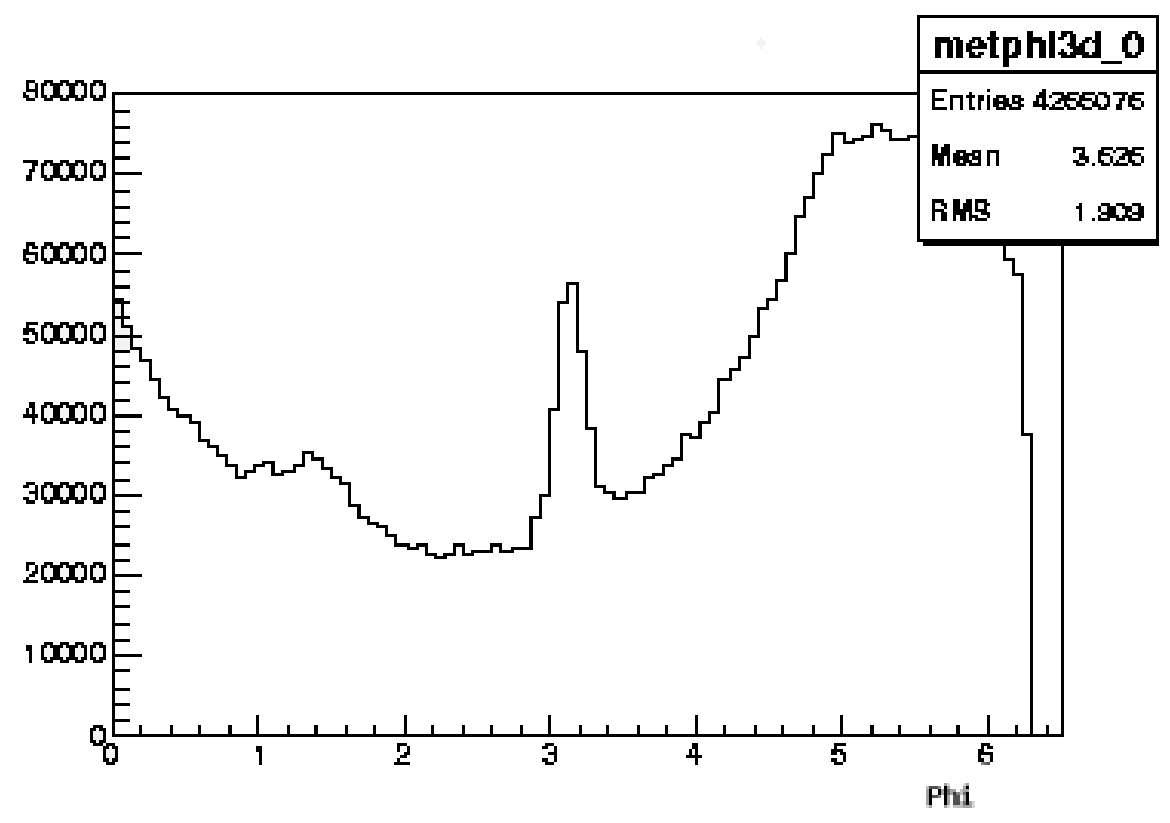

Fig. C.1: $\not_{T} \phi$ distribution without applying any cut. The peaks at different regions can be explained from the presence of other type of backgrounds not coming from the $p \bar{p}$ collision.

The beam halo background is constituted by a number of particles (usually muons) that have been deviated from the main trajectory. When these particles, which travel parallel to the beam 
line, traverse the detector at the region of the calorimeter, as shown in Fig. C.2, they begin to shower and can deposit significant amount of energy. As shown in Fig. C.3 these halo muons usually pass through the central calorimeter at an azimuthal angle $\phi \sim 0$, the plane of the accelerator. Thus, in the $\phi$ distribution of the $\mathbb{E}_{T}$ a peak at $\phi \sim \pi$ is observed.

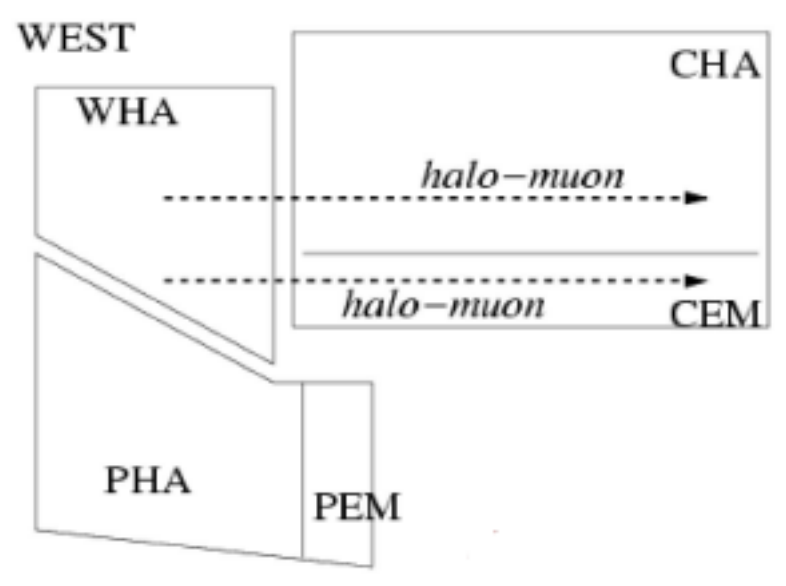

EAST

Fig. C.2: Beam halo particles trajectory through the CDF calorimetry.

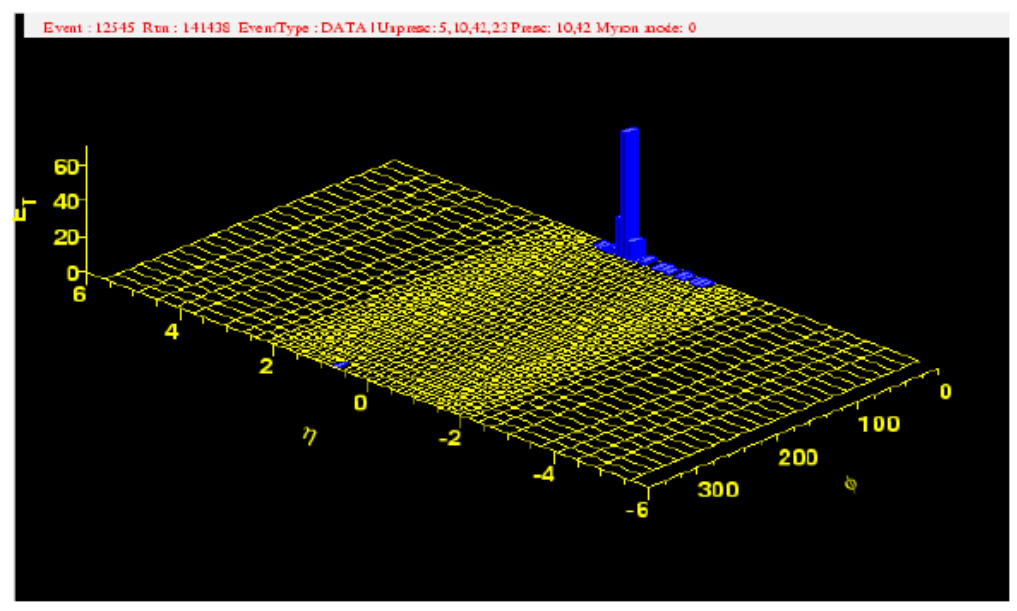

Fig. C.3: Example of beam halo energy deposition.

On the other hand, losses from the proton and anti-proton beams occuring near the detector, typically populate the plug calorimetric towers closest to the beam line. In Fig. C.4 the average transverse energy deposited in these towers is presented. As it is shown, the energy depositions tend to be aligned along the $y$ axis. The reason is that there exist a $\sim 4 \mathrm{~cm}$ gap between the two halves of the intermediate shielding walls. This gap was only partially covered and it still allows 
some of the losses to pass through. This kind of background explains the two peaks around $\pi / 2$ and $3 \pi / 2$ in Fig. C. 1 .

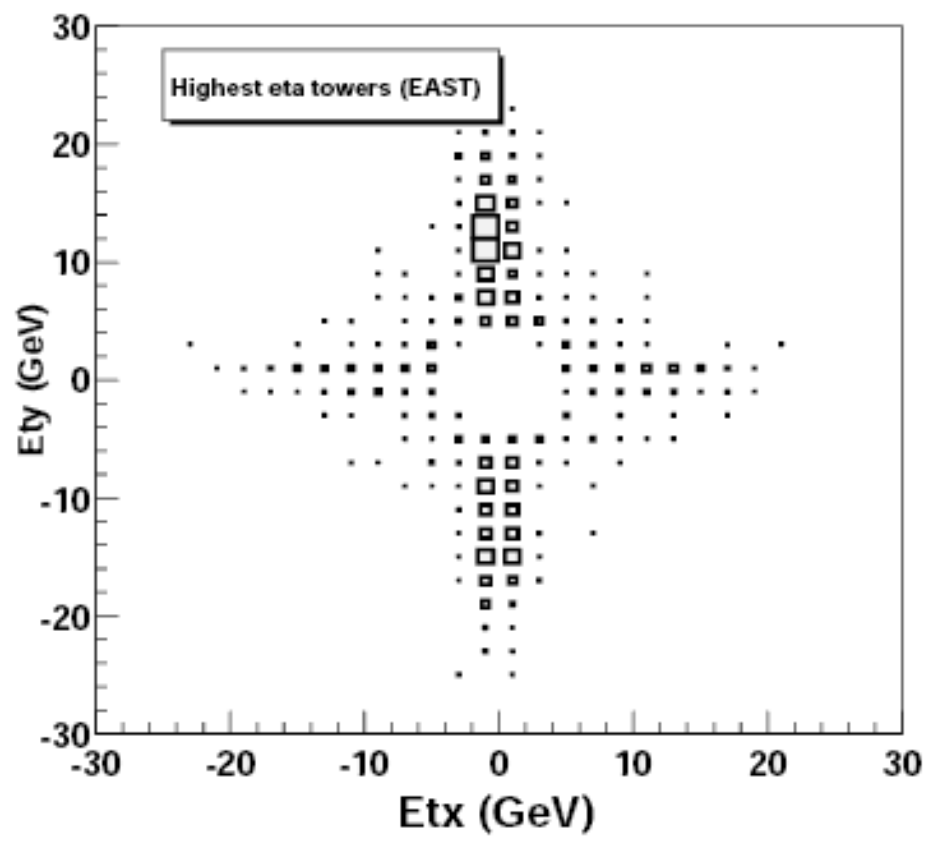

Fig. C.4: $\mathrm{X}$ and $\mathrm{Y}$ components of the transverse energy deposited in the closest towers to the beam line for the east plug.

Finally, cosmic muons can traverse the calorimeter and deposit a significant amount of energy in one side of the central calorimeter, resulting in a large imbalance of the measured energy.

\section{C.1 Cuts to remove beam-related backgrounds}

Different type of cuts were proposed to remove these backgrounds:

- A missing transverse energy cut $\left(\mathbb{E}_{T}>45 \mathrm{GeV}\right)$.

- At least one vertex $\left(V_{Z}<60 \mathrm{~cm}\right)$.

- At least 2 jets $\left(\mathbb{E}_{T}>10 \mathrm{GeV}\right)^{1}$.

These cuts are softer than the ones applied in the analysis (see Section 4.1.2) but the purpose here was to study their effects on the $\mathbb{E}_{T} \phi$ distribution. The effect of the cuts is shown in Fig. C.5

\footnotetext{
${ }^{1}$ Here, the energies of the jets are not corrected for the detector effects and multiple interactions contributions.
} 
where the $\mathbb{E}_{T}$ cut helps removing the background from beam losses but leaves the peak due to the beam halo background untouched. In contrast, by requiring two jets and one vertex within $60 \mathrm{~cm}$ of the collision point, the beam halo background is clearly reduced.
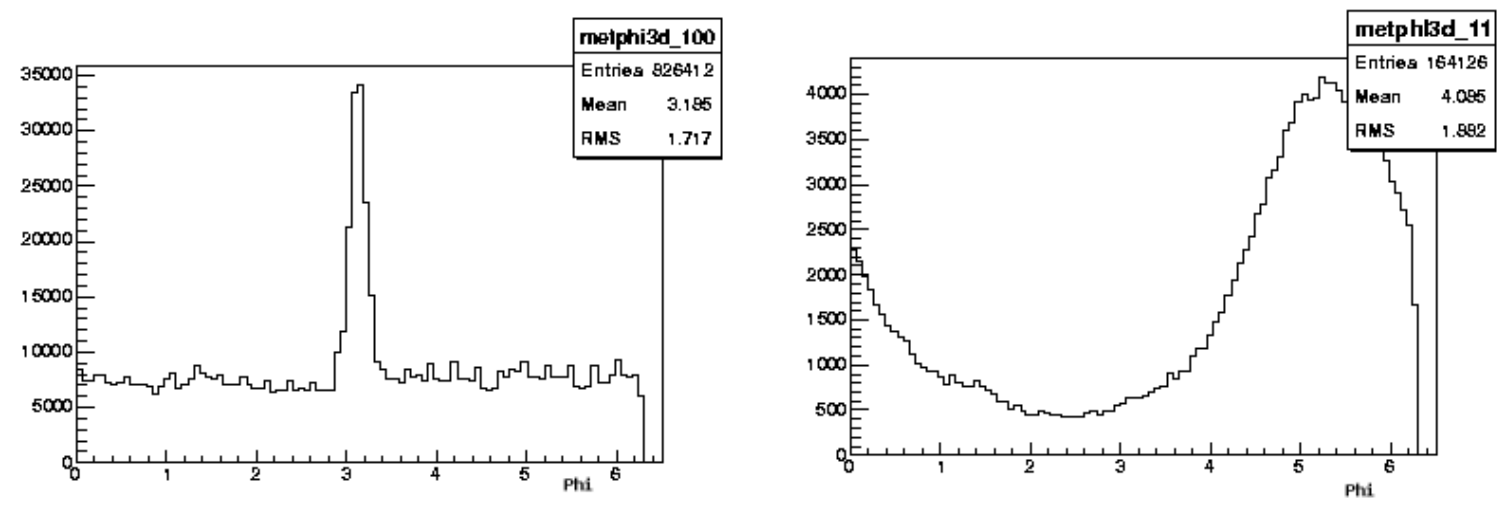

Fig. C.5: $\not_{T}$ spectrum in $\phi$ of a subset of the data after applying the $\not_{T}>45 \mathrm{GeV}$ (left) or the vertex and two jets requirements (right).

Fig. C.6 shows the effect of the combination of both cuts. As expected, the statistics of the sample is much reduced but the distribution is essentially flat.

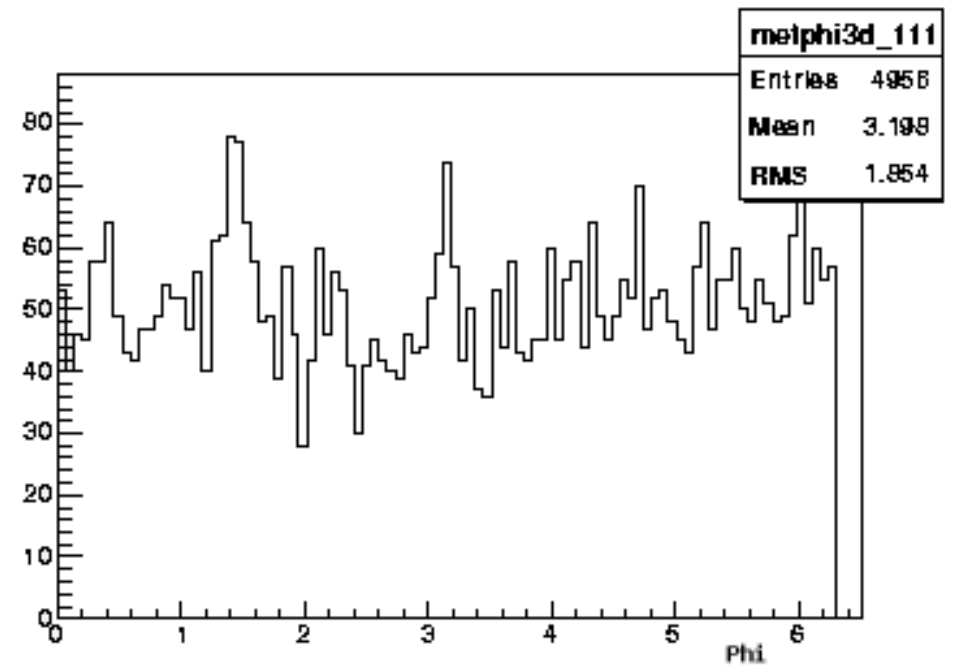

Fig. C.6: $\not_{T}$ spectrum in $\phi$ of a subset of the data after applying the $\not_{T}>45 \mathrm{GeV}$ cut and the requirements of one vertex $\left(V_{Z}<60 \mathrm{~cm}\right)$ and at least two jets.

\section{C.1.1 ECHF and EEMF variables}

In addition to the previous cuts, some specific variables were introduced to reduce the contribution from cosmic rays and the beam-related backgrounds in the case where they are overlapped with a 
real hard scattering collision. Two variables were used for these purposes: EEMF and ECHF.

\section{EEMF}

This variable is defined as:

$$
\mathrm{EEMF}=\frac{\sum_{\mathrm{jets}} E_{T}^{\mathrm{jet}} \cdot f_{\mathrm{emf}}^{\mathrm{jet}}}{\sum_{\mathrm{jets}} E_{T}^{\mathrm{jet}}}
$$

where $f_{\mathrm{emf}}^{\text {jet }}$ is the fraction of the transverse electromagnetic energy of the jet, and the sum is performed over the three leading jets of the event.

It is expected that particles coming from the beam halo will deposit energy mainly in the hadronic calorimeter giving an EEMF close to zero. This situation can be seen in Fig. C.7 where this quantity is shown for data and MC. The major part of this background is removed by introducing a cut on EEMF $>0.15$.

\section{EEMF after basic cuts (except EEMF and ECHF)}

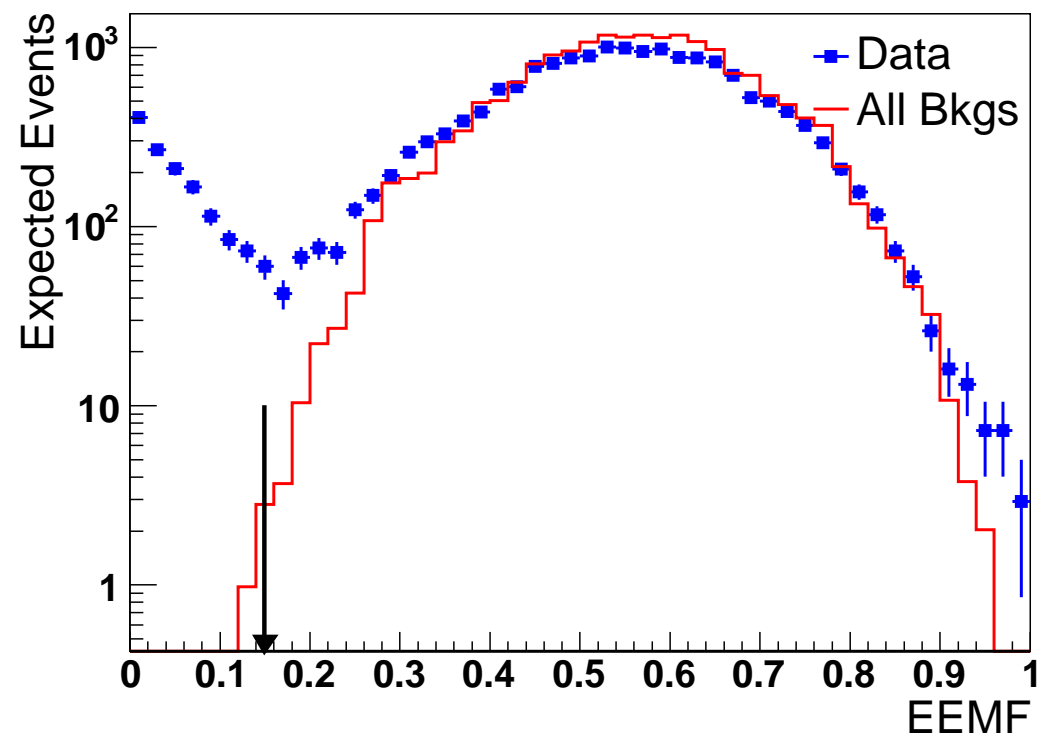

Fig. C.7: EEMF for data and all the SM backgrounds (MC simulated). The plot includes the pre-selection cuts except the cuts on EEMF and ECHF.

\section{ECHF}

Another characteristic of the beam background events is that tracks do not point to the region where the particle has deposited energy in the calorimeter. The quantity ECHF is defined for 
central jets $\left(\left|\eta^{\text {jets }}\right|<1.1\right)$ as the averaged ratio between the momentum of the jet, as computed using tracks, and the jet transverse energy:

$$
\mathrm{ECHF}=\frac{1}{N_{\text {jets }}} \sum_{\text {jets }} \frac{\sum_{\text {tracks }} P_{T}^{\text {track }}}{E_{T}^{\text {jet }}},
$$

where only tracks in a cone of radius 0.4 around the jet's direction are considered. These tracks are required to pass the quality cuts described in Section 4.1.2.

Fig. C.8 shows the ECHF distribution for data and MC. The shift seen in the data distribution with respect to the Monte Carlo is due to beam backgrounds in the sample. A cut on ECHF $>0.15$ is implemented to remove these backgrounds.

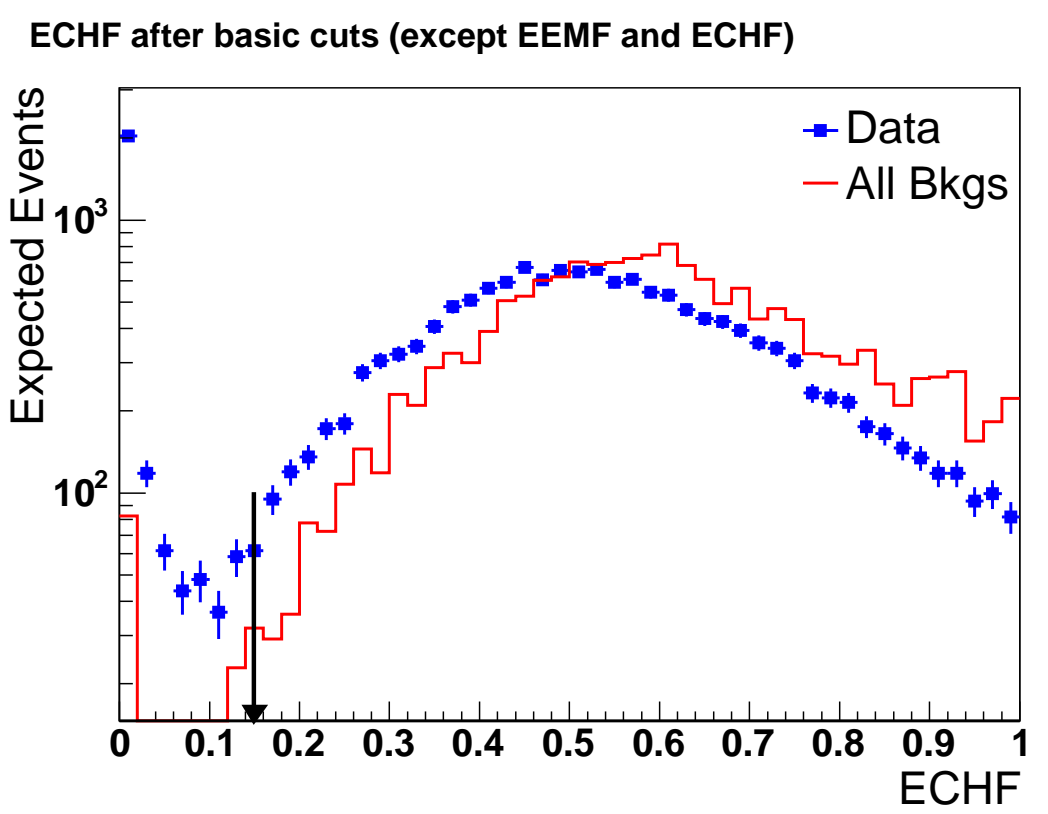

Fig. C.8: ECHF for data and MC. The plot includes the pre-selection cuts except the cuts for EEMF and ECHF.

\section{C.2 Study of the beam losses in the plug}

A dedicated study to understand the origin of the sinusoidal modulation observed in the $\mathbb{E}_{T}$ distribution in $\phi$ was performed. This study only used a small subgroup of data $\sim 4 \mathrm{pb}^{-1}$ and consisted in observing the effect of removing the highest $\eta$ towers from the $\mathbb{E}_{T}$ calculation. Fig. C.9 show the $\mathscr{E}_{T}$ distribution in $\phi$ considering the whole calorimeter (left), only considering towers with $|\eta|<1.93$ (middle) and without considering the plugs $(|\eta|<1.1)$ (right). Appart from the central peak in the distributions, which corresponds to beam halo energy depositions, the sinusoidal behaviour of the $\mathbb{E}_{T}$ is becoming flatter when removing the towers of both (east and west) plugs. 
Since this behaviour was not reproduced when removing exclusively eastern or western towers, each plug was divided in sections of 60 degrees in $\phi$ starting from $\phi=0$. This segmentation allowed the study of the energy distribution in $\phi$ for each of the plugs. Fig. C.10 show the mean transverse energy deposited in the east and west electromagnetic and hadronic plugs. It is noticeable the similar behaviour of the distributions. Since the mean energy is approximately higher for similar regions of both east and west plugs, the conclusion was that the beam was slightly out of the center. This explains the mentioned effect of the beam losses in the plugs and why removing exclusively the east or the west plugs did not remove completely the oscillation in $\phi$.
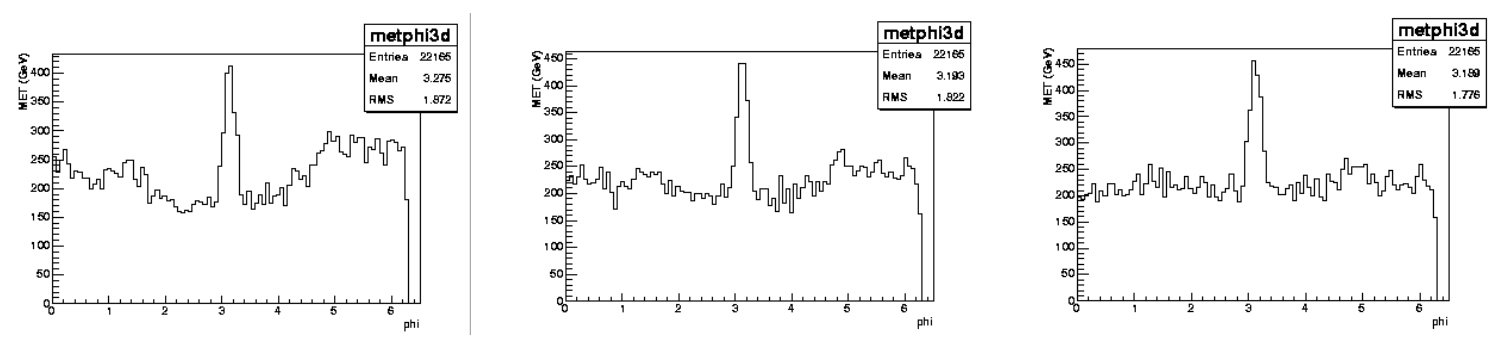

Fig. C.9: $\not_{T}$ spectrum in $\phi$, with the characteristic peaks already presented, with the whole calorimeter (left), with only considering $|\eta|<1.93$ towers (middle) and without the plugs $(|\eta|<1.1)$ (right).
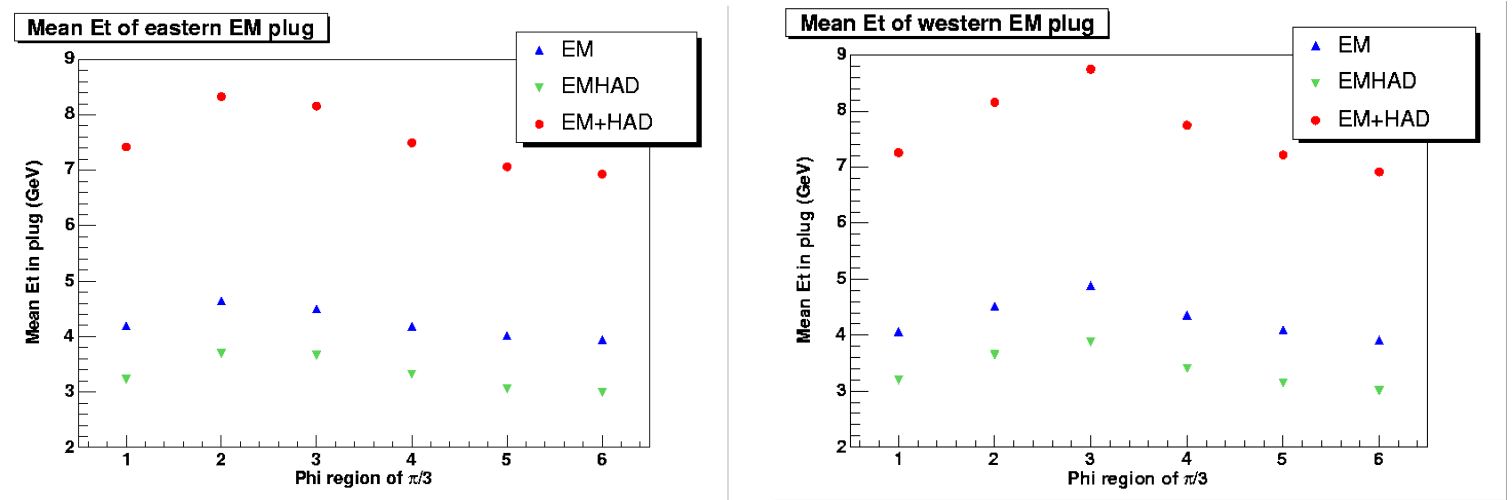

Fig. C.10: Mean transverse energy deposited in the east (left) or west (right) plugs in different $\phi$ regions. The $\phi$ regions cover $60^{\circ}$ and are numbered from 1 to 6 , starting from $\phi=0$. 


\section{Appendix D}

\section{Track Validation}

A dedicated study to validate the track definition given in Section 4.1.2 was performed using the inclusive high $\mathrm{p}_{\mathrm{T}}$ muon sample (bhmu0d). The integrated luminosity for the sample is $236 \mathrm{pb}^{-1}$, and the trigger path requires a CMUP track of $18 \mathrm{GeV} / \mathrm{c}$.

The $\mathrm{Z}$ mass peak was reconstructed using the two highest isolated tracks in the event. To ensure that all the events correspond to $Z \rightarrow \mu \mu$ a tight mass constraint of $86<\mathrm{M}_{\text {iso }}<96 \mathrm{GeV} / \mathrm{c}^{2}$ was applied. In addition, at least one central track with $|\eta|<0.5$ was required and a cosmic ray cut implemented. Fig. D.1 shows the reconstructed mass for data and MC samples. The broader data distribution is understood since not all the corrections have been applied. Nonetheless, the normalisation is fairly good: data account for 24 events and Monte Carlo for 28 events in this region. This was considered enough to ensure a good definition for tracks in the selection criteria. As a final check, the number of isolated tracks in the data sample used in the analysis was compared with the one predicted by the MC just after the pre-selection cuts. The agreement is shown in Fig. D.2. 


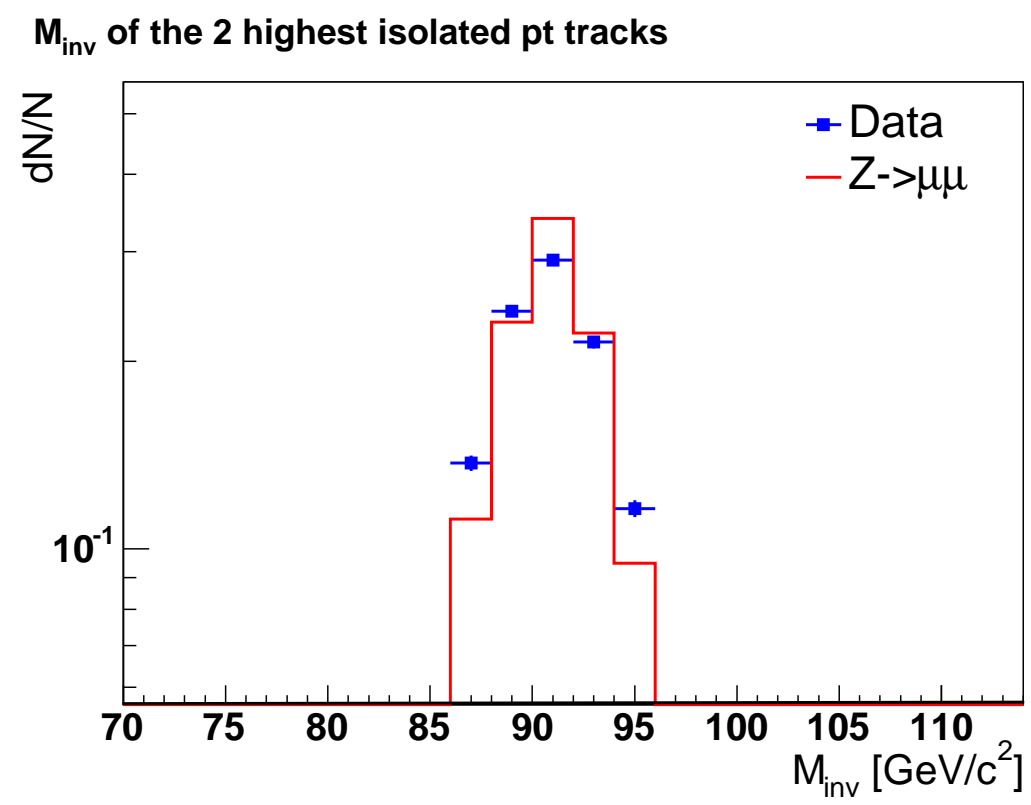

Fig. D.1: Invariant mass of the two highest isolated tracks for bhmu0d data and $Z \rightarrow \mu \mu$ Monte Carlo.

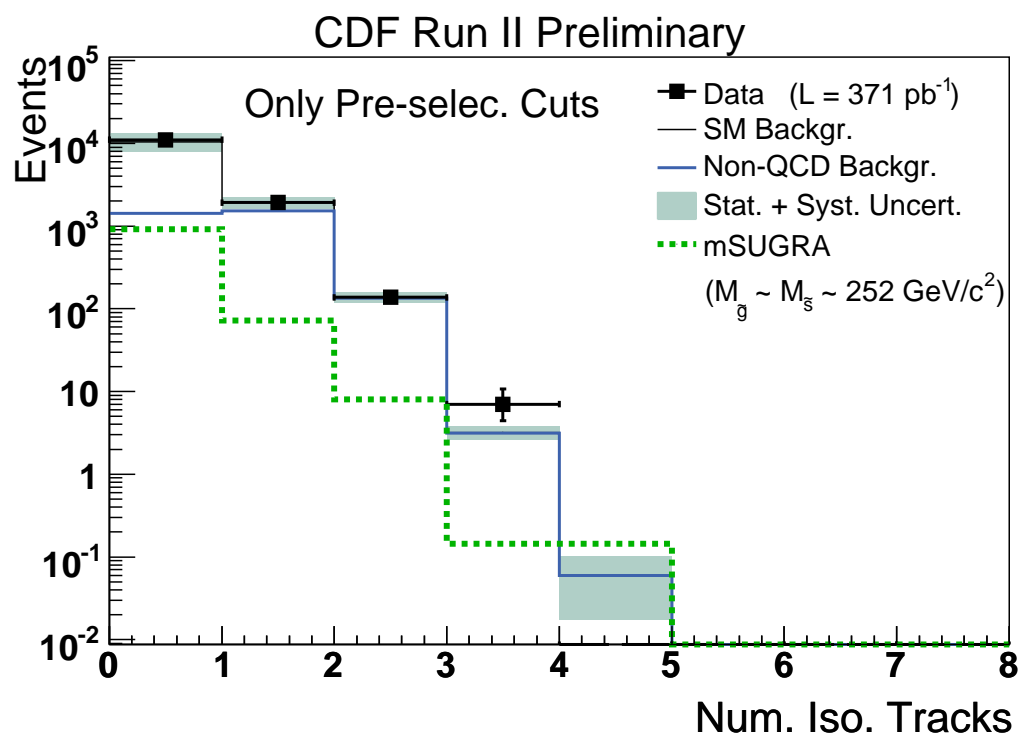

Fig. D.2: Number of isolated tracks just after the pre-selection cuts using the emet0d data sample. 


\section{Appendix E}

\section{PYTHIA vs ISAJET}

The predicted squark and gluino masses by ISAJET and PYTHIA agree well with differences smaller than $1 \%$, see Fig. E.1. The observed differences are negligible for this study and can be attributed to the different numerical methods employed in solving the renormalisation group equations that determine the running masses.
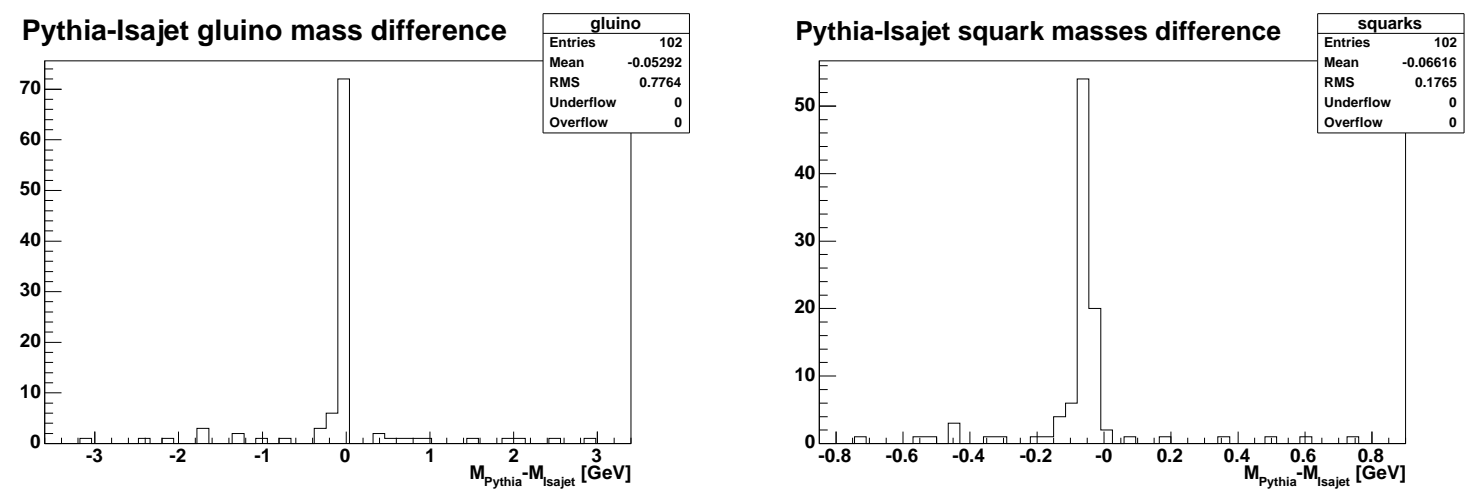

Fig. E.1: Mass differences between PYTHIA and ISAJET for gluino (left) and squarks (right)

The comparison between ISAJET and PYTHIA hadronic final states (see Fig. E.2) indicates that, for fixed squark and gluino masses, ISAJET produces much harder distributions than PYTHIA, which translates into a significantly larger signal selection efficiencies and better exclusion limits when ISAJET is used instead of PYTHIA. This is attributed to the limited (non-proper) initial- and final-state gluon radiation in ISAJET, compared to that implemented in PYTHIA, which makes ISAJET not suitable for this analysis. 

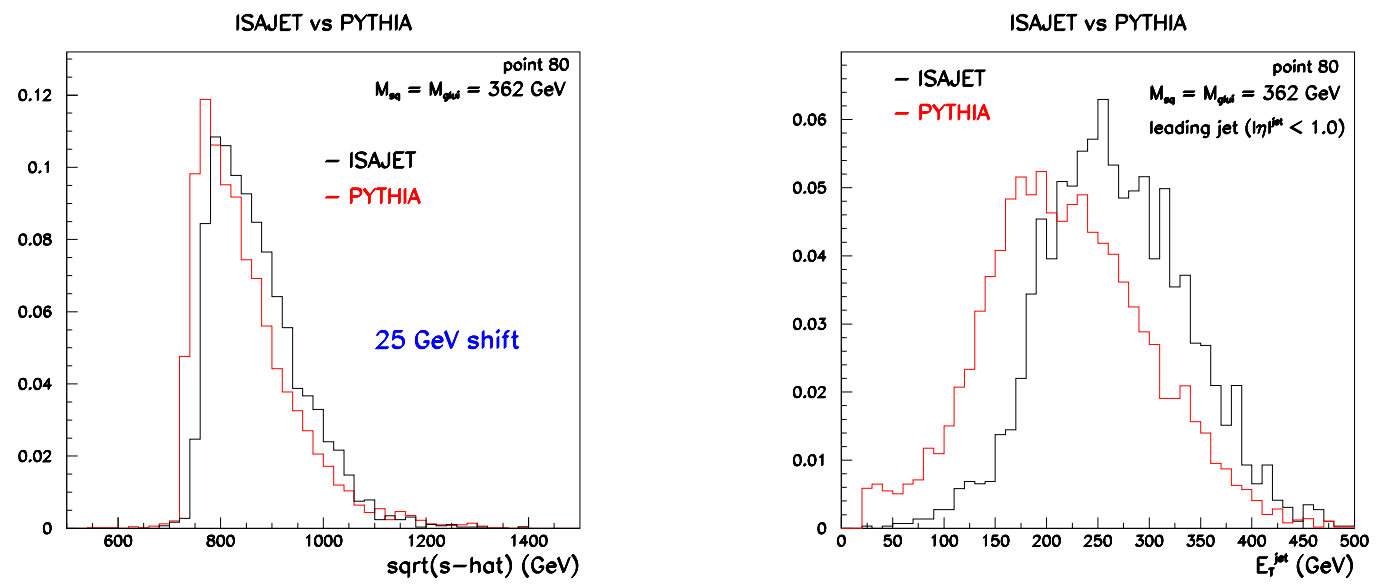

Fig. E.2: Difference on $\hat{s}$ between ISAJET and PYTHIA (left). Difference on the transverse energy of the leading jet at the hadron level (right). 


\section{Appendix F}

\section{QCD Studies}

\section{QCD Normalisation Study}

The cross section for QCD processes can be eight orders of magnitude above the signal cross section. These processes are very sensitive to the presence of partially instrumented regions in the calorimeter and differences in the jet energies which can lead to transverse energy imbalances.

QCD is generated using PYTHIA MC with the Tune A parameterisation. A dedicated measurement of the QCD multijet cross section was performed with the aim to extract the QCD MC normalisation directly from the data. This study used the Jet 20 trigger, which requires a jet with a single tower of $\mathrm{E}_{\mathrm{T}}>5 \mathrm{GeV}$ at Level $1(\mathrm{~L} 1)$; a jet with $\mathrm{E}_{\mathrm{T}}>15 \mathrm{GeV}$ with the $\mathrm{L} 2$ jet reconstruction algorithms; and finally, a jet with $\mathrm{E}_{\mathrm{T}}>20 \mathrm{GeV}$ at L3. Due to the large cross sections of the processes the trigger is prescaled at $\mathrm{L} 1$ and $\mathrm{L} 2$, meaning that only part of the data that follows the correspondent requirements is stored. The trigger has a turn-on which reach a plateau at corrected jet energies of around $30 \mathrm{GeV}$. The measurement was compared to PYTHIA MC predictions.

In order to avoid biases from potential signal regions, the following selection criteria were applied to select multijet events in a region with moderate $\mathbb{E}_{T}$ and total transverse energy:

- $\left|V_{Z}\right|<60 \mathrm{~cm}$

- At least three jets with $E_{T}^{\text {jet }}>25 \mathrm{GeV}$ and $\left|\eta^{\text {jet }}\right|<2.0$

- $E_{T}^{\mathrm{jet} 1}>90 \mathrm{GeV}$ and $E_{T}^{\mathrm{jet} 2}>60 \mathrm{GeV}$.

- At least one central jet.

- $\mathbb{E}_{T} / \sqrt{E_{T}}<3.5 \mathrm{GeV}^{-1 / 2}$

- $\mathscr{E}_{T}<70 \mathrm{GeV}$ and $E_{T}^{\mathrm{jet} 1}+E_{T}^{\mathrm{jet} 2}+\mathbb{E}_{T}<100 \mathrm{GeV}$. 
These requirements are intended to select QCD events with no significant $\mathbb{E}_{T}$ and with low $H_{T}$ in order not to bias the normalisation in the region where the signal is expected.

Fig. F.1 to Fig. F.5 show comparisons between the data and the PYTHIA predictions for the jet multiplicity, the transverse energies of the three leading jets and the $\mathbb{E}_{T}$. Fig. F.6 shows the ratio between data and $\mathrm{MC}$ for the $\mathbb{E}_{T}$ distribution. A $k$-factor of the order of 1.0 was obtained. The study was repeated in four separate region in $\mathbb{E}_{T}$ and $E_{T}^{\mathrm{jet} 1}+E_{T}^{\mathrm{jet} 2}+\mathbb{E}_{T}$ to test the validity of the measured $k$-factor for different event tolopogies (see Fig. F.7 to Fig. F.11). There is no indication that a different $k$-factor was necessary in the different regions.

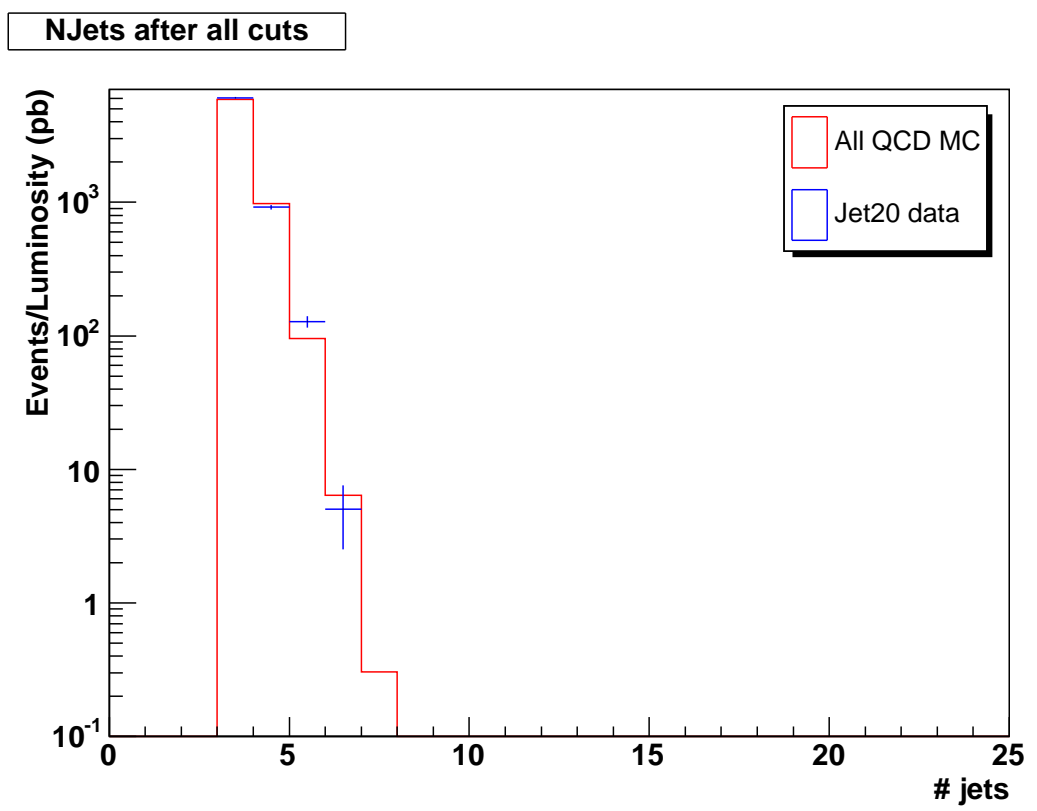

Fig. F.1: Distribution of number of jets in the QCD control region. 


\section{$E_{T}$ of leading jet after all cuts}

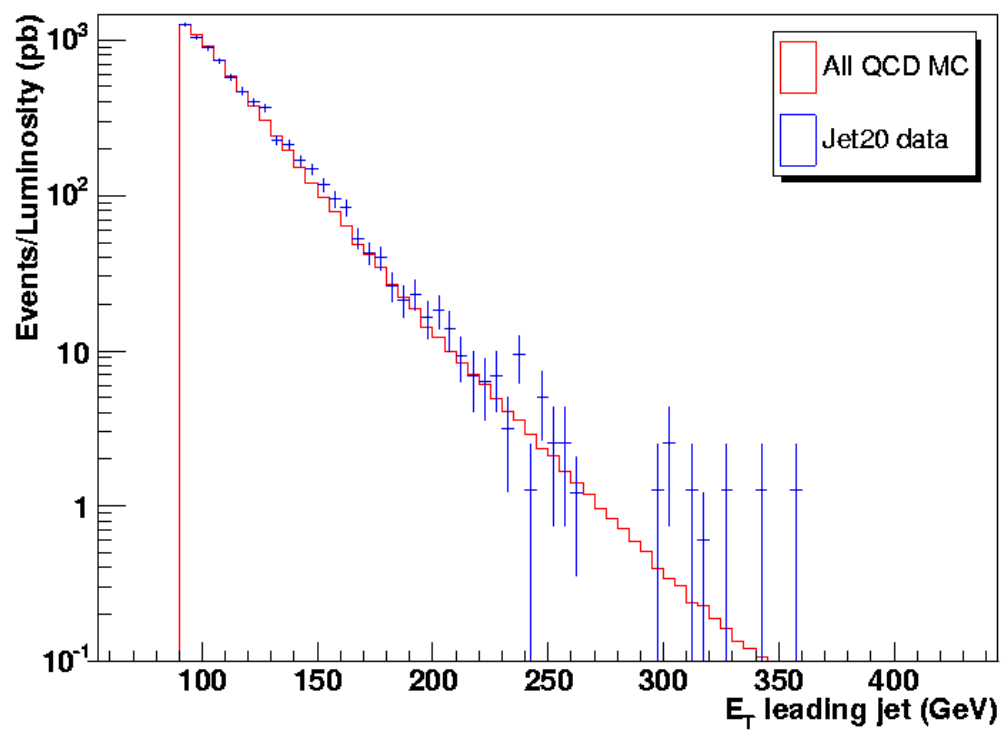

Fig. F.2: Distribution of the $E_{T}^{\text {jet1 }}$ inside the QCD control region.

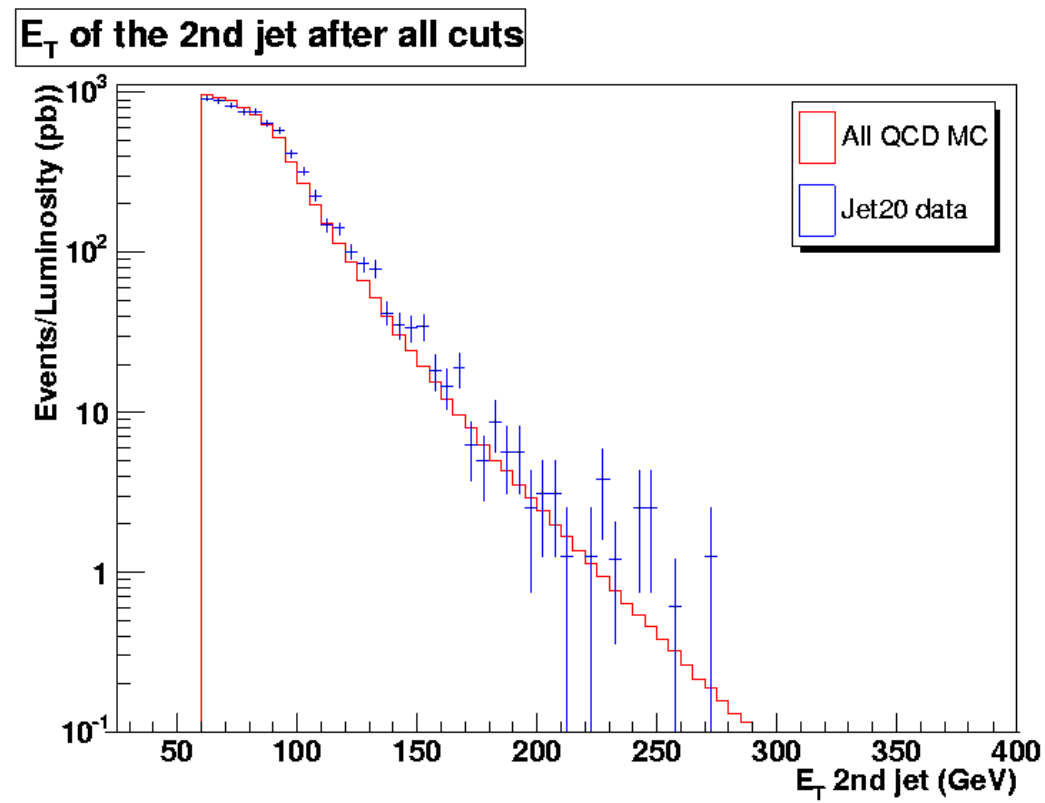

Fig. F.3: Distribution of the $E_{T}^{\text {jet2 }}$ inside the QCD control region. 


\section{$E_{T}$ of the 3rd jet after all cuts}

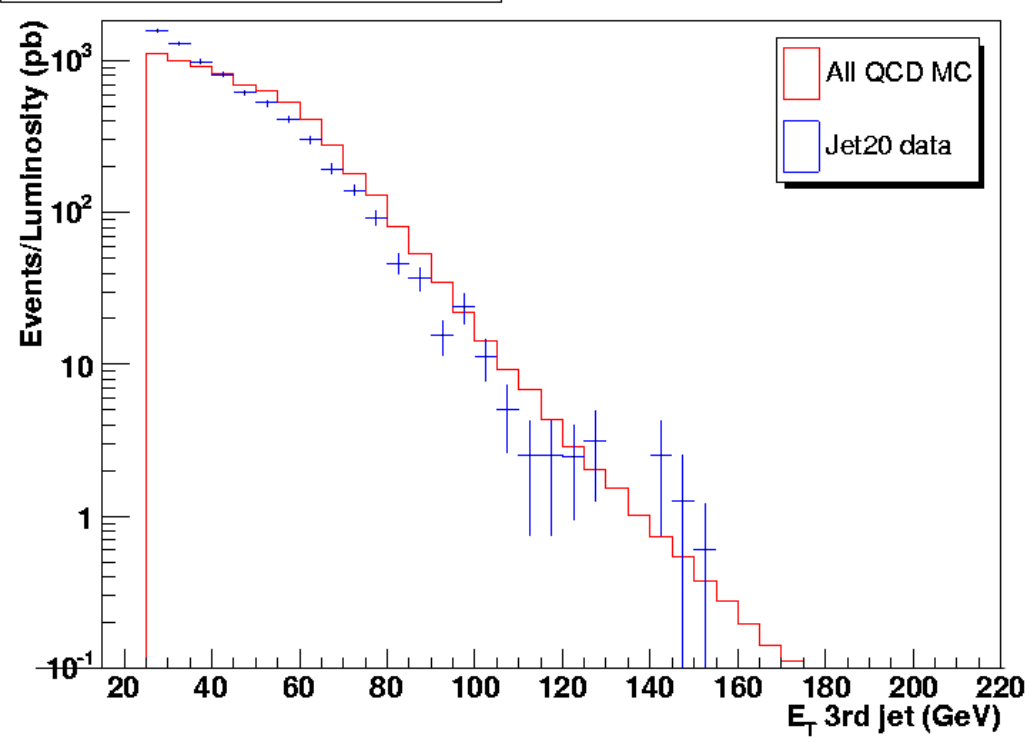

Fig. F.4: Distribution of the $E_{T}^{\text {jet3 }}$ inside the QCD control region.

\section{MET after all cuts}

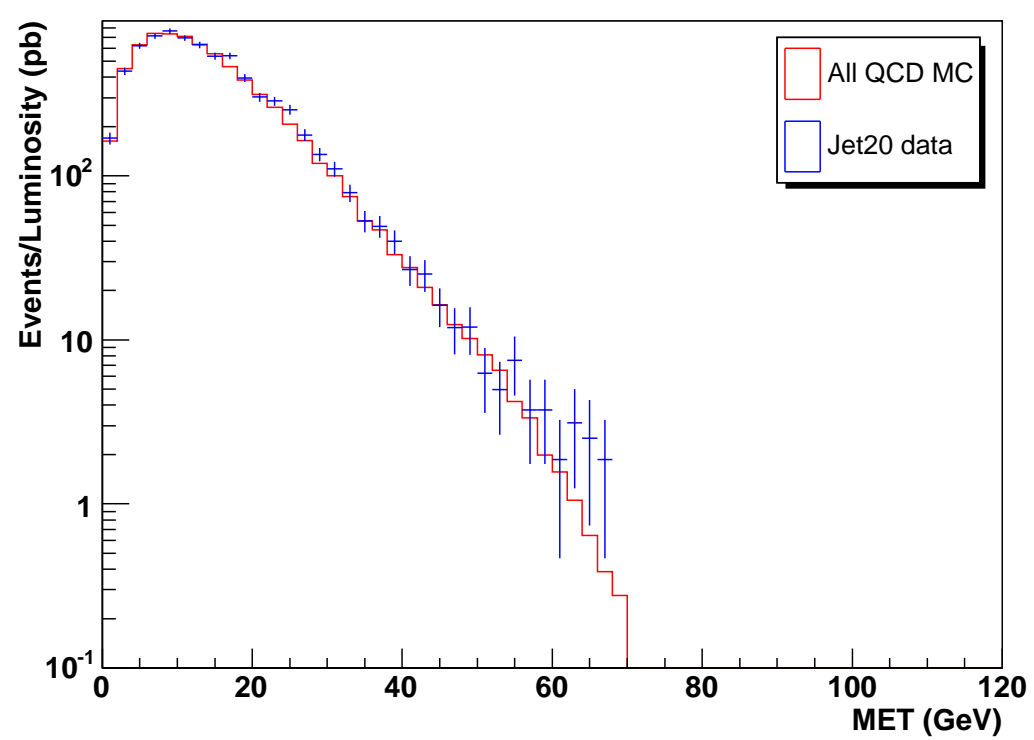

Fig. F.5: Distribution of $\not_{T}$ inside the QCD control region. 
Data/MC for MET

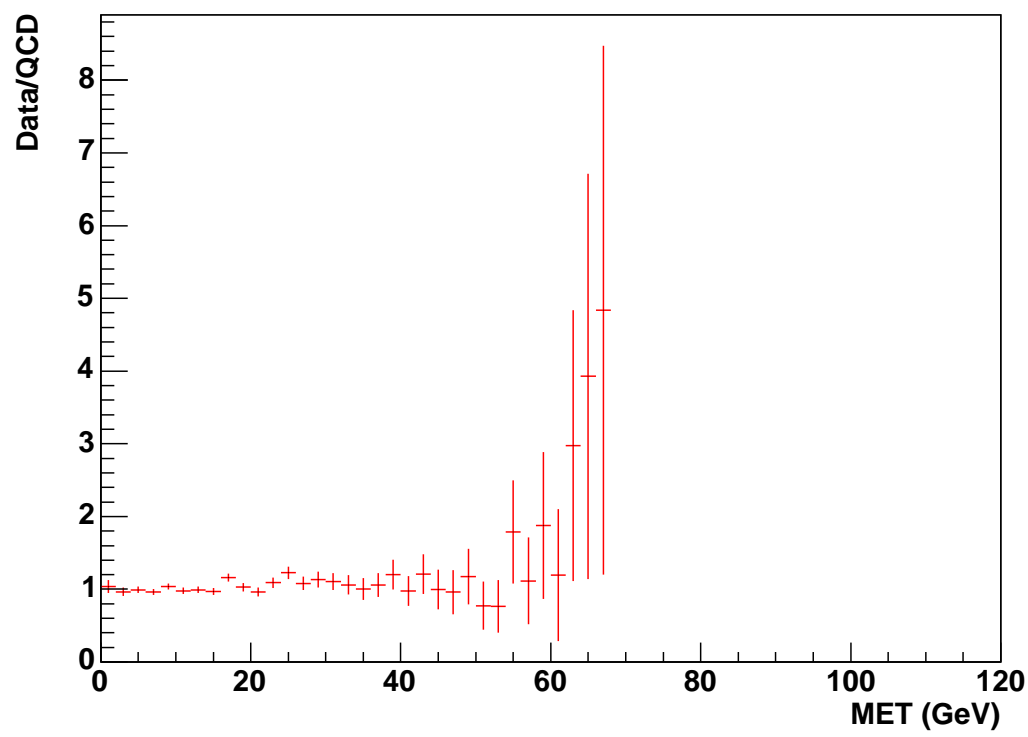

Fig. F.6: Ratio between data and $\mathrm{MC}$ for the $\not_{T}$ distribution inside the QCD control region.

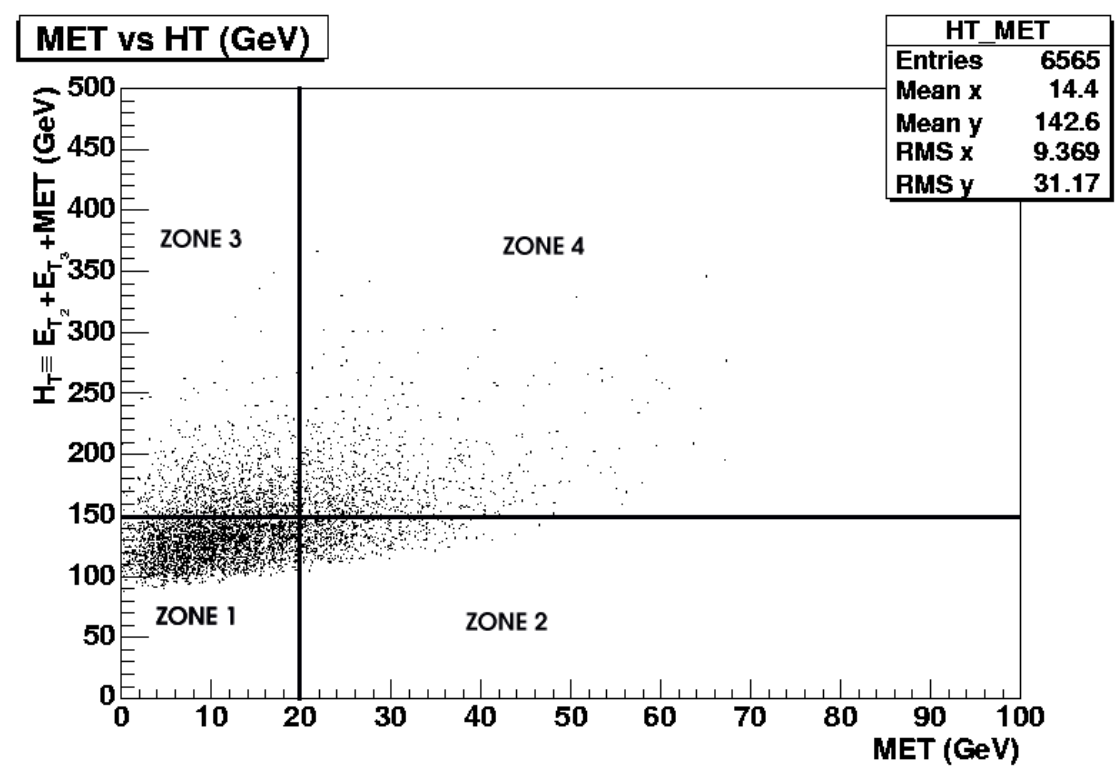

Fig. F.7: Definition of zones in $\not_{T}$ vs $H_{T}$ plane inside the QCD control region. 


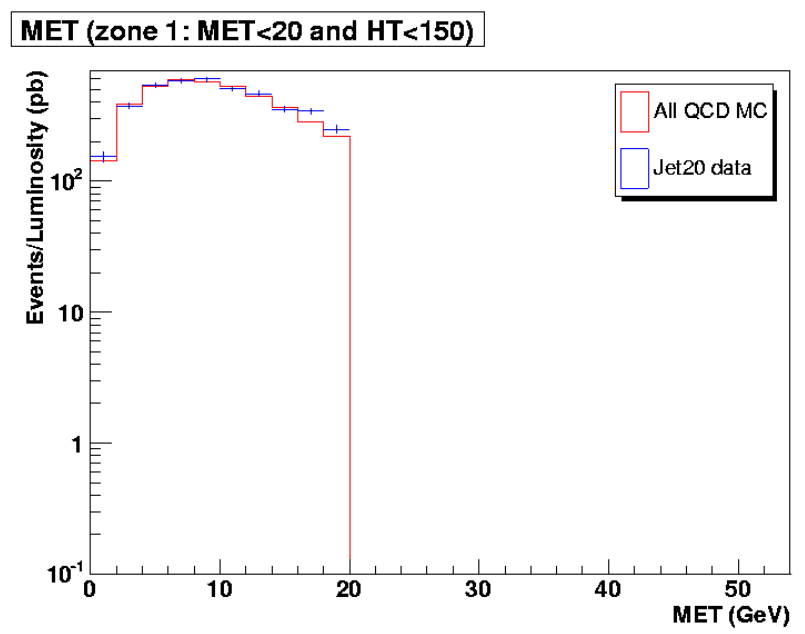

Fig. F.8: Distribution of $E_{T}$ inside the QCD control region (zone 1).

\section{MET (zone 2: MET>=20 and HT<150)}

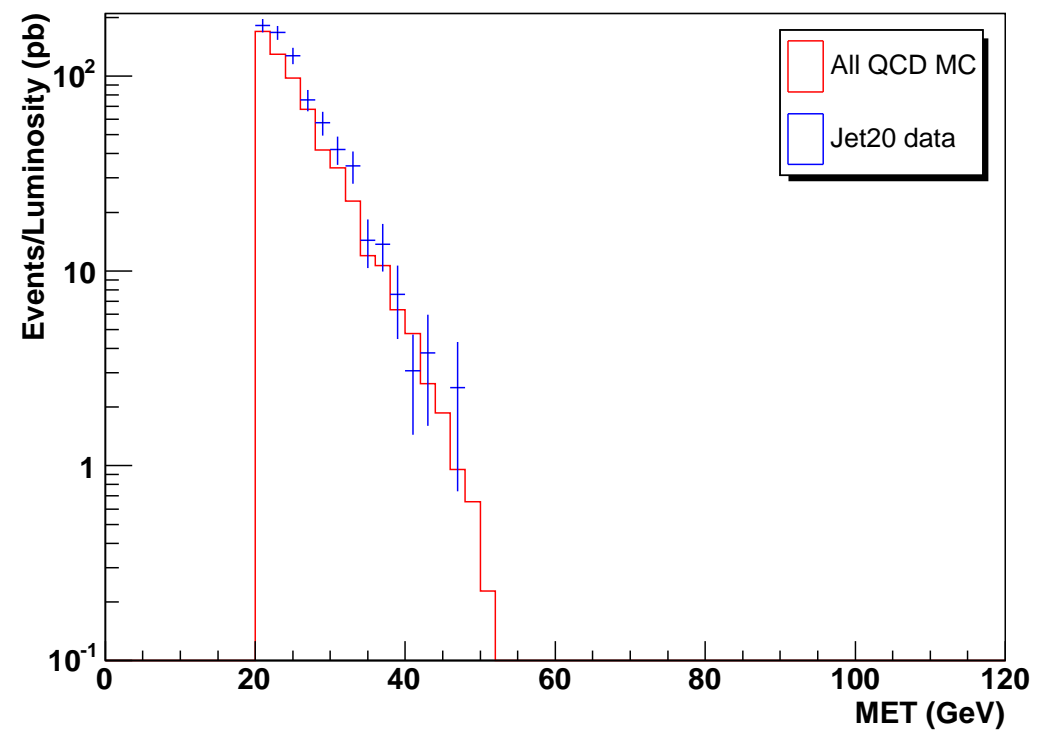

Fig. F.9: Distribution of $\not_{T}$ inside the QCD control region (zone 2). 
MET (zone 3: MET<20 and HT>=150)

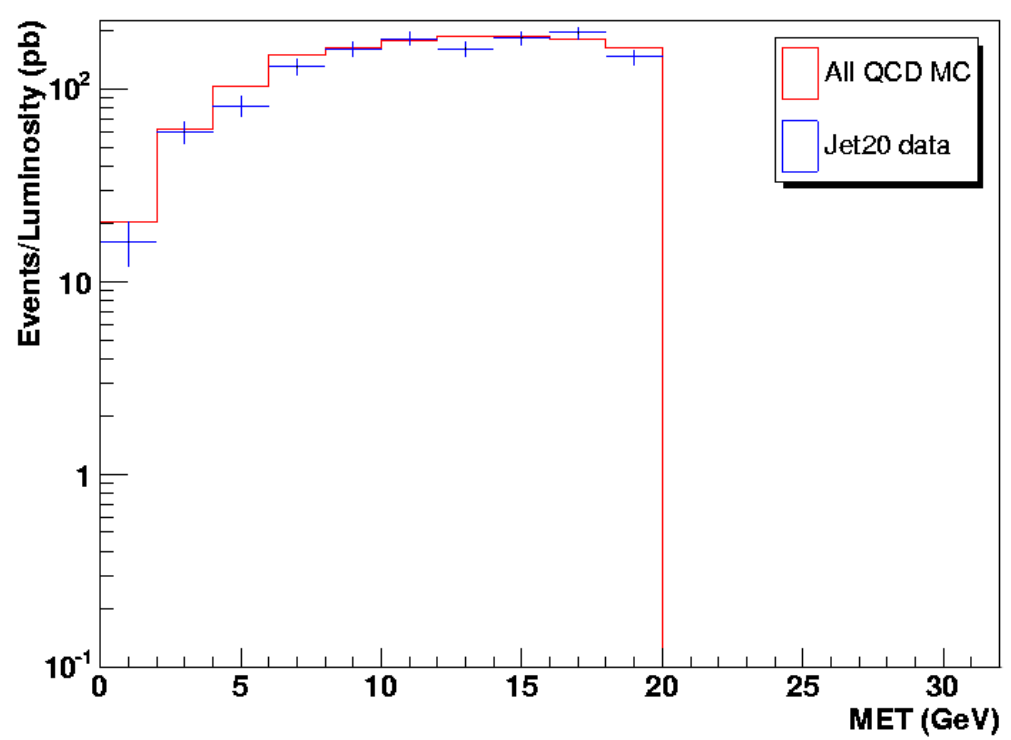

Fig. F.10: Distribution of $\not_{T}$ inside the QCD control region (zone 3).

\section{MET (zone 4: MET $>=20$ and $H T>=150$ )}

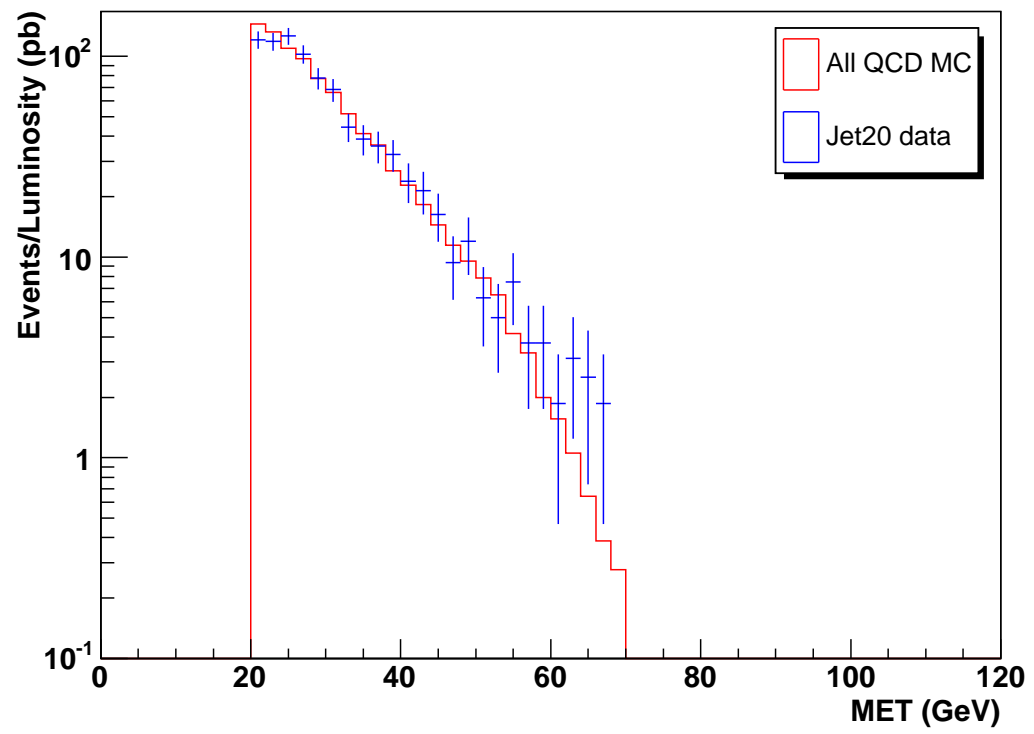

Fig. F.11: Distribution of $\not_{T}$ inside the QCD control region (zone 4). 


\section{$\hat{\mathbf{p}}_{\mathrm{T}}$ Study}

Since QCD cross sections vary dramatically depending on the transverse momentum involved in the process, the generation is performed in different $\hat{\mathrm{p}}_{\mathrm{T}}$ bins $^{1}$. Thus, it is important to determine the minimum $\hat{\mathrm{p}}_{\mathrm{T}}$ that contributes to the $\mathbb{E}_{T}$ trigger data, since it is virtually impossible to generate sufficient Monte Carlo statistics for an arbitrarily low $\hat{\mathrm{p}}_{\mathrm{T}}$ threshold. Hence, a minimum $\hat{\mathrm{p}}_{\mathrm{T}}$ threshold has been established by comparing the $\mathrm{MC}$ and data distributions as a function of decreasing $\hat{\mathrm{p}}_{\mathrm{T}}$ thresholds. Events are required to pass all the pre-selection cuts described in Section 4.1.2. The events that pass those cuts are completely dominated by the QCD multijet background.

Fig. F.12 shows Monte Carlo distributions of $\mathrm{H}_{\mathrm{T}}$, defined as $\mathrm{H}_{\mathrm{T}}=\mathrm{E}_{\mathrm{T}}^{\mathrm{jet} 1}+\mathrm{E}_{\mathrm{T}}^{\mathrm{jet}}+\mathrm{E}_{\mathrm{T}}^{\mathrm{jet} 3}$, for different $\hat{p}_{\mathrm{T}}$ cuts: $60,70,80,90,95,100,110,120$, and $130 \mathrm{GeV}$, compared to the data. Fig. F.14 shows similar plots for $\mathrm{E}_{\mathrm{T}}^{\mathrm{j} t 2}$. The ratios data/MC are shown in Fig. F.13 and Fig. F.15, respectively. From these plots one can see that the data favours a minimum $\hat{\mathrm{p}}_{\mathrm{T}}$ cut.
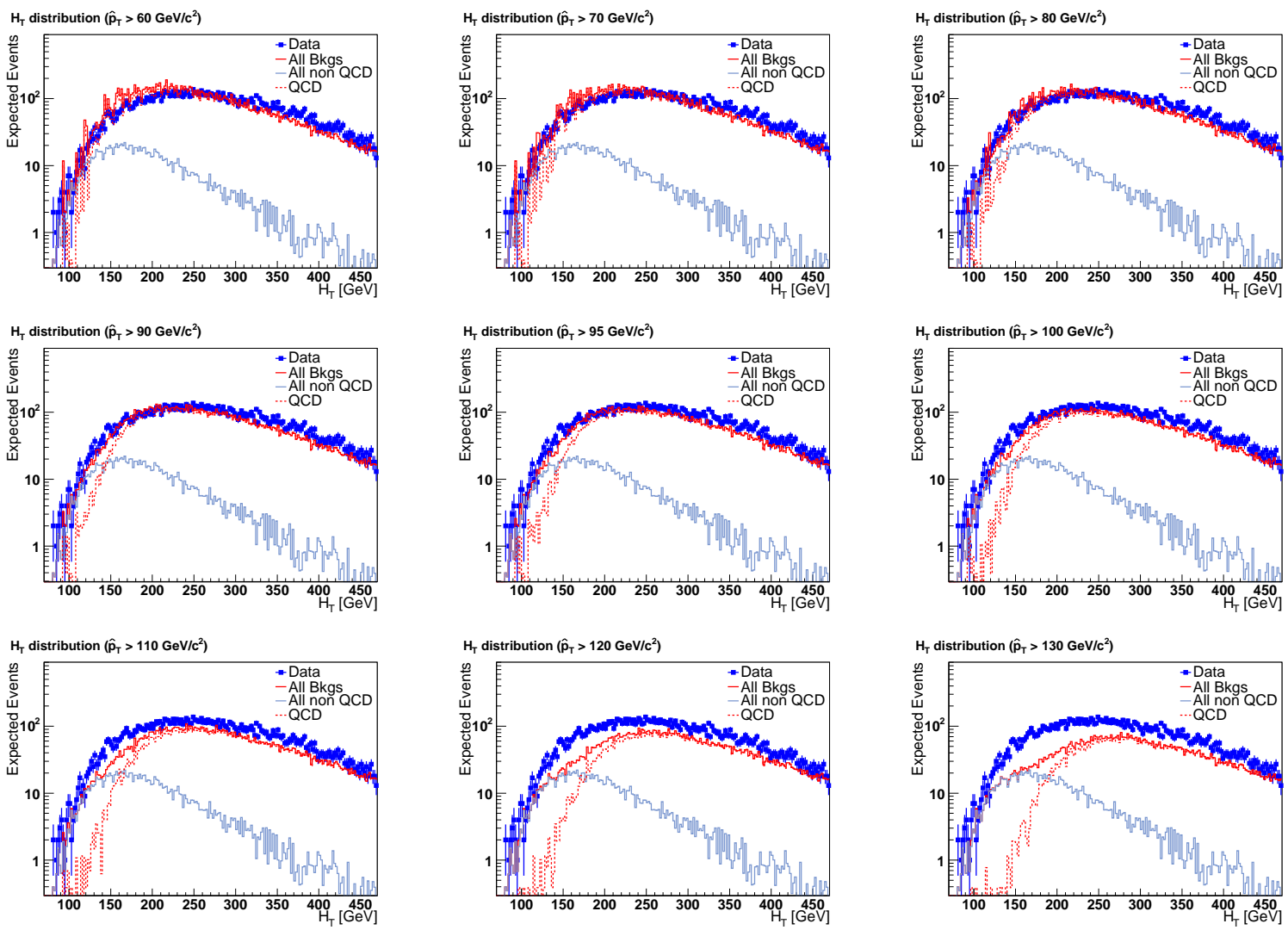

Fig. F.12: $\mathrm{H}_{\mathrm{T}}$ distributions for different minimum $\hat{\mathrm{p}}_{\mathrm{T}}$ cuts.

Thus, a $\chi^{2}$ fit is performed comparing the nine $\mathrm{H}_{\mathrm{T}}$ distributions to the data. The results are

\footnotetext{
${ }^{1}$ The $\hat{\mathrm{p}}_{\mathrm{T}}$ is the transverse momentum of the outgoing parton from the 2-to-2 process computed as in the center-ofmass.
} 

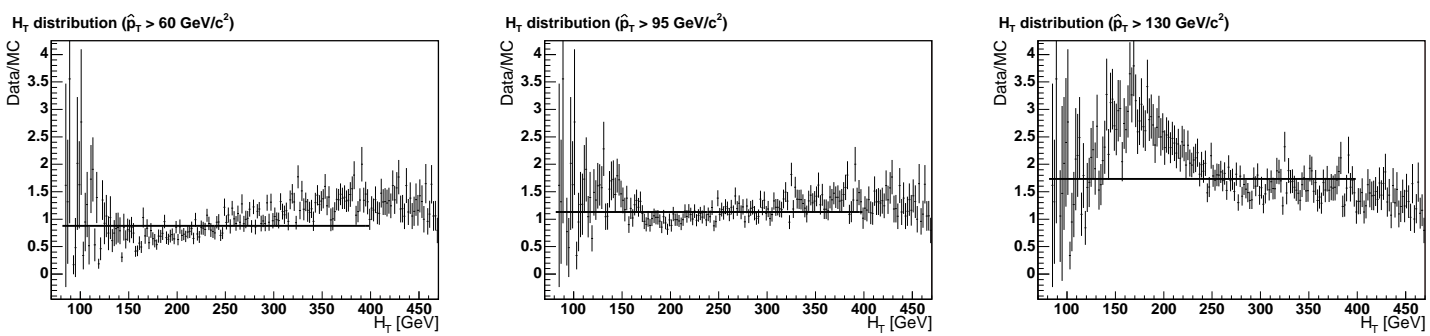

Fig. F.13: Ratio between the $\mathrm{H}_{\mathrm{T}}$ distributions of data and $\mathrm{MC}$ for three different $\hat{\mathrm{p}}_{\mathrm{T}}$ cuts: 60 , 95, and $130 \mathrm{GeV}$ respectively. The black line corresponds to the fit of the distribution.
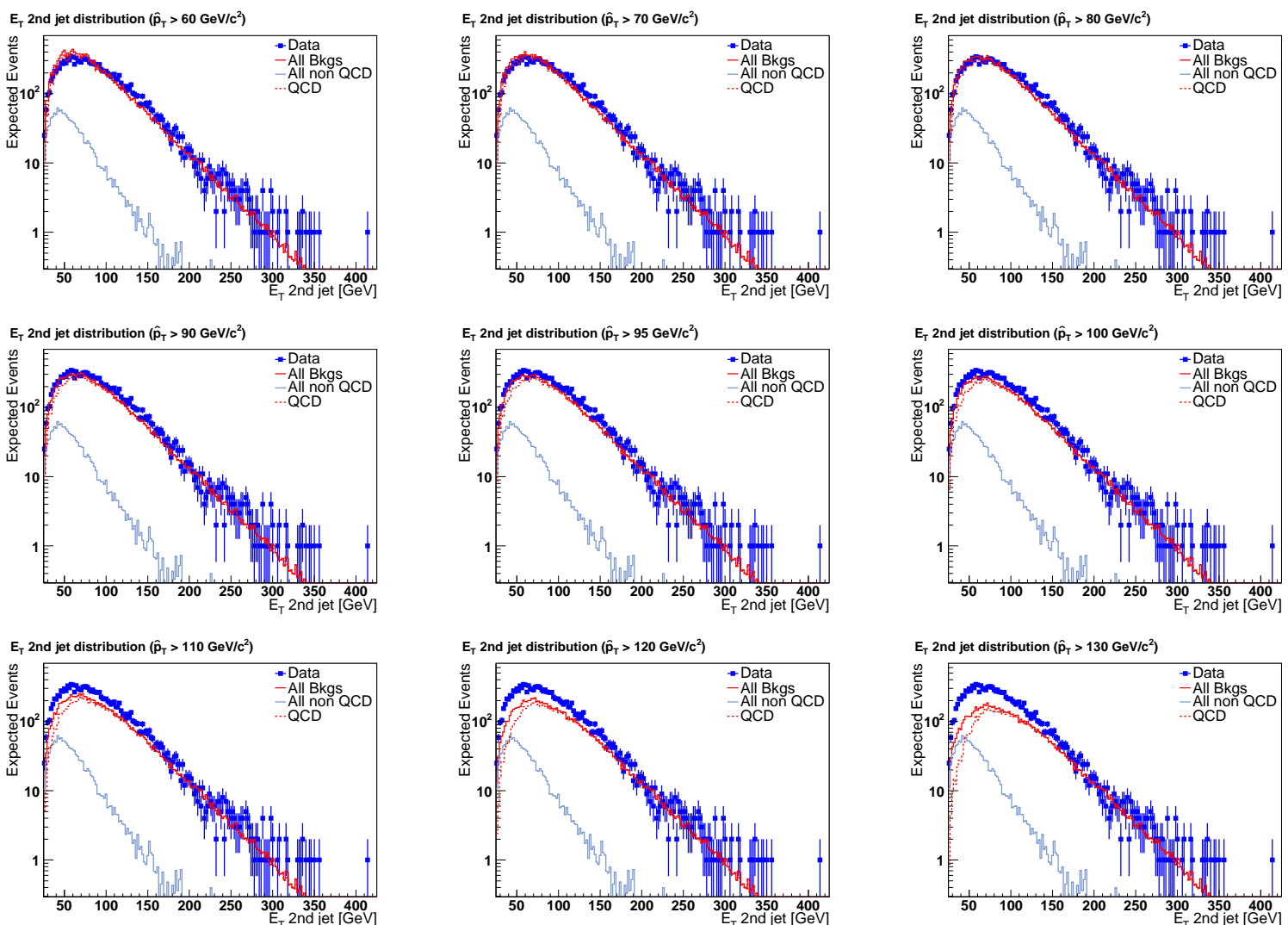

Fig. F.14: $\mathrm{E}_{\mathrm{T}}$ distribution of the second leading jet for different minimum $\hat{\mathrm{p}}_{\mathrm{T}}$ cuts.

shown in Fig. F.16. A similar study is done for the $\mathrm{E}_{\mathrm{T}}$ of the second leading jet distributions, Fig. F.17. Both fits suggest that the data is "best" reproduced with a $\hat{p}_{\mathrm{T}}$ around 95-100 GeV. Therefore, only QCD samples with $\hat{\mathrm{p}}_{\mathrm{T}} \gtrsim 90 \mathrm{GeV}$ are considered in the analysis. A significant effort was made to generate multijet QCD samples with an integrated luminosity significantly larger than the data. 

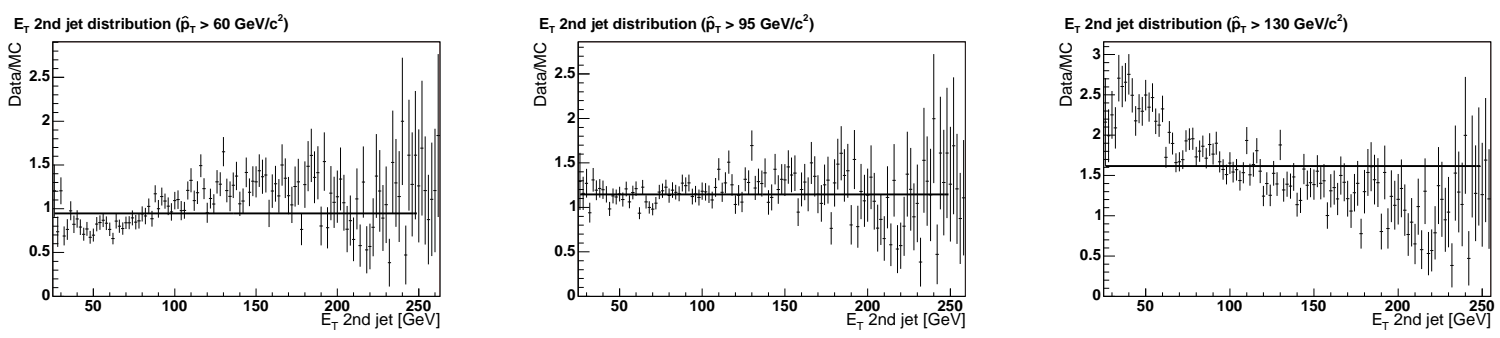

Fig. F.15: Ratio between the second leading jet's $\mathrm{E}_{\mathrm{T}}$ distributions of data and MC for three different $\hat{\mathrm{p}}_{\mathrm{T}}$ cuts: 60,95 , and $130 \mathrm{GeV}$, respectively. The black line corresponds to the fit of the distribution. 
$H_{T}$ distribution: $\chi^{2} /$ ndof for different $\hat{p}_{\mathrm{T}}^{\min }$ cuts

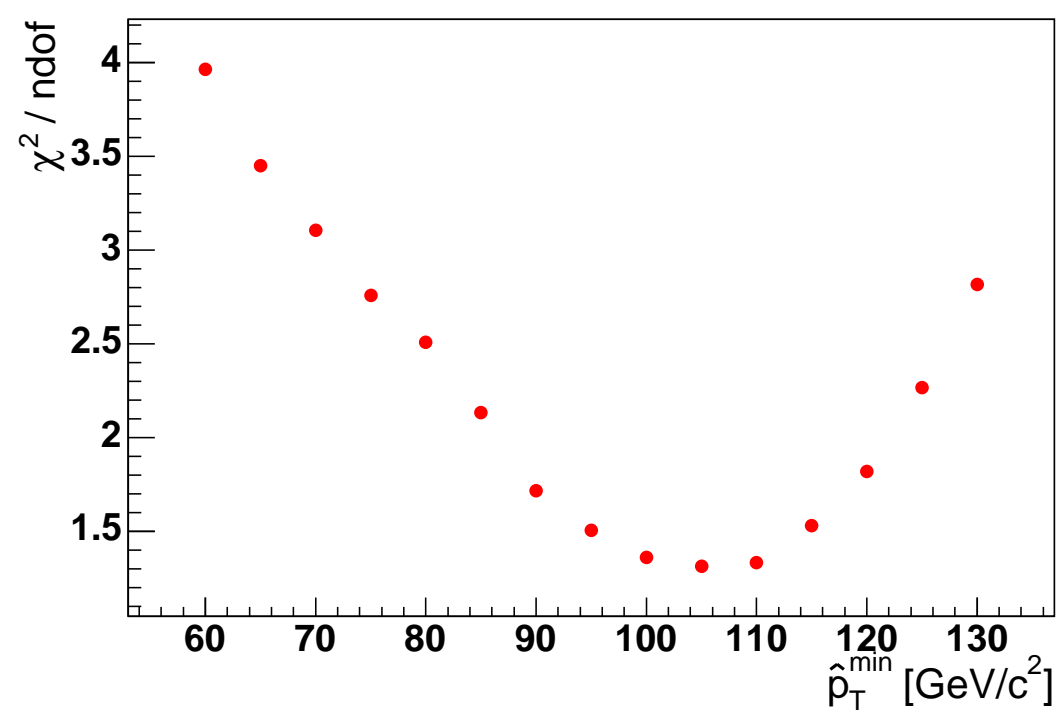

Fig. F.16: $\chi^{2}$ dd.o.f results from fitting the $\mathrm{H}_{\mathrm{T}}$ distributions to the data. The results suggest a minimum $\hat{\mathrm{p}}_{\mathrm{T}}$ cut around $95 \mathrm{GeV}$.

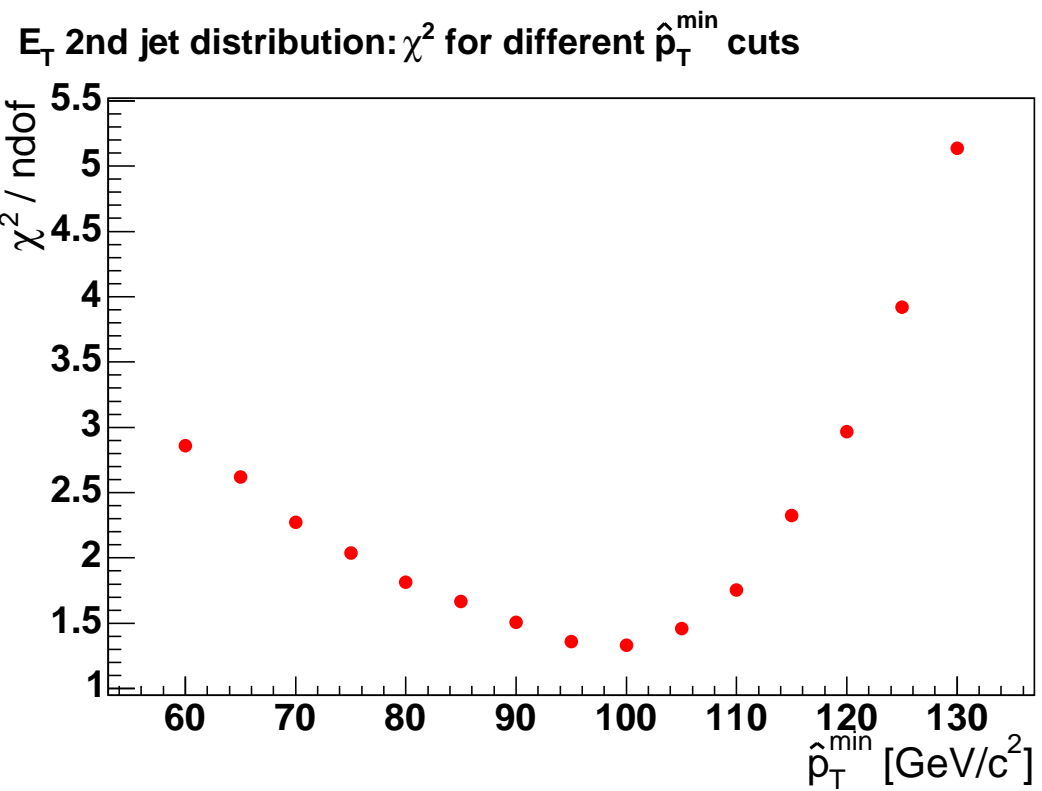

Fig. F.17: $\chi^{2} /$ d.o.f results from fitting the $\mathrm{E}_{\mathrm{T}}^{2}$ distributions to the data. The results above suggest a minimum $\hat{\mathrm{p}}_{\mathrm{T}}$ cut around $100 \mathrm{GeV}$. This is consistent with the $\mathrm{H}_{\mathrm{T}}$ results shown in Fig. F.16 


\section{Appendix G}

\section{Data vs MC Studies}

As discussed in Appendix F, a good agreement is observed between QCD multijet data and the Monte Carlo predictions. Therefore, it was concluded that no additional $k$-factor is needed to normalise the Monte Carlo predictions to the data. In that study, QCD multijet events were selected applying a necessary cut on the $\mathbb{E}_{T}$ significance that removed beam-related backgrounds in the data, and allowed a clean and well defined comparison with the Monte Carlo. As a consequence, most of QCD events with significant $\mathbb{E}_{T}$ were removed. However, the QCD events that constitute background to the mSUGRA analysis are characterised by the presence of large $\mathbb{E}_{T}$. A significant contribution comes from events with jets going into calorimeter cracks or close to the chimney region, where only a fraction of the jet energy is reconstructed.

Different quantities were compared between data and Monte Carlo to test the validity of the Monte Carlo description of the data for events with large $\mathbb{E}_{T}$ after the pre-selection cuts as well as the electron and muon removal cuts were applied (see Fig. G.1-G.3). The $\Delta \phi$ distribution between the $\mathbb{E}_{T}$ and each of the three leading jets in the event indicates, as expected, a dominant component for which the $\mathbb{E}_{T}$ is produced along the direction of the jets. A number of additional studies were carried out to further understand the origin of the small discrepancies shown in the distributions. Detailed comparisons were performed between data and Monte Carlo for jets reconstructed around the cracks, and the chimney regions. From them, it was concluded that the observed differences could be partially attributed to small defects on the simulation of the calorimeter response. After a cut on $\Delta \phi\left(j e t, E_{\mathrm{T}}\right)>0.7$ is applied (see Fig. G.4 through Fig. G.6), most of the QCD multijet background is removed and the discrepancies are within systematics.

The rather conservative systematic uncertainties on the QCD Monte Carlo normalisation account for the remaining differences between data and Monte Carlo which, in addition to the energy scale, includes a $20 \%$ uncertainty from PDFs and a $10 \%$ uncertainty from hard scale dependencies. Fig. G.7 shows the $\mathbb{E}_{T}$ and the $\mathrm{H}_{\mathrm{T}}$ distributions after the pre-selection cuts. Fig. G.8 shows the $\mathbb{E}_{T}$ and $\mathrm{H}_{\mathrm{T}}$ distributions with all the systematics and after all other cuts are applied. 
The agreement between data and MC is good in all the zones.

In addition, the $\Delta \phi\left(j e t, E_{T}\right)$ and the EMF requirements were reversed to enhance the QCD and boson+jets electromagnetic backgrounds separately, and test that the Monte Carlo properly describes each different background contribution (see Fig. G.9 to Fig. G.10). In addition, the muon contribution has been studied reversing the muon cuts (asking for at least one isolated track) as shown in Fig. G.11. All the distributions show a reasonable agreement between data and Monte Carlo. 

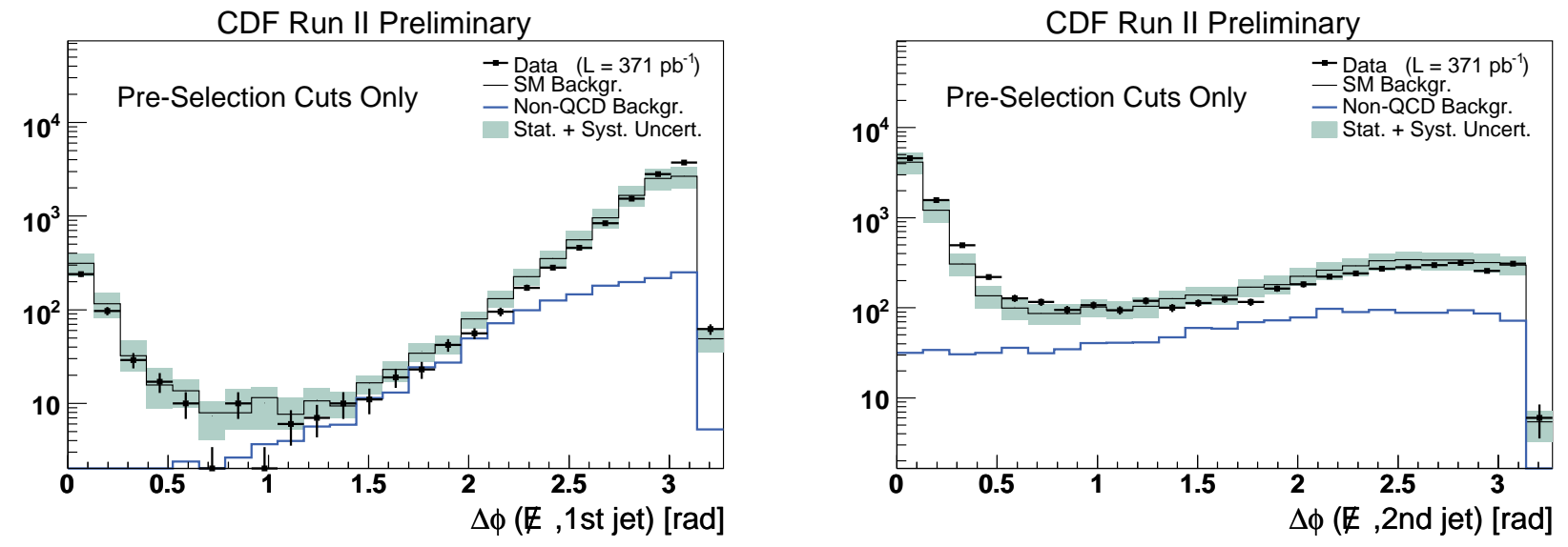

CDF Run II Preliminary

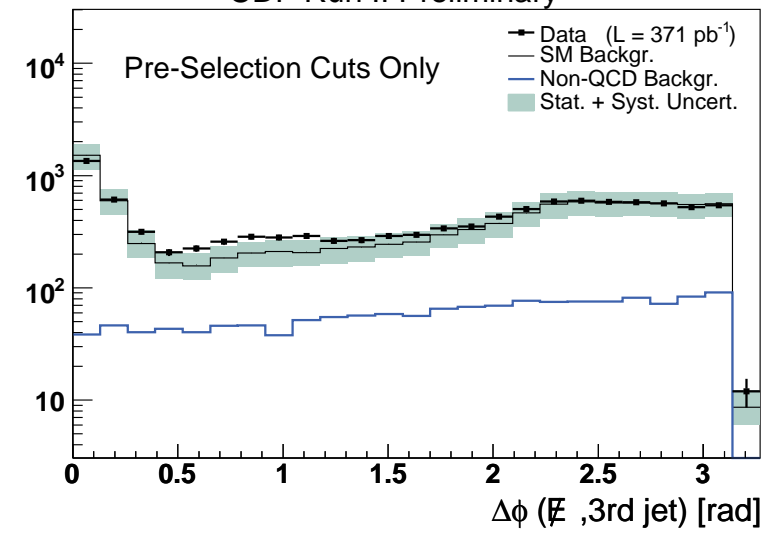

Fig. G.1: Azimuthal distance between $\not_{T}$ and $\mathrm{E}_{\mathrm{T}}$ of the three leading jets for data and $\mathrm{MC}$ after pre-selection, electron and muon removal cuts have been applied. 

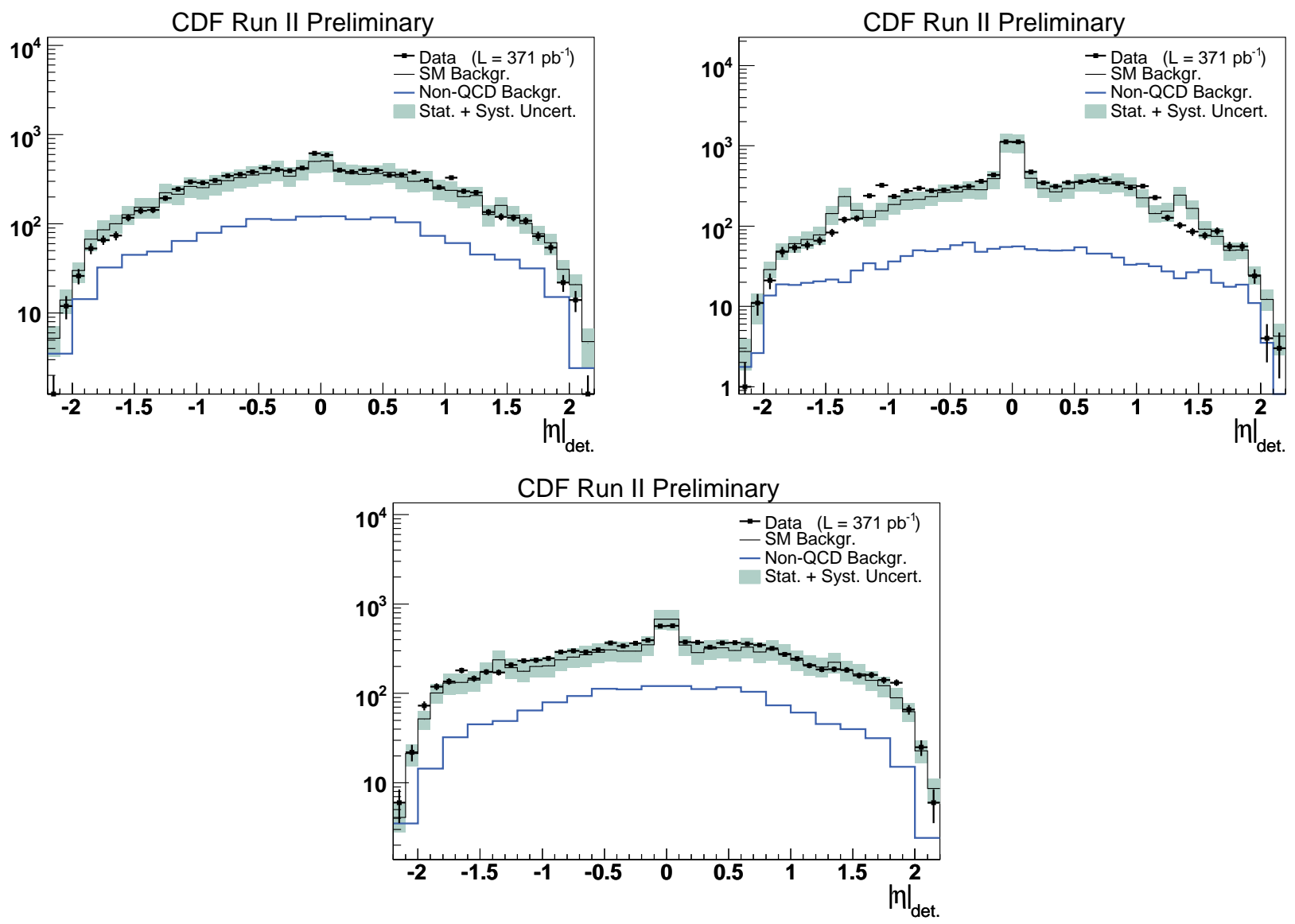

Fig. G.2: Detector $\eta$ of the three leading jets for data and MC after pre-selection, electron and muon removal cuts have been applied. 

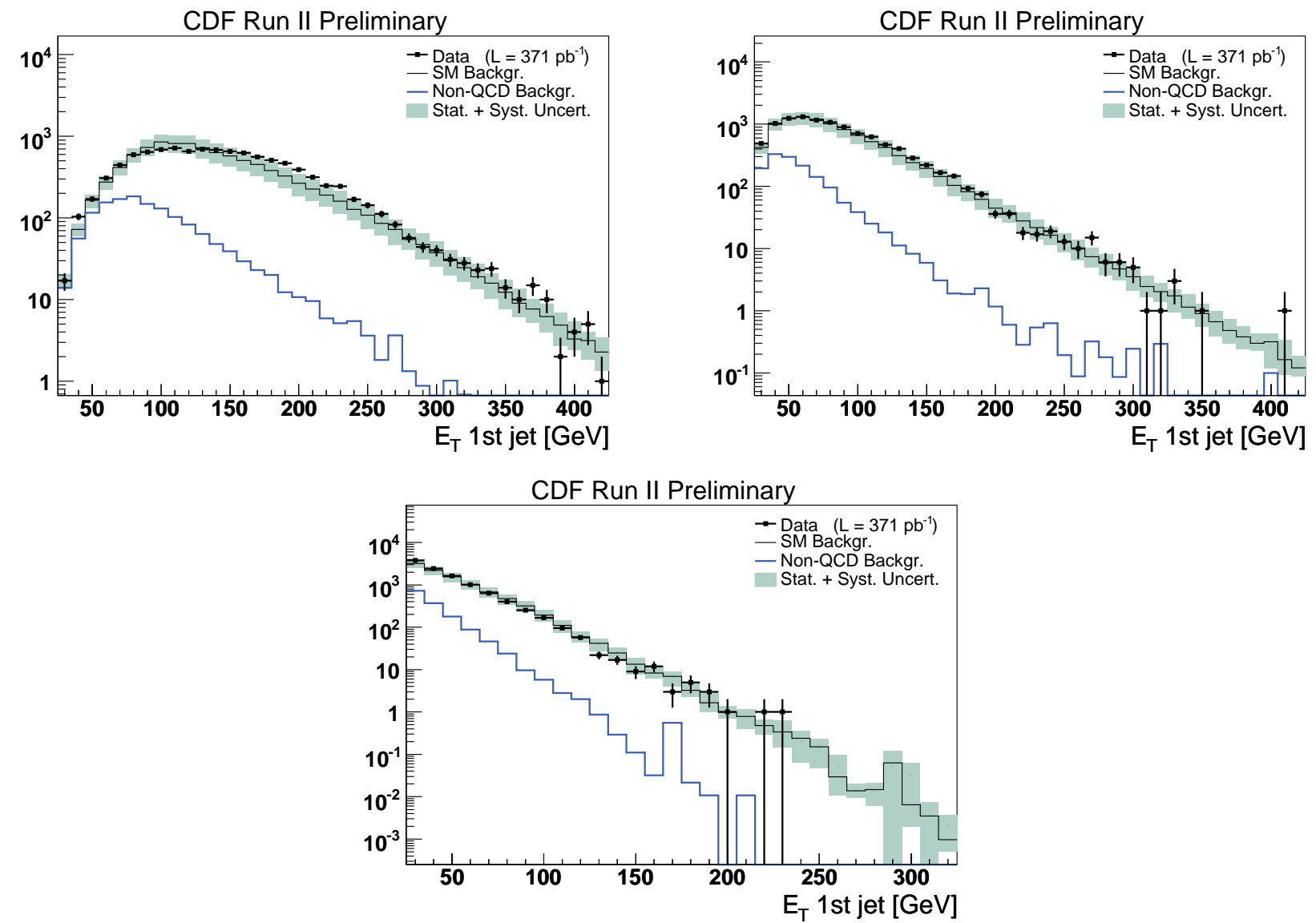

Fig. G.3: $\mathrm{E}_{\mathrm{T}}$ of the three leading jets for data and $\mathrm{MC}$ after pre-selection, electron and muon removal cuts have been applied. There are 10551 data events and $9.87_{-2.33}^{+1.35} \cdot 10^{3}$ Monte Carlo events in the plots. 

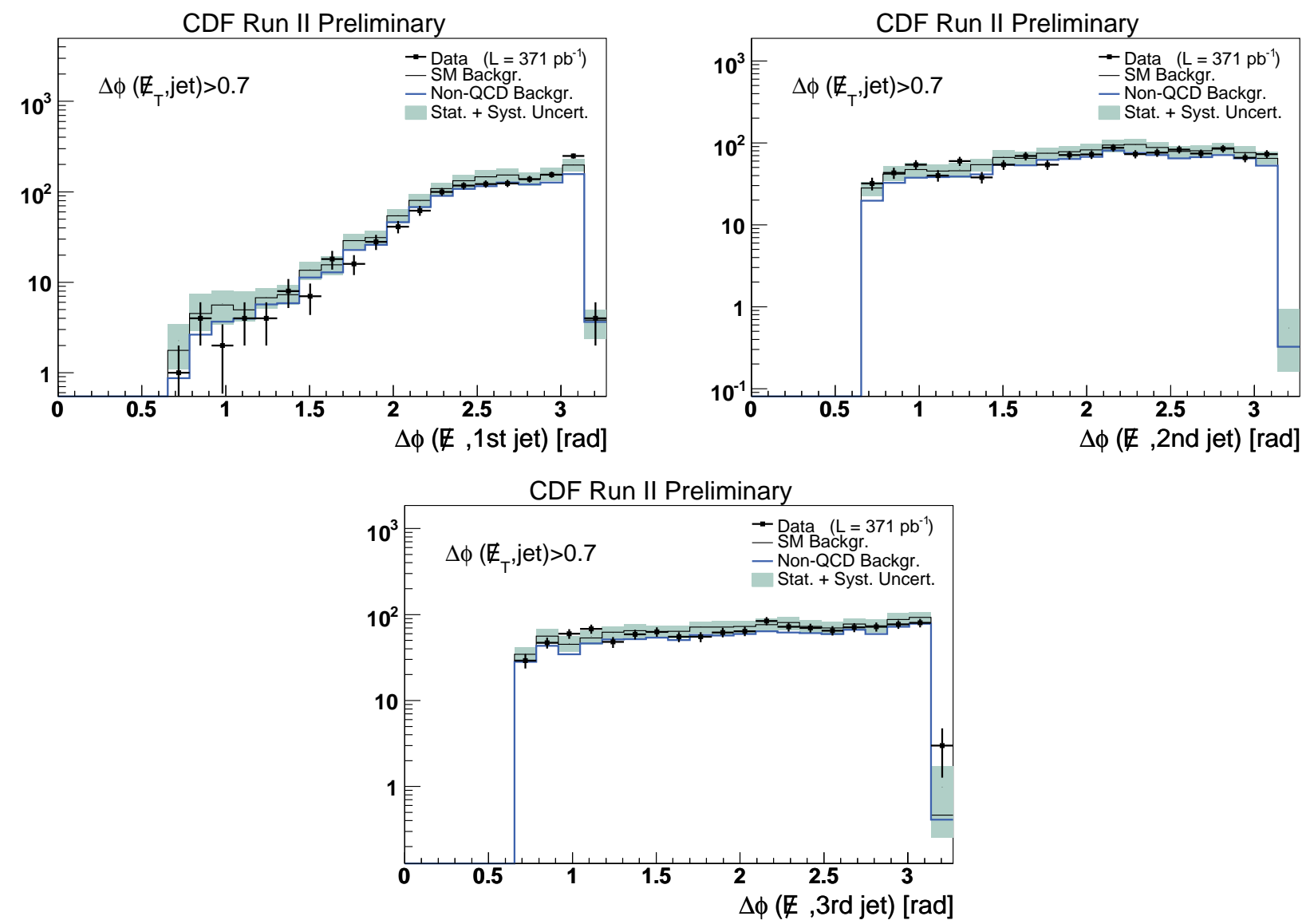

Fig. G.4: Azimuthal distance between $\mathbb{H}_{T}$ and $\mathrm{E}_{\mathrm{T}}$ of the three leading jets for data and MC after the $\Delta \phi\left(\mathbb{E}_{T}\right.$, jets $)>$ 0.7 cuts. Pre-selection, electron and muon removals cuts have also been applied. There are 1204 data events and $1.29_{-0.17}^{+0.14} \cdot 10^{3} \mathrm{MC}$ events in the plots. 

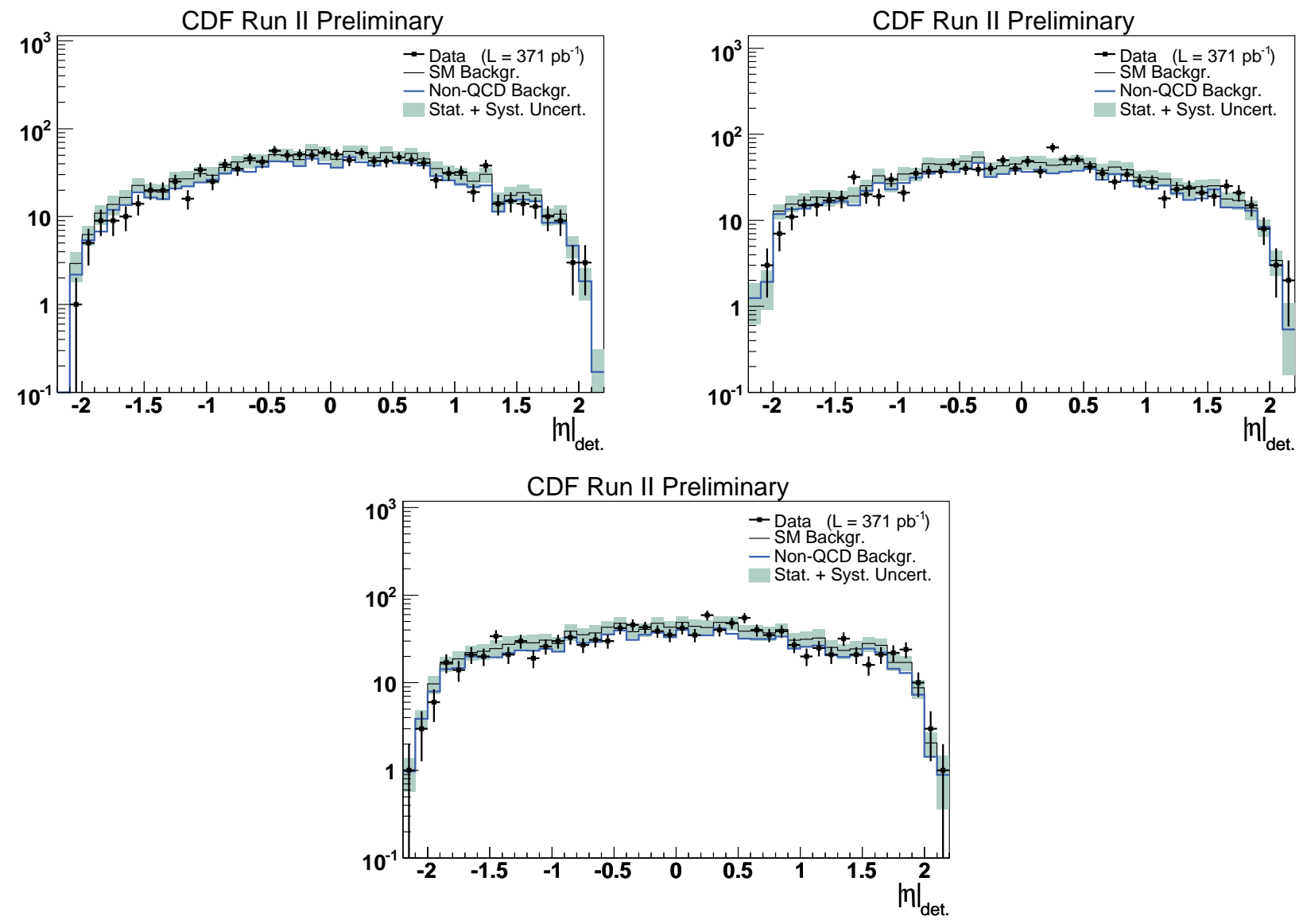

Fig. G.5: Detector $\eta$ of the three leading jets for data and MC after the $\Delta \phi\left(\mathbb{E}_{T}\right.$, jets $)>0.7$ cuts. Pre-selection, electron and muon removals cuts have also been applied. 
CDF Run II Preliminary

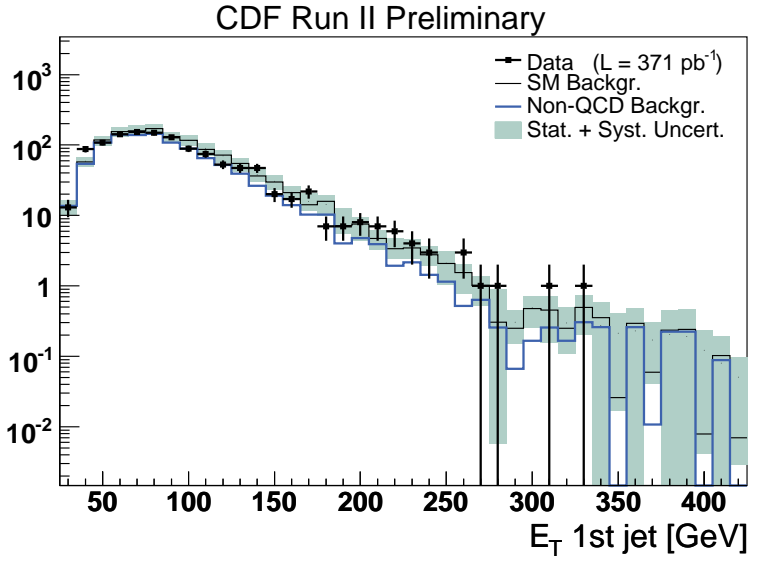

CDF Run II Preliminary

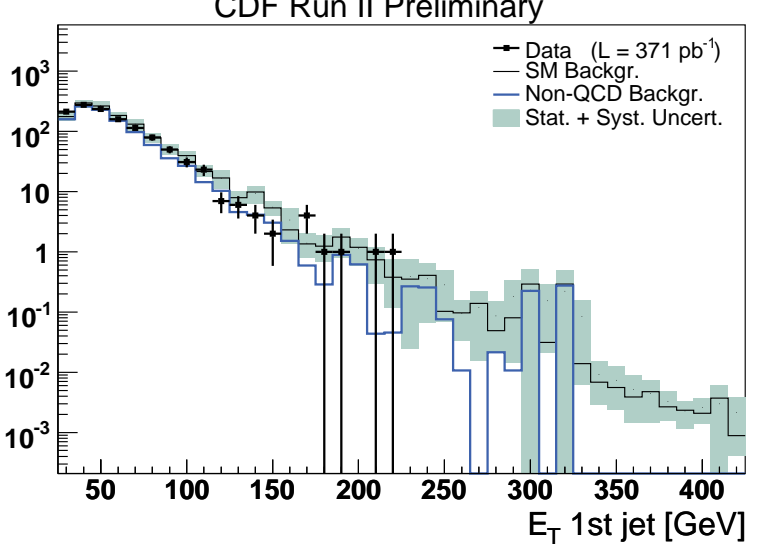

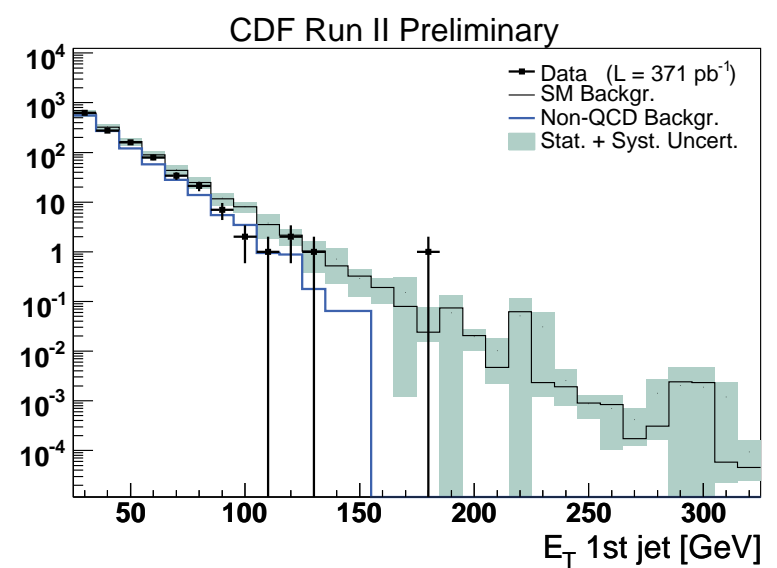

Fig. G.6: $\mathrm{E}_{\mathrm{T}}$ of the three leading jets for data and $\mathrm{MC}$ after the $\Delta \phi\left(\mathbb{E}_{T}\right.$,jets $)>0.7$ cuts. Pre-selection, electron and muon removals cuts have also been applied. 

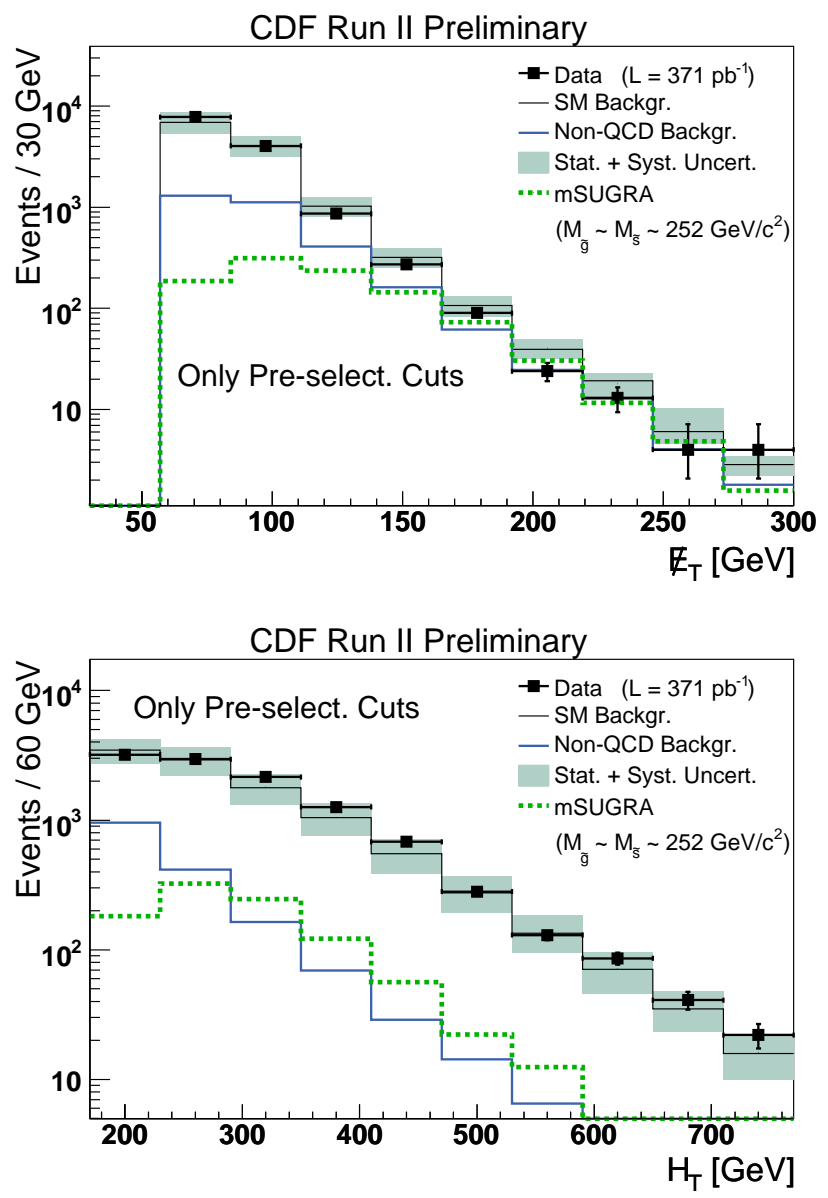

Fig. G.7: $\not_{T}$ and $\mathrm{H}_{\mathrm{T}}$ just after the pre-selection cuts. 

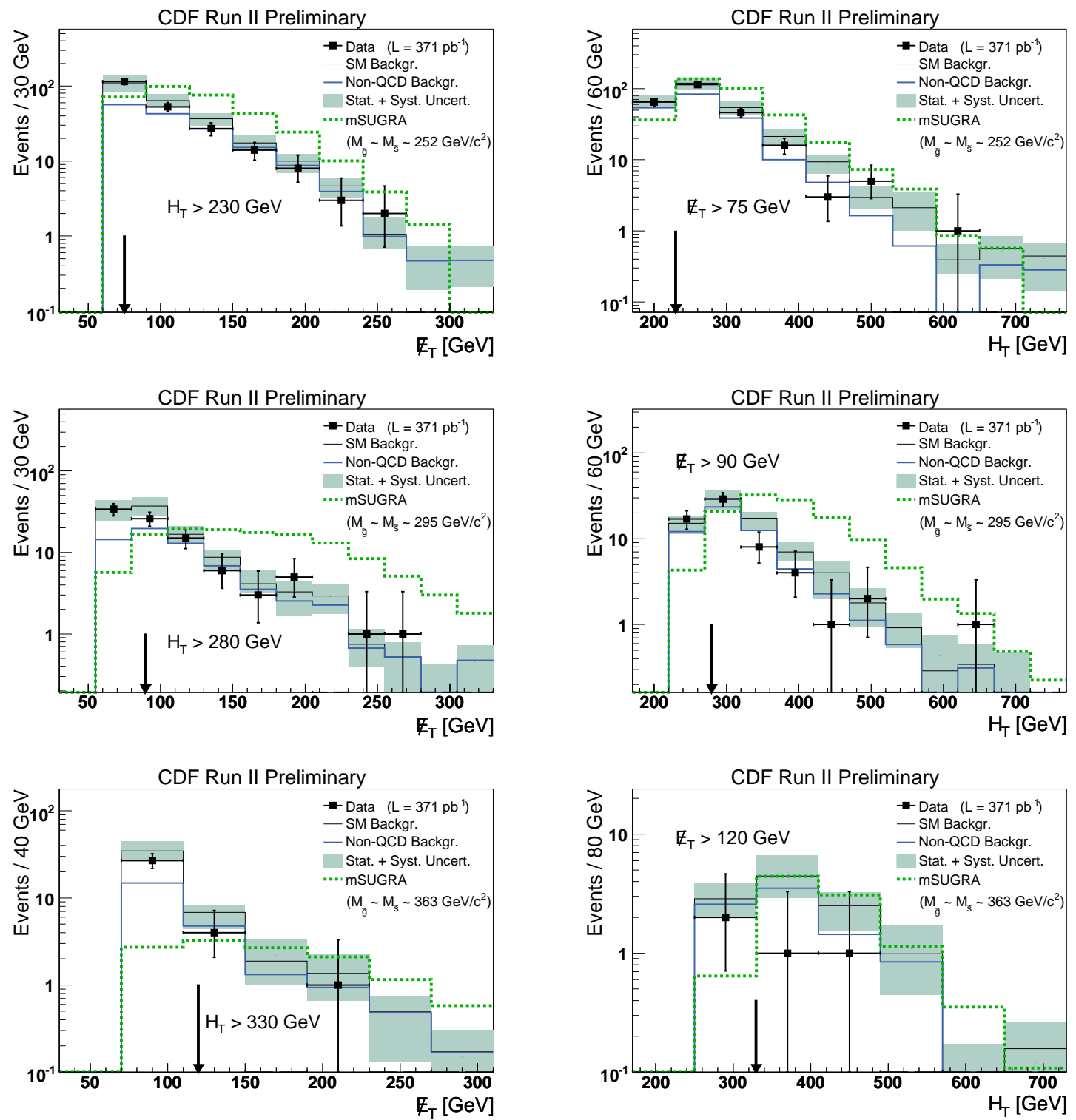

Fig. G.8: $\not_{T}$ and $\mathrm{H}_{\mathrm{T}}$ after all cuts in the analysis except the $\not_{T}$ and $\mathrm{H}_{\mathrm{T}}$ cuts respectively, but reversing the $\Delta \phi\left(\not_{T}\right.$, jets $)>0.7$ cut by asking at least one of the three jets to fail the condition. All three zones are shown. 

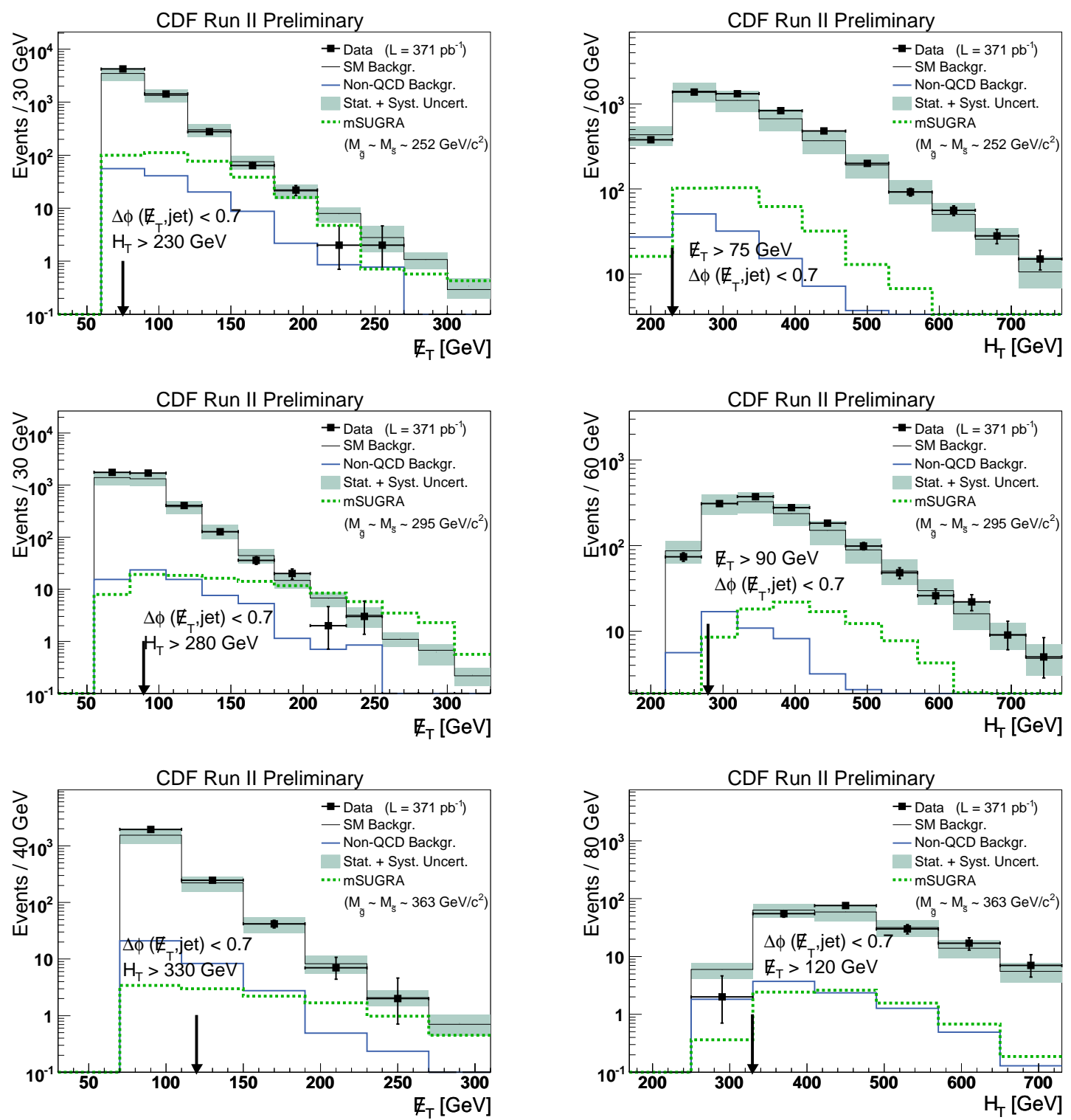

Fig. G.9: $\not_{T}$ and $\mathrm{H}_{\mathrm{T}}$ after all cuts in the analysis except the $\not_{T}$ and $\mathrm{H}_{\mathrm{T}}$ cuts respectively, but reversing the $\Delta \phi\left(\not_{T}\right.$, jets $)>0.7$ cut by asking at least one of the three jets to fail the condition. All three zones are shown. 

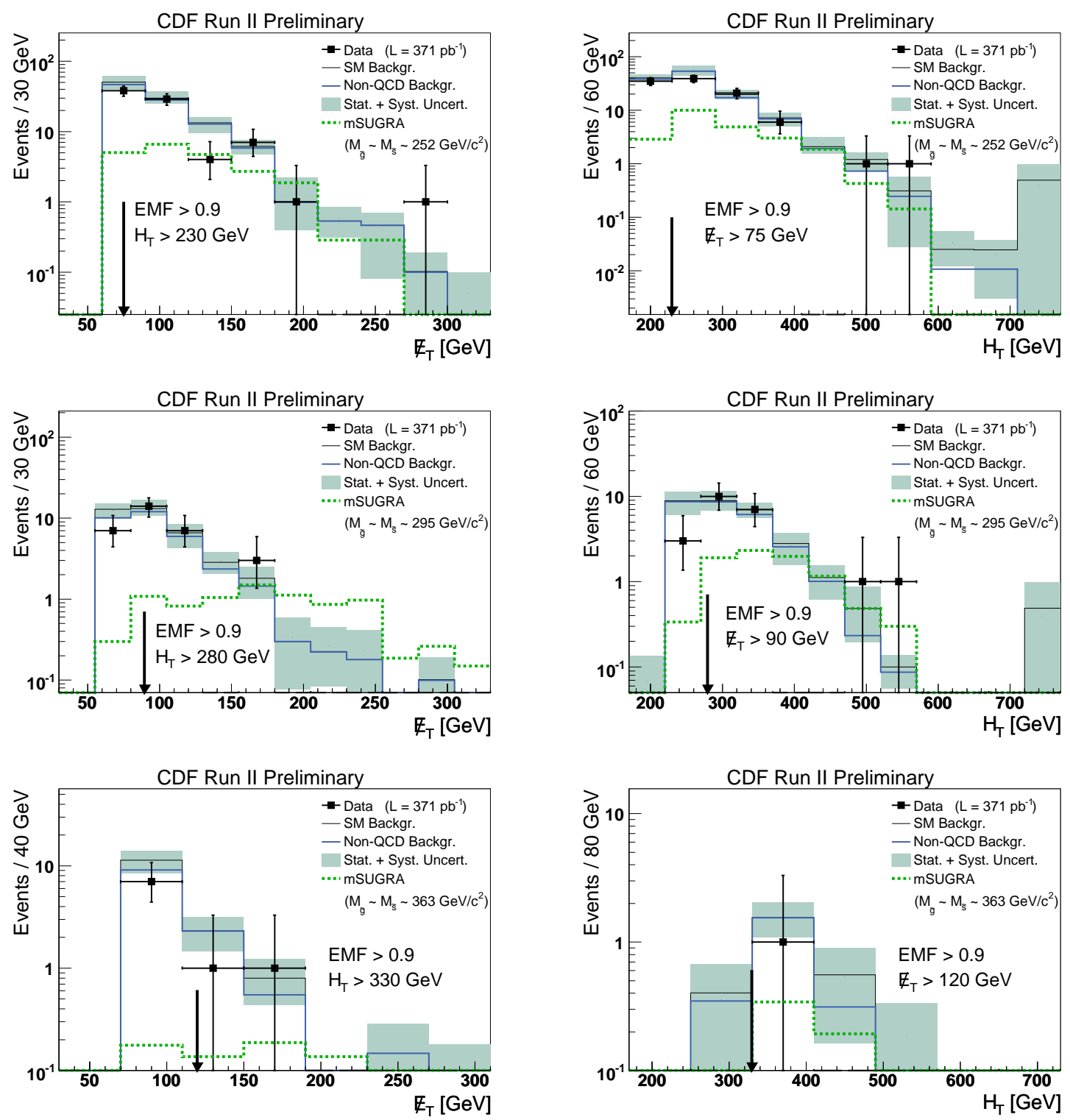

Fig. G.10: $\not_{T}$ and $\mathrm{H}_{\mathrm{T}}$ after all cuts in the analysis except the $\mathscr{E}_{T}$ and $\mathrm{H}_{\mathrm{T}}$ cuts respectively, but reversing the EMF $>0.9$ cuts by asking at least one of the three jets to fail the condition. All three zones are shown. 

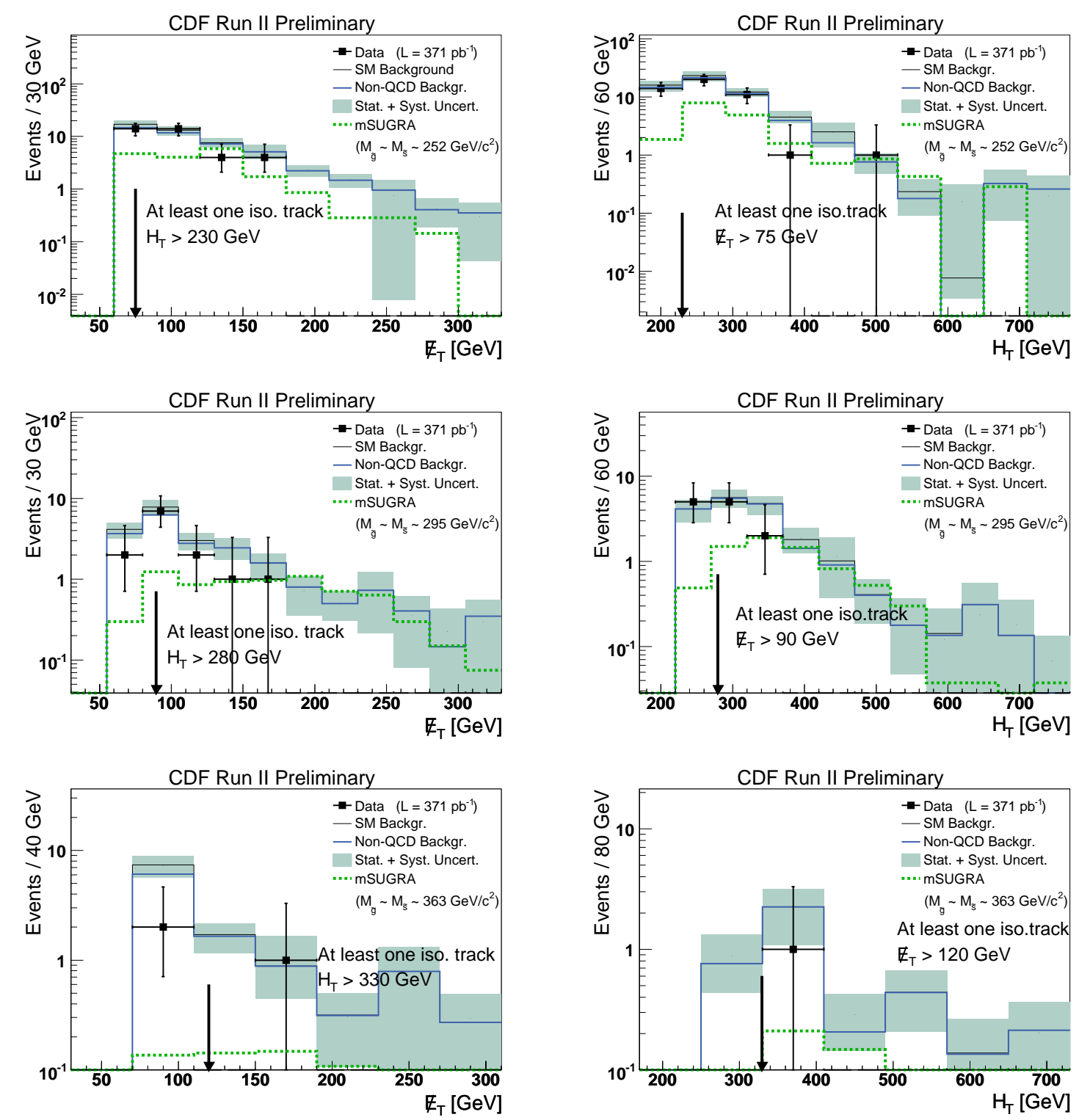

Fig. G.11: $\not_{T}$ and $\mathrm{H}_{\mathrm{T}}$ after all cuts in the analysis except the $\not_{T}$ and $\mathrm{H}_{\mathrm{T}}$ cuts respectively, but enhancing the muon contribution by requiring at least one isolated track. All three zones are shown. 